\title{
Effect of Substituents on Radical Stability in Reversible Addition Fragmentation Chain Transfer (RAFT) Polymerization: An Ab Initio Study
}

\author{
Michelle L. Coote ${ }^{*}$ and David J. Henry ${ }^{\dagger}$ \\ Research School of Chemistry, Australian National University, Canberra, ACT 0200, Australia
}

SUPPORTING INFORMATION

(Tables S1-S4: 44 pages total)

\footnotetext{
*To whom correspondence should be addressed, email: mcoote@rsc.anu.edu.au

${ }^{\dagger}$ Current Address: Department Of Applied Physics, RMIT University, GPO Box 2476V, Melbourne VIC 3001, Australia
} 
Table S1. Geometries of the RAFT-adduct Radicals, and Corresponding Alkanes

and Thiocarbonyl Products of Beta-Scission, Used in Calculating the Radical

Stabilization Energies and Beta-Scission Enthalpies in Table 1

\begin{abstract}
$\mathrm{CH}_{3} \mathrm{SC} \cdot(\mathrm{H}) \mathrm{S}-\mathrm{CH}_{3}(1)$

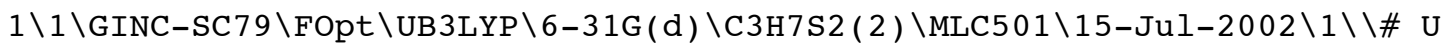
B3LYP $/ 6-31 \mathrm{G} *$ FOPT $=(\mathrm{Z}-\mathrm{MATRIX}, \mathrm{MAXCYC}=100)$ FREQ MAXDISK $=131072000 \mathrm{SCF}=(\mathrm{TI}$

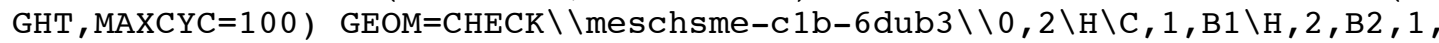
$\mathrm{A} 1 \backslash \mathrm{S}, 2, \mathrm{~B} 3,1, \mathrm{~A} 2,3, \mathrm{D} 1,0 \backslash \mathrm{C}, 4, \mathrm{~B} 4,2, \mathrm{~A} 3,1, \mathrm{D} 2,0 \backslash \mathrm{S}, 5, \mathrm{~B} 5,4, \mathrm{~A} 4,2, \mathrm{D} 3,0 \backslash \mathrm{C}, 6, \mathrm{~B} 6,5, \mathrm{~A}$ $5,4,-\mathrm{D} 4,0 \backslash \mathrm{H}, 7, \mathrm{~B} 7,6, \mathrm{~A} 6,5, \mathrm{D} 5,0 \backslash \mathrm{H}, 7, \mathrm{~B} 8,6, \mathrm{~A} 7,8, \mathrm{D} 6,0 \backslash \mathrm{H}, 7, \mathrm{~B} 9,6, \mathrm{~A} 8,8, \mathrm{D} 7,0 \backslash \mathrm{H}, 5$ , $\mathrm{B} 10,4, \mathrm{~A} 9,2, \mathrm{D} 8,0 \backslash \mathrm{H}, 2, \mathrm{~B} 11,1, \mathrm{~A} 10,3, \mathrm{D} 9,0 \backslash \backslash \mathrm{B} 1=1.09245285 \backslash \mathrm{B} 2=1.0938637 \backslash \mathrm{B} 3=1$ $.83125984 \backslash \mathrm{B} 4=1.75500785 \backslash \mathrm{B} 5=1.74076721 \backslash \mathrm{B} 6=1.83589742 \backslash \mathrm{B} 7=1.09250381 \backslash \mathrm{B} 8=1$ $.09126763 \backslash \mathrm{B} 9=1.09314848 \backslash \mathrm{B} 10=1.08750952 \backslash \mathrm{B} 11=1.09312093 \backslash \mathrm{A} 1=109.89583381 \backslash$ $\mathrm{A} 2=110.82435935 \backslash \mathrm{A} 3=100.23333582 \backslash \mathrm{A} 4=122.50571955 \backslash \mathrm{A} 5=103.40041602 \backslash \mathrm{A} 6=110$ $.32952264 \backslash \mathrm{A} 7=111.31841879 \backslash \mathrm{A} 8=106.03792498 \backslash \mathrm{A} 9=118.05982812 \backslash \mathrm{A} 10=109.5874$ $6805 \backslash D 1=-122.6210925 \backslash D 2=64.69226164 \backslash D 3=-158.0946175 \backslash D 4=-51.30757768 \backslash D 5$ $=59.46321196 \backslash D 6=-122.46199608 \backslash D 7=118.68665702 \backslash D 8=47.35009589 \backslash D 9=119.69$ $751863 \backslash \backslash$ Version=DEC-AXP-OSF $/ 1-G 98 \mathrm{RevA} .11 .3 \backslash \mathrm{HF}=-914.8590203 \backslash \mathrm{S} 2=0.755128$ $\backslash \mathrm{S} 2-1=0 . \backslash \mathrm{S} 2 \mathrm{~A}=0.75002 \backslash \mathrm{RMSD}=5.785 \mathrm{e}-09 \backslash \mathrm{RMSF}=1.037 \mathrm{e}-05 \backslash \mathrm{Dipole}=0.0181274,0$. $5574054,-0.4697362 \backslash P G=C 01 \quad[X(C 3 H 7 S 2)] \backslash \backslash @$
\end{abstract}

\title{
$\mathrm{CH}_{3} \mathrm{SCH}(\mathrm{H}) \mathrm{S}-\mathrm{CH}_{3}$
}

$1 \backslash 1 \backslash G I N C-S C 6 \backslash F O p t \backslash R B 3 L Y P \backslash 6-31 G(d) \backslash C 3 H 8 S 2 \backslash M L C 501 \backslash 16-J a n-2003 \backslash 0 \backslash \backslash \# N$ B 3LY

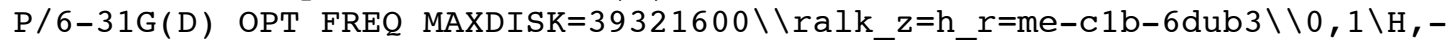
$1.0151419089,0.6632849312,2.6972163132 \backslash \mathrm{C}, 0.078 \overline{3} 529214,0.6950522212,2.6$ $558703823 \backslash \mathrm{H}, 0.4183652806,1.734664888,2.6882136211 \backslash \mathrm{S}, 0.7159739462,-0.17$ $47976705,1.1782594292 \backslash \mathrm{C},-0.0947554845,0.8318071219,-0.1346138624 \backslash \mathrm{S}, 0.0$ $310859694,0.0645597733,-1.7811338337 \backslash \mathrm{C},-1.1437776621,-1.3236098164,-1$. $5943840921 \backslash \mathrm{H},-2.1616942804,-0.958564836,-1.4265197574 \backslash \mathrm{H},-0.8430792875$, $-1.9813714536,-0.775789725 \backslash \mathrm{H},-1.1177694117,-1.8828323647,-2.5332204445$ $\backslash \mathrm{H}, 0.3960859392,1.8055913112,-0.2208422596 \backslash \mathrm{H}, 0.4773571681,0.1697755482$

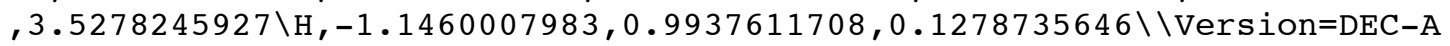
$\mathrm{XP}-\mathrm{OSF} / 1-\mathrm{G} 98 \mathrm{ReVA} .11 .3 \backslash \mathrm{HF}=-915.509719 \backslash \mathrm{RMSD}=7.837 \mathrm{e}-09 \backslash \mathrm{RMSF}=9.091 \mathrm{e}-06 \backslash \mathrm{Dip}$ ole $=-0.7853892,0.2460225,0.5234409 \backslash \mathrm{PG}=\mathrm{C} 01$ [X(C3H8S2) ] \\}

\section{$\mathrm{S}=\mathrm{C}(\mathrm{H}) \mathrm{SCH}_{3}$}

$1 \backslash 1 \backslash G I N C-S C 28 \backslash F O p t \backslash R B 3 L Y P \backslash 6-31 G(d) \backslash C 2 H 4 S 2 \backslash M L C 501 \backslash 16-J u 1-2002 \backslash 1 \backslash \backslash \# P$ RB3 LYP /6-31G * FOPT $=($ TIGHT, MAXCYC $=150$, Z-MATRIX $)$ MAXDISK=131072000 FREQ SCF $=\mathrm{TIGHT} \backslash \backslash \mathrm{S}=\mathrm{C}(\mathrm{H})-\mathrm{S}-\mathrm{Me} \mathrm{Cs}(2 \mathrm{a}) \backslash \backslash 0,1 \backslash \mathrm{S} \backslash \mathrm{C}, 1, \mathrm{~b} 1 \backslash \mathrm{H}, 2, \mathrm{~b} 2,1, \mathrm{a} 1 \backslash \mathrm{S}, 2, \mathrm{~b} 3,1, \mathrm{a} 2,3,18$ 0 . $0 \backslash \mathrm{C}, 4, \mathrm{~b} 4,2, \mathrm{a} 3,1,0 ., 0 \backslash \mathrm{H}, 5, \mathrm{~b} 5,4, \mathrm{a} 4,2,180.0 \backslash \mathrm{H}, 5, \mathrm{~b} 6,4, \mathrm{a} 5,2, \mathrm{dh} 1,0 \backslash \mathrm{H}, 5, \mathrm{~b}$ $6,4, a 5,2,-d h 1,0 \backslash \backslash \mathrm{b} 1=1.63746361 \backslash \mathrm{b} 2=1.09330009 \backslash \mathrm{b} 3=1.73755944 \backslash \mathrm{b} 4=1.820696$ $57 \backslash \mathrm{b} 5=1.09233155 \backslash \mathrm{b} 6=1.09203481 \backslash \mathrm{a} 1=121.28809715 \backslash \mathrm{a} 2=129.63000423 \backslash \mathrm{a} 3=103$. $10279807 \backslash \mathrm{a} 4=106.35470404 \backslash \mathrm{a} 5=110.18674431 \backslash \mathrm{dh} 1=60.3841272 \backslash \backslash$ Version=DEC $-\mathrm{A}$ $\mathrm{XP}-\mathrm{OSF} / 1-\mathrm{G} 98 \mathrm{RevA} .11 .3 \backslash \mathrm{State}=1-\mathrm{A}^{\prime} \backslash \mathrm{HF}=-874.9871381 \backslash \mathrm{RMSD}=5.554 \mathrm{e}-09 \backslash \mathrm{RMSF}=5$ $.472 \mathrm{e}-06 \backslash \mathrm{Dipole}=-0.6513287,0 ., 0.6109301 \backslash \mathrm{PG}=\mathrm{CS}[\mathrm{SG}(\mathrm{C} 2 \mathrm{H} 2 \mathrm{~S} 2), \mathrm{X}(\mathrm{H} 2)] \backslash \backslash @$

\section{$\mathrm{CH}_{3} \mathrm{SC} \cdot(\mathrm{Cl}) \mathrm{SCH}_{3}(2)$}

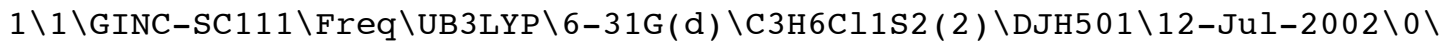
$\backslash \# N$ UB3LYP/6-31G(D) SCF=TIGHT FREQ MAXDISK=39321600 \\CH3SCClSCH3 radic al UB3LYP / 6-31G(d) freq $\backslash \backslash 0,2 \backslash \mathrm{Cl}, 0 ., 0 ., 1.7458857534 \backslash \mathrm{C}, 0 \ldots, 0 \ldots,-0.015644$ $2466 \backslash S, 0.730536,1.315209,-0.8890652466 \backslash S,-0.730536,-1.315209,-0.889065$ $2466 \backslash \mathrm{C}, 0 ., 2.808624,-0.1091052466 \backslash \mathrm{C}, 0 .,-2.808624,-0.1091052466 \backslash \mathrm{H},-1.080$ $263,2.837085,-0.2685442466 \backslash \mathrm{H}, 0.227201,2.837952,0.9583197534 \backslash \mathrm{H}, 0.468179$ $, 3.664216,-0.6031962466 \backslash \mathrm{H}, 1.080263,-2.837085,-0.2685442466 \backslash \mathrm{H},-0.227201$ 
$,-2.837952,0.9583197534 \backslash \mathrm{H},-0.468179,-3.664216,-0.6031962466 \backslash \backslash$ Version=D EC-AXP-OSF /1-G98RevA.11.3 \State $=2-B \backslash H F=-1374.454289 \backslash \mathrm{S} 2=0.755828 \backslash \mathrm{S} 2-1=0$ $. \backslash \mathrm{S} 2 \mathrm{~A}=0.750025 \backslash \mathrm{RMSD}=5.641 \mathrm{e}-09 \backslash \mathrm{RMSF}=1.672 \mathrm{e}-06 \backslash \mathrm{Dipole}=0 ., 0 ., 0.1376501 \backslash$ $\mathrm{PG}=\mathrm{C} 02[\mathrm{C} 2(\mathrm{C} 1 \mathrm{Cl} 1), \mathrm{X}(\mathrm{C} 2 \mathrm{H} 6 \mathrm{~S} 2)] \backslash \backslash @$

\section{$\mathrm{CH}_{3} \mathrm{SCH}(\mathrm{Cl}) \mathrm{SCH}_{3}$}

$1 \backslash 1 \backslash G I N C-S C 111 \backslash$ Freq $\backslash R B 3 L Y P \backslash 6-31 G(d) \backslash C 3 H 7 C 11 S 2 \backslash D J H 501 \backslash 11-A u g-2003 \backslash 0 \backslash \backslash \# N$ B3LYP/6-31G(D) SCF=TIGHT FREQ MAXDISK=39321600\\CH3SCHClSCH3e B3LYP / $6-31 \mathrm{G}(\mathrm{d})$ freq $\backslash \backslash 0,1 \backslash \mathrm{Cl}, 0.2146509865,1.8154969189,0.1747001216 \backslash \mathrm{C}, 0.0300$ $199865,0.1401209189,-0.6036308784 \backslash S,-1.6969700135,-0.3982130811,-0.709$ $0948784 \backslash \mathrm{C},-2.1683300135,-0.5234140811,1.0531761216 \backslash \mathrm{S}, 1.0768459865,-1.1$ $193530811,0.1923921216 \backslash \mathrm{C}, 2.7333879865,-0.3688570811,0.0148271216 \backslash \mathrm{H}, 2.7$ $950059865,0.5734479189,0.5630811216 \backslash \mathrm{H}, 2.9869469865,-0.2104800811,-1.03$ $74008784 \backslash \mathrm{H}, 0.3470769865,0.2972469189,-1.6354788784 \backslash \mathrm{H},-2.0072210135,0.4$ $329109189,1.5558411216 \backslash \mathrm{H},-1.6206740135,-1.3178680811,1.5630501216 \backslash \mathrm{H},-3$ $.2358420135,-0.7582880811,1.0568011216 \backslash \mathrm{H}, 3.4371569865,-1.0864570811,0$. $4452141216 \backslash \backslash$ Version=DEC-AXP-OSF / 1-G03RevB.02 $\backslash$ State $=1-\mathrm{A} \backslash \mathrm{HF}=-1375.101057$ $\backslash \mathrm{RMSD}=5.230 \mathrm{e}-09 \backslash \mathrm{RMSF}=2.090 \mathrm{e}-06 \backslash \mathrm{Dipole}=0.3716004,-0.2438015,0.1086554$ $\backslash \mathrm{PG}=\mathrm{C} 01[\mathrm{X}(\mathrm{C} 3 \mathrm{H} 7 \mathrm{Cl} 1 \mathrm{~S} 2)] \backslash \backslash @$

\section{$\mathrm{CH}_{3} \mathrm{~S}(\mathrm{CI}) \mathrm{C}=\mathrm{S}$}

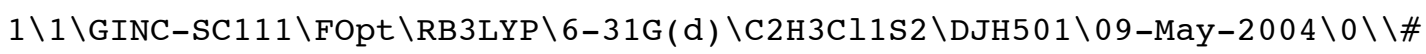
B3LYP/6-31G* SCF=TIGHT OPT=TIGHT MAXDISK=39321600 \CH3SCCl=Sa B3LYP / $6-31 \mathrm{G}(\mathrm{d})$ geom opt $\backslash \backslash 0,1 \backslash \mathrm{Cl}, 0.033089777,0,-2.0304760456 \backslash \mathrm{C},-0.2403988803$ , 0 . $,-0.2710280846 \backslash \mathrm{S}, 1.3166697464,0 \ldots, 0.5239390117 \backslash \mathrm{S},-1.7280471353,0$. 0 . $3846713207 \backslash \mathrm{C}, 0.8528974077,0$. $2.2853874397 \backslash \mathrm{H}, 1.7963221294,0$. 2.83822126 $17 \backslash \mathrm{H}, 0.2740993601,0.8932837385,2.527975033 \backslash \mathrm{H}, 0.2740993601,-0.893283738$ $5,2.527975033 \backslash \backslash$ Version=DEC-AXP-OSF $/ 1-G 03 R e v B$. 05 $\backslash$ State $=1-A^{\prime} \backslash \mathrm{HF}=-1334.58$ $1472 \backslash \mathrm{RMSD}=2.831 \mathrm{e}-09 \backslash \mathrm{RMSF}=1.027 \mathrm{e}-06 \backslash \mathrm{Dipole}=0.6442117,0.0 .9198297 \backslash \mathrm{PG}=\mathrm{CS}$ [SG(C2H1Cl1S2), X(H2)]\\@

\section{$\mathrm{CH}_{3} \mathrm{SC} \cdot(\mathrm{C} \equiv \mathrm{CH}) \mathrm{SCH}_{3}(3)$}

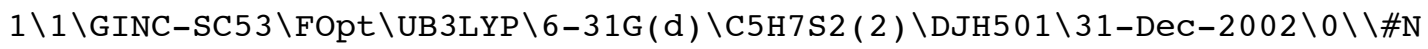
UB3LYP/6-31G(D) SCF=TIGHT OPT=TIGHT MAXDISK=39321600 \CH3SC (CCH) SCH $3 \mathrm{a}$ radical UB3LYP / 6-31G(d) geom opt \\0,2\C,-1.3935126015,-0.0001480506, $-0.0025533332 \backslash \mathrm{C},-0.0124262998,0.0000084787,-0.0000218284 \backslash \mathrm{S}, 0.951953598$ $, 1.3900139503,0.5075682919 \backslash \mathrm{S}, 0.9540939966,-1.3898131262,-0.5040928334 \backslash$ $\mathrm{C},-0.0852911905,2.7966454975,-0.0343717844 \backslash \mathrm{C},-0.084834507,-2.796652869$ $4,0.034091939 \backslash \mathrm{H}, 0.4238359495,3.7002415898,0.3109459011 \backslash \mathrm{H},-1.0776965565$ $, 2.7418481291,0.4178252589 \backslash \mathrm{H},-0.1712141752,2.816908105,-1.1233128227 \backslash \mathrm{H}$ $, 0.4257492741,-3.7001503781,-0.3093261506 \backslash \mathrm{H},-0.1747431267,-2.816891924$ $3,1.1227092969 \backslash \mathrm{H},-1.0755829579,-2.7420739854,-0.421751501 \backslash \mathrm{C},-2.6178320$ $265,-0.0002952656,-0.0048046979 \backslash \mathrm{H},-3.6837301689,-0.0004414652,-0.00673$ $90891 \backslash \backslash$ Version=DEC-AXP-OSF $/ 1-G 98 \mathrm{RevA} .11 .3 \backslash \mathrm{HF}=-991.0155763 \backslash \mathrm{S} 2=0.76416 \backslash \mathrm{S}$ $2-1=0 . \backslash \mathrm{S} 2 \mathrm{~A}=0.750114 \backslash \mathrm{RMSD}=6.012 \mathrm{e}-09 \backslash \mathrm{RMSF}=1.215 \mathrm{e}-06 \backslash \mathrm{Dipole}=-0.7768784,-0$ $.0000695,-0.0013946 \backslash \mathrm{PG}=\mathrm{C} 01[\mathrm{X}(\mathrm{C} 5 \mathrm{H} 7 \mathrm{~S} 2)] \backslash \backslash @$

\section{$\mathrm{CH}_{3} \mathrm{SCH}(\mathrm{C} \equiv \mathrm{CH}) \mathrm{SCH}_{3}$}

$1 \backslash 1 \backslash G I N C-S C 82 \backslash F O p t \backslash R B 3 L Y P \backslash 6-31 G(d) \backslash C 5 H 8 S 2 \backslash D J H 501 \backslash 27-A u g-2002 \backslash 0 \backslash \backslash \# N$ B3L YP/6-31G(D) SCF=TIGHT OPT=TIGHT MAXDISK=39321600\\CH3SCH(CCH) SCH3a B3L

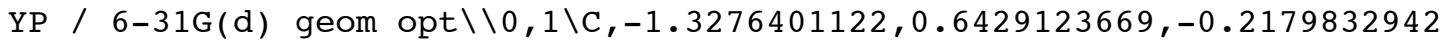
$\backslash \mathrm{C}, 0.1243882062,0.6426339763,-0.2352818921 \backslash \mathrm{H}, 0.4973029679,1.6642727503$ 
$,-0.3662994452 \backslash S, 0.8689781304,-0.0444251055,1.328668416 \backslash S, 0.8291057708$ $,-0.2583587998,-1.6851811168 \backslash \mathrm{C},-0.0338045377,0.9150394309,2.5975071558$ $\backslash \mathrm{C},-0.0374443498,-1.8628694219,-1.5687675453 \backslash \mathrm{H},-1.1114451894,0.7524948$ $512,2.5188272134 \backslash \mathrm{H}, 0.1892857992,1.9839097136,2.5269470219 \backslash \mathrm{C},-2.5360638$ $735,0.6330315005,-0.1860803034 \backslash \mathrm{H},-3.6025468583,0.6315354428,-0.1726020$ $704 \backslash \mathrm{H},-1.1192422261,-1.7209392298,-1.6264578589 \backslash \mathrm{H}, 0.2252651919,-2.3883$ $678612,-0.6482200924 \backslash \mathrm{H}, 0.2983907086,-2.4476309475,-2.4292328571 \backslash \mathrm{H}, 0.31$ $7035189,0.5447806497,3.5648765766 \backslash \backslash$ Version=DEC-AXP-OSF / 1-G98RevA.11.3\ $\mathrm{HF}=-991.6438868 \backslash \mathrm{RMSD}=5.051 \mathrm{e}-09 \backslash \mathrm{RMSF}=3.895 e-06 \backslash \mathrm{Dipole}=-0.8027486,0.0938$ $106,0.3219046 \backslash \mathrm{PG}=\mathrm{C} 01 \quad[\mathrm{X}(\mathrm{C} 5 \mathrm{H} 8 \mathrm{~S} 2)] \backslash \backslash @$

\section{$\mathrm{CH}_{3} \mathrm{~S}(\mathrm{C} \equiv \mathrm{CH}) \mathrm{C}=\mathrm{S}$}

$1 \backslash 1 \backslash G I N C-S C 118 \backslash F O p t \backslash R B 3 L Y P \backslash 6-31 G(d) \backslash C 4 H 4 S 2 \backslash D J H 501 \backslash 16-M a y-2004 \backslash 0 \backslash \backslash \#$ B3L YP/6-31G(D) SCF=TIGHT OPT=TIGHT MAXDISK=39321600 \CH3SC (CCH) $=\mathrm{Sa}$ B3LYP / 6-31G(d) geom opt $\backslash \backslash 0,1 \backslash \mathrm{C},-1.7584688591,-0.1451473439,0 . \backslash \mathrm{C},-0.3408545$ $857,-0.2491757558,0 . \backslash S, 0.414450599,1.3462403054,0$. \S, $0.4399086213,-1.7$ $073319807,0 . \backslash \mathrm{C}, 2.1888985838,0.9495391396,0 . \backslash \mathrm{C},-2.9652194701,-0.0407140$ $395,0 . \backslash \mathrm{H},-4.0300992955,0.0266060125,0 . \backslash \mathrm{H}, 2.708897524,1.9110863413,0 . \backslash \mathrm{H}$ $, 2.4526601162,0.3763812237,0.8913290945 \backslash \mathrm{H}, 2.4526601162,0.3763812237,-0$ $.8913290945 \backslash \backslash$ Version=DEC-AXP-OSF / 1-G03RevB.05 \State=1-A ' \HF=-951.12816 $33 \backslash \mathrm{RMSD}=3.560 \mathrm{e}-09 \backslash \mathrm{RMSF}=2.917 \mathrm{e}-06 \backslash \mathrm{Dipole}=0.0100958,0.7721737,0 . \backslash \mathrm{PG}=\mathrm{CS}$ [ $\mathrm{SG}(\mathrm{C} 4 \mathrm{H} 2 \mathrm{~S} 2), \mathrm{X}(\mathrm{H} 2)] \backslash \backslash$ @

\section{$\mathrm{CH}_{3} \mathrm{SC} \cdot\left(\mathrm{CH}=\mathrm{CH}_{2}\right) \mathrm{SCH}_{3}(4)$}

$1 \backslash 1 \backslash$ GINC-SC14 $\backslash$ Freq $\backslash R B 3 L Y P \backslash 6-31 G(d) \backslash C 5 H 10 S 2 \backslash D J H 501 \backslash 17-D e C-2003 \backslash 0 \backslash \backslash \#$ RB3 LYP /6-31G* SCF=TIGHT FREQ MAXDISK=52428800 \CH3SCH ( CH=CH2)SCH3C B3LYP $/ 6-31 \mathrm{G}(\mathrm{d})$ freq $\backslash \backslash 0,1 \backslash \mathrm{H},-0.2846308472,-0.7339900972,-1.5261271806 \backslash \mathrm{C}, 0.0$ $541321528,-0.5003610972,-0.5083101806 \backslash \mathrm{C}, 1.0798421528,-1.5312030972,-0$. $0909801806 \backslash \mathrm{C}, 2.4014551528,-1.3686140972,-0.0399311806 \backslash \mathrm{S},-1.4283898472$, $-0.7027240972,0.5884958194 \backslash \mathrm{S}, 0.6630781528,1.2327879028,-0.6732491806 \backslash \mathrm{C}$ $,-2.6768518472,0.2121919028,-0.3792561806 \backslash \mathrm{C}, 1.0441671528,1.6669889028$, $1.0606428194 \backslash \mathrm{H}, 2.8780421528,-0.4255630972,-0.2910601806 \backslash \mathrm{H}, 3.0499541528$ $,-2.1939810972,0.2399298194 \backslash \mathrm{H}, 0.6517721528,-2.5029000972,0.1552808194 \backslash$ $\mathrm{H},-2.3563068472,1.2440369028,-0.5435911806 \backslash \mathrm{H},-2.8694938472,-0.27519709$ $72,-1.3400891806 \backslash \mathrm{H},-3.5967248472,0.2087259028,0.2113778194 \backslash \mathrm{H}, 0.1674821$ $528,1.5029899028,1.6920618194 \backslash \mathrm{H}, 1.8891571528,1.0909079028,1.4441928194$ $\backslash \mathrm{H}, 1.2992671528,2.7299349028,1.0610878194 \backslash \backslash$ Version=DEC-AXP-OSF $/ 1-\mathrm{G} 03 \mathrm{Re}$ vB. 02 \State $=1-A \backslash H F=-992.9007334 \backslash R M S D=8.539 e-09 \backslash R M S F=1.900 e-06 \backslash D i p o l e=-$ $0.0563171,0.03049,-0.0049702 \backslash \mathrm{PG}=\mathrm{C} 01[\mathrm{X}(\mathrm{C} 5 \mathrm{H} 10 \mathrm{~S} 2)] \backslash \backslash @$

\section{$\mathrm{CH}_{3} \mathrm{SCH}\left(\mathrm{CH}=\mathrm{CH}_{2}\right) \mathrm{SCH}_{3}$}

$1 \backslash 1 \backslash G I N C-S C 5 \backslash F O p t \backslash U B 3 L Y P \backslash 6-31 G(d) \backslash C 5 H 9 S 2(2) \backslash D J H 501 \backslash 11-0 c t-2003 \backslash 0 \backslash \backslash \# N \quad U$ B3LYP/6-31G(D) SCF=TIGHT OPT=(CALCFC, TIGHT $)$ MAXDISK=52428800 \CH $3 \mathrm{SC}(\mathrm{CH}$ $=\mathrm{CH} 2) \mathrm{SCH} 3 \mathrm{a}$ radical UB3LYP / 6-31G(d) geom opt $\backslash \backslash 0,2 \backslash \mathrm{C}, 0.4833687989,-0.1$ $825535953,-0.1641271533 \backslash \mathrm{C}, 1.8290604616,-0.4095214907,-0.5293539845 \backslash \mathrm{C}, 2$ $.8479813977,0.5109972587,-0.563831548 \backslash \mathrm{S},-0.5662707171,-1.5888680987,0$. $0769883221 \backslash \mathrm{S},-0.1279381093,1.4668964129,0.052399569 \backslash \mathrm{C},-2.0598342732,-1$ $.1830267966,-0.9103964795 \backslash \mathrm{C},-0.9458441458,1.3949717187,1.6937011188 \backslash \mathrm{H}$, $2.6996667323,1.5445607473,-0.2705072955 \backslash \mathrm{H}, 3.8406904894,0.2173958524,-0$ $.8884508603 \backslash \mathrm{H}, 2.0616553195,-1.4345579941,-0.8160024942 \backslash \mathrm{H},-2.5107370239$ $,-0.2448455172,-0.5810883737 \backslash \mathrm{H},-1.8140913127,-1.121094494,-1.972999183$ $4 \backslash \mathrm{H},-2.7636483689,-2.0031588709,-0.7445903199 \backslash \mathrm{H},-1.7259614747,0.631497$ $5101,1.7159712025 \backslash \mathrm{H},-0.2139778348,1.1971434949,2.4800263894 \backslash \mathrm{H},-1.39464$ $87377,2.3794036749,1.8514829556 \backslash \backslash$ Version=DEC-AXP-OSF / 1-G03RevB.02\Stat 
$\mathrm{e}=2-\mathrm{A} \backslash \mathrm{HF}=-992.2714039 \backslash \mathrm{S} 2=0.775712 \backslash \mathrm{S} 2-1=0 . \backslash \mathrm{S} 2 \mathrm{~A}=0.750186 \backslash \mathrm{RMSD}=7.972 \mathrm{e}-09 \backslash$ $\mathrm{RMSF}=2.998 \mathrm{e}-07 \backslash \mathrm{Dipole}=-0.4434942,0.0804011,0.1340519 \backslash \mathrm{PG}=\mathrm{C} 01 \quad[\mathrm{X}(\mathrm{C} 5 \mathrm{H} 9 \mathrm{~S} 2)$ ]$\backslash \backslash Q$

\section{$\mathrm{CH}_{3} \mathrm{~S}\left(\mathrm{CH}=\mathrm{CH}_{2}\right) \mathrm{C}=\mathrm{S}$}

$1 \backslash 1 \backslash G I N C-S C 5 \backslash F r e q \backslash R B 3 L Y P \backslash 6-31 G(d) \backslash C 4 H 6 S 2 \backslash D J H 501 \backslash 09-M a y-2004 \backslash 1 \backslash \backslash \#$ B3LYP /6-31G* SCF=TIGHT FREQ MAXDISK=39321600 \CH3SC $(\mathrm{CH}=\mathrm{CH} 2)=\mathrm{S}$ B3LYP / 6-31G (d) freq $\backslash \backslash 0,1 \backslash \mathrm{C} \backslash \mathrm{C}, 1, \mathrm{~B} 1 \backslash \mathrm{C}, 2$, B2 , $1, \mathrm{~A} 1 \backslash \mathrm{S}, 1, \mathrm{~B} 3,2, \mathrm{~A} 2,3,180$. , $0 \backslash \mathrm{S}, 1, \mathrm{~B} 4,2, \mathrm{~A} 3,4$, $180 ., 0 \backslash \mathrm{C}, 4, \mathrm{~B} 5,1, \mathrm{~A} 4,2,180.0 \backslash \mathrm{H}, 3, \mathrm{~B} 6,2, \mathrm{~A} 5,1,0 ., 0 \backslash \mathrm{H}, 3, \mathrm{~B} 7,2, \mathrm{~A} 6,7,180 ., 0 \backslash \mathrm{H}$, $2, \mathrm{~B} 8,1, \mathrm{~A} 7,3,180 ., 0 \backslash \mathrm{H}, 6, \mathrm{~B} 9,4, \mathrm{~A} 8,1,180.0 \backslash \mathrm{H}, 6, \mathrm{~B} 10,4, \mathrm{~A} 9,10, \mathrm{D} 1,0 \backslash \mathrm{H}, 6, \mathrm{~B} 10,4$ $, A 9,10,-D 1,0 \backslash \backslash B 1=1.47527344 \backslash B 2=1.33956228 \backslash B 3=1.76659617 \backslash B 4=1.65292417 \backslash$ $\mathrm{B} 5=1.81624076 \backslash \mathrm{B} 6=1.08573393 \backslash \mathrm{B} 7=1.08577945 \backslash \mathrm{B} 8=1.08959291 \backslash \mathrm{B} 9=1.09308945 \backslash$ $\mathrm{B} 10=1.09199509 \backslash \mathrm{A} 1=124.53065567 \backslash \mathrm{A} 2=109.7377846 \backslash \mathrm{A} 3=125.52225877 \backslash \mathrm{A} 4=103.6$ $803153 \backslash A 5=121.23889942 \backslash A 6=120.83458319 \backslash A 7=116.16606423 \backslash A 8=106.01045799$ $\backslash A 9=110.39664309 \backslash D 1=119.52265108 \backslash \backslash$ Version=DEC-AXP-OSF / 1-G03RevB . 05 \Sta $\mathrm{te}=1-\mathrm{A}^{\prime} \backslash \mathrm{HF}=-952.3882006 \backslash \mathrm{RMSD}=9.499 \mathrm{e}-09 \backslash \mathrm{RMSF}=6.996 \mathrm{e}-06 \backslash \mathrm{Dipole}=-0.628360$ $8,0 ., 0.2422241 \backslash \mathrm{PG}=\mathrm{CS}[\mathrm{SG}(\mathrm{C} 4 \mathrm{H} 4 \mathrm{~S} 2), \mathrm{X}(\mathrm{H} 2)] \backslash \backslash @$

\section{$\mathrm{CH}_{3} \mathrm{SC} \cdot(\mathrm{CN}) \mathrm{SCH}_{3}(5)$}

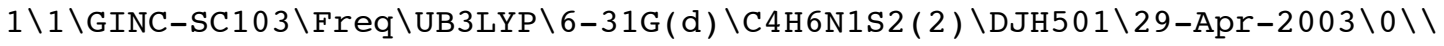
\#N UB3LYP/6-31G(D) SCF=TIGHT FREQ MAXDISK=39321600\\CH3SC(CN)SCH3d rad ical UB3LYP / 6-31G(d) freq $\backslash \backslash 0,2 \backslash \mathrm{C}, 0 ., 0 ., 1.3909831014 \backslash \mathrm{C}, 0 ., 0 .,-0.00177$ $88986 \backslash \mathrm{S}, 0 ., 1.48737,-0.9364158986 \backslash \mathrm{C},-0.95775,2.619045,0.1386721014 \backslash \mathrm{S}, 0$. $,-1.48737,-0.9364158986 \backslash \mathrm{C}, 0.95775,-2.619045,0.1386721014 \backslash \mathrm{H},-1.992213,2$ $.280518,0.2285301014 \backslash \mathrm{H},-0.499302,2.697444,1.1267991014 \backslash \mathrm{H},-0.93062,3.59$ $448,-0.3533868986 \backslash \mathrm{H}, 1.992213,-2.280518,0.2285301014 \backslash \mathrm{H}, 0.499302,-2.6974$ $44,1.1267991014 \backslash \mathrm{H}, 0.93062,-3.59448,-0.3533868986 \backslash \mathrm{N}, 0 ., 0 ., 2.5660191014 \backslash$ $\backslash$ Version=DEC-AXP-OSF $/ 1-G 98 R e v A .11 .3 \backslash$ State $=2-B \backslash H F=-1007.1120674 \backslash \mathrm{S} 2=0.75$ $9884 \backslash \mathrm{S} 2-1=0 . \backslash \mathrm{S} 2 \mathrm{~A}=0.750062 \backslash \mathrm{RMSD}=6.107 \mathrm{e}-09 \backslash \mathrm{RMSF}=4.470 \mathrm{e}-07 \backslash \mathrm{Dipole}=0 ., 0 .,-$ $0.6530448 \backslash \mathrm{PG}=\mathrm{C} 02 \quad[\mathrm{C} 2(\mathrm{C} 1 \mathrm{C} 1 \mathrm{~N} 1), \mathrm{X}(\mathrm{C} 2 \mathrm{H} 6 \mathrm{~S} 2)] \backslash \backslash @$

\section{$\mathrm{CH}_{3} \mathrm{SCH}(\mathrm{CN}) \mathrm{SCH}_{3}$}

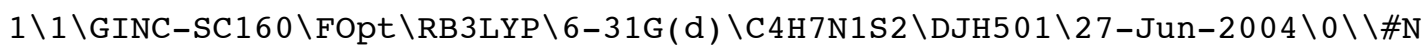
B3LYP/6-31G* SCF=TIGHT OPT MAXDISK=39321600 \CH3SCH(CN)SCH3d B3LYP / 6 $-31 \mathrm{G}(\mathrm{d}) \backslash \backslash 0,1 \backslash \mathrm{C}, 0.2156111309,-0.3806481937,0.5726710689 \backslash \mathrm{C}, 1.6749493252$, $-0.3723893247,0.5237508527 \backslash \mathrm{S},-0.3514477731,1.3678645895,0.8129334278 \backslash \mathrm{S}$ $,-0.4944245279,-1.2902365825,-0.8732613703 \backslash \mathrm{C},-2.1059866343,1.047783498$ $2,1.201115454 \backslash \mathrm{C}, 0.2317869576,-0.3442074892,-2.2564143791 \backslash \mathrm{H},-0.10447469$ $19,-0.9684150507,1.4395252678 \backslash \mathrm{N}, 2.8333574203,-0.3557759993,0.439583949$ $6 \backslash \mathrm{H},-2.5826133741,0.4858173859,0.3940373775 \backslash \mathrm{H},-2.2131266262,0.51349706$ $21,2.1498771422 \backslash \mathrm{H},-2.5834253016,2.0271112795,1.2890856843 \backslash \mathrm{H}, 1.32029221$ $64,-0.4413767331,-2.2657773305 \backslash \mathrm{H},-0.0558635201,0.7082115292,-2.1943696$ $316 \backslash \mathrm{H},-0.1784985043,-0.7796925329,-3.1709570557 \backslash \backslash V e r s i o n=D E C-A X P-O S F / 1$ -G03RevB.05 $\backslash$ State $=1-A \backslash H F=-1007.7426907 \backslash R M S D=3.851 e-09 \backslash R M S F=2.199 e-05 \backslash D$ ipole $=-1.5272915,0.0786747,-0.12118 \backslash \mathrm{PG}=\mathrm{C} 01 \quad[\mathrm{X}(\mathrm{C} 4 \mathrm{H} 7 \mathrm{~N} 1 \mathrm{~S} 2)] \backslash \backslash @$

\section{$\mathrm{CH}_{3} \mathrm{~S}(\mathrm{CN}) \mathrm{C}=\mathrm{S}$}

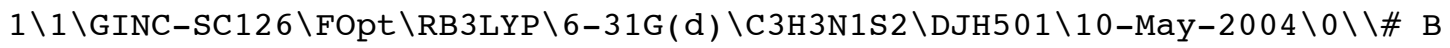
3LYP/6-31G* SCF=TIGHT OPT=TIGHT MAXDISK=39321600 IOP $(1 / 8=10) \backslash \backslash \mathrm{CH} 3 \mathrm{SC}(\mathrm{CN}$ ) Sb B3LYP / 6-31G(d) geom opt \\0,1\C,1.7143130431,-0.4903533238,-0.000 $0026937 \backslash \mathrm{C}, 0.405329302,0.1041091147,-0.0000016782 \backslash \mathrm{S},-0.8432374325,-1.12$ $90645757,0.0000193385 \backslash \mathrm{C},-2.3647846875,-0.1341256382,0.0000040713 \backslash \mathrm{S}, 0.2$ $21723625,1.7406005545,-0.0000181688 \backslash \mathrm{H},-3.1883355575,-0.8523386291,-0.0$ 
$000190697 \backslash \mathrm{H},-2.4091215943,0.4936780571,0.8925537675 \backslash \mathrm{H},-2.4090883069,0$. $4936981649,-0.8925330863 \backslash N, 2.7745172047,-0.9710563101,-0.0000026461 \backslash \backslash V$ ersion=DEC-AXP-OSF /1-G03RevB.05 \State $=1-A \backslash H F=-967.2190127 \backslash \mathrm{RMSD}=7.862 \mathrm{e}-$ $09 \backslash \mathrm{RMSF}=2.473 e-06 \backslash \mathrm{Dipole}=-1.8143545,-0.0479982,0.0000052 \backslash \mathrm{PG}=\mathrm{C} 01] \mathrm{X}(\mathrm{C} 3 \mathrm{H}$ $3 N 1 S 2$ ) $] \backslash \backslash[$

\section{$\mathrm{CH}_{3} \mathrm{SC} \cdot\left(\mathrm{CF}_{3}\right) \mathrm{SCH}_{3}(6)$}

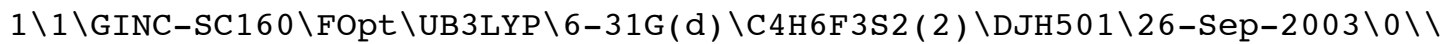
\#N UB3LYP/6-31G(D) SCF=TIGHT OPT=CALCFC MAXDISK=39321600\\CH3SC(CF3)SC H3 radical UB3LYP / 6-31G(d) geom opt \\0,2\F,-1.0471802293,1.525585842 $6,-1.015434 \backslash \mathrm{C},-0.2091711696,1.1281099686,-0.017138 \backslash \mathrm{C}, 0.10325005,-0.332$ $4039845,-0.076694 \backslash \mathrm{S}, 1.6484101431,-0.9523747522,-0.608042 \backslash \mathrm{C}, 2.835255051$ $4,-0.3419965739,0.660132 \backslash \mathrm{S},-1.1071517692,-1.5357321664,0.274576 \backslash \mathrm{C},-2.7$ $2002889,-0.7319804088,-0.070076 \backslash \mathrm{H},-2.7932929324,-0.4497534198,-1.12146$ $6 \backslash \mathrm{H},-2.8675600212,0.140908569,0.566798 \backslash \mathrm{F}, 0.8965477154,1.8936931348,-0$. $119665 \backslash \mathrm{F},-0.8305872208,1.4690158752,1.142202 \backslash \mathrm{H}, 2.5690871098,-0.7306406$ $139,1.64556 \backslash \mathrm{H}, 2.8669508877,0.7474174309,0.673303 \backslash \mathrm{H}, 3.8124831104,-0.734$ $612427,0.365461 \backslash \mathrm{H},-3.4726507763,-1.4886305219,0.164543 \backslash \backslash$ Version=DEC-AX $\mathrm{P}-\mathrm{OSF} / 1-\mathrm{G} 03 \mathrm{RevB} .02 \backslash \mathrm{State}=2-\mathrm{A} \backslash \mathrm{HF}=-1251.8950979 \backslash \mathrm{S} 2=0.754673 \backslash \mathrm{S} 2-1=0 . \backslash \mathrm{S} 2 \mathrm{~A}=$ $0.750016 \backslash \mathrm{RMSD}=7.279 \mathrm{e}-09 \backslash \mathrm{RMSF}=3.241 \mathrm{e}-06 \backslash \mathrm{Dipole}=-0.1469445,-0.1666866,0$. $313772 \backslash$ Polar $=117.9609358,5.9838447,69.8165001,-1.3695049,-0.1431546,57$ $.7037004 \backslash \mathrm{PG}=\mathrm{C} 01 \quad[\mathrm{X}(\mathrm{C} 4 \mathrm{H} 6 \mathrm{~F} 3 \mathrm{~S} 2)] \backslash \backslash \mathrm{a}$

\section{$\mathrm{CH}_{3} \mathrm{SCH}\left(\mathrm{CF}_{3}\right) \mathrm{SCH}_{3}$}

$1 \backslash 1 \backslash G I N C-S C 160 \backslash$ Freq $\backslash R B 3 L Y P \backslash 6-31 G(d) \backslash C 4 H 7 F 3 S 2 \backslash D J H 501 \backslash 05-O c t-2003 \backslash 0 \backslash \backslash \# N$ B3LYP $/ 6-31 \mathrm{G}(\mathrm{D}) \mathrm{SCF}=\mathrm{TIGHT} \mathrm{OPT}=(\mathrm{CALCALL}, \mathrm{TIGHT}) \quad$ IOP $(1 / 8=10)$ MAXDISK=52428 $800 \backslash \backslash \mathrm{CH} 3 \mathrm{SCH}(\mathrm{CF} 3) \mathrm{SCH} 3 \mathrm{~B} 3 \mathrm{LYP} / 6-31 \mathrm{G}(\mathrm{d})$ geom opt $\backslash \backslash 0,1 \backslash \mathrm{C}, 0.0802516344,1.2$ $657139969,0.0560706089 \backslash \mathrm{C}, 0.0481511844,-0.1434017835,-0.5473652907 \backslash \mathrm{S},-1$ $.6560288589,-0.7520203104,-0.8385127466 \backslash \mathrm{C},-2.2432287033,-1.1660246643$, $0.8453145567 \backslash \mathrm{S}, 1.0905948667,-1.3368049268,0.3943014998 \backslash \mathrm{C}, 2.736684887,-$ $1.0181886506,-0.3402373061 \backslash \mathrm{F},-0.437757138,1.311340682,1.2981799913 \backslash \mathrm{F},-$ $0.6046087612,2.1371354189,-0.7079367641 \backslash \mathrm{F}, 1.3521863973,1.7264403751,0$. $1327241271 \backslash \mathrm{H}, 3.0854227897,-0.0087604087,-0.1184977301 \backslash \mathrm{H}, 2.723497889,-1$ $.1878948932,-1.4210952192 \backslash \mathrm{H}, 0.4636798452,-0.0529797823,-1.5559878687 \backslash \mathrm{H}$ $,-2.3030420305,-0.2813666413,1.4798759284 \backslash \mathrm{H},-1.6110690258,-1.926907876$ $9,1.3096960121 \backslash \mathrm{H},-3.2450852846,-1.5804834837,0.7048471129 \backslash \mathrm{H}, 3.41400119$ $55,-1.7432447929,0.1191401126 \backslash \backslash$ Version=DEC-AXP-OSF / 1-G03RevB.02 $\backslash$ State= $1-\mathrm{A} \backslash \mathrm{HF}=-1252.5406768 \backslash \mathrm{RMSD}=3.127 \mathrm{e}-09 \backslash \mathrm{RMSF}=5.646 \mathrm{e}-06 \backslash \mathrm{Dipole}=0.4164394,-0$ $.3688252,-0.0859871 \backslash \mathrm{PG}=\mathrm{C} 01[\mathrm{X}(\mathrm{C} 4 \mathrm{H} 7 \mathrm{~F} 3 \mathrm{~S} 2)] \backslash \backslash @$

\section{$\mathrm{CH}_{3} \mathrm{~S}\left(\mathrm{CF}_{3}\right) \mathrm{C}=\mathrm{S}$}

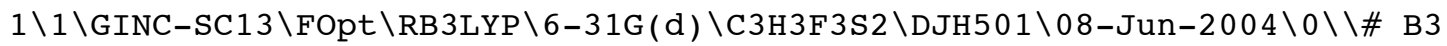
LYP/6-31G* SCF=TIGHT OPT=TIGHT MAXDISK=65536000\\CH3SC(CF3)Se B3LYP / 6-31G(d) geom opt $\backslash \backslash 0,1 \backslash S, 0.1600668171,0,,-1.8994631176 \backslash \mathrm{C},-0.0590038526$ $, 0 .,-0.2784483338 \backslash \mathrm{C}, 1.1623100886,0 ., 0.6593512747 \backslash \mathrm{S},-1.5641330296,0 ., 0$. $606078134 \backslash \mathrm{C},-2.786206208,0 .,-0.7384060645 \backslash \mathrm{H},-3.7647348693,0 .,-0.252024$ $442 \backslash \mathrm{H},-2.6700872521,0.8913530623,-1.358775166 \backslash \mathrm{H},-2.6700872521,-0.89135$ $30623,-1.358775166 \backslash \mathrm{F}, 0.7928951805,0.1 .9602329358 \backslash \mathrm{F}, 1.9184062212,-1.08$ $75114514,0.4537031573 \backslash \mathrm{F}, 1.9184062212,1.0875114514,0.4537031573 \backslash \backslash$ Versio $\mathrm{n}=\mathrm{DEC}-\mathrm{AXP}-\mathrm{OSF} / 1-\mathrm{G} 03 \mathrm{RevB} .05 \backslash \mathrm{State}=1-\mathrm{A}^{\prime} \backslash \mathrm{HF}=-1212.0139559 \backslash \mathrm{RMSD}=5.407 \mathrm{e}-09 \backslash$ $\mathrm{RMSF}=7.326 \mathrm{e}-06 \backslash \mathrm{Dipole}=-1.238597,0 ., 0.0073576 \backslash \mathrm{PG}=\mathrm{CS} \quad[\mathrm{SG}(\mathrm{C} 3 \mathrm{H} 1 \mathrm{~F} 1 \mathrm{~S} 2), \mathrm{X}(\mathrm{H} 2 \mathrm{~F}$ 2) $] \backslash \backslash @$ 


\section{$\mathrm{CH}_{3} \mathrm{SC} \cdot\left(\mathrm{NH}_{2}\right) \mathrm{SCH}_{3}(7)$}

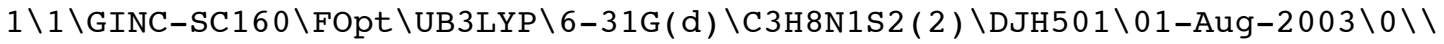
\#N UB3LYP/6-31G(D) SCF=TIGHT OPT=TIGHT MAXDISK=39321600 \CH3SC (NH2) SCH 3 a radical UB3LYP / 6-31G(d) geom opt $\backslash \backslash 0,2 \backslash N,-2.0317724087,0.023730957$ $8,0.1181493798 \backslash \mathrm{C},-0.6727873925,-0.0252932807,-0.2151600701 \backslash \mathrm{S}, 0.2106355$ $736,1.4958584317,-0.2872708422 \backslash \mathrm{C}, 0.6455286398,1.8452679886,1.478640630$ $7 \backslash \mathrm{S}, 0.1066825821,-1.5733978448,0.1803475471 \backslash \mathrm{C}, 1.2683893093,-1.73281006$ $66,-1.2260941018 \backslash \mathrm{H}, 1.8951315145,-0.8403767838,-1.2950878875 \backslash \mathrm{H}, 0.729506$ $5072,-1.8840205046,-2.1643916307 \backslash \mathrm{H}, 1.2107573531,1.0074665523,1.8937668$ $431 \backslash \mathrm{H},-0.2536127284,2.0235064462,2.0725576755 \backslash \mathrm{H}, 1.2698295127,2.7434614$ $588,1.4769111911 \backslash \mathrm{H},-2.565608069,-0.7914144382,-0.1709295489 \backslash \mathrm{H},-2.48407$ $31237,0.8960625765,-0.1352193787 \backslash \mathrm{H}, 1.8966020637,-2.6031592507,-1.01819$ $89547 \backslash \backslash$ Version $=$ DEC-AXP-OSF $/ 1-G 03 R$ RevB . 02 \State $=2-A \backslash H F=-970.2140204 \backslash S 2=0$ $.754391 \backslash \mathrm{S} 2-1=0 . \backslash \mathrm{S} 2 \mathrm{~A}=0.750014 \backslash \mathrm{RMSD}=5.886 \mathrm{e}-09 \backslash \mathrm{RMSF}=6.142 \mathrm{e}-06 \backslash \mathrm{Dipole}=-0.3$ $154557,0.0328513,-0.1508421 \backslash \mathrm{PG}=\mathrm{C} 01[\mathrm{X}(\mathrm{C} 3 \mathrm{H} 8 \mathrm{~N} 1 \mathrm{~S} 2)] \backslash \backslash @$

\section{$\mathrm{CH}_{3} \mathrm{SCH}\left(\mathrm{NH}_{2}\right) \mathrm{SCH}_{3}$}

$1 \backslash 1 \backslash G I N C-S C 3 \backslash F O p t \backslash R B 3 L Y P \backslash 6-31 G(d) \backslash C 3 H 9 N 1 S 2 \backslash D J H 501 \backslash 17-D e c-2003 \backslash 0 \backslash \backslash \# N$ B3 $\mathrm{LYP} / 6-31 \mathrm{G}(\mathrm{D}) \mathrm{SCF}=\mathrm{TIGHT} \mathrm{OPT}=(\mathrm{CALCFC}, \mathrm{TIGHT}) \quad \mathrm{MAXDISK}=52428800 \mathrm{IOP}(1 / 8=10)$ $\backslash \backslash \mathrm{CH} 3 \mathrm{SCH}(\mathrm{NH} 2) \mathrm{SCH} 3 \mathrm{~b}$ B3LYP / 6-31G(d) geom opt $\backslash \backslash 0,1 \backslash \mathrm{N}, 0.6607453834,1.940$ $133127,-0.1998799073 \backslash \mathrm{C}, 0.0416817984,0.6876949576,-0.5641805356 \backslash \mathrm{S},-1.43$ $29213371,0.3499511602,0.5416553793 \backslash \mathrm{C},-2.1797681096,-1.1205297643,-0.24$ $46331986 \backslash \mathrm{S}, 1.2533739746,-0.6921488868,-0.6622087474 \backslash \mathrm{C}, 1.895318992,-0.7$ $278001035,1.0472248695 \backslash \mathrm{H},-0.3301799087,0.7679383259,-1.5897206285 \backslash \mathrm{H}, 1$. $0708439652,-0.7609248001,1.7653864321 \backslash \mathrm{H}, 2.546743217,0.1269603507,1.251$ $0819682 \backslash \mathrm{H}, 0.9797581794,1.9184835544,0.7667164496 \backslash \mathrm{H},-0.0187791126,2.696$ $5818441,-0.2721888541 \backslash \mathrm{H},-1.4587409531,-1.9406394978,-0.2855872819 \backslash \mathrm{H},-2$ $.539391831,-0.8923290264,-1.2527578418 \backslash \mathrm{H},-3.030750379,-1.4144077187,0$. $3757290232 \backslash \mathrm{H}, 2.4846408531,-1.6436218327,1.1388871613 \backslash \backslash$ Version=DEC-AXP$\mathrm{OSF} / 1-\mathrm{G} 03 \mathrm{RevB} .02 \backslash \mathrm{State}=1-\mathrm{A} \backslash \mathrm{HF}=-970.8545483 \backslash \mathrm{RMSD}=4.712 \mathrm{e}-09 \backslash \mathrm{RMSF}=1.433 \mathrm{e}-$ $06 \backslash \mathrm{Dipole}=-0.2454906,-0.0187076,0.3452151 \backslash \mathrm{PG}=\mathrm{C} 01 \quad[\mathrm{X}(\mathrm{C} 3 \mathrm{H} 9 \mathrm{~N} 1 \mathrm{~S} 2)] \backslash \backslash @$

\section{$\mathrm{CH}_{3} \mathrm{~S}\left(\mathrm{NH}_{2}\right) \mathbf{C}=\mathbf{S}$}

$1 \backslash 1 \backslash G I N C-S C 119 \backslash F r e q \backslash R B 3 L Y P \backslash 6-31 G(d) \backslash C 2 H 5 N 1 S 2 \backslash D J H 501 \backslash 16-M a y-2004 \backslash 1 \backslash \backslash \# B$ 3LYP/6-31G* SCF=TIGHT FREQ MAXDISK=52428800\\CH3SC(NH2)S B3LYP / 6-31G (d) freq $\backslash \backslash 0,1 \backslash N \backslash C, 1, B 1 \backslash S, 2, B 2,1, A 1 \backslash S, 2, B 3,1, A 2,3,180$. , 0\C , 3, B4, $2, A 3,1$, $180 ., 0 \backslash \mathrm{H}, 5, \mathrm{~B} 5,3, \mathrm{~A} 4,2,180.0 \backslash \mathrm{H}, 5, \mathrm{~B} 6,3, \mathrm{~A} 5,6, \mathrm{D} 1,0 \backslash \mathrm{H}, 5, \mathrm{~B} 6,3, \mathrm{~A} 5,6,-\mathrm{D} 1,0 \backslash \mathrm{H}, 1$ $, \mathrm{B} 7,2, \mathrm{~A} 6,3,0 ., 0 \backslash \mathrm{H}, 1, \mathrm{~B} 8,2, \mathrm{~A} 7,9,180 ., 0 \backslash \backslash \mathrm{B} 1=1.35086232 \backslash \mathrm{B} 2=1.78936764 \backslash \mathrm{B} 3=1$ $.66174545 \backslash B 4=1.81922951 \backslash B 5=1.09354712 \backslash B 6=1.09091636 \backslash B 7=1.011685 \backslash B 8=1.0$ $1047704 \backslash \mathrm{A} 1=111.06777739 \backslash \mathrm{A} 2=123.07335073 \backslash \mathrm{A} 3=102.40560811 \backslash \mathrm{A} 4=105.4566422$ $7 \backslash A 5=110.47552997 \backslash A 6=122.66517381 \backslash A 7=118.51199776 \backslash D 1=119.17507094 \backslash \backslash$ Ver sion=DEC-AXP-OSF $/ 1-G 03 R e v B .05 \backslash$ State $=1-A^{\prime} \backslash \mathrm{HF}=-930.3575603 \backslash \mathrm{RMSD}=1.856 \mathrm{e}-0$ $9 \backslash \mathrm{RMSF}=3.231 \mathrm{e}-06 \backslash \mathrm{Dipole}=0.8655404,0,,-0.7220802 \backslash \mathrm{PG}=\mathrm{CS}[\mathrm{SG}(\mathrm{C} 2 \mathrm{H} 3 \mathrm{~N} 1 \mathrm{~S} 2), \mathrm{X}($ H2 ) ] \@

\section{$\mathrm{CH}_{3} \mathrm{SC} \cdot\left(\mathrm{CH}_{3}\right) \mathrm{S}-\mathrm{CH}_{3}(8)$}

$1 \backslash 1 \backslash$ GINC-SC65 $\backslash$ FOpt \UB3LYP \6-31G(d) \C4H9S2 ( 2$) \backslash M L C 501 \backslash 16-J u 1-2002 \backslash 1 \backslash \backslash \# N$ UB3LYP/6-31G(D) FOPT=(Z-MATRIX, TIGHT, MAXCYC=100) FREQ MAXDISK=13107200 $0 \mathrm{SCF}=(\mathrm{TIGHT}, \mathrm{MAXCYC}=100) \mathrm{GEOM}=\mathrm{CHECK}$ GUESS $=$ READ $\backslash \backslash$ mescmesme $-\mathrm{c} 1 \mathrm{~b}-6 \mathrm{dub} 3 \backslash \backslash 0$ , $2 \backslash \mathrm{H} \backslash \mathrm{C}, 1, \mathrm{~B} 1 \backslash \mathrm{H}, 2, \mathrm{~B} 2,1, \mathrm{~A} 1 \backslash \mathrm{S}, 2, \mathrm{~B} 3,1, \mathrm{~A} 2,3, \mathrm{D} 1,0 \backslash \mathrm{C}, 4, \mathrm{~B} 4,2, \mathrm{~A} 3,1, \mathrm{D} 2,0 \backslash \mathrm{S}, 5, \mathrm{~B} 5,4$ , $\mathrm{A} 4,2, \mathrm{D} 3,0 \backslash \mathrm{C}, 6, \mathrm{~B} 6,5, \mathrm{~A} 5,4,-\mathrm{D} 4,0 \backslash \mathrm{H}, 7, \mathrm{~B} 7,6, \mathrm{~A} 6,5, \mathrm{D} 5,0 \backslash \mathrm{H}, 7, \mathrm{~B} 8,6, \mathrm{~A} 7,8, \mathrm{D} 6,0 \backslash \mathrm{H}$ $, 7, \mathrm{~B} 9,6, \mathrm{~A} 8,8, \mathrm{D} 7,0 \backslash \mathrm{C}, 5, \mathrm{~B} 10,4, \mathrm{~A} 9,2, \mathrm{D} 8,0 \backslash \mathrm{H}, 2, \mathrm{~B} 11,1, \mathrm{~A} 10,3, \mathrm{D} 9,0 \backslash \mathrm{H}, 11, \mathrm{~B} 12,5$, $\mathrm{A} 11,4, \mathrm{D} 10,0 \backslash \mathrm{H}, 11, \mathrm{~B} 13,5, \mathrm{~A} 12,4, \mathrm{D} 11,0 \backslash \mathrm{H}, 11, \mathrm{~B} 14,5, \mathrm{~A} 13,4, \mathrm{D} 12,0 \backslash \backslash \mathrm{B} 1=1.092657$ $93 \backslash \mathrm{B} 2=1.09285939 \backslash \mathrm{B} 3=1.83510395 \backslash \mathrm{B} 4=1.77013049 \backslash \mathrm{B} 5=1.75804982 \backslash \mathrm{B} 6=1.832220$ 
$08 \backslash \mathrm{B} 7=1.09257315 \backslash \mathrm{B} 8=1.09085787 \backslash \mathrm{B} 9=1.09328827 \backslash \mathrm{B} 10=1.50447696 \backslash \mathrm{B} 11=1.0934$ $6331 \backslash \mathrm{B} 12=1.09613505 \backslash \mathrm{B} 13=1.09553397 \backslash \mathrm{B} 14=1.10178689 \backslash \mathrm{A} 1=110.0297286 \backslash \mathrm{A} 2=11$ $0.82443615 \backslash A 3=102.23773298 \backslash A 4=118.51568336 \backslash A 5=103.96848806 \backslash A 6=110.4950$ $1693 \backslash A 7=111.21266523 \backslash A 8=105.91951214 \backslash A 9=120.43443778 \backslash A 10=109.33705389 \backslash$ $\mathrm{A} 11=111.15729093 \backslash \mathrm{A} 12=110.74166016 \backslash \mathrm{A} 13=112.20742397 \backslash \mathrm{D} 1=-124.06148497 \backslash \mathrm{D} 2$ $=60.56470818 \backslash \mathrm{D} 3=-144.49451711 \backslash \mathrm{D} 4=-48.977946 \backslash \mathrm{D} 5=60.52936234 \backslash \mathrm{D} 6=-122.478$ $49479 \backslash D 7=118.55770463 \backslash D 8=62.40591497 \backslash D 9=119.28641302 \backslash D 10=-44.11186517 \backslash$ $\mathrm{D} 11=-164.00422423 \backslash \mathrm{D} 12=75.46393503 \backslash \backslash$ Version=DEC-AXP-OSF / 1-G98RevA.11.3\ $\mathrm{HF}=-954.1760596 \backslash \mathrm{S} 2=0.755169 \backslash \mathrm{S} 2-1=0 . \backslash \mathrm{S} 2 \mathrm{~A}=0.75002 \backslash \mathrm{RMSD}=5.132 \mathrm{e}-09 \backslash \mathrm{RMSF}=2$. $815 e-05 \backslash \mathrm{Dipole}=0.2098476,0.5512682,-0.4657697 \backslash \mathrm{PG}=\mathrm{C} 01[\mathrm{X}(\mathrm{C} 4 \mathrm{H} 9 \mathrm{~S} 2)] \backslash \backslash @$

\section{$\mathrm{CH}_{3} \mathrm{SCH}\left(\mathrm{CH}_{3}\right) \mathrm{S}-\mathrm{CH}_{3}$}

$1 \backslash 1 \backslash$ GINC-SC50 $\backslash$ FOpt $\backslash R B 3 L Y P \backslash 6-31 G(d) \backslash C 4 H 10 S 2 \backslash M L C 501 \backslash 16-J a n-2003 \backslash 0 \backslash \backslash \# N$ B3 LYP/6-31G(D) OPT FREQ MAXDISK=39321600\\ralk $z=$ me $r=$ me-c $1 b-6$ dub3 $\backslash \backslash 0,1 \backslash$ $\mathrm{H},-0.8520756361,0.8033167342,2.6879932569 \backslash \mathrm{C}, \overline{0} .2370 \overline{2} 8745,0.7400719558,2$ $.5967512165 \backslash \mathrm{H}, 0.66012251,1.7461819529,2.5241712482 \backslash \mathrm{S}, 0.7265755304,-0.3$ $135602287,1.182376267 \backslash \mathrm{C},-0.0981477987,0.5907986636,-0.2087141703 \backslash \mathrm{S},-0$. $2432081378,-0.5178018206,-1.6643786617 \backslash \mathrm{C},-1.5184006495,-1.6897698027,-$ $1.08384029 \backslash \mathrm{H},-2.4735833353,-1.1832268548,-0.9147693289 \backslash \mathrm{H},-1.1948363437$ $,-2.1931652975,-0.1698746482 \backslash \mathrm{H},-1.6462270616,-2.4302742312,-1.87782798$ $13 \backslash \mathrm{C}, 0.6612927142,1.8492910624,-0.6303946624 \backslash \mathrm{H}, 0.6349852607,0.26071070$ $74,3.4953485823 \backslash \mathrm{H}, 0.7958825981,2.5207757598,0.2239981057 \backslash \mathrm{H}, 0.107849916$ $6,2.3888619535,-1.407314693 \backslash \mathrm{H}, 1.6524532115,1.5937434843,-1.0176689327 \backslash$ $\mathrm{H},-1.1090874676,0.8525173058,0.1251701442 \backslash \backslash$ Version=DEC-AXP-OSF / 1-G98Re $\mathrm{VA} .11 .3 \backslash \mathrm{HF}=-954.8250473 \backslash \mathrm{RMSD}=5.044 \mathrm{e}-09 \backslash \mathrm{RMSF}=1.205 \mathrm{e}-05 \backslash \mathrm{Dipole}=-0.682714$ $7,0.4700445,0.5466841 \backslash \mathrm{PG}=\mathrm{C} 01 \quad[\mathrm{X}(\mathrm{C} 4 \mathrm{H} 10 \mathrm{~S} 2)] \backslash \backslash @$

\section{$\mathrm{S}=\mathrm{C}\left(\mathrm{CH}_{3}\right) \mathrm{SCH}_{3}$}

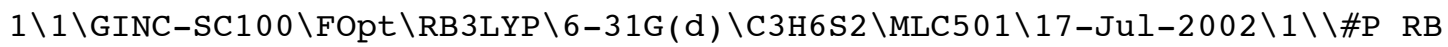
$3 \mathrm{LYP} / 6-31 \mathrm{G} * \mathrm{FOPT}=($ MAXCYC $=150, \mathrm{Z}-\mathrm{MATRIX}) \quad \mathrm{MAXDISK}=131072000 \mathrm{FREQ} \quad \mathrm{SCF}=\mathrm{TIGH}$

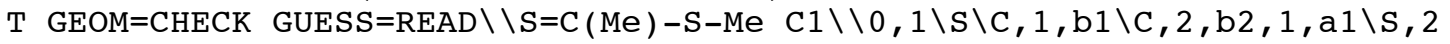
, b3 , $1, \mathrm{a} 2,3, \mathrm{dh} 3,0 \backslash \mathrm{C}, 4, \mathrm{~b} 4,2, \mathrm{a} 3,1, \mathrm{dh} 4,0 \backslash \mathrm{H}, 5, \mathrm{~b} 5,4, \mathrm{a} 4,2, \mathrm{dh} 5,0 \backslash \mathrm{H}, 5, \mathrm{~b} 6,4, \mathrm{a} 5,2$ , dh $1,0 \backslash \mathrm{H}, 5, \mathrm{~b} 6 \mathrm{a}, 4, \mathrm{a} 5 \mathrm{a}, 2,-\mathrm{dh} 1 \mathrm{a}, 0 \backslash \mathrm{H}, 3, \mathrm{~b} 7,2, \mathrm{a} 6,1, \mathrm{dh} 6,0 \backslash \mathrm{H}, 3, \mathrm{~b} 8,2, \mathrm{a} 7,1, \mathrm{dh} 2,0$ $\backslash \mathrm{H}, 3, \mathrm{~b} 8 \mathrm{a}, 2, \mathrm{a} 7 \mathrm{a}, 1,-\mathrm{dh} 2 \mathrm{a}, 0 \backslash \backslash \mathrm{b} 1=1.64599636 \backslash \mathrm{b} 2=1.5148461 \backslash \mathrm{b} 3=1.75511783 \backslash \mathrm{b} 4=$ $1.81794969 \backslash \mathrm{b} 5=1.09307671 \backslash \mathrm{b} 6=1.0919738 \backslash \mathrm{b} 6 \mathrm{a}=1.09197036 \backslash \mathrm{b} 7=1.09324611 \backslash \mathrm{b} 8=$ $1.09537804 \backslash \mathrm{b} 8 \mathrm{a}=1.0962657 \backslash \mathrm{a} 1=122.84648556 \backslash \mathrm{a} 2=125.75342086 \backslash \mathrm{a} 3=103.776055$ $3 \backslash a 4=106.19073532 \backslash a 5=110.33849971 \backslash a 5 a=110.27255399 \backslash a 6=109 \cdot 69980988 \backslash a 7=$ $112.81878244 \backslash a 7 a=109.03573022 \backslash \mathrm{dh} 1=60.70823437 \backslash \mathrm{dh} 1 \mathrm{a}=60.13720525 \backslash \mathrm{dh} 2=167$ $.56947484 \backslash \mathrm{dh} 2 \mathrm{a}=72.03339216 \backslash \mathrm{dh} 3=181.23401229 \backslash \mathrm{dh} 4=0.90237034 \backslash \mathrm{dh} 5=180.305$ $77272 \backslash \mathrm{dh} 6=45.4338183 \backslash \backslash$ Version=DEC-AXP-OSF $/ 1-G 98 \mathrm{RevA} .11 .3 \backslash \mathrm{HF}=-914.30737$ $69 \backslash \mathrm{RMSD}=3.465 \mathrm{e}-10 \backslash \mathrm{RMSF}=1.676 \mathrm{e}-06 \backslash \mathrm{Dipole}=-0.3544958,-0.0417535,0.694415$ $9 \backslash \mathrm{PG}=\mathrm{C} 01 \quad[\mathrm{X}(\mathrm{C} 3 \mathrm{H} 6 \mathrm{~S} 2)] \backslash \backslash @$

\section{$\mathrm{CH}_{3} \mathrm{SC} \cdot(\mathrm{Ph}) \mathrm{S}-\mathrm{CH}_{3}(9)$}

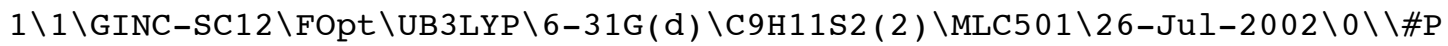
B3LYP $/ 6-31 \mathrm{G} * \mathrm{OPT}=(\mathrm{MAXCYC}=100) \mathrm{SCF}=(\mathrm{MAXCYC}=100) \quad \mathrm{FREQ}$ MAXDISK $=131072000$ $\backslash \backslash \mathrm{rrad} z=\mathrm{ph} r=\mathrm{me}-\mathrm{c} 1 \mathrm{c}-6 \mathrm{duhf}--\mathrm{C} 2 \backslash \backslash 0,2 \backslash \mathrm{C}, 0,0 .,-0.5436243969 \backslash \mathrm{C}, 0 ., 0 ., 0.89$ $993655 \overline{5} 8 \backslash \mathrm{C}, \overline{1} .2045767983,-0.1407684102,1.6447473811 \backslash \mathrm{C},-1.2045767983,0.1$ $407684102,1.6447473811 \backslash \mathrm{C}, 1.1980023223,-0.1372926446,3.0327870849 \backslash \mathrm{C},-1$. $1980023223,0.1372926446,3.0327870849 \backslash \mathrm{H}, 2.1407212827,-0.2659311883,1.11$ $24152303 \backslash \mathrm{H},-2.1407212827,0.2659311883,1.1124152303 \backslash \mathrm{H}, 2.1367874102,-0.2$ $51386417,3.5689285696 \backslash \mathrm{H},-2.1367874102,0.251386417,3.5689285696 \backslash \mathrm{C}, 0 ., 0$.

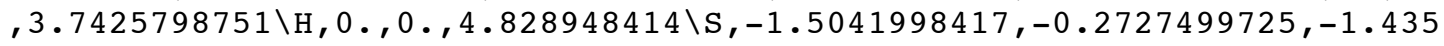
$6947229 \backslash \mathrm{C},-1.5483731257,1.1133863252,-2.639460946 \backslash \mathrm{H},-2.4327150559,0.94$ $51928411,-3.2601525901 \backslash \mathrm{H},-1.6417073267,2.0701943282,-2.120752452 \backslash \mathrm{H},-0$. $656494302,1.1210366726,-3.2689146201 \backslash \mathrm{S}, 1.5041998417,0.2727499725,-1.43$ $56947229 \backslash \mathrm{C}, 1.5483731257,-1.1133863252,-2.639460946 \backslash \mathrm{H}, 2.4327150559,-0.9$ 
$451928411,-3.2601525901 \backslash \mathrm{H}, 1.6417073267,-2.0701943282,-2.120752452 \backslash \mathrm{H}, 0$. $656494302,-1.1210366726,-3.2689146201 \backslash$ VVersion=DEC-AXP-OSF / 1-G98RevA. 1 $1.3 \backslash$ State $=2-B \backslash H F=-1145.9239982 \backslash S 2=0.772251 \backslash S 2-1=0 . \backslash S 2 A=0.750367 \backslash R M S D=6$ $.432 \mathrm{e}-09 \backslash \mathrm{RMSF}=1.039 \mathrm{e}-05 \backslash \mathrm{Dipole}=0,, 0,-0.434059 \backslash \mathrm{PG}=\mathrm{C} 02$ [C2 $(\mathrm{C} 1 \mathrm{C} 1 \mathrm{C} 1 \mathrm{H} 1), \mathrm{X}($ C6H10S2) ] \\@

\section{$\mathrm{CH}_{3} \mathrm{SCH}(\mathrm{Ph}) \mathrm{S}-\mathrm{CH}_{3}$}

$1 \backslash 1 \backslash \mathrm{GINC}-\mathrm{SC} 50 \backslash \mathrm{FOpt} \backslash \mathrm{RB} 3 \mathrm{LYP} \backslash 6-31 \mathrm{G}(\mathrm{d}) \backslash \mathrm{C} 9 \mathrm{H} 12 \mathrm{~S} 2 \backslash \mathrm{MLC} 501 \backslash 16-\mathrm{Jan}-2003 \backslash 0 \backslash \backslash \mathrm{H}$ B3

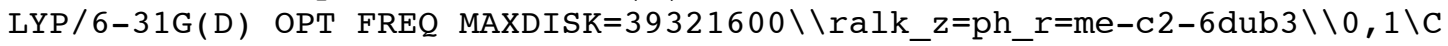
$,-0.58192849,-0.0676167262,0.6559765072 \backslash \mathrm{C}, 0 . \overline{8} 6926 \overline{5} 9739,0.0219992291,0$. $1701840044 \backslash \mathrm{C}, 1.4857483314,1.2596861061,-0.0639221236 \backslash \mathrm{C}, 1.6453478345,-1$ $.1399199407,0.0253093128 \backslash \mathrm{C}, 2.827536998,1.3344024726,-0.4400288414 \backslash \mathrm{C}, 2$. $9867423773,-1.0640643596,-0.350976908 \backslash \mathrm{H}, 0.912625679,2.1721030051,0.063$ $0616357 \backslash \mathrm{H}, 1.20024715,-2.1111659146,0.2126653432 \backslash \mathrm{H}, 3.2788869284,2.30745$ $02028,-0.6159236246 \backslash \mathrm{H}, 3.56335567,-1.9793611551,-0.4563357395 \backslash \mathrm{C}, 3.58498$ $42664,0.1731079472,-0.58918729 \backslash \mathrm{H}, 4.6290560015,0.2314056401,-0.88493589$ $19 \backslash \mathrm{S},-1.4018208475,-1.5981623652,-0.0019989974 \backslash \mathrm{C},-2.8407743157,-1.6904$ $387431,1.1164454541 \backslash \mathrm{H},-3.4397390754,-2.5420528156,0.7830438314 \backslash \mathrm{H},-2.52$ $85067457,-1.8596510317,2.1517464095 \backslash \mathrm{H},-3.4396520659,-0.7786431009,1.05$ $24807841 \backslash \mathrm{S},-1.6058304669,1.4537346691,0.4124901549 \backslash \mathrm{C},-1.6926480643,1.5$ $529707235,-1.4095722981 \backslash \mathrm{H},-2.3798197454,2.3703902428,-1.6429581939 \backslash \mathrm{H},-$ $0.7131453282,1.7672784907,-1.8446896079 \backslash \mathrm{H},-2.0852459047,0.6192369939,-$ $1.8199378508 \backslash \mathrm{H},-0.5812910017,-0.1669076734,1.7485574803 \backslash \backslash$ Version=DEC $-\mathrm{A}$ $\mathrm{XP}-\mathrm{OSF} / 1-\mathrm{G} 98 \mathrm{RevA} .11 .3 \backslash \mathrm{HF}=-1146.5571283 \backslash \mathrm{RMSD}=4.678 \mathrm{e}-09 \backslash \mathrm{RMSF}=3.686 \mathrm{e}-06 \backslash \mathrm{D}$ ipole $=0.0197242,-0.0250547,0.0113779 \backslash \mathrm{PG}=\mathrm{C} 01[\mathrm{X}(\mathrm{C} 9 \mathrm{H} 12 \mathrm{~S} 2)] \backslash \backslash @$

\section{$\mathrm{S}=\mathrm{C}(\mathrm{Ph}) \mathrm{SCH}_{3}$}

$1 \backslash 1 \backslash G I N C-S C 116 \backslash F O p t \backslash R B 3 L Y P \backslash 6-31 G(d) \backslash C 8 H 8 S 2 \backslash M L C 501 \backslash 25-J u 1-2002 \backslash 1 \backslash \backslash \# P$ B3

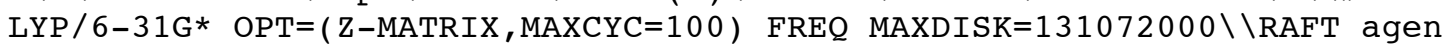
$t \mathrm{~S}=\mathrm{C}(\mathrm{Ph})-\mathrm{S}-\mathrm{CH} 3(\mathrm{Z}=\mathrm{ph}, \mathrm{R}=\mathrm{me}) \backslash \backslash 0,1 \backslash \mathrm{C} \backslash \mathrm{S}, 1, \mathrm{~B} 1 \backslash \mathrm{S}, 1, \mathrm{~B} 2,2, \mathrm{~A} 1 \backslash \mathrm{C}, 3, \mathrm{~B} 3,1, \mathrm{~A} 2,2, \mathrm{D} 1$ , $0 \backslash \mathrm{H}, 4, \mathrm{~B} 4,3, \mathrm{~A} 3,1, \mathrm{D} 2,0 \backslash \mathrm{H}, 4, \mathrm{~B} 5,3, \mathrm{~A} 4,5, \mathrm{D} 3,0 \backslash \mathrm{H}, 4, \mathrm{~B} 6,3, \mathrm{~A} 5,5, \mathrm{D} 4,0 \backslash \mathrm{C}, 1, \mathrm{~B} 7,2, \mathrm{~A}$ $6,3, \mathrm{D} 5,0 \backslash \mathrm{C}, 8, \mathrm{~B} 8,1, \mathrm{~A} 7,2, \mathrm{D} 6,0 \backslash \mathrm{C}, 9, \mathrm{~B} 9,8, \mathrm{~A} 8,1, \mathrm{D} 7,0 \backslash \mathrm{C}, 10, \mathrm{~B} 10,9, \mathrm{~A} 9,8, \mathrm{D} 8,0 \backslash \mathrm{C}$, $11, \mathrm{~B} 11,10, \mathrm{~A} 10,9, \mathrm{D} 9,0 \backslash \mathrm{C}, 12, \mathrm{~B} 12,11, \mathrm{~A} 11,10, \mathrm{D} 10,0 \backslash \mathrm{H}, 13, \mathrm{~B} 13,12, \mathrm{~A} 12,11, \mathrm{D} 11,0$ $\backslash \mathrm{H}, 12, \mathrm{~B} 14,11, \mathrm{~A} 13,13, \mathrm{D} 12,0 \backslash \mathrm{H}, 11, \mathrm{~B} 15,10, \mathrm{~A} 14,12, \mathrm{D} 13,0 \backslash \mathrm{H}, 10, \mathrm{~B} 16,9, \mathrm{~A} 15,11, \mathrm{D}$ $14,0 \backslash \mathrm{H}, 9, \mathrm{~B} 17,8, \mathrm{~A} 16,10, \mathrm{D} 15,0 \backslash \backslash \mathrm{B} 1=1.65390665 \backslash \mathrm{B} 2=1.76783874 \backslash \mathrm{B} 3=1.81735533$ $\backslash \mathrm{B} 4=1.09340644 \backslash \mathrm{B} 5=1.09203382 \backslash \mathrm{B} 6=1.09167768 \backslash \mathrm{B} 7=1.48638437 \backslash \mathrm{B} 8=1.40791986$ $\backslash \mathrm{B} 9=1.3934782 \backslash \mathrm{B} 10=1.3954032 \backslash \mathrm{B} 11=1.39756856 \backslash \mathrm{B} 12=1.39093249 \backslash \mathrm{B} 13=1.084558$ $18 \backslash \mathrm{B} 14=1.08646979 \backslash \mathrm{B} 15=1.08663117 \backslash \mathrm{B} 16=1.08643383 \backslash \mathrm{B} 17=1.08519217 \backslash \mathrm{A} 1=123$. $8831348 \backslash \mathrm{A} 2=103.44785578 \backslash \mathrm{A} 3=105.74311227 \backslash \mathrm{A} 4=110.56367906 \backslash \mathrm{A} 5=110.5050450$ $1 \backslash \mathrm{A} 6=123.43517028 \backslash \mathrm{A} 7=121.7784707 \backslash \mathrm{A} 8=120.59384832 \backslash \mathrm{A} 9=120.24074703 \backslash \mathrm{A} 10=1$ $19.68786961 \backslash \mathrm{A} 11=120.27839448 \backslash \mathrm{A} 12=120.55619114 \backslash \mathrm{A} 13=120.1204252 \backslash \mathrm{A} 14=120$. $14506072 \backslash \mathrm{A} 15=119.5622211 \backslash \mathrm{A} 16=119.91357919 \backslash \mathrm{D} 1=5.07451867 \backslash \mathrm{D} 2=178.7658797$ $5 \backslash D 3=-119.43094734 \backslash D 4=119.41965567 \backslash D 5=181.27901545 \backslash D 6=211.48944504 \backslash D 7=$ $179.74862327 \backslash \mathrm{D} 8=0.5087189 \backslash \mathrm{D} 9=-0.47334322 \backslash \mathrm{D} 10=-0.1123593 \backslash \mathrm{D} 11=180.118421$ $67 \backslash \mathrm{D} 12=179.95923577 \backslash \mathrm{D} 13=180.24754199 \backslash \mathrm{D} 14=180.47358662 \backslash \mathrm{D} 15=182.12059766$

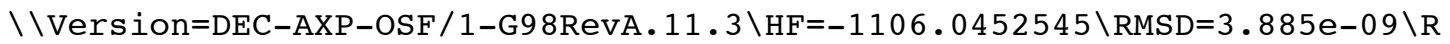
$\mathrm{MSF}=1.915 \mathrm{e}-05 \backslash \mathrm{Dipole}=0.0516049,-0.0290079,-0.8060901 \backslash \mathrm{PG}=\mathrm{C} 01 \quad[\mathrm{X}(\mathrm{C} 8 \mathrm{H} 8 \mathrm{~S} 2)$ ] \\@

\section{$\mathrm{CH}_{3} \mathrm{SC} \cdot\left(\mathrm{CH}_{2} \mathrm{Ph}\right) \mathrm{S}-\mathrm{CH}_{3}(10)$}

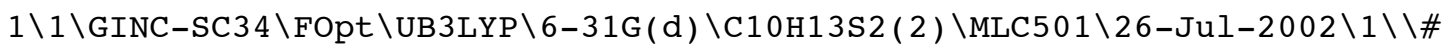
P UB3LYP/6-31G(D) FOPT $=(\mathrm{Z}-\mathrm{MATRIX}, \mathrm{MAXCYC}=100)$ FREQ MAXDISK $=131072000 \backslash \backslash \mathrm{R}$ AFT RAD Me-S-C (MePH)-S-Me ( $\mathrm{Z}=\mathrm{MePH} \mathrm{R}=\mathrm{Me})(1 \mathrm{~b}) \backslash \backslash 0,2 \backslash \mathrm{C} \backslash \mathrm{C}, 1, \mathrm{~B} 1 \backslash \mathrm{H}, 2, \mathrm{~B} 2,1, \mathrm{~A} 1$ $\backslash \mathrm{C}, 2, \mathrm{~B} 3,1, \mathrm{~A} 2,3, \mathrm{D} 1,0 \backslash \mathrm{C}, 4, \mathrm{~B} 4,2, \mathrm{~A} 3,1, \mathrm{D} 2,0 \backslash \mathrm{C}, 5, \mathrm{~B} 5,4, \mathrm{~A} 4,2, \mathrm{D} 3,0 \backslash \mathrm{C}, 6, \mathrm{~B} 6,5, \mathrm{~A} 5$, $4, \mathrm{D} 4,0 \backslash \mathrm{C}, 7, \mathrm{~B} 7,6, \mathrm{~A} 6,5, \mathrm{D} 5,0 \backslash \mathrm{C}, 8, \mathrm{~B} 8,7, \mathrm{~A} 7,6, \mathrm{D} 6,0 \backslash \mathrm{H}, 9, \mathrm{~B} 9,8, \mathrm{~A} 8,7, \mathrm{D} 7,0 \backslash \mathrm{H}, 8, \mathrm{~B} 1$ $0,7, \mathrm{~A} 9,9, \mathrm{D} 8,0 \backslash \mathrm{H}, 7, \mathrm{~B} 11,6, \mathrm{~A} 10,8, \mathrm{D} 9,0 \backslash \mathrm{H}, 6, \mathrm{~B} 12,5, \mathrm{~A} 11,7, \mathrm{D} 10,0 \backslash \mathrm{H}, 5, \mathrm{~B} 13,4, \mathrm{~A} 12$ , $6, \mathrm{D} 11,0 \backslash \mathrm{H}, 2, \mathrm{~B} 14,1, \mathrm{~A} 13,3, \mathrm{D} 12,0 \backslash \mathrm{S}, 1, \mathrm{~B} 15,2, \mathrm{~A} 14,3, \mathrm{D} 13,0 \backslash \mathrm{C}, 16, \mathrm{~B} 16,1, \mathrm{~A} 15,2$, 
$\mathrm{D} 14,0 \backslash \mathrm{H}, 17, \mathrm{~B} 17,16, \mathrm{~A} 16,1, \mathrm{D} 15,0 \backslash \mathrm{H}, 17, \mathrm{~B} 18,16, \mathrm{~A} 17,18, \mathrm{D} 16,0 \backslash \mathrm{H}, 17, \mathrm{~B} 19,16, \mathrm{~A} 18$ , $18, \mathrm{D} 17,0 \backslash \mathrm{S}, 1, \mathrm{~B} 20,2, \mathrm{~A} 19,16, \mathrm{D} 18,0 \backslash \mathrm{C}, 21, \mathrm{~B} 21,1, \mathrm{~A} 20,2$, D19, 0\H , 22 , B22 , $21, \mathrm{~A} 2$ $1,1, \mathrm{D} 20,0 \backslash \mathrm{H}, 22, \mathrm{~B} 23,21, \mathrm{~A} 22,23, \mathrm{D} 21,0 \backslash \mathrm{H}, 22, \mathrm{~B} 24,21, \mathrm{~A} 23,23, \mathrm{D} 22,0 \backslash \backslash \mathrm{B} 1=1.5157$ $8873 \backslash \mathrm{B} 2=1.09765845 \backslash \mathrm{B} 3=1.52389414 \backslash \mathrm{B} 4=1.40058996 \backslash \mathrm{B} 5=1.39681842 \backslash \mathrm{B} 6=1.3955$ $8668 \backslash \mathrm{B} 7=1.39697813 \backslash \mathrm{B} 8=1.39470243 \backslash \mathrm{B} 9=1.08777803 \backslash \mathrm{B} 10=1.08705661 \backslash \mathrm{B} 11=1.08$ $678408 \backslash \mathrm{B} 12=1.08707699 \backslash \mathrm{B} 13=1.08729883 \backslash \mathrm{B} 14=1.09778413 \backslash \mathrm{B} 15=1.75737506 \backslash \mathrm{B} 16$ $=1.8511538 \backslash \mathrm{B} 17=1.09395743 \backslash \mathrm{B} 18=1.09286269 \backslash \mathrm{B} 19=1.09134704 \backslash \mathrm{B} 20=1.75969536$ $\backslash \mathrm{B} 21=1.82647684 \backslash \mathrm{B} 22=1.09192111 \backslash \mathrm{B} 23=1.09332514 \backslash \mathrm{B} 24=1.09120267 \backslash \mathrm{A} 1=108.25$ $525698 \backslash \mathrm{A} 2=113.94938997 \backslash \mathrm{A} 3=120.79198662 \backslash \mathrm{A} 4=120.78731 \backslash \mathrm{A} 5=120.13467307 \backslash \mathrm{A} 6$ $=119.55192775 \backslash \mathrm{A} 7=120.16376976 \backslash \mathrm{A} 8=119.72340424 \backslash \mathrm{A} 9=120.07880369 \backslash \mathrm{A} 10=120$. $24221717 \backslash A 11=119.74087848 \backslash A 12=119.32184346 \backslash A 13=109.03557488 \backslash A 14=120.36$ $439381 \backslash A 15=104.08221211 \backslash A 16=106.22727959 \backslash A 17=110.79627524 \backslash A 18=110.4665$ $5003 \backslash \mathrm{A} 19=115.46042477 \backslash \mathrm{A} 20=104.60546169 \backslash \mathrm{A} 21=110.57101967 \backslash \mathrm{A} 22=105.689005$ $73 \backslash A 23=111.64284706 \backslash D 1=-121.45670912 \backslash D 2=-261.77203284 \backslash D 3=181.36035309 \backslash$ $\mathrm{D} 4=-0.2080065 \backslash \mathrm{D} 5=-0.01087986 \backslash \mathrm{D} 6=0.19371478 \backslash \mathrm{D} 7=-180.12483316 \backslash \mathrm{D} 8=180.168$ $86927 \backslash D 9=180.28334206 \backslash D 10=-179.64263638 \backslash D 11=179.92707274 \backslash D 12=115.75269$ $857 \backslash \mathrm{D} 13=49.66353815 \backslash \mathrm{D} 14=96.41159877 \backslash \mathrm{D} 15=175.79130312 \backslash \mathrm{D} 16=-118.67140069$ $\backslash D 17=119.10764327 \backslash D 18=170.17863131 \backslash D 19=166.2284763 \backslash D 20=-57.73757008 \backslash D 2$ $1=-118.66229821 \backslash \mathrm{D} 22=122.32870879 \backslash \backslash$ Version=DEC-AXP-OSF / 1-G98RevA. $11.3 \backslash \mathrm{H}$ $\mathrm{F}=-1185.2251153 \backslash \mathrm{S} 2=0.755322 \backslash \mathrm{S} 2-1=0 . \backslash \mathrm{S} 2 \mathrm{~A}=0.750022 \backslash \mathrm{RMSD}=2.912 \mathrm{e}-09 \backslash \mathrm{RMSF}=4$ $.358 e-05 \backslash \mathrm{Dipole}=-0.3028549,-0.2300317,-0.3558071 \backslash \mathrm{PG}=\mathrm{C} 01[\mathrm{X}(\mathrm{C} 10 \mathrm{H} 13 \mathrm{~S} 2)] \backslash$ $1 @$

\section{$\mathrm{CH}_{3} \mathrm{SCH}\left(\mathrm{CH}_{2} \mathrm{Ph}\right) \mathrm{S}-\mathrm{CH}_{3}$}

$1 \backslash 1 \backslash G I N C-S C 45 \backslash F O p t \backslash R B 3 L Y P \backslash 6-31 G(d) \backslash C 10 H 14 S 2 \backslash M L C 501 \backslash 16-J a n-2003 \backslash 0 \backslash \backslash \# N B$

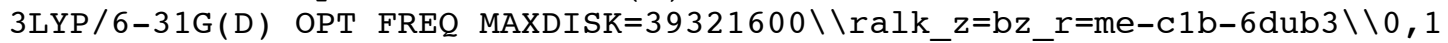
$\backslash \mathrm{C},-0.6173236939,0.8705443939,1.2131640708 \backslash \mathrm{C}, \overline{0} .923 \overline{7} 250599,0.7648487152$ $, 1.0849107713 \backslash \mathrm{H}, 1.2967000529,1.7932607337,1.0238423875 \backslash \mathrm{C}, 1.4867514685$, $-0.0328239565,-0.0734777501 \backslash C, 1.9305433816,0.6176027169,-1.2335000474 \backslash$ C, $2.4531446505,-0.1058521511,-2.3068796554 \backslash \mathrm{C}, 2.5473904727,-1.496865814$ $9,-2.233928628 \backslash \mathrm{C}, 2.1233471121,-2.1556306262,-1.0785833688 \backslash \mathrm{C}, 1.60288691$ $13,-1.4286929396,-0.0070365185 \backslash \mathrm{H}, 1.2883503964,-1.9503596197,0.89333675$ $28 \backslash \mathrm{H}, 2.2028416753,-3.2372772459,-1.0074592618 \backslash \mathrm{H}, 2.9565146813,-2.062006$ $6475,-3.0672017016 \backslash \mathrm{H}, 2.792301443,0.4181302663,-3.1968501978 \backslash \mathrm{H}, 1.860668$ $751,1.7009148982,-1.2946961883 \backslash \mathrm{H}, 1.2923834981,0.3471511213,2.031044895$ $7 \backslash \mathrm{S},-1.4631255803,1.7953396077,-0.154444498 \backslash \mathrm{C},-1.6190494167,0.57428668$ $07,-1.5076286646 \backslash \mathrm{H},-2.3490067503,0.9950847255,-2.204695011 \backslash \mathrm{H},-1.999105$ $8567,-0.3722543252,-1.115376466 \backslash \mathrm{H},-0.6720362494,0.4090748799,-2.022664$ $821 \backslash \mathrm{S},-1.357679492,-0.7650043445,1.6345277873 \backslash \mathrm{C},-2.9698366519,-0.21865$ $98111,2.2962358868 \backslash \mathrm{H},-2.8455526925,0.3638737345,3.2142898163 \backslash \mathrm{H},-3.5410$ $960903,-1.1226708724,2.5227632944 \backslash \mathrm{H},-3.5083692885,0.3740231519,1.55239$ $74561 \backslash \mathrm{H},-0.8111881781,1.5251477451,2.0702798393 \backslash \backslash$ Version=DEC-AXP-OSF / 1 -G98RevA. 11.3 \HF=-1185.8720897\RMSD=4 .156e-09 \RMSF=7 .912e-06 $\backslash \mathrm{Dipole}=-0$ $.0376313,-0.1232866,0.0534949 \backslash \mathrm{PG}=\mathrm{C} 01 \quad[\mathrm{X}(\mathrm{C} 10 \mathrm{H} 14 \mathrm{~S} 2)] \backslash \backslash @$

\section{$\mathrm{S}=\mathrm{C}\left(\mathrm{CH}_{2} \mathrm{Ph}\right) \mathrm{SCH}_{3}$}

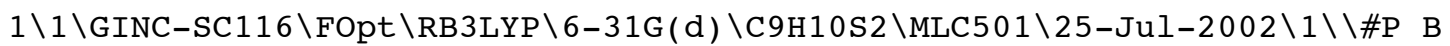
3LYP/6-31G* OPT $=(Z-M A T R I X, M A X C Y C=100)$ FREQ MAXDISK=131072000\\RAFT Age nt $\mathrm{S}=\mathrm{C}(\mathrm{MePH})-\mathrm{SMe}(\mathrm{Z}=\mathrm{MePh}, \mathrm{R}=\mathrm{Me}) \mathrm{C} 1 \backslash \backslash 0,1 \backslash \mathrm{C} \backslash \mathrm{S}, 1, \mathrm{~B} 1 \backslash \mathrm{C}, 1, \mathrm{~B} 2,2, \mathrm{~A} 1 \backslash \mathrm{H}, 3, \mathrm{~B} 3,1$, $\mathrm{A} 2,2, \mathrm{D} 1,0 \backslash \mathrm{H}, 3, \mathrm{~B} 4,1, \mathrm{~A} 3,4, \mathrm{D} 2,0 \backslash \mathrm{C}, 3, \mathrm{~B} 5,1, \mathrm{~A} 4,4, \mathrm{D} 3,0 \backslash \mathrm{C}, 6, \mathrm{~B} 6,3, \mathrm{~A} 5,1, \mathrm{D} 4,0 \backslash \mathrm{C}, 7$ , $\mathrm{B} 7,6, \mathrm{~A} 6,3, \mathrm{D} 5,0 \backslash \mathrm{C}, 8, \mathrm{~B} 8,7, \mathrm{~A} 7,6, \mathrm{D} 6,0 \backslash \mathrm{C}, 9, \mathrm{~B} 9,8, \mathrm{~A} 8,7, \mathrm{D} 7,0 \backslash \mathrm{C}, 10, \mathrm{~B} 10,9, \mathrm{~A} 9,8$, $\mathrm{D} 8,0 \backslash \mathrm{H}, 11, \mathrm{~B} 11,10, \mathrm{~A} 10,9, \mathrm{D} 9,0 \backslash \mathrm{H}, 10, \mathrm{~B} 12,9, \mathrm{~A} 11,11, \mathrm{D} 10,0 \backslash \mathrm{H}, 9, \mathrm{~B} 13,8, \mathrm{~A} 12,10, \mathrm{D}$ $11,0 \backslash \mathrm{H}, 8, \mathrm{~B} 14,7, \mathrm{~A} 13,9, \mathrm{D} 12,0 \backslash \mathrm{H}, 7, \mathrm{~B} 15,6, \mathrm{~A} 14,8, \mathrm{D} 13,0 \backslash \mathrm{S}, 1, \mathrm{~B} 16,2, \mathrm{~A} 15,3, \mathrm{D} 14,0$ $\backslash \mathrm{C}, 17, \mathrm{~B} 17,1, \mathrm{~A} 16,2, \mathrm{D} 15,0 \backslash \mathrm{H}, 18, \mathrm{~B} 18,16, \mathrm{~A} 17,1, \mathrm{D} 16,0 \backslash \mathrm{H}, 18, \mathrm{~B} 19,16, \mathrm{~A} 18,1, \mathrm{D} 17$, $0 \backslash \mathrm{H}, 18, \mathrm{~B} 20,16, \mathrm{~A} 19,1, \mathrm{D} 18,0 \backslash \backslash \mathrm{B} 1=1.64564466 \backslash \mathrm{B} 2=1.52863415 \backslash \mathrm{B} 3=1.09674628 \backslash \mathrm{B}$ $4=1.09353236 \backslash B 5=1.52343747 \backslash B 6=1.39968158 \backslash B 7=1.39594042 \backslash B 8=1.39529816 \backslash B$ $9=1.39667801 \backslash \mathrm{B} 10=1.3946779 \backslash \mathrm{B} 11=1.08659727 \backslash \mathrm{B} 12=1.08682646 \backslash \mathrm{B} 13=1.0867289$ 
$4 \backslash \mathrm{B} 14=1.08683319 \backslash \mathrm{B} 15=1.08756909 \backslash \mathrm{B} 16=1.7547672 \backslash \mathrm{B} 17=1.81824902 \backslash \mathrm{B} 18=1.093$ $09254 \backslash \mathrm{B} 19=1.09194447 \backslash \mathrm{B} 20=1.0919036 \backslash \mathrm{A} 1=123.05916516 \backslash \mathrm{A} 2=109.80486391 \backslash \mathrm{A} 3=$ $107.35736989 \backslash A 4=112.35357201 \backslash A 5=120.55086294 \backslash A 6=120.654591 \backslash A 7=120.0667$ $1159 \backslash A 8=119.64159031 \backslash A 9=120.22881755 \backslash A 10=120.05599167 \backslash A 11=120.09413459$ $\backslash \mathrm{A} 12=120.18319366 \backslash \mathrm{A} 13=119.73589867 \backslash \mathrm{A} 14=119.61324586 \backslash \mathrm{A} 15=125.66992548 \backslash \mathrm{A}$ $16=103.79835693 \backslash A 17=105.33482935 \backslash A 18=93.82431614 \backslash A 19=126.14621 \backslash D 1=150$. $7138342 \backslash D 2=-116.89084223 \backslash D 3=121.89888903 \backslash D 4=-100.14574145 \backslash D 5=179.75805$ $35 \backslash D 6=-0.07990219 \backslash D 7=0.06511684 \backslash D 8=-0.01890592 \backslash D 9=180.33270637 \backslash D 10=179$ $.82110496 \backslash \mathrm{D} 11=-180.19616308 \backslash \mathrm{D} 12=179.8418732 \backslash \mathrm{D} 13=179.81914239 \backslash \mathrm{D} 14=-178$. $68759297 \backslash \mathrm{D} 15=2.34439814 \backslash \mathrm{D} 16=197.73877987 \backslash \mathrm{D} 17=85.44790483 \backslash \mathrm{D} 18=-31.96744$

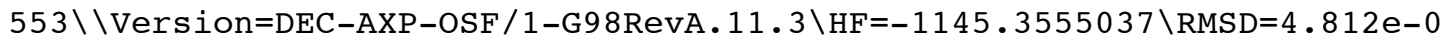
$9 \backslash \mathrm{RMSF}=1.589 \mathrm{e}-05 \backslash \mathrm{Dipole}=-0.341854,0.1539887,-0.6355984 \backslash \mathrm{PG}=\mathrm{C} 01] \mathrm{X}(\mathrm{C} 9 \mathrm{H} 10$ $\mathrm{S} 2) \mathrm{J} \backslash \backslash @$

\section{$\mathrm{CH}_{3} \mathrm{SC} \cdot\left(\right.$ naphthyl) $\mathrm{SCH}_{3}(11)$}

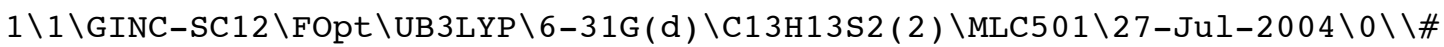

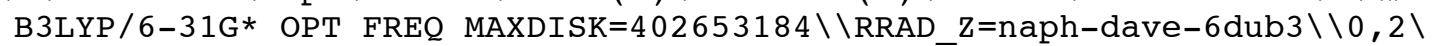
C $, 0.343850159,-1.7487899687,-0.86824 \backslash C,-0.7940 \overline{8} 97714,-2.5148100722,-1$. $16862 \backslash C,-2.0529298139,-2.0473101866,-0.859 \backslash C,-2.2095399273,-0.79964020$ $09,-0.19851 \backslash \mathrm{C}, 0.2614300445,-0.4894399762,-0.25296 \backslash \mathrm{C},-1.0562599991,-0.0$ $09810096,0.13347 \backslash \mathrm{C},-3.5013999695,-0.3354903183,0.16899 \backslash \mathrm{C},-1.2665801073$ $, 1.1805098848,0.87891 \backslash \mathrm{C},-3.6675300764,0.8402596666,0.86432 \backslash \mathrm{C},-2.533940$ $1452,1.5968697696,1.23325 \backslash \mathrm{C}, 1.4894899773,0.2492501354,-0.02396 \backslash \mathrm{S}, 3.018$ $1300501,-0.5507697256,0.35789 \backslash \mathrm{S}, 1.7488598212,1.966630159,-0.32114 \backslash \mathrm{C}, 2$. $5603601535,-1.6881697672,1.72193 \backslash \mathrm{C}, 0.6445797868,2.3454800586,-1.73332 \backslash$ $\mathrm{H}, 1.8346302214,-2.4348098332,1.39273 \backslash \mathrm{H}, 3.485290199,-2.1885496831,2.021$ $71 \backslash \mathrm{H}, 2.1602001024,-1.1261298036,2.56888 \backslash \mathrm{H},-0.4055002131,2.3434299631$, $1.43337 \backslash \mathrm{H}, 0.9248896957,3.3469100841,-2.07139 \backslash \mathrm{H}, 0.8060398518,1.62974007$ $33,-2.54252 \backslash \mathrm{H},-0.4099401608,1.7685199627,1.18709 \backslash \mathrm{H},-2.6577302283,2.511$ $1597584,1.80751 \backslash \mathrm{H},-4.6622601072,1.1789795761,1.14112 \backslash \mathrm{H},-4.3628399144,-$ $0.9412303967,-0.10281 \backslash \mathrm{H},-2.939069761,-2.6287202672,-1.10053 \backslash \mathrm{H},-0.67216$ $96843,-3.4718400611,-1.66914 \backslash \mathrm{H}, 1.3220401923,-2.1145598798,-1.16481 \backslash \backslash \mathrm{Ve}$ rsion=DEC-AXP-OSF $/ 1-G 03 R e v B .03 \backslash$ State $=2-A \backslash H F=-1299.5619478 \backslash S 2=0.771347 \backslash$ $\mathrm{S} 2-1=0 . \backslash \mathrm{S} 2 \mathrm{~A}=0.750301 \backslash \mathrm{RMSD}=6.132 \mathrm{e}-09 \backslash \mathrm{RMSF}=3.161 \mathrm{e}-06 \backslash \mathrm{Dipole}=-0.6674939,-$ $0.2830366,-0.0542894 \backslash \mathrm{PG}=\mathrm{C} 01 \quad[\mathrm{X}(\mathrm{C} 13 \mathrm{H} 13 \mathrm{~S} 2)] \backslash \backslash @$

\section{$\mathrm{CH}_{3} \mathrm{SCH}$ (naphthyl)SCH}

$1 \backslash 1 \backslash$ GINC-SC35 $\backslash$ FOpt $\backslash R B 3 L Y P \backslash 6-31 G(d) \backslash C 13 H 14 S 2 \backslash M L C 501 \backslash 27-J u l-2004 \backslash 0 \backslash \backslash \#$ B3

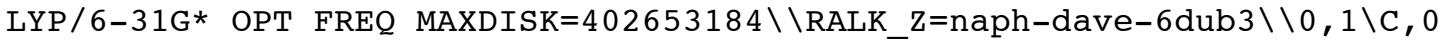
$.3429103148,1.4801899271,0.80982 \backslash \mathrm{C},-0.73351 \overline{9} 5225,2.245200156,1.31517 \backslash \mathrm{C}$ $,-2.0283196047,1.8590304313,1.07136 \backslash \mathrm{C},-2.2996398552,0.680910489,0.3258$ $7 \backslash C, 0.1376500699,0.3284799707,0.07225 \backslash C,-1.2110300239,-0.1124697425,-0$ $.17428 \backslash \mathrm{C},-3.6368299419,0.2732207734,0.07233 \backslash \mathrm{C},-1.5344702766,-1.3007496$ $737,-0.88768 \backslash \mathrm{C},-3.908740186,-0.8747591688,-0.6345 \backslash \mathrm{C},-2.8427403551,-1.6$ $696393955,-1.11356 \backslash \mathrm{C}, 1.3122198991,-0.4742302791,-0.45841 \backslash \mathrm{S}, 2.846860103$ $, 0.4844093946,-0.85489 \backslash S, 1.784539596,-1.8996803795,0.62687 \backslash C, 2.2040603$ $567,1.6773095313,-2.08556 \backslash \mathrm{C}, 2.0863997681,-1.0905904437,2.23674 \backslash \mathrm{H}, 1.468$ $9405009,2.3552696876,-1.64483 \backslash \mathrm{H}, 3.0651404796,2.2553793482,-2.4325 \backslash \mathrm{H}, 1$. $7618102465,1.1592796253,-2.94219 \backslash \mathrm{H}, 1.1906898764,-0.5811702532,2.59979 \backslash$ $\mathrm{H}, 2.3451295965,-1.8972004987,2.92815 \backslash \mathrm{H}, 2.9216699174,-0.3886206213,2.18$ $057 \backslash \mathrm{H},-0.7435304144,-1.9487898419,-1.24734 \backslash \mathrm{H},-3.0562305496,-2.58445935$ $01,-1.65972 \backslash \mathrm{H},-4.93618025,-1.1756889503,-0.81978 \backslash \mathrm{H},-4.4464598107,0.890$ $2509456,0.45515 \backslash \mathrm{H},-2.8638594798,2.446310609,1.44468 \backslash \mathrm{H},-0.5250193312,3$. $1448101117,1.88781 \backslash \mathrm{H}, 1.3610803856,1.8130697105,0.98728 \backslash \mathrm{H}, 1.0267997926$, $-0.9754502184,-1.38609 \backslash \backslash$ Version=DEC-AXP-OSF $/ 1-G 03$ RevB . 03 $\backslash$ State $=1-\mathrm{A} \backslash \mathrm{HF}=$ $-1300.1987175 \backslash \mathrm{RMSD}=3.018 \mathrm{e}-09 \backslash \mathrm{RMSF}=5.664 \mathrm{e}-06 \backslash \mathrm{Dipole}=-0.7113722,0.640756$ $8,0.0278507 \backslash \mathrm{PG}=\mathrm{C} 01 \quad[\mathrm{X}(\mathrm{C} 13 \mathrm{H} 14 \mathrm{~S} 2)] \backslash \backslash \mathrm{Q}$ 


\section{$\mathrm{CH}_{3} \mathrm{SCH}$ (naphthyl)=S}

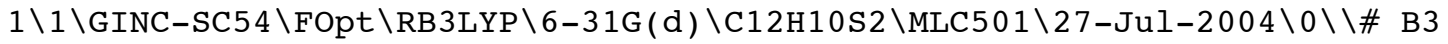

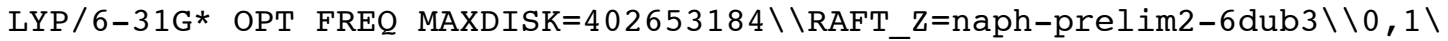
$\mathrm{S},-1.3176849754,1.6737176585,-1.5524446266 \backslash \overline{\mathrm{C}},-0.2009937358,1.438309753$ $4,-0.3595824768 \backslash \mathrm{S}, 0.2740769273,2.6480988068,0.8298250319 \backslash \mathrm{C},-0.67357585$ $74,4.1224715777,0.3431500034 \backslash \mathrm{H},-0.4106710102,4.4286183672,-0.671738077$ $3 \backslash \mathrm{H},-0.4029386599,4.9042859452,1.0579069976 \backslash \mathrm{H},-1.7454419648,3.91937227$ $21,0.3911407128 \backslash \mathrm{C}, 0.5720343078,0.1760626079,-0.1699894095 \backslash \mathrm{C}, 1.95550227$ $92,0.241356741,-0.2535616377 \backslash \mathrm{C},-0.068403214,-1.0901710315,0.0732982734$ $\backslash \mathrm{C}, 2.7553831675,-0.9170429272,-0.1508974039 \backslash \mathrm{C}, 2.1682694836,-2.14047870$ $94,0.0640278079 \backslash \mathrm{C}, 0.7587833876,-2.2575264346,0.1924411878 \backslash \mathrm{C}, 0.15287213$ $57,-3.5172428725,0.4478622577 \backslash \mathrm{C},-1.2092665558,-3.6310698062,0.59745296$ $\backslash \mathrm{C},-1.4704186791,-1.2443776571,0.2467639923 \backslash \mathrm{C},-2.0241863606,-2.4794356$ $632,0.5019496693 \backslash \mathrm{H}, 2.4306166215,1.1994383427,-0.4388929989 \backslash \mathrm{H}, 3.8338029$ $014,-0.830633207,-0.2472790745 \backslash \mathrm{H},-2.1124085748,-0.3752773959,0.1740860$ $224 \backslash \mathrm{H},-3.0993118832,-2.5689544227,0.631209707 \backslash \mathrm{H},-1.6604563749,-4.59969$ $80693,0.7941883627 \backslash \mathrm{H}, 0.7932196361,-4.3923389191,0.5295042721 \backslash \mathrm{H}, 2.77531$ $59248,-3.0390098275,0.1442962488 \backslash \backslash$ Version=DEC-AXP-OSF / 1-G03RevB.03 \Sta $t e=1-A \backslash H F=-1259.6829896 \backslash R M S D=5.348 e-09 \backslash R M S F=1.703 e-06 \backslash \mathrm{Dipole}=0.4154548$ $, 0.0290482,0.5913311 \backslash \mathrm{PG}=\mathrm{C} 01[\mathrm{X}(\mathrm{C} 12 \mathrm{H} 10 \mathrm{~S} 2)] \backslash \backslash @$

\section{$\mathrm{CH}_{3} \mathrm{SC} \cdot\left(\mathrm{OCH}_{3}\right) \mathrm{SCH}_{3}(12)$}

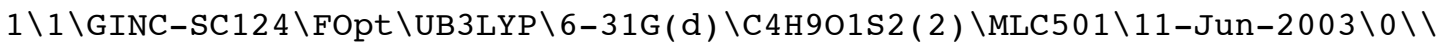
\# B3LYP/6-31G* OPT $=($ MAXCYC $=100)$ FREQ MAXDISK $=13107200$ GEOM=CHECK GUESS $=\mathrm{READ} \backslash \backslash \mathrm{rrad} z=0 m e \_r=m e-a 1 \mathrm{~b} 1 \mathrm{c} 1 \mathrm{~d} 3 \mathrm{e} 2 \mathrm{f} 1-6 \mathrm{dub} \backslash \backslash \backslash 0,2 \backslash \mathrm{S},-1.3410354108,-1.1021$ $645587,0.00 \overline{8} 37807 \overline{2} 2 \backslash \mathrm{C},-0.0668314555,0.0846927765,-0.2625382649 \backslash \mathrm{S}, 1.525$ $0665723,-0.5327357432,-0.7113926887 \backslash \mathrm{C}, 2.3426793192,-0.7855867589,0.929$ $7015005 \backslash \mathrm{C},-2.8220656021,-0.0301869566,0.1187230656 \backslash 0,-0.1829188202,1.2$ $334385003,0.4787862469 \backslash \mathrm{C}, 0.3588901181,2.4188570239,-0.1145681207 \backslash \mathrm{H},-2$. $9779210372,0.5082852285,-0.8196451416 \backslash \mathrm{H},-3.670068575,-0.6955390127,0.2$ $998754487 \backslash \mathrm{H},-2.72432788,0.675718492,0.9443720255 \backslash \mathrm{H}, 2.2450048802,0.1147$ $964264,1.5398219577 \backslash \mathrm{H}, 3.400214885,-0.9780596806,0.7296543233 \backslash \mathrm{H}, 1.90496$ $92578,-1.6399132978,1.4510056405 \backslash \mathrm{H}, 0.1716693923,3.2223284691,0.6006696$ $643 \backslash \mathrm{H}, 1.4348670988,2.3181216146,-0.2907133132 \backslash \mathrm{H},-0.1415903241,2.638502$ $0801,-1.0650057993 \backslash \backslash$ Version=DEC-AXP-OSF $/ 1-$ G03RevB . 03 $\backslash$ State $=2-A \backslash H F=-102$ $9.3786222 \backslash \mathrm{S} 2=0.754676 \backslash \mathrm{S} 2-1=0 . \backslash \mathrm{S} 2 \mathrm{~A}=0.750016 \backslash \mathrm{RMSD}=3.068 \mathrm{e}-09 \backslash \mathrm{RMSF}=1.311 \mathrm{e}-$ $06 \backslash \mathrm{Dipole}=-0.1181936,0.8010544,0.3889717 \backslash \mathrm{PG}=\mathrm{C} 01 \quad[\mathrm{X}(\mathrm{C} 4 \mathrm{H} 901 \mathrm{~S} 2)] \backslash \backslash @$

\section{$\mathrm{CH}_{3} \mathrm{SCH}\left(\mathrm{OCH}_{3}\right) \mathrm{SCH}_{3}$}

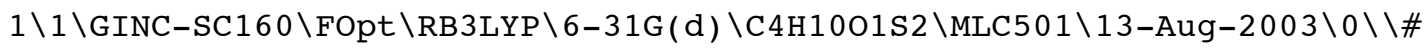
B3LYP $/ 6-31 \mathrm{G} *$ OPT $=($ MAXCYC $=100, \mathrm{TIGHT})$ FREQ GEOM=CHECK GUESS=READ MAXDISK $=39321600$ INT (GRID=ULTRAFINE) \\ralk_z=ome_r=me-a1b1c1d3e2f $1-6$ dub3 $\backslash \backslash 0,1$ $\backslash \mathrm{S},-1.2638080102,-1.0946066938,-0.0 \overline{3} 88623 \overline{5} 7 \backslash \mathrm{C},-0.0661509511,0.22262651$ $51,-0.4773860228 \backslash \mathrm{S}, 1.6208062742,-0.506946341,-0.6859074301 \backslash \mathrm{C}, 1.9762100$ $951,-1.0009324492,1.0416521861 \backslash \mathrm{C},-2.7837706451,-0.0914568117,0.1427433$ $606 \backslash 0,-0.1350263577,1.2360458069,0.4876130868 \backslash \mathrm{C}, 0.4025768371,2.4839329$ $534,0.0588080284 \backslash \mathrm{H},-3.067093543,0.3769686088,-0.804977898 \backslash \mathrm{H},-3.5760666$ $241,-0.7816445842,0.4449633038 \backslash \mathrm{H},-2.649622786,0.6701274942,0.912327733$ $8 \backslash \mathrm{H}, 1.6788946359,-0.1976058747,1.7188468452 \backslash \mathrm{H}, 3.0563328618,-1.15320241$ $87,1.1078188609 \backslash \mathrm{H}, 1.458985461,-1.9239787154,1.3096252981 \backslash \mathrm{H}, 0.253256020$ $9,3.1821978333,0.8850644167 \backslash \mathrm{H}, 1.4725885627,2.4014309461,-0.1672236522 \backslash$ $\mathrm{H},-0.1241541813,2.8566842465,-0.8321509639 \backslash \mathrm{H},-0.3080737866,0.620483318$ $7,-1.473787359 \backslash \backslash$ Version=DEC-AXP-OSF / 1-G03RevB.03 $\backslash$ State $=1-\mathrm{A} \backslash \mathrm{HF}=-1030.02$ $74547 \backslash \mathrm{RMSD}=3.600 \mathrm{e}-09 \backslash \mathrm{RMSF}=3.094 \mathrm{e}-07 \backslash \mathrm{Dipole}=-0.2244423,0.8750638,0.1944$ $09 \backslash \mathrm{PG}=\mathrm{C} 01 \quad[\mathrm{X}(\mathrm{C} 4 \mathrm{H} 1001 \mathrm{~S} 2)] \backslash \backslash \mathrm{Q}$ 


\section{$\mathrm{S}=\mathrm{C}\left(\mathrm{OCH}_{3}\right) \mathrm{SCH}_{3}$}

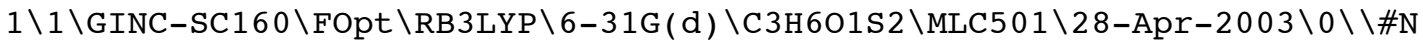
B3LYP $/ 6-31 \mathrm{G}(\mathrm{D}) \quad \mathrm{OPT}=(\mathrm{TIGHT}, \mathrm{MAXCYC}=100) \quad \mathrm{FREQ}$ MAXDISK $=131072000$ GEOM=CHEC $K$ GUESS $=$ READ $\backslash$ RAFT $(\mathrm{Z}=\mathrm{OMe} \mathrm{R}=\mathrm{CH} 3)$ lowest $\mathrm{a} 1 \mathrm{~b} 1$ in $\mathrm{Cs}$ symm $\backslash \backslash 0,1 \backslash \mathrm{C}, 0.374209$ $3441,-2.4921458868,0 . \backslash S, 1.301426637,-0.9260065359,0 . \backslash C,-0.0000019362,0$ $.2785978098,0 . \backslash S,-1.6256039629,-0.0059529119,0 . \backslash 0,0.5982011851,1.47426$ $53379,0 . \backslash \mathrm{C},-0.2142814517,2.6603482395,0 . \backslash \mathrm{H}, 1.1323627109,-3.280322659,0$ $. \backslash \mathrm{H},-0.2490760809,-2.5725262915,0.8922739391 \backslash \mathrm{H},-0.2490760809,-2.572526$ $2915,-0.8922739391 \backslash \mathrm{H}, 0.4987378961,3.485149722,0 . \backslash \mathrm{H},-0.8456382241,2.688$ $326503,-0.8916364756 \backslash \mathrm{H},-0.8456382241,2.688326503,0.8916364756 \backslash \backslash$ Version $=\mathrm{DEC}-\mathrm{AXP}-\mathrm{OSF} / 1-\mathrm{G} 98 \mathrm{RevA} \cdot 11.3 \backslash \mathrm{State}=1-\mathrm{A}^{\prime} \backslash \mathrm{HF}=-989.5242355 \backslash \mathrm{RMSD}=5.328 \mathrm{e}-09 \backslash$ $\mathrm{RMSF}=3.625 \mathrm{e}-06 \backslash \mathrm{Dipole}=0.2790653,0.1622872,0 . \backslash \mathrm{PG}=\mathrm{CS} \quad[\mathrm{SG}(\mathrm{C} 3 \mathrm{H} 2 \mathrm{O} 1 \mathrm{~S} 2), \mathrm{X}(\mathrm{H} 4)$ ]$\backslash \backslash @$

\section{$\mathrm{CH}_{3} \mathrm{SC} \cdot\left(\mathrm{OCH}_{2} \mathrm{CH}_{3}\right) \mathrm{SCH}_{3}(13)$}

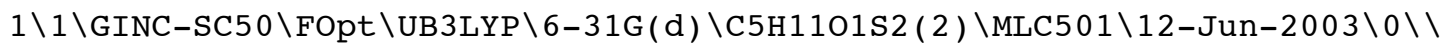
\# B3LYP/6-31G* OPT $=($ MAXCYC=100) FREQ MAXDISK $=134217728$ GEOM=CHECK GUES $S=R E A D \backslash \backslash r r a d \_z=o e t \_r=m e-a 1 b 1 c 1 d 3 e 2 f 1-6$ dub3 $\backslash \backslash 0,2 \backslash S, 1.7423907621,-0.9654$ $242546,0.1280802564 \backslash \mathrm{C}, 0.3393190694,0.0076784401,-0.3116956685 \backslash \mathrm{S}, 0.6303$ $178003,1.7122689272,-0.6718428413 \backslash \mathrm{C}, 0.4396160625,2.5167306849,0.983741$ $8624 \backslash \mathrm{C}, 1.0419958484,-2.6568847766,0.081707205 \backslash 0,-0.8417886969,-0.39199$ $54224,0.2566619351 \backslash \mathrm{C},-2.0409053693,-0.1258027406,-0.4989653844 \backslash \mathrm{H}, 0.698$ $0673109,-2.9039347333,-0.9259399649 \backslash \mathrm{H}, 1.8526385793,-3.3333648574,0.363$ $9360802 \backslash \mathrm{H}, 0.2195155158,-2.7506488733,0.7920901202 \backslash \mathrm{H},-0.4988810008,2.20$ $4729172,1.4468746738 \backslash \mathrm{H}, 0.4197718259,3.5961627799,0.8106289169 \backslash \mathrm{H}, 1.2795$ $067643,2.2635964141,1.6346466759 \backslash C,-3.2099223896,-0.6971972568,0.28261$ $14787 \backslash \mathrm{H},-1.9487825653,-0.5924504824,-1.4882299133 \backslash \mathrm{H},-2.1466192075,0.95$ $52698805,-0.646297879 \backslash \mathrm{H},-4.145906282,-0.5121722605,-0.255684689 \backslash \mathrm{H},-3.0$ $976073558,-1.7774777593,0.420113456 \backslash \mathrm{H},-3.2813503359,-0.2304067694,1.27$ $03714428 \backslash \backslash$ Version=DEC-AXP-OSF $/ 1-G 03$ RevB.03 $\backslash$ State $=2-A \backslash H F=-1068.6978609 \backslash$ $\mathrm{S} 2=0.754682 \backslash \mathrm{S} 2-1=0 . \backslash \mathrm{S} 2 \mathrm{~A}=0.750016 \backslash \mathrm{RMSD}=4.362 \mathrm{e}-09 \backslash \mathrm{RMSF}=4.589 \mathrm{e}-05 \backslash \mathrm{Dipole}=$ $-0.8687421,-0.3333734,0.2996148 \backslash \mathrm{PG}=\mathrm{C} 01 \quad[\mathrm{X}(\mathrm{C} 5 \mathrm{H} 11 \mathrm{O} 1 \mathrm{~S} 2)] \backslash \backslash @$

\section{$\mathrm{CH}_{3} \mathrm{SCH}\left(\mathrm{OCH}_{2} \mathrm{CH}_{3}\right) \mathrm{SCH}_{3}$}

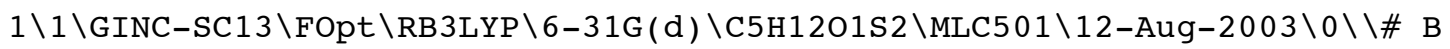
$3 \mathrm{LYP} / 6-31 \mathrm{G} *$ OPT $=($ MAXCYC $=100$, TIGHT $)$ FREQ MAXDISK $=52428800 \backslash \backslash \mathrm{ralk} z=$ oet_r $=$ ene-a1b1c1d3e2f $1-6$ dub3 $\backslash \backslash 0,1 \backslash S, 1.7350532008,-0.8457180216,0.198 \overline{7} 28565 \overline{5} \backslash$ $\mathrm{C}, 0.2760627271,-0.0324436119,-0.5609742453 \backslash \mathrm{S}, 0.5683536315,1.7911469295$ $,-0.6755397055 \backslash \mathrm{C}, 0.5625629974,2.2322542858,1.1021329212 \backslash \mathrm{C}, 1.1505721514$ $,-2.5795837844,0.2281090989 \backslash 0,-0.8689397851,-0.3838474539,0.1631698645$ $\backslash \mathrm{C},-2.1003979789,-0.2126540763,-0.5502828295 \backslash \mathrm{H}, 0.9950240852,-2.9648260$ $008,-0.7844760863 \backslash \mathrm{H}, 1.938518026,-3.1645204188,0.7101910097 \backslash \mathrm{H}, 0.2291044$ $198,-2.6615619388,0.8065435561 \backslash \mathrm{H},-0.2560054802,1.7113196387,1.60313207$ $85 \backslash \mathrm{H}, 0.3967325693,3.3109979203,1.1575695924 \backslash \mathrm{H}, 1.5108971941,1.982303522$ $5,1.5813527577 \backslash \mathrm{C},-3.2313386966,-0.6726756556,0.3525702705 \backslash \mathrm{H},-2.0697663$ $819,-0.8063556975,-1.4776439769 \backslash \mathrm{H},-2.2203393233,0.8418006672,-0.831633$ $9732 \backslash \mathrm{H},-4.1924423638,-0.5611587779,-0.1610822814 \backslash \mathrm{H},-3.1057966298,-1.72$ $45589306,0.6289735018 \backslash \mathrm{H},-3.25881493,-0.0769365926,1.270496072 \backslash \mathrm{H}, 0.1851$ $305764,-0.3519692325,-1.6091342205 \backslash \backslash$ Version=DEC-AXP-OSF / 1-G03RevB.03\S tate $=1-\mathrm{A} \backslash \mathrm{HF}=-1069.3466909 \backslash \mathrm{RMSD}=3.822 \mathrm{e}-09 \backslash \mathrm{RMSF}=4.908 \mathrm{e}-06 \backslash \mathrm{Dipole}=-0.8658$ $269,-0.4759374,0.0482893 \backslash P G=C 01 \quad[X(\mathrm{C} 5 \mathrm{H} 12 \mathrm{O} 1 \mathrm{~S} 2)] \backslash \backslash @$

\section{$\mathrm{S}=\mathrm{C}\left(\mathrm{OCH}_{2} \mathrm{CH}_{3}\right) \mathrm{SCH}_{3}$}

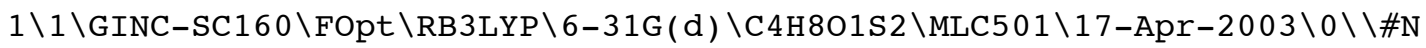
B3LYP $/ 6-31 \mathrm{G}(\mathrm{D}) \quad \mathrm{OPT}=(\mathrm{TIGHT}, \mathrm{MAXCYC}=100) \quad$ FREQ MAXDISK $=39321600 \backslash \backslash \mathrm{RAFT}(\mathrm{Z}=\mathrm{Oe}$ $t \mathrm{R}=\mathrm{Me})$ alb1 $\mathrm{Cs} \backslash \backslash 0,1 \backslash 0,-1.1437055573,-0.0071493756,0 . \backslash \mathrm{C}, 0.1908056904,0$ $.0159885534,0 . \backslash S, 1.1547744866,1.3566881401,0 . \backslash S, 0.7173978682,-1.679486$ 
$5418,0 . \backslash \mathrm{C},-1.881632827,1.2406966192,0 . \backslash \mathrm{C}, 2.530332313,-1.5196200975,0 . \backslash$ $\mathrm{H}, 2.9139622205,-2.5438259958,0 . \backslash \mathrm{H}, 2.8722378969,-0.9919429029,-0.892046$ $9855 \backslash \mathrm{H}, 2.8722378969,-0.9919429029,0.8920469855 \backslash \mathrm{C},-3.3553265843,0.88286$ $80162,0 . \backslash \mathrm{H},-1.5898648833,1.8145031619,-0.8849261225 \backslash \mathrm{H},-1.5898648833,1$. $8145031619,0.8849261225 \backslash \mathrm{H},-3.9527537929,1.8009113963,0 . \backslash \mathrm{H},-3.618069613$ $, 0.3000824834,-0.8882588427 \backslash \mathrm{H},-3.618069613,0.3000824834,0.8882588427 \backslash \backslash$ Version=DEC-AXP-OSF $/ 1-G 98 R e v A .11 .3 \backslash$ State $=1-A^{\prime} \backslash \mathrm{HF}=-1028.8435755 \backslash \mathrm{RMSD}=6$. $745 \mathrm{e}-09 \backslash \mathrm{RMSF}=2.655 \mathrm{e}-06 \backslash \mathrm{Dipole}=-0.403286,-0.187352,0 . \backslash \mathrm{PG}=\mathrm{CS} \quad[\mathrm{SG}(\mathrm{C} 4 \mathrm{H} 2 \mathrm{O} 1 \mathrm{~S}$ $2), \mathrm{X}(\mathrm{H} 6) \mathrm{]} \backslash \backslash \mathrm{Q}$

\section{$\mathrm{CH}_{3} \mathrm{SC} \cdot\left(\mathrm{OCH}\left(\mathrm{CH}_{3}\right)_{2}\right) \mathrm{SCH}_{3}(14)$}

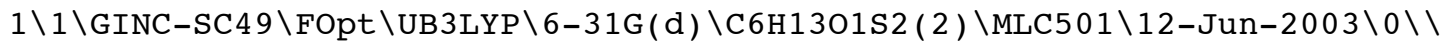
\# B3LYP/6-31G* OPT $=($ MAXCYC $=100)$ FREQ MAXDISK=134217728 GEOM=CHECK GUES $\mathrm{S}=\mathrm{READ} \backslash \backslash \mathrm{rrad} z=\mathrm{opr} r=\mathrm{me}-\mathrm{a} 1 \mathrm{~b} 1 \mathrm{c} 1 \mathrm{~d} 3 \mathrm{e} 1 \mathrm{f} 1-6 \mathrm{dub} \backslash \backslash \backslash 0,2 \backslash \mathrm{S}, 1.6328019823,1.38840$ $1723,-0.1049 \overline{8} 9024 \backslash \overline{\mathrm{C}}, 0.4962064126,0.0853734886,0.2517209429 \backslash \mathrm{S}, 1.1988888$ $212,-1.4468685145,0.7847054864 \backslash \mathrm{C}, 1.5098017786,-2.3335346769,-0.8095250$ $991 \backslash \mathrm{C}, 0.5054636028,2.8082485426,-0.3591364913 \backslash 0,-0.6550688454,0.101328$ $8175,-0.490018896 \backslash \mathrm{C},-1.8541633765,-0.4485441202,0.1239514117 \backslash \mathrm{H},-0.0546$ $290771,3.0257076897,0.5539093457 \backslash \mathrm{H}, 1.1402618391,3.662826288,-0.6069598$ $626 \backslash \mathrm{H},-0.1826352,2.6091653962,-1.1816575362 \backslash \mathrm{H}, 0.6082856175,-2.32249988$ $96,-1.4256007749 \backslash \mathrm{H}, 1.7644760353,-3.3656333436,-0.5536231302 \backslash \mathrm{H}, 2.338988$ $0911,-1.8731468445,-1.3515149837 \backslash \mathrm{C},-2.8466427355,-0.6420464264,-1.0142$ $666934 \backslash \mathrm{C},-2.3630065028,0.4846642067,1.2182888647 \backslash \mathrm{H},-1.5907540546,-1.41$ $91770644,0.5610261847 \backslash \mathrm{H},-3.7739747164,-1.0858305628,-0.6364213457 \backslash \mathrm{H},-3$ $.0893532393,0.3187454166,-1.4818233384 \backslash \mathrm{H},-2.4333910582,-1.3033608673,-$ $1.7822933569 \backslash \mathrm{H},-3.2544104671,0.0613944008,1.6949712795 \backslash \mathrm{H},-1.5986473683$ $, 0.6288005873,1.9885485942 \backslash \mathrm{H},-2.6266735704,1.4628808307,0.7999290815 \backslash \backslash$ Version=DEC-AXP-OSF $/ 1-G 03 R e v B .03 \backslash$ State $=2-A \backslash H F=-1108.0164465 \backslash \mathrm{S} 2=0.75462$ $8 \backslash S 2-1=0 . \backslash S 2 A=0.750016 \backslash R M S D=4.287 e-09 \backslash R M S F=3.572 e-06 \backslash D i p o l e=-0.8829033$ $, 0.0665324,-0.4079764 \backslash \mathrm{PG}=\mathrm{C} 01 \quad[\mathrm{X}(\mathrm{C} 6 \mathrm{H} 1301 \mathrm{~S} 2)] \backslash \backslash @$

\section{$\mathrm{CH}_{3} \mathrm{SCH}\left(\mathrm{OCH}\left(\mathrm{CH}_{3}\right)_{2}\right) \mathrm{SCH}_{3}$}

$1 \backslash 1 \backslash G I N C-S C 118 \backslash F O p t \backslash R B 3 L Y P \backslash 6-31 G(d) \backslash C 6 H 1401 S 2 \backslash M L C 501 \backslash 12-A u g-2003 \backslash 0 \backslash \backslash \#$ B3LYP $/ 6-31 \mathrm{G} *$ OPT $=($ MAXCYC $=100, \mathrm{TIGHT})$ FREQ MAXDISK $=52428800 \backslash \backslash \mathrm{ralk} z=0 \mathrm{pr}$ $r=m e-a 1 b 1 c 1 d 3 e 1 f 1-6 d u b 3 \backslash \backslash 0,1 \backslash S, 1.6930291365,1.2589363122,-0.142891974 \overline{2}$ $\backslash \mathrm{C}, 0.4375226526,0.0793900766,0.4934764623 \backslash \mathrm{S}, 1.20046656,-1.5997048759,0$ $.663686937 \backslash \mathrm{C}, 1.491995356,-2.0094837548,-1.0969870558 \backslash \mathrm{C}, 0.6743053347,2$. $7719138932,-0.2872626609 \backslash 0,-0.684311094,0.1089078389,-0.3417065141 \backslash \mathrm{C},-$ $1.918778926,-0.3568115456,0.2565043605 \backslash \mathrm{H}, 0.330143932,3.1186583272,0.69$ $19583372 \backslash \mathrm{H}, 1.3194018473,3.5384513149,-0.7252305649 \backslash \mathrm{H},-0.1790782806,2.5$ $94095418,-0.9433533526 \backslash \mathrm{H}, 0.6150331273,-1.7324315382,-1.6857794253 \backslash \mathrm{H}, 1$. $6384615219,-3.0913262949,-1.1471048578 \backslash \mathrm{H}, 2.3763191191,-1.5016954769,-1$ $.4862850442 \backslash \mathrm{C},-2.7802651381,-0.8714758186,-0.8902464403 \backslash \mathrm{C},-2.591732288$ $6,0.7726842136,1.0367932541 \backslash \mathrm{H},-1.6800217388,-1.1896794524,0.9314564832$ $\backslash \mathrm{H},-3.743321217,-1.2333853501,-0.5138885176 \backslash \mathrm{H},-2.9711228079,-0.0710662$ $142,-1.613944164 \backslash \mathrm{H},-2.2795768321,-1.6936831193,-1.410335575 \backslash \mathrm{H},-3.51906$ $81492,0.4169330554,1.5000361824 \backslash \mathrm{H},-1.9473458712,1.1530733312,1.8369938$ $535 \backslash \mathrm{H},-2.8367898646,1.6052625578,0.368064295 \backslash \mathrm{H}, 0.1772408774,0.35052536$ $36,1.5246775387 \backslash \backslash$ Version=DEC-AXP-OSF / 1-G03RevB.03 \State $=1-\mathrm{A} \backslash \mathrm{HF}=-1108.6$ $634841 \backslash \mathrm{RMSD}=5.970 \mathrm{e}-09 \backslash \mathrm{RMSF}=3.186 \mathrm{e}-06 \backslash \mathrm{Dipole}=-0.9898547,0.2398973,-0.10$ $49077 \backslash \mathrm{PG}=\mathrm{C} 01 \quad[\mathrm{X}(\mathrm{C} 6 \mathrm{H} 1401 \mathrm{~S} 2)] \backslash \backslash \mathrm{Q}$

\section{$\mathrm{S}=\mathrm{C}\left(\mathrm{OCH}\left(\mathrm{CH}_{3}\right)_{2}\right) \mathrm{SCH}_{3}$}

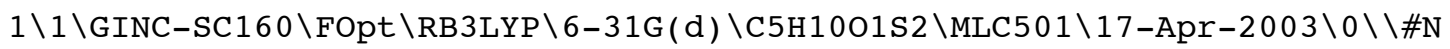

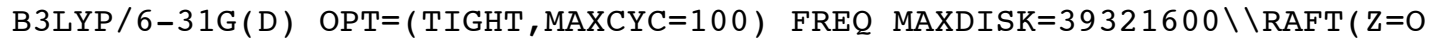
pr $\mathrm{R}=\mathrm{Me}) \mathrm{a} 1 \mathrm{~b} 1 \backslash \backslash 0,1 \backslash 0,-0.1880409305,-0.1960063398,-0.9146059654 \backslash \mathrm{C},-0.16$ $34698107,0.1208318952,0.3796378951 \backslash \mathrm{S}, 1.0989419575,0.7518398963,1.24169$ 
$43287 \backslash \mathrm{S},-1.7804771858,-0.2656031225,1.0071850584 \backslash \mathrm{C}, 0.9905955826,-0.022$ $3839841,-1.7694161684 \backslash \mathrm{C},-1.6426563686,0.2008613435,2.760975016 \backslash \mathrm{H},-0.86$ $51536385,-0.3828064605,3.256969389 \backslash \mathrm{H},-1.4238995035,1.2651146149,2.8631$ $670358 \backslash \mathrm{H},-2.6185788355,-0.0249825208,3.2001517599 \backslash \mathrm{C}, 0.4424182525,0.298$ $0059902,-3.1523519357 \backslash \mathrm{C}, 1.8249584935,-1.2958520616,-1.716083723 \backslash \mathrm{H}, 1.55$ $88488582,0.8208918648,-1.3688382623 \backslash \mathrm{H}, 1.2719818992,0.456344208,-3.8498$ $534733 \backslash \mathrm{H},-0.173003433,-0.5258789427,-3.5292417693 \backslash \mathrm{H},-0.1682295917,1.20$ $5720672,-3.129689072 \backslash \mathrm{H}, 2.7021884098,-1.1904102236,-2.3641930663 \backslash \mathrm{H}, 2.17$ $3627284,-1.489670749,-0.6980809696 \backslash \mathrm{H}, 1.2400327531,-2.154839226,-2.0621$ $80545 \backslash \backslash$ Version=DEC-AXP-OSF / 1-G98RevA.11.3\HF=-1068.1612165 \RMSD=4.100e $-09 \backslash \mathrm{RMSF}=2.255 \mathrm{e}-06 \backslash \mathrm{Dipole}=-0.1299334,-0.1819352,-0.4116629 \backslash \mathrm{PG}=\mathrm{C} 01] \mathrm{X}(\mathrm{C}$ $5 \mathrm{H} 1001 \mathrm{~S} 2$ ) ] \\@

\section{$\mathrm{CH}_{3} \mathrm{SC} \cdot\left(\mathrm{OC}\left(\mathrm{CH}_{3}\right)_{3}\right) \mathrm{SCH}_{3}(15)$}

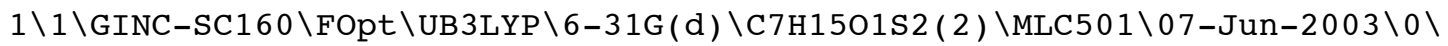
\# B3LYP/6-31G* OPT= (MAXCYC $=100$, TIGHT $)$ FREQ MAXDISK=134217728 \\rrad_z=

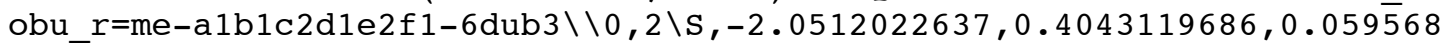
$458 \backslash \mathrm{C},-0.2713212906,0.3951909451,0.0514592196 \backslash \mathrm{S}, 0.6989830765,1.8799943$ $449,0.0390201419 \backslash \mathrm{C}, 1.0396518435,2.0953201548,-1.762913683 \backslash \mathrm{C},-2.4463174$ $333,1.8516800748,1.0991214788 \backslash 0,0.2065666959,-0.6894446763,-0.62190883$ $97 \backslash \mathrm{C}, 0.8563163854,-1.7948532634,0.1134355883 \backslash \mathrm{H},-2.0689489292,1.7179491$ $3,2.1161526517 \backslash \mathrm{H},-2.0302146546,2.7648940145,0.6689788998 \backslash \mathrm{H},-3.53725161$ $91,1.9189968361,1.123693429 \backslash \mathrm{H}, 1.4267109218,1.1607332199,-2.1728311658 \backslash$ $\mathrm{H}, 1.7958175349,2.8799245947,-1.8517850001 \backslash \mathrm{H}, 0.1336304584,2.3929850132$, $-2.2964197635 \backslash \mathrm{C}, 1.151801511,-2.8085123953,-0.9922402886 \backslash \mathrm{C},-0.109591107$ $9,-2.3719666749,1.1504934401 \backslash \mathrm{C}, 2.1474749123,-1.2979283953,0.7692354866$ $\backslash \mathrm{H}, 1.6424448654,-3.6945195462,-0.5753226558 \backslash \mathrm{H}, 0.2244730777,-3.12141730$ $42,-1.4823999081 \backslash \mathrm{H}, 1.8109579389,-2.3705732993,-1.7488246031 \backslash \mathrm{H}, 0.354486$ $7996,-3.2257665395,1.6571836934 \backslash \mathrm{H},-0.3666732949,-1.6247122904,1.907308$ $7858 \backslash \mathrm{H},-1.035104744,-2.7120871675,0.6756677705 \backslash \mathrm{H}, 2.6712805681,-2.13659$ $77065,1.242495543 \backslash \mathrm{H}, 2.8134906867,-0.8495325418,0.0254194289 \backslash \mathrm{H}, 1.939784$ $8958,-0.5472026932,1.5369885625 \backslash \backslash$ Version=DEC-AXP-OSF / 1-G03RevB.03\Stat $\mathrm{e}=2-\mathrm{A} \backslash \mathrm{HF}=-1147.3279774 \backslash \mathrm{S} 2=0.754752 \backslash \mathrm{S} 2-1=0 . \backslash \mathrm{S} 2 \mathrm{~A}=0.750017 \backslash \mathrm{RMSD}=9.563 e-09$ $\backslash \mathrm{RMSF}=1.806 \mathrm{e}-06 \backslash \mathrm{Dipole}=0.1685338,-0.0656418,0.098614 \backslash \mathrm{PG}=\mathrm{C} 01 \quad[\mathrm{X}(\mathrm{C} 7 \mathrm{H} 1501$ $\mathrm{S} 2)] \backslash \backslash @$

\section{$\mathrm{CH}_{3} \mathrm{SCH}\left(\mathrm{OC}\left(\mathrm{CH}_{3}\right)_{3}\right) \mathrm{SCH}_{3}$}

$1 \backslash 1 \backslash$ GINC-SC15 $\backslash$ FOpt $\backslash R B 3 L Y P \backslash 6-31 G(d) \backslash C 7 H 1601 S 2 \backslash M L C 501 \backslash 13-A u g-2003 \backslash 0 \backslash \backslash \#$ B 3LYP $/ 6-31 \mathrm{G} *$ OPT $=($ MAXCYC $=100$, TIGHT $)$ FREQ GEOM=CHECK GUESS=READ MAXDISK=

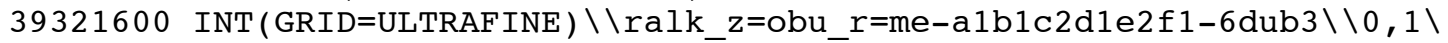
$\mathrm{S},-1.43457935,-1.3310505082,-0.656 \overline{8} 23871 \backslash \mathrm{C},-0.4153438291,-0.098620019$, $0.2833477177 \backslash \mathrm{S},-1.2795458005,1.481939106,0.7031927047 \backslash \mathrm{C},-1.5062405121$, $2.1692335989,-0.9741447117 \backslash \mathrm{C},-2.812611971,-1.6024204183,0.510279272 \backslash 0$, $0.7085112104,0.1184559369,-0.5144179196 \backslash \mathrm{C}, 2.0344275266,-0.1606648331,0$ $.029778393 \backslash \mathrm{H},-2.4642685217,-2.0439932872,1.4496163615 \backslash \mathrm{H},-3.3394322226$, $-0.667405649,0.7161031559 \backslash \mathrm{H},-3.4963060743,-2.3045998223,0.0252696801 \backslash \mathrm{H}$ $,-0.5376150118,2.2895082598,-1.4630618223 \backslash \mathrm{H},-1.9820941703,3.1453949104$ $,-0.8524566556 \backslash \mathrm{H},-2.1478358406,1.5197310942,-1.5744858734 \backslash \mathrm{C}, 2.96200970$ $34,0.2110855292,-1.1298784575 \backslash \mathrm{C}, 2.1698457848,-1.6513365806,0.368142572$ $7 \backslash \mathrm{C}, 2.3178319,0.7252597439,1.2504501235 \backslash \mathrm{H}, 4.0080521608,0.039318431,-0$. $8548082024 \backslash \mathrm{H}, 2.7286234775,-0.3927509035,-2.012363615 \backslash \mathrm{H}, 2.8396676299,1$. $2666536996,-1.392728589 \backslash \mathrm{H}, 3.1946178961,-1.871639565,0.6874491777 \backslash \mathrm{H}, 1.5$ $015052877,-1.9514065098,1.1825374653 \backslash \mathrm{H}, 1.9366539524,-2.2655874818,-0.5$ $066927333 \backslash \mathrm{H}, 3.350027804,0.5770169987,1.587366913 \backslash \mathrm{H}, 2.1800862086,1.7810$ $85605,0.9993968161 \backslash \mathrm{H}, 1.6595424306,0.4888022999,2.0931076706 \backslash \mathrm{H},-0.17282$ $38969,-0.5272152666,1.2613428094 \backslash \backslash$ Version=DEC-AXP-OSF / 1-G03RevB.03 \Sta $t e=1-A \backslash H F=-1147.9760733 \backslash R M S D=3.758 e-09 \backslash R M S F=8.446 e-07 \backslash D i p o l e=0.3531735$ 
$,-0.1072806,0.3244109 \backslash \mathrm{PG}=\mathrm{C} 01 \quad[\mathrm{X}(\mathrm{C} 7 \mathrm{H} 1601 \mathrm{~S} 2)] \backslash \backslash @$

\section{$\mathrm{S}=\mathrm{C}\left(\mathrm{OC}\left(\mathrm{CH}_{3}\right)_{3}\right) \mathrm{SCH}_{3}$}

$1 \backslash 1 \backslash G I N C-S C 11 \backslash F O p t \backslash R B 3 L Y P \backslash 6-31 G(d) \backslash C 6 H 1201 S 2 \backslash M L C 501 \backslash 12-M a r-2003 \backslash 0 \backslash \backslash \# N$ $\mathrm{B} 3 \mathrm{LYP} / 6-31 \mathrm{G}$ (D) OPT= $(\mathrm{TIGHT}, \mathrm{MAXCYC}=100)$ FREQ MAXDISK=39321600 $\backslash \mathrm{RAFT}(\mathrm{Z}=\mathrm{OB}$ $\mathrm{u} R=\mathrm{CH} 3$ ) lowest $\mathrm{a} 1 \mathrm{~b} 1$ in $\mathrm{Cs}$ symm $\backslash \backslash 0,1 \backslash \mathrm{C},-2.8094762742,-1.973078311,0 . \backslash \mathrm{S}$ $,-0.9909272249,-2.0266499924,0 . \backslash \mathrm{C},-0.5463827548,-0.2981117822,0 . \backslash \mathrm{S},-1$. $6190708437,0.9612038617,0 . \backslash 0,0.7824311486,-0.289547251,0 . \backslash \mathrm{C}, 1.68430479$ $23,0.8956108337,0 . \backslash \mathrm{H},-3.1313924883,-3.0185738969,0 . \backslash \mathrm{H},-3.1833163326,-1$ $.4669879944,0.8918110025 \backslash \mathrm{H},-3.1833163326,-1.4669879944,-0.8918110025 \backslash \mathrm{C}$ $, 1.4810774164,1.711623448,1.2779179042 \backslash \mathrm{C}, 1.4810774164,1.711623448,-1.2$ $779179042 \backslash \mathrm{H}, 0.506186353,2.2002055556,1.2986143138 \backslash \mathrm{H}, 0.506186353,2.2002$ $055556,-1.2986143138 \backslash \mathrm{H}, 1.5747551593,1.0694331401,2.1599032388 \backslash \mathrm{H}, 1.5747$ $551593,1.0694331401,-2.1599032388 \backslash \mathrm{H}, 2.2586679702,2.4818914203,1.333659$ $7933 \backslash \mathrm{H}, 2.2586679702,2.4818914203,-1.3336597933 \backslash \mathrm{C}, 3.0603127374,0.224269$ $7459,0 . \backslash \mathrm{H}, 3.8449479523,0.9877323386,0 . \backslash \mathrm{H}, 3.1844490722,-0.4031754401,0$. $8879541515 \backslash \mathrm{H}, 3.1844490722,-0.4031754401,-0.8879541515 \backslash \backslash$ Version=DEC-AXP -OSF / 1-G98RevA.11.3\State=1-A ' $\backslash \mathrm{HF}=-1107.4708739 \backslash \mathrm{RMSD}=9.707 \mathrm{e}-09 \backslash \mathrm{RMSF}=1$. $552 \mathrm{e}-06 \backslash \mathrm{Dipole}=0.5651463,-0.0509505,0 . \backslash \mathrm{PG}=\mathrm{CS} \quad[\mathrm{SG}(\mathrm{C} 4 \mathrm{H} 2 \mathrm{O} 1 \mathrm{~S} 2), \mathrm{X}(\mathrm{C} 2 \mathrm{H} 10)] \backslash \backslash$ (a 
Table S2. Geometries of the RAFT-adduct Radicals, and Corresponding Alkanes

and Thiocarbonyl Products of Beta-Scission, Used in Calculating the Radical

Stabilization Energies and Beta-Scission Enthalpies in Table 2

\section{$\mathrm{CH}_{3} \mathrm{SC} \cdot\left(\mathrm{CH}_{3}\right) \mathrm{S}-\mathrm{CH}_{2} \mathrm{CN}(16)$}

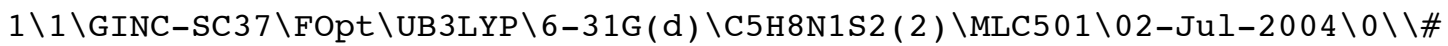

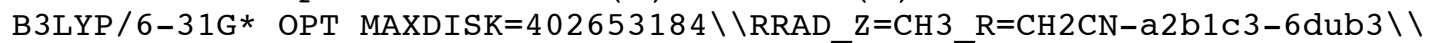
$0,2 \backslash \mathrm{S},-1.5380304454,-0.2524762319,-1.1514997921 \bar{C}_{,}, 0.7404267942,-0.869$ $7341876,0.2805413448 \backslash \mathrm{S}, 0.988148005,-0.9842143591,0.4748666674 \backslash \mathrm{C},-1.661$ $823209,-1.2034376129,1.423317539 \backslash \mathrm{C},-0.2024466317,0.1912427838,-2.31077$ $13878 \backslash \mathrm{C}, 1.4619280315,0.5573715331,1.4620467877 \backslash \mathrm{H}, 2.484508004,0.3682299$ $976,1.8036453886 \backslash \mathrm{H}, 0.8142951832,0.6389240576,2.3388328927 \backslash \mathrm{C}, 1.41091556$ $14,1.776935843,0.6703974501 \backslash \mathrm{H},-1.3441427705,-2.128799659,1.9167691322 \backslash$ $\mathrm{H},-2.6950123323,-1.3342674304,1.0814345642 \backslash \mathrm{H},-1.6728418189,-0.40980979$ $79,2.1870131768 \backslash \mathrm{H},-0.7091891149,0.5167715148,-3.2230978858 \backslash \mathrm{H}, 0.4008835$ $366,1.010448331,-1.9152679157 \backslash \mathrm{H}, 0.4266889358,-0.6732297701,-2.53098970$ $22 \backslash \mathrm{N}, 1.3548639536,2.726358865,0.0020857058 \backslash \backslash$ Version=DEC-AXP-OSF / 1-G03R evB.03 $\backslash$ state $=2-A \backslash H F=-1046.412022 \backslash S 2=0.755354 \backslash S 2-1=0 . \backslash S 2 A=0.750022 \backslash R M S D$ $=4.672 \mathrm{e}-09 \backslash \mathrm{RMSF}=7.274 \mathrm{e}-06 \backslash \mathrm{Dipole}=-0.0365961,-0.7579938,0.5856023 \backslash \mathrm{PG}=\mathrm{C} 0$ $1[\mathrm{X}(\mathrm{C} 5 \mathrm{H} 8 \mathrm{~N} 1 \mathrm{~S} 2)] \backslash \backslash @$

\section{$\mathrm{CH}_{3} \mathrm{SCH}\left(\mathrm{CH}_{3}\right) \mathrm{S}-\mathrm{CH}_{2} \mathrm{CN}$}

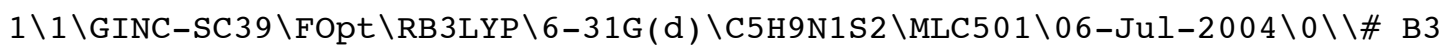
$\mathrm{LYP} / 6-31 \mathrm{G} * \mathrm{OPT}=(\mathrm{TIGHT}, \mathrm{MAXCYC}=200)$ FREQ SCF=TIGHT MAXDISK $=402653184$ GEO $\mathrm{M}=\mathrm{CHECK}$ GUESS=READ $\backslash \backslash \mathrm{RALK} Z \mathrm{Z}=\mathrm{CH} 3 \_\mathrm{R}=\mathrm{CH} 2 \mathrm{CN}-\mathrm{a} 2 \mathrm{~b} 1 \mathrm{c} 3-6 \mathrm{dub} 3 \backslash \backslash 0,1 \backslash \mathrm{S}, 1.030488145$ $1,1.2341481866,-0.5620926539 \backslash \mathrm{C}, 1.3325757555,-0.375152743,0.2631794691 \backslash$ $\mathrm{S},-0.1660239863,-1.2241836594,0.9643213227 \backslash \mathrm{C}, 2.1200318679,-1.270384045$ $8,-0.6974429416 \backslash \mathrm{C}, 0.2975471295,2.2179707557,0.7900289247 \backslash \mathrm{C},-1.32671688$ $98,-1.2252133382,-0.4713585602 \backslash \mathrm{H},-1.8674828742,-2.1758149145,-0.438132$ $2286 \backslash \mathrm{H},-0.7582388037,-1.1927694808,-1.405092527 \backslash \mathrm{C},-2.2850444582,-0.121$ $4537478,-0.4393859344 \backslash \mathrm{H}, 2.3638912523,-2.2195930559,-0.2123693078 \backslash \mathrm{H}, 3.0$ $510772327,-0.7771113318,-0.9973344153 \backslash \mathrm{H}, 1.5493725849,-1.4875789786,-1$. $6069647012 \backslash \mathrm{H}, 0.1487041814,3.2232500216,0.3875520418 \backslash \mathrm{H},-0.6680028684,1$. $8097209479,1.0957437862 \backslash \mathrm{H}, 0.9753216952,2.2744372587,1.646689472 \backslash \mathrm{N},-3.0$ $544433973,0.7470449941,-0.3919731554 \backslash \mathrm{H}, 1.9246744121,-0.1978891462,1.16$ $79355208 \backslash \backslash$ Version=DEC-AXP-OSF $/ 1-G 03$ RevB.03 S State $=1-A \backslash H F=-1047.0586754 \backslash$ $\mathrm{RMSD}=5.358 \mathrm{e}-09 \backslash \mathrm{RMSF}=2.316 \mathrm{e}-06 \backslash \mathrm{Dipole}=1.1198016,-0.8095285,0.0379239 \backslash \mathrm{PG}$ $=\mathrm{C} 01[\mathrm{X}(\mathrm{C} 5 \mathrm{H} 9 \mathrm{~N} 1 \mathrm{~S} 2)] \backslash \backslash @$

\section{$\mathrm{S}=\mathrm{C}\left(\mathrm{CH}_{3}\right) \mathrm{S}-\mathrm{CH}_{2} \mathrm{CN}$}

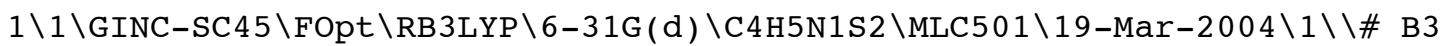
LYP $/ 6-31 \mathrm{G} * \mathrm{FOPT}=(\mathrm{MAXCYC}=300, \mathrm{Z}-\mathrm{MATRIX})$ MAXDISK $=134217728 \backslash \backslash \mathrm{RAFT} Z=\mathrm{ZH} 3 \_\mathrm{R}=$ $\mathrm{CH} 2 \mathrm{CN}-\mathrm{a} 3 \mathrm{~b} 1-6 \mathrm{dub} 3 \backslash \backslash 0,1 \backslash \mathrm{C} \backslash \mathrm{C}, 1, \mathrm{~B} 1 \backslash \mathrm{S}, 2, \mathrm{~B} 2,1, \mathrm{~A} 1 \backslash \mathrm{S}, 2, \mathrm{~B} 3,1, \mathrm{~A} 2,3, \mathrm{D} 1,0 \backslash \mathrm{C}, 4, \mathrm{~B} 4,2$ , A3 , $1, \mathrm{D} 2,0 \backslash \mathrm{C}, 5, \mathrm{~B} 5,4, \mathrm{~A} 4,2, \mathrm{D} 3,0 \backslash \mathrm{H}, 5, \mathrm{~B} 6,4, \mathrm{~A} 5,6, \mathrm{D} 4,0 \backslash \mathrm{H}, 5, \mathrm{~B} 7,4, \mathrm{~A} 6,6, \mathrm{D} 5,0 \backslash \mathrm{H}$, 1 , $\mathrm{B} 8,2, \mathrm{~A} 7,3, \mathrm{D} 6,0 \backslash \mathrm{H}, 1, \mathrm{~B} 9,2, \mathrm{~A} 8,9, \mathrm{D} 7,0 \backslash \mathrm{H}, 1, \mathrm{~B} 10,2, \mathrm{~A} 9,9, \mathrm{D} 8,0 \backslash \mathrm{X}, 6,1 ., 5,90.4$ , D9 , $0 \backslash N, 6, B 11,12, \mathrm{~A} 10,5,180.0 \backslash \backslash \mathrm{B} 1=1.51416247 \backslash \mathrm{B} 2=1.64284125 \backslash \mathrm{B} 3=1.761076$ $58 \backslash B 4=1.8403095 \backslash B 5=1.45879272 \backslash B 6=1.09433674 \backslash B 7=1.09441266 \backslash B 8=1.0954722$ $4 \backslash B 9=1.09682466 \backslash B 10=1.09245527 \backslash B 11=1.16042021 \backslash A 1=123.37991907 \backslash A 2=110.7$ $4648554 \backslash A 3=102.0502169 \backslash A 4=109.36720461 \backslash A 5=109.0929812 \backslash A 6=108.97689249 \backslash$ $\mathrm{A} 7=112.7778099 \backslash \mathrm{A} 8=108.89778025 \backslash \mathrm{A} 9=109.71152501 \backslash \mathrm{A} 10=89.81567531 \backslash \mathrm{D} 1=-178$ $.57873247 \backslash \mathrm{D} 2=177.17630707 \backslash \mathrm{D} 3=-180.48291408 \backslash \mathrm{D} 4=121.33196566 \backslash \mathrm{D} 5=-121.292$ $88244 \backslash D 6=-160.12420209 \backslash D 7=-119.9522014 \backslash D 8=122.30311959 \backslash D 9=-171.3058611$ $\backslash \backslash$ Version=DEC-AXP-OSF $/ 1-G 03 R e v B .03 \backslash$ State $=1-A \backslash H F=-1006.5367763 \backslash \mathrm{RMSD}=2.5$ $18 e-09 \backslash \mathrm{RMSF}=2.636 \mathrm{e}-05 \backslash \mathrm{Dipole}=0.6227997,-0.0165881,-1.0213479 \backslash \mathrm{PG}=\mathrm{C} 01] \mathrm{X}$ 


\section{(C4H5N1S2) ] \\@}

\section{$\mathrm{CH}_{3} \mathrm{SC} \cdot\left(\mathrm{CH}_{3}\right) \mathrm{S}-\mathrm{C}\left(\mathrm{CH}_{3}\right)_{2} \mathrm{CN}(17)$}

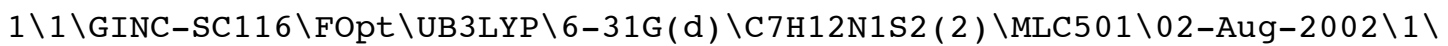
$\backslash \# \mathrm{~N}$ UB3LYP $/ 6-31 \mathrm{G}(\mathrm{D}) \mathrm{OPT}=(\mathrm{Z}-\mathrm{MATRIX}, \mathrm{MAXCYC}=100) \mathrm{SCF}=(\mathrm{MAXCYC}=100, \mathrm{QC}, \mathrm{TIGHT}$ ) FREQ MAXDISK=131072000\\RAFT_RAD( $Z=M e ~ R=M e 2 C C N) c 1 b / / B 3 \backslash \backslash 0,2 \backslash C \backslash C, 1, B$ $1 \backslash \mathrm{S}, 1, \mathrm{~B} 2,2, \mathrm{~A} 1 \backslash \mathrm{S}, 1, \mathrm{~B} 3,2, \mathrm{~A} 2,3, \mathrm{D} 1,0 \backslash \mathrm{C}, 3, \mathrm{~B} 4,1, \mathrm{~A} 3,2, \mathrm{D} 2,0 \backslash \mathrm{C}, 4, \mathrm{~B} 5,1, \mathrm{~A} 4,2, \mathrm{D} 3,0$ $\backslash \mathrm{C}, 6, \mathrm{~B} 6,4, \mathrm{~A} 5,1, \mathrm{D} 4,0 \backslash \mathrm{C}, 6, \mathrm{~B} 7,4, \mathrm{~A} 6,1, \mathrm{D} 5,0 \backslash \mathrm{C}, 6, \mathrm{~B} 8,4, \mathrm{~A} 7,1, \mathrm{D} 6,0 \backslash \mathrm{H}, 5, \mathrm{~B} 9,3, \mathrm{~A} 8$, $1, \mathrm{D} 7,0 \backslash \mathrm{H}, 5, \mathrm{~B} 10,3, \mathrm{~A} 9,10, \mathrm{D} 8,0 \backslash \mathrm{H}, 5, \mathrm{~B} 11,3, \mathrm{~A} 10,10, \mathrm{D} 9,0 \backslash \mathrm{H}, 2, \mathrm{~B} 12,1, \mathrm{~A} 11,3, \mathrm{D} 10$, $0 \backslash \mathrm{H}, 2, \mathrm{~B} 13,1, \mathrm{~A} 12,13, \mathrm{D} 11,0 \backslash \mathrm{H}, 2, \mathrm{~B} 14,1, \mathrm{~A} 13,13, \mathrm{D} 12,0 \backslash \mathrm{H}, 7, \mathrm{~B} 15,6, \mathrm{~A} 14,4, \mathrm{D} 13,0 \backslash$ $\mathrm{H}, 7, \mathrm{~B} 16,6, \mathrm{~A} 15,16, \mathrm{D} 14,0 \backslash \mathrm{H}, 7, \mathrm{~B} 17,6, \mathrm{~A} 16,16, \mathrm{D} 15,0 \backslash \mathrm{H}, 8, \mathrm{~B} 18,6, \mathrm{~A} 17,4, \mathrm{D} 16,0 \backslash \mathrm{H}$, $8, \mathrm{~B} 19,6, \mathrm{~A} 18,19, \mathrm{D} 17,0 \backslash \mathrm{H}, 8, \mathrm{~B} 20,6, \mathrm{~A} 19,19, \mathrm{D} 18,0 \backslash \mathrm{X}, 9,1 ., 6,90.4, \mathrm{D} 19,0 \backslash \mathrm{N}, 9$, $\mathrm{B}$ $21,22, \mathrm{~A} 20,6,180,0 \backslash \backslash \mathrm{D} 2=16.38295078 \backslash \mathrm{D} 3=77.64088756 \backslash \mathrm{D} 4=60.37483458 \backslash \mathrm{D} 5=-1$ $78.7383868 \backslash \mathrm{D} 6=-61.16988989 \backslash \mathrm{B} 1=1.50208945 \backslash \mathrm{B} 2=1.75657198 \backslash \mathrm{B} 3=1.74732399 \backslash \mathrm{B}$ $4=1.83034897 \backslash \mathrm{B} 5=1.91282884 \backslash \mathrm{B} 6=1.53539307 \backslash \mathrm{B} 7=1.5395273 \backslash \mathrm{B} 8=1.46607825 \backslash \mathrm{B} 9$ $=1.09303629 \backslash \mathrm{B} 10=1.092321 \backslash \mathrm{B} 11=1.09293827 \backslash \mathrm{B} 12=1.10007771 \backslash \mathrm{B} 13=1.09890754 \backslash$ $\mathrm{B} 14=1.09212294 \backslash \mathrm{B} 15=1.09292583 \backslash \mathrm{B} 16=1.09396533 \backslash \mathrm{B} 17=1.09672447 \backslash \mathrm{B} 18=1.0945$ $1538 \backslash \mathrm{B} 19=1.09522691 \backslash \mathrm{B} 20=1.09354597 \backslash \mathrm{B} 21=1.16247912 \backslash \mathrm{A} 1=122.50686981 \backslash \mathrm{A} 2=1$ $23.17398239 \backslash A 3=104.46655439 \backslash A 4=106.49858295 \backslash A 5=110.98235599 \backslash A 6=104.639$ $96652 \backslash A 7=108.61843008 \backslash A 8=105.70641764 \backslash A 9=111.77488663 \backslash A 10=111.17328243$ $\backslash A 11=112.07996327 \backslash A 12=111.90892444 \backslash A 13=110.10196343 \backslash A 14=111.36166171 \backslash A$ $15=109.52774174 \backslash A 16=110.12678 \backslash A 17=109.62280482 \backslash A 18=109.87509857 \backslash A 19=11$ $1.407113 \backslash \mathrm{A} 20=89.82090986 \backslash \mathrm{D} 1=-177.90491864 \backslash \mathrm{D} 7=180.96073931 \backslash \mathrm{D} 8=118.42528$ $654 \backslash D 9=-118.08928553 \backslash D 10=64.15148444 \backslash D 11=-120.40351119 \backslash D 12=119.4744608$ $\backslash \mathrm{D} 13=-60.04758136 \backslash \mathrm{D} 14=120.04550823 \backslash \mathrm{D} 15=-120.27316252 \backslash \mathrm{D} 16=-61.18845374 \backslash$ $\mathrm{D} 17=-119.2157694 \backslash \mathrm{D} 18=120.55449916 \backslash \mathrm{D} 19=166.9636757 \backslash \backslash$ Version=DEC $-\mathrm{AXP}-\mathrm{OSF}$ /1-G98RevA. $11.3 \backslash \mathrm{HF}=-1125.0409306 \backslash \mathrm{S} 2=0.755753 \backslash \mathrm{S} 2-1=0 . \backslash \mathrm{S} 2 \mathrm{~A}=0.750025 \backslash \mathrm{RMSD}$ $=0.000 e+00 \backslash \mathrm{RMSF}=9.570 \mathrm{e}-05 \backslash \mathrm{Dipole}=0.9525901,-0.1520827,-0.5410097 \backslash \mathrm{PG}=\mathrm{C} 0$ $1[\mathrm{X}(\mathrm{C} 7 \mathrm{H} 12 \mathrm{~N} 1 \mathrm{~S} 2)] \backslash \backslash \mathrm{Q}$

\section{$\mathrm{CH}_{3} \mathrm{SCH}\left(\mathrm{CH}_{3}\right) \mathrm{S}-\mathrm{C}\left(\mathrm{CH}_{3}\right)_{2} \mathrm{CN}$}

$1 \backslash 1 \backslash G I N C-S C 14 \backslash F O p t \backslash R B 3 L Y P \backslash 6-31 G(d) \backslash C 7 H 13 N 1 S 2 \backslash M L C 501 \backslash 16-J a n-2003 \backslash 0 \backslash \backslash \# N$

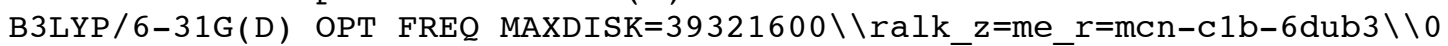
$, 1 \backslash \mathrm{C}, 0.4023252859,0.3483128389,-1.2517259945 \backslash \mathrm{C}, 1.83 \overline{3} 1598532,0.40213615$ $61,-0.7196520928 \backslash \mathrm{S},-0.5298787499,1.944874156,-1.0866003884 \backslash \mathrm{S},-0.629638$ $0182,-1.0532435388,-0.6390291613 \backslash \mathrm{C}, 0.4760088835,3.037750853,-2.1553107$ $136 \backslash \mathrm{C},-0.4957641124,-1.1408537855,1.2268313845 \backslash \mathrm{C},-0.8758522961,0.17193$ $09586,1.9353520648 \backslash \mathrm{C},-1.486506521,-2.2663172647,1.6082541483 \backslash \mathrm{C}, 0.85798$ $7673,-1.5604937808,1.6237406123 \backslash \mathrm{H},-0.0747030455,3.979164735,-2.2333009$ $886 \backslash \mathrm{H}, 1.4615112031,3.2438460019,-1.7295190403 \backslash \mathrm{H}, 0.5853837634,2.6158802$ $071,-3.1597478298 \backslash \mathrm{H}, 1.8670688314,0.665762979,0.3399445233 \backslash \mathrm{H}, 2.40715906$ $47,1.1547970819,-1.2710034215 \backslash \mathrm{H}, 2.3285974646,-0.5651288921,-0.84856515$ $79 \backslash \mathrm{H},-0.2007788889,0.9895091267,1.6759570542 \backslash \mathrm{H},-1.8902782671,0.4603286$ $472,1.6463546933 \backslash \mathrm{H},-0.8440497163,0.0244655886,3.0213685198 \backslash \mathrm{H},-2.502215$ $1784,-1.9716051394,1.3287495304 \backslash \mathrm{H},-1.4517945589,-2.4310771749,2.690095$ $6091 \backslash \mathrm{H},-1.2377754572,-3.2060123773,1.107358181 \backslash \mathrm{N}, 1.9154804945,-1.89934$ $91081,1.9657922311 \backslash \mathrm{H}, 0.427627016,0.1146272447,-2.3231009488 \backslash \backslash$ Version=D EC-AXP-OSF / 1-G98RevA. 11.3 \HF=-1125.682352 \RMSD=2 . 264e-09 \RMSF=1.143e-0 $5 \backslash \mathrm{Dipole}=-0.4777204,0.7267385,-0.457958 \backslash \mathrm{PG}=\mathrm{C} 01 \quad[\mathrm{X}(\mathrm{C} 7 \mathrm{H} 13 \mathrm{~N} 1 \mathrm{~S} 2)] \backslash \backslash \mathrm{a}$

\section{$\mathrm{S}=\mathrm{C}\left(\mathrm{CH}_{3}\right) \mathrm{S}-\mathrm{C}\left(\mathrm{CH}_{3}\right)_{2} \mathrm{CN}$}

$1 \backslash 1 \backslash G I N C-S C 70 \backslash F O p t \backslash R B 3 L Y P \backslash 6-31 G(d) \backslash C 6 H 9 N 1 S 2 \backslash M L C 501 \backslash 01-A u g-2002 \backslash 1 \backslash \backslash \# N B$ $3 \mathrm{LYP} / 6-31 \mathrm{G} * \mathrm{OPT}=(\mathrm{Z}-\mathrm{MATRIX}, \mathrm{MAXCYC}=100) \mathrm{SCF}=(\mathrm{TIGHT}, \mathrm{MAXCYC}=100)$ FREQ MAXD ISK $=131072000 \backslash \backslash \mathrm{RAFT}(\mathrm{Z}=\mathrm{Me} \mathrm{R}=\mathrm{Me} 2 \mathrm{CCN}) \mathrm{C} 1 \mathrm{x} / / \mathrm{B} 3 \backslash \backslash 0,1 \backslash \mathrm{S} \backslash \mathrm{C}, 1, \mathrm{~B} 1 \backslash \mathrm{S}, 2, \mathrm{~B} 2,1, \mathrm{~A} 1 \backslash \mathrm{C}$ $, 2, \mathrm{~B} 3,1, \mathrm{~A} 2,3, \mathrm{D} 1,0 \backslash \mathrm{H}, 4, \mathrm{~B} 4,2, \mathrm{~A} 3,1, \mathrm{D} 2,0 \backslash \mathrm{H}, 4, \mathrm{~B} 5,2, \mathrm{~A} 4,5, \mathrm{D} 3,0 \backslash \mathrm{H}, 4, \mathrm{~B} 6,2, \mathrm{~A} 5,5$, $\mathrm{D} 4,0 \backslash \mathrm{C}, 3, \mathrm{~B} 7,2, \mathrm{~A} 6,1, \mathrm{D} 5,0 \backslash \mathrm{C}, 8, \mathrm{~B} 8,3, \mathrm{~A} 7,2, \mathrm{D} 6,0 \backslash \mathrm{C}, 8, \mathrm{~B} 9,3, \mathrm{~A} 8,2, \mathrm{D} 7,0 \backslash \mathrm{C}, 8, \mathrm{~B} 10$, $3, \mathrm{~A} 9,2$, $\mathrm{D} 8,0 \backslash \mathrm{H}, 9, \mathrm{~B} 11,8, \mathrm{~A} 10,3, \mathrm{D} 9,0 \backslash \mathrm{H}, 9, \mathrm{~B} 12,8, \mathrm{~A} 11,12, \mathrm{D} 10,0 \backslash \mathrm{H}, 9, \mathrm{~B} 13,8, \mathrm{~A} 12$, 
$12, \mathrm{D} 11,0 \backslash \mathrm{H}, 10, \mathrm{~B} 14,8, \mathrm{~A} 13,3, \mathrm{D} 12,0 \backslash \mathrm{H}, 10, \mathrm{~B} 15,8, \mathrm{~A} 14,15, \mathrm{D} 13,0 \backslash \mathrm{H}, 10, \mathrm{~B} 16,8, \mathrm{~A} 15$ $, 15, \mathrm{D} 14,0 \backslash \mathrm{X}, 11,1 ., 8,90.3, \mathrm{D} 15,0 \backslash \mathrm{N}, 11, \mathrm{~B} 17,18, \mathrm{~A} 16,8,180 ., 0 \backslash \backslash \mathrm{D} 5=-2.820948$ $49 \backslash D 6=-172.62404781 \backslash D 7=68.00817564 \backslash D 8=-56.90651482 \backslash B 1=1.63879425 \backslash B 2=1$. $76680407 \backslash \mathrm{B} 3=1.51664757 \backslash \mathrm{B} 4=1.09173812 \backslash \mathrm{B} 5=1.09729372 \backslash \mathrm{B} 6=1.09598333 \backslash \mathrm{B} 7=1$. $87433331 \backslash \mathrm{B} 8=1.54722314 \backslash \mathrm{B} 9=1.53898597 \backslash \mathrm{B} 10=1.46987509 \backslash \mathrm{B} 11=1.09456438 \backslash \mathrm{B} 12$ $=1.09322437 \backslash \mathrm{B} 13=1.09446459 \backslash \mathrm{B} 14=1.09409064 \backslash \mathrm{B} 15=1.09027307 \backslash \mathrm{B} 16=1.0958685$ $8 \backslash \mathrm{B} 17=1.16134755 \backslash \mathrm{A} 1=127.88351466 \backslash \mathrm{A} 2=122.84831019 \backslash \mathrm{A} 3=109.71857276 \backslash \mathrm{A} 4=10$ $8.90699869 \backslash A 5=112.61761734 \backslash A 6=109.27146747 \backslash A 7=103.67051966 \backslash A 8=112.0180$ $1241 \backslash A 9=110.13118858 \backslash A 10=110.03666299 \backslash A 11=111.60473945 \backslash A 12=109.0263257$ $8 \backslash A 13=109.37095779 \backslash A 14=111.39024386 \backslash A 15=109.21696336 \backslash A 16=85.4520307 \backslash D 1$ $=182.8180857 \backslash \mathrm{D} 2=-29.5384385 \backslash \mathrm{D} 3=118.24218125 \backslash \mathrm{D} 4=-122.31452243 \backslash \mathrm{D} 9=-60.96$ $814176 \backslash \mathrm{D} 10=121.21780839 \backslash \mathrm{D} 11=-118.90528497 \backslash \mathrm{D} 12=57.82202549 \backslash \mathrm{D} 13=-121.128$ $30858 \backslash \mathrm{D} 14=118.88269266 \backslash \mathrm{D} 15=-130.36793124 \backslash \backslash$ Version=DEC-AXP-OSF $/ 1-\mathrm{G} 98 \mathrm{ReV}$ A. $11.3 \backslash \mathrm{HF}=-1085.1615945 \backslash \mathrm{RMSD}=2.504 \mathrm{e}-09 \backslash \mathrm{RMSF}=4.958 \mathrm{e}-05 \backslash \mathrm{Dipole}=0.2137102$ $, 1.1141232,1.4101622 \backslash \mathrm{PG}=\mathrm{C} 01 \quad \mathrm{X}(\mathrm{C} 6 \mathrm{H} 9 \mathrm{~N} 1 \mathrm{~S} 2)] \backslash \backslash @$

\section{$\mathrm{CH}_{3} \mathrm{SC} \cdot\left(\mathrm{CH}_{3}\right) \mathrm{S}-\mathrm{CH}_{2} \mathrm{Ph}(18)$}

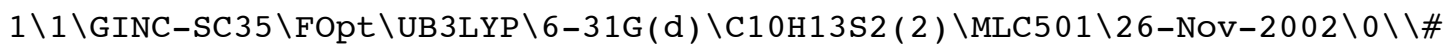
$\mathrm{N}$ UB3LYP/6-31G(D) OPT $=(\mathrm{MAXCYC}=100)$ FREQ MAXDISK $=131072000$ GUESS=READ G EOM=CHECK $\backslash \backslash$ RAFT-RAD $(\mathrm{Z}=\mathrm{Me} \mathrm{R}=\mathrm{Bz}) \quad \mathrm{c} 1 \mathrm{cy} \backslash \backslash 0,2 \backslash \mathrm{C}, 0.5508217904,1.7349275973,0$ $.082764896 \backslash \mathrm{C}, 0.56372646,1.7473599479,1.5871934348 \backslash \mathrm{S}, 2.1301757851,1.717$ $3441418,-0.6950929289 \backslash \mathrm{C}, 1.7948574291,2.2440328176,-2.4132160553 \backslash \mathrm{S},-0.9$ $429428737,1.4302432185,-0.7838339056 \backslash \mathrm{C},-0.8526164377,-0.3899166038,-1$. $2702362419 \backslash \mathrm{H}, 0.0428795461,-0.5253130123,-1.8826001106 \backslash \mathrm{H},-1.7299201211$, $-0.5085772565,-1.9145364482 \backslash \mathrm{C},-0.875490546,-1.3501477238,-0.1161572952$ $\backslash \mathrm{C},-2.0833512206,-1.7022349815,0.5041883734 \backslash \mathrm{C}, 0.3160949475,-1.90654556$ $57,0.3697099505 \backslash \mathrm{C},-2.1004443834,-2.5879588179,1.5797548248 \backslash \mathrm{C}, 0.3015747$ $185,-2.7911897776,1.4498907745 \backslash \mathrm{C},-0.9065670386,-3.1349590939,2.0577562$ $329 \backslash \mathrm{H},-3.0147042772,-1.2737279948,0.1408925506 \backslash \mathrm{H}, 1.2593017662,-1.64381$ $76211,-0.1034607552 \backslash \mathrm{H},-3.0460141015,-2.8536537482,2.0452370028 \backslash \mathrm{H}, 1.318$ $5665671,3.2267422653,-2.4245626833 \backslash \mathrm{H}, 1.1643996451,1.5261239453,-2.9404$ $378065 \backslash \mathrm{H},-0.9197307406,-3.8264159381,2.8960029584 \backslash \mathrm{H}, 1.2346124547,-3.21$ $48198333,1.8123905538 \backslash \mathrm{H},-0.3169489145,2.2771850403,1.9681460829 \backslash \mathrm{H}, 1.46$ $03717508,2.2435428602,1.9759037853 \backslash \mathrm{H}, 0.5291283588,0.7289034058,2.00268$ $91135 \backslash \mathrm{H}, 2.7706971684,2.3022233323,-2.9027282589 \backslash \backslash$ Version=DEC $-\mathrm{AXP}-\mathrm{OSF} / 1$ -G98RevA. $11.3 \backslash \mathrm{HF}=-1185.2273621 \backslash \mathrm{S} 2=0.755783 \backslash \mathrm{S} 2-1=0 . \backslash \mathrm{S} 2 \mathrm{~A}=0.750026 \backslash \mathrm{RMSD}=6$ $.979 e-09 \backslash \mathrm{RMSF}=3.861 \mathrm{e}-06 \backslash \mathrm{Dipole}=0.0663036,-0.3509731,-0.2514008 \backslash \mathrm{PG}=\mathrm{C} 01$ $[\mathrm{X}(\mathrm{C} 10 \mathrm{H} 13 \mathrm{~S} 2)] \backslash \backslash @$

\section{$\mathrm{CH}_{3} \mathrm{SCH}\left(\mathrm{CH}_{3}\right) \mathrm{S}-\mathrm{CH}_{2} \mathrm{Ph}$}

$1 \backslash 1 \backslash G I N C-S C 6 \backslash F O p t \backslash R B 3 L Y P \backslash 6-31 G(d) \backslash C 10 H 14 S 2 \backslash M L C 501 \backslash 16-J a n-2003 \backslash 0 \backslash \backslash \# \mathrm{~N}$ B3

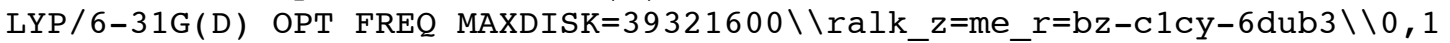
$\backslash C,-0.098469324,-0.1951139548,2.0170183918 \backslash \mathrm{C}, 1.36 \overline{5} 2311745,0.0721888135$ $, 1.6551998884 \backslash \mathrm{S},-0.9241734182,1.4223920797,2.3375427727 \backslash \mathrm{C},-2.484733035$ $2,0.8668714852,3.1046071871 \backslash \mathrm{S},-0.9857562872,-1.3112559785,0.8338540263$ $\backslash \mathrm{C},-1.1205156944,-0.3050712701,-0.7065133954 \backslash \mathrm{H},-1.448546973,0.69798418$ $29,-0.4160866759 \backslash \mathrm{H},-1.9561810874,-0.7777223648,-1.2357784874 \backslash \mathrm{C}, 0.09906$ $3464,-0.2374538504,-1.5999467068 \backslash \mathrm{C}, 0.7275286895,-1.3999358051,-2.06816$ $26983 \backslash \mathrm{C}, 0.592064356,1.0052522868,-2.0174084878 \backslash \mathrm{C}, 1.8188799725,-1.32073$ $07048,-2.9313301108 \backslash \mathrm{C}, 1.6868106491,1.0877475618,-2.8814594645 \backslash \mathrm{C}, 2.3034$ $589851,-0.0754936444,-3.3412552187 \backslash \mathrm{H}, 0.3623212923,-2.3708942297,-1.743$ $2577482 \backslash \mathrm{H}, 0.1134666594,1.9159326057,-1.6639967742 \backslash \mathrm{H}, 2.2933406977,-2.23$ $24859585,-3.285090806 \backslash \mathrm{H},-2.3016545364,0.3837013705,4.0692675754 \backslash \mathrm{H},-3.0$ $150411451,0.1792995,2.440845785 \backslash \mathrm{H}, 3.1555220602,-0.0146949833,-4.013016$ $1317 \backslash \mathrm{H}, 2.0558429711,2.0615796289,-3.192728055 \backslash \mathrm{H}, 1.8845386786,-0.874553$ $3248,1.4805800231 \backslash \mathrm{H}, 1.8634081376,0.6035868178,2.4738893892 \backslash \mathrm{H}, 1.4526323$ $98,0.6758464165,0.7481413566 \backslash \mathrm{H},-3.0943708197,1.7599805111,3.264868028 \backslash$ 
$\mathrm{H},-0.14231847,-0.7753032966,2.9455174275 \backslash \backslash$ Version=DEC-AXP-OSF $/ 1-G 98 \mathrm{ReV}$ $\mathrm{A} .11 .3 \backslash \mathrm{HF}=-1185.8759854 \backslash \mathrm{RMSD}=6.183 \mathrm{e}-09 \backslash \mathrm{RMSF}=5.091 \mathrm{e}-06 \backslash \mathrm{Dipole}=-0.014391$ $1,0.0022582,-0.026026 \backslash \mathrm{PG}=\mathrm{C} 01[\mathrm{X}(\mathrm{C} 10 \mathrm{H} 14 \mathrm{~S} 2)] \backslash \backslash @$

\section{$\mathrm{S}=\mathrm{C}\left(\mathrm{CH}_{3}\right) \mathrm{S}-\mathrm{CH}_{2} \mathrm{Ph}$}

$1 \backslash 1 \backslash G I N C-S C 97 \backslash F O p t \backslash R B 3 L Y P \backslash 6-31 G(d) \backslash C 9 H 10 S 2 \backslash M L C 501 \backslash 01-A u g-2002 \backslash 1 \backslash \backslash \# N$ B3 LYP / 6-31G* OPT $=(Z-M A T R I X, M A X C Y C=100) \quad S C F=(T I G H T, M A X C Y C=100)$ FREQ MAXDI $\mathrm{SK}=131072000 \backslash \backslash \mathrm{RAFT}(\mathrm{Z}=\mathrm{Me} \mathrm{R}=\mathrm{Bz}) \quad(\mathrm{c} 1 \mathrm{~b}) / / \mathrm{B} 3 \backslash \backslash 0,1 \backslash \mathrm{C} \backslash \mathrm{C}, 1, \mathrm{~B} 1 \backslash \mathrm{S}, 2, \mathrm{~B} 2,1, \mathrm{~A} 1 \backslash \mathrm{S}, 2$, $\mathrm{B} 3,1, \mathrm{~A} 2,3, \mathrm{D} 1,0 \backslash \mathrm{H}, 1, \mathrm{~B} 4,2, \mathrm{~A} 3,3, \mathrm{D} 2,0 \backslash \mathrm{H}, 1, \mathrm{~B} 5,2, \mathrm{~A} 4,5, \mathrm{D} 3,0 \backslash \mathrm{H}, 1, \mathrm{~B} 6,2, \mathrm{~A} 5,5, \mathrm{D} 4$, $0 \backslash \mathrm{C}, 4, \mathrm{~B} 7,2, \mathrm{~A} 6,1, \mathrm{D} 5,0 \backslash \mathrm{H}, 8, \mathrm{~B} 8,4, \mathrm{~A} 7,2, \mathrm{D} 6,0 \backslash \mathrm{H}, 8, \mathrm{~B} 9,4, \mathrm{~A} 8,2, \mathrm{D} 7,0 \backslash \mathrm{C}, 8, \mathrm{~B} 10,4, \mathrm{~A}$ 9,2 , $\mathrm{D} 8,0 \backslash \mathrm{C}, 11, \mathrm{~B} 11,8, \mathrm{~A} 10,4, \mathrm{D} 9,0 \backslash \mathrm{C}, 12, \mathrm{~B} 12,11, \mathrm{~A} 11,8, \mathrm{D} 10,0 \backslash \mathrm{C}, 13, \mathrm{~B} 13,12, \mathrm{~A} 12$

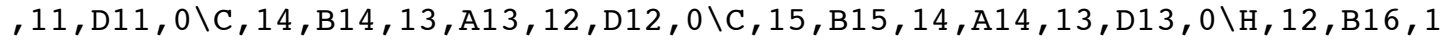
$1, \mathrm{~A} 15,13, \mathrm{D} 14,0 \backslash \mathrm{H}, 13, \mathrm{~B} 17,12, \mathrm{~A} 16,14, \mathrm{D} 15,0 \backslash \mathrm{H}, 14, \mathrm{~B} 18,13, \mathrm{~A} 17,15, \mathrm{D} 16,0 \backslash \mathrm{H}, 15$, $\mathrm{B} 19,14, \mathrm{~A} 18,16, \mathrm{D} 17,0 \backslash \mathrm{H}, 16, \mathrm{~B} 20,15, \mathrm{~A} 19,14, \mathrm{D} 18,0 \backslash \backslash \mathrm{B} 1=1.5157017 \backslash \mathrm{B} 2=1.647716$ $37 \backslash \mathrm{B} 3=1.75389067 \backslash \mathrm{B} 4=1.09284038 \backslash \mathrm{B} 5=1.0966942 \backslash \mathrm{B} 6=1.09541288 \backslash \mathrm{B} 7=1.8457545$ $\backslash \mathrm{B} 8=1.0931606 \backslash \mathrm{B} 9=1.09316751 \backslash \mathrm{B} 10=1.50746347 \backslash \mathrm{B} 11=1.40121331 \backslash \mathrm{B} 12=1.394897$ $19 \backslash \mathrm{B} 13=1.39611654 \backslash \mathrm{B} 14=1.39623894 \backslash \mathrm{B} 15=1.39469152 \backslash \mathrm{B} 16=1.0875463 \backslash \mathrm{B} 17=1.08$ $666122 \backslash \mathrm{B} 18=1.08661577 \backslash \mathrm{B} 19=1.08665644 \backslash \mathrm{B} 20=1.08753726 \backslash \mathrm{A} 1=122.61338998 \backslash \mathrm{A} 2$ $=111.38063085 \backslash A 3=109.7490219 \backslash A 4=108.97753356 \backslash A 5=112.74245768 \backslash A 6=103.93$ $454389 \backslash A 7=107.17960794 \backslash A 8=107.35823942 \backslash A 9=109.69738626 \backslash A 10=120.6321987$ $\backslash A 11=120.62629464 \backslash A 12=120.08811792 \backslash A 13=119.7336831 \backslash A 14=120.08376027 \backslash A 1$ $5=119.47797368 \backslash \mathrm{A} 16=119.7770135 \backslash \mathrm{A} 17=120.13175524 \backslash \mathrm{A} 18=120.13385522 \backslash \mathrm{A} 19=1$ $19.90004087 \backslash D 1=178.73317096 \backslash D 2=40.71288545 \backslash D 3=-117.59620812 \backslash D 4=122.324$ $46394 \backslash D 5=-177.58137358 \backslash D 6=-56.38412839 \backslash D 8=-178.59900137 \backslash D 7=59.07784813$ $\backslash \mathrm{D} 9=-90.47341043 \backslash \mathrm{D} 10=-179.84643742 \backslash \mathrm{D} 11=-0.13726456 \backslash \mathrm{D} 12=0.01725165 \backslash \mathrm{D} 13=$ $-0.01747664 \backslash D 14=-180.28619998 \backslash D 15=-180.1759319 \backslash D 16=179.82246162 \backslash D 17=17$ $9.82557756 \backslash \mathrm{D} 18=179.86971903 \backslash \backslash$ Version=DEC-AXP-OSF $/ 1-G 98 \mathrm{ReVA} .11 .3 \backslash \mathrm{HF}=-11$ $45.3573094 \backslash \mathrm{RMSD}=5.445 \mathrm{e}-09 \backslash \mathrm{RMSF}=1.591 \mathrm{e}-05 \backslash \mathrm{Dipole}=-0.9605393,0.0492055,0$ $.0284694 \backslash \mathrm{PG}=\mathrm{C} 01 \quad[\mathrm{X}(\mathrm{C} 9 \mathrm{H} 10 \mathrm{~S} 2)] \backslash \backslash @$

\section{$\mathrm{CH}_{3} \mathrm{SC} \cdot\left(\mathrm{CH}_{3}\right) \mathrm{S}-\mathrm{CH}(\mathrm{Ph}) \mathrm{CH}_{3}(19)$}

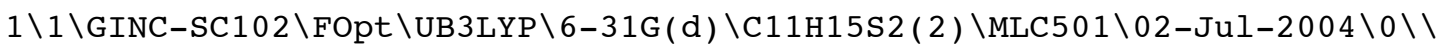
\# B3LYP/6-31G* OPT MAXDISK $=402653184 \backslash \backslash$ RRAD_Z=CH3_R=1PhEt-a1b2c1-6dub3 \ $\backslash 0,2 \backslash \mathrm{C},-1.2176028819,-1.6843968962,-0.3913 \overline{2} 91999 \backslash \mathrm{S},-1.9093817162,-1.45$ $69399145,1.210340452 \backslash \mathrm{S}, 0.492000055,-1.7258102067,-0.7274888115 \backslash \mathrm{C},-2.22$ $70532069,-1.6816464488,-1.5075943313 \backslash \mathrm{C},-0.4856943054,-1.455898616,2.34$ $72188923 \backslash \mathrm{C}, 0.905563721,-0.0301559236,-1.4995679164 \backslash \mathrm{H}, 0.1684905209,0.11$ $44142062,-2.2948715988 \backslash \mathrm{C}, 2.2939197209,-0.1870189968,-2.1290697854 \backslash \mathrm{C}, 0$. $7901810611,1.1014563651,-0.5086570492 \backslash \mathrm{C},-0.268738682,2.0138033376,-0.6$ $09791689 \backslash \mathrm{C}, 1.7234478311,1.2794405377,0.5262090162 \backslash \mathrm{H},-1.001739103,1.892$ $935148,-1.4043876911 \backslash \mathrm{H}, 2.5475627385,0.5794491486,0.6344684159 \backslash \mathrm{C},-0.393$ $5815157,3.0763941887,0.2874748765 \backslash \mathrm{C}, 1.6032366883,2.3404981666,1.421944$ $1074 \backslash \mathrm{H},-1.2233340838,3.7712310984,0.1878411758 \backslash \mathrm{H}, 2.3390809309,2.463207$ $5975,2.2126288562 \backslash \mathrm{C}, 0.5433928057,3.2446716206,1.3061911109 \backslash \mathrm{H}, 0.4508520$ $574,4.0712806966,2.0054491189 \backslash \mathrm{H}, 2.2879901725,-0.9765257367,-2.88681598$ $16 \backslash \mathrm{H}, 2.5957253405,0.752610391,-2.6054219417 \backslash \mathrm{H}, 3.0532948084,-0.44291187$ $94,-1.3825937077 \backslash \mathrm{H},-2.3272882621,-0.6892222734,-1.9757620291 \backslash \mathrm{H},-3.2232$ $911537,-1.9691594413,-1.149896426 \backslash \mathrm{H},-1.9290035856,-2.385655656,-2.2925$ $617271 \backslash \mathrm{H},-0.908950459,-1.3353182463,3.348187309 \backslash \mathrm{H}, 0.0595783422,-2.4005$ $274628,2.2923542788 \backslash \mathrm{H}, 0.1867108979,-0.62468966,2.127587509 \backslash \backslash$ Version=DE C-AXP-OSF / 1-G03RevB.03 \State $=2-A \backslash H F=-1224.5411627 \backslash \mathrm{S} 2=0.7558 \backslash \mathrm{S} 2-1=0 . \backslash \mathrm{S} 2$ $A=0.750026 \backslash R M S D=5.360 e-09 \backslash R M S F=2.368 e-06 \backslash D i p o l e=0.2447162,0.5788032,-0$ $.0008352 \backslash \mathrm{PG}=\mathrm{C} 01 \quad[\mathrm{X}(\mathrm{C} 11 \mathrm{H} 15 \mathrm{~S} 2)] \backslash \backslash @$

\section{$\mathrm{CH}_{3} \mathrm{SCH}\left(\mathrm{CH}_{3}\right) \mathrm{S}-\mathrm{CH}(\mathrm{Ph}) \mathrm{CH}_{3}$}

$1 \backslash 1 \backslash G I N C-S C 100 \backslash F O p t \backslash R B 3 L Y P \backslash 6-31 G(d) \backslash C 11 H 16 S 2 \backslash M L C 501 \backslash 14-J u 1-2004 \backslash 0 \backslash \backslash \# B$ 3LYP $/ 6-31 \mathrm{G} *$ OPT $=(\mathrm{MAXCYC}=200)$ FREQ SCF=TIGHT MAXDISK=402653184 GEOM=CHE 
CK GUESS $=$ READ $\backslash \backslash$ RALK_Z $=$ CH3 R $=1$ PhEt $-a 1 b 2 c 1-6$ dub3 $\backslash \backslash 0,1 \backslash C,-2.5183968291,-0$ $.2926052681,0.43317 \overline{3} 53 \backslash \mathrm{S},-2.569754898,1.2826324149,-0.5288147594 \backslash \mathrm{S},-0$. $8524090579,-0.7764505576,1.0766409993 \backslash \mathrm{C},-3.2164822542,-1.3716582146,-0$ $.3983420286 \backslash \mathrm{C},-1.9329603854,2.4705379341,0.7018441299 \backslash \mathrm{C}, 0.1712125276,-$ $0.9736770738,-0.4745596109 \backslash \mathrm{H},-0.3670642405,-0.3994681095,-1.2359047611$ $\backslash \mathrm{C}, 0.2716009975,-2.4418189161,-0.9115593374 \backslash \mathrm{C}, 1.5247511898,-0.32157127$ $3,-0.2613554916 \backslash \mathrm{C}, 1.8374595408,0.8750695826,-0.91980451 \backslash \mathrm{C}, 2.4882650519$ $,-0.8898120795,0.5846321821 \backslash \mathrm{H}, 1.0988509191,1.3299660889,-1.5758429179 \backslash$ $\mathrm{H}, 2$.267950214,-1.8144753528, $1.1119451657 \backslash \mathrm{C}, 3.079357983,1.4868062486,-0$ $.7434925219 \backslash \mathrm{C}, 3.728467957,-0.2780212993,0.7664037654 \backslash \mathrm{H}, 3.303492012,2.4$ $125095618,-1.2673893777 \backslash \mathrm{H}, 4.4608826697,-0.7326474325,1.4284847038 \backslash \mathrm{C}, 4$. $0299448769,0.9119950364,0.1010947041 \backslash \mathrm{H}, 4.9976152668,1.3866204587,0.240$ $2025016 \backslash \mathrm{H},-0.718362934,-2.8622250461,-1.1095960229 \backslash \mathrm{H}, 0.8719910713,-2.5$ $195227582,-1.8265634363 \backslash \mathrm{H}, 0.7456995765,-3.0594350859,-0.1425645027 \backslash \mathrm{H},-$ $2.7083879921,-1.5327789402,-1.3545213181 \backslash \mathrm{H},-4.2497383039,-1.0763657772$ $,-0.6129519155 \backslash \mathrm{H},-3.2269502263,-2.3186844507,0.1491775088 \backslash \mathrm{H},-1.8936341$ $007,3.4442556722,0.2063745529 \backslash \mathrm{H},-2.5993515673,2.5392352662,1.567048143$ $5 \backslash \mathrm{H},-0.9297554372,2.1848492216,1.027362844 \backslash \mathrm{H},-3.0779375673,-0.13221109$ $76,1.3613101272 \backslash \backslash$ Version=DEC-AXP-OSF / 1-G03RevB . 03 \State $=1-\mathrm{A} \backslash \mathrm{HF}=-1225.1$ $881185 \backslash \mathrm{RMSD}=2.075 e-09 \backslash \mathrm{RMSF}=1.961 e-06 \backslash \mathrm{Dipole}=0.0470648,-0.0040787,-0.03$ $13731 \backslash \mathrm{PG}=\mathrm{C} 01 \quad[\mathrm{X}(\mathrm{C} 11 \mathrm{H} 16 \mathrm{~S} 2)] \backslash \backslash @$

\section{$\mathrm{S}=\mathrm{C}\left(\mathrm{CH}_{3}\right) \mathrm{S}-\mathrm{CH}(\mathrm{Ph}) \mathrm{CH}_{3}$}

$1 \backslash 1 \backslash G I N C-S C 29 \backslash F O p t \backslash R B 3 L Y P \backslash 6-31 G(d) \backslash C 10 H 12 S 2 \backslash M L C 501 \backslash 02-J u 1-2004 \backslash 0 \backslash \backslash \#$ B3

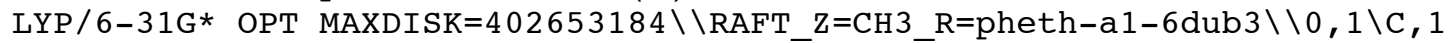
$.8274382095,-0.249707204,-3.4578286492 \backslash \mathrm{C}, 1.7 \overline{9} 17778162,-0.2747141709,-1$ $.9424462533 \backslash \mathrm{S}, 3.1664685743,-0.2797568442,-1.032255372 \backslash \mathrm{S}, 0.1373911524,-$ $0.3164708104,-1.3573337192 \backslash \mathrm{C}, 0.2728618406,-0.4524373246,0.4952594765 \backslash \mathrm{H}$ $, 1.1611040652,0.136770583,0.7434983761 \backslash \mathrm{C}, 0.5028798889,-1.9060119556,0$. $9232986023 \backslash \mathrm{C},-0.9574496829,0.1939021194,1.1006126398 \backslash \mathrm{C},-0.8458325629,1$ $.4287593708,1.7531850757 \backslash \mathrm{C},-2.2185439336,-0.4158394684,1.0295205741 \backslash \mathrm{H}$, $0.1250445823,1.9149526317,1.8131121953 \backslash \mathrm{H},-2.3302261248,-1.3716936081,0$ $.5245851886 \backslash \mathrm{C},-1.9619211313,2.0368780464,2.3284292459 \backslash \mathrm{C},-3.3369021244$, $0.1931287125,1.5987095931 \backslash \mathrm{H},-1.8541576512,2.9921440027,2.8351987099 \backslash \mathrm{H}$, $-4.3067504973,-0.2927080655,1.5324689698 \backslash \mathrm{C},-3.2117326284,1.4205218081$, $2.2521264584 \backslash \mathrm{H},-4.0825524227,1.893010618,2.6984358184 \backslash \mathrm{H}, 1.4253339621$, $2.2919967447,0.4812660697 \backslash \mathrm{H},-0.3263638869,-2.5547998566,0.6257607256 \backslash \mathrm{H}$ $, 0.5985006455,-1.9519201973,2.0144714623 \backslash \mathrm{H}, 2.6457062085,0.3892935096,-$ $3.7983705421 \backslash \mathrm{H}, 0.8849625937,0.094603437,-3.8972091512 \backslash \mathrm{H}, 2.0221887496,-$ $1.2648934371,-3.8249929431 \backslash \backslash$ Version=DEC-AXP-OSF / 1-G03RevB . 03 \State=1-A $\backslash \mathrm{HF}=-1184.6701658 \backslash \mathrm{RMSD}=5.512 \mathrm{e}-09 \backslash \mathrm{RMSF}=1.322 \mathrm{e}-06 \backslash \mathrm{Dipole}=-0.9119129,-0.0$ $367753,0.0409486 \backslash \mathrm{PG}=\mathrm{C} 01 \quad[\mathrm{X}(\mathrm{C} 10 \mathrm{H} 12 \mathrm{~S} 2)] \backslash \backslash @$

\section{$\mathrm{CH}_{3} \mathrm{SC} \cdot\left(\mathrm{CH}_{3}\right) \mathrm{S}-\mathrm{C}(\mathrm{Ph})\left(\mathrm{CH}_{3}\right)_{2}(20)$}

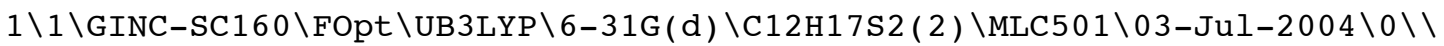

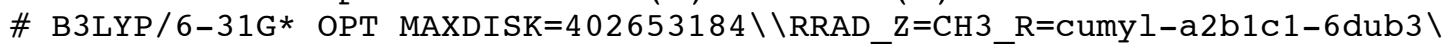
$\backslash 0,2 \backslash S,-0.7628524491,-1.2971839506,-2.9011 \overline{1} 65436 \backslash \mathrm{C},-0.7609963136,-1.27$ $03856948,-1.1372513449 \backslash \mathrm{S}, 0.6697574984,-1.4847300919,-0.1650731362 \backslash \mathrm{C},-2$ $.089733426,-0.9017572069,-0.5312818688 \backslash \mathrm{C}, 0.8612300309,-1.9807864649,-3$ $.368182635 \backslash \mathrm{C}, 1.2946597608,0.2719088123,0.359805165 \backslash \mathrm{C}, 1.6655293204,1.04$ $80335395,-0.9068197654 \backslash \mathrm{C}, 2.5708895557,-0.0445550805,1.1613234649 \backslash \mathrm{C}, 0.2$ $56795598,0.9683180919,1.2348574442 \backslash \mathrm{C},-0.2901611371,2.2143412637,0.8949$ $467456 \backslash \mathrm{C},-0.1672408748,0.3771723814,2.4387969246 \backslash \mathrm{H}, 0.0056315575,2.7087$ $022422,-0.0237138193 \backslash \mathrm{H}, 0.2157105452,-0.5998811096,2.7176946421 \backslash \mathrm{C},-1.22$ $09811186,2.8473646623,1.724377549 \backslash \mathrm{C},-1.0912742617,1.0062878448,3.26832$ $36995 \backslash \mathrm{H},-1.6266789317,3.8127233564,1.432601424 \backslash \mathrm{H},-1.4001300009,0.52273$ $46745,4.1915814897 \backslash \mathrm{C},-1.6252226329,2.2486104916,2.9150881084 \backslash \mathrm{H},-2.3485$ 
$529916,2.7396299541,3.5604953541 \backslash \mathrm{H}, 3.2989578374,-0.5744220121,0.537825$ $6373 \backslash \mathrm{H}, 3.0267394686,0.8924412995,1.5034040502 \backslash \mathrm{H}, 2.366490215,-0.6582555$ $113,2.043009512 \backslash \mathrm{H}, 2.4295062665,0.5003556057,-1.4662402173 \backslash \mathrm{H}, 2.08025262$ $07,2.0316548996,-0.650744128 \backslash \mathrm{H}, 0.8053237197,1.1988773794,-1.5658151427$ $\backslash \mathrm{H},-2.2052476002,-1.3869544428,0.4433642738 \backslash \mathrm{H},-2.9208377553,-1.2140573$ $754,-1.1756217891 \backslash \mathrm{H},-2.1854207888,0.1808771702,-0.3649881256 \backslash \mathrm{H}, 0.85454$ $18182,-2.0395329587,-4.4599884474 \backslash \mathrm{H}, 1.6746622,-1.3318599913,-3.0394893$ $937 \backslash \mathrm{H}, 0.9976040247,-2.9797243428,-2.9482413655 \backslash \backslash$ Version=DEC-AXP-OSF / 1G03RevB.03 \State $=2-\mathrm{A} \backslash \mathrm{HF}=-1263.8510387 \backslash \mathrm{S} 2=0.756265 \backslash \mathrm{S} 2-1=0 . \backslash \mathrm{S} 2 \mathrm{~A}=0.750029$ $\backslash \mathrm{RMSD}=6.961 \mathrm{e}-09 \backslash \mathrm{RMSF}=1.837 \mathrm{e}-06 \backslash \mathrm{Dipole}=0.351457,0.422136,0.0442288 \backslash \mathrm{PG}=\mathrm{C}$ $01[\mathrm{X}(\mathrm{C} 12 \mathrm{H} 17 \mathrm{~S} 2)] \backslash \backslash @$

\section{$\mathrm{CH}_{3} \mathrm{SCH}\left(\mathrm{CH}_{3}\right) \mathrm{S}-\mathrm{C}(\mathrm{Ph})\left(\mathrm{CH}_{3}\right)_{2}$}

$1 \backslash 1 \backslash G I N C-S C 37 \backslash F O p t \backslash R B 3 L Y P \backslash 6-31 G(d) \backslash C 12 H 18 S 2 \backslash M L C 501 \backslash 08-J u 1-2004 \backslash 0 \backslash \backslash \#$ B3 LYP $/ 6-31 \mathrm{G}$ * OPT $=($ TIGHT, MAXCYC=200) FREQ SCF=TIGHT MAXDISK=402653184 GEO

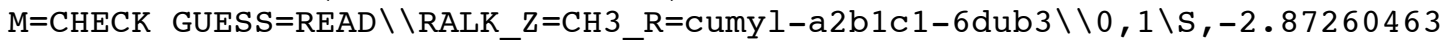
$59,-1.0748033094,0.6287894715 \backslash \bar{C},-1.6656966149,-0.907511568,-0.76351222$ $36 \backslash \mathrm{S},-0.9934760779,0.784535188,-1.1027818401 \backslash \mathrm{C},-0.6287696324,-2.024954$ $6199,-0.628226065 \backslash \mathrm{C},-4.1732347509,0.0893993565,0.0996537335 \backslash \mathrm{C}, 0.270684$ $5817,1.2770322136,0.2094153133 \backslash \mathrm{C},-0.3869613798,1.3484920648,1.59525573$ $87 \backslash \mathrm{C}, 0.6460217043,2.7140355884,-0.2254551847 \backslash \mathrm{C}, 1.5255675627,0.39552018$ $74,0.1654511075 \backslash \mathrm{C}, 2.0573641028,-0.1982199315,1.3186679572 \backslash \mathrm{C}, 2.21585860$ $54,0.2060959641,-1.0445252656 \backslash \mathrm{H}, 1.560226422,-0.077804188,2.2747444237 \backslash$ $\mathrm{H}, 1.8169678424,0.6377013819,-1.9577270592 \backslash \mathrm{C}, 3.2303273552,-0.9578136097$ $, 1.2659019617 \backslash \mathrm{C}, 3.3861737646,-0.5464695799,-1.0995387525 \backslash \mathrm{H}, 3.615507605$ $5,-1.4090416165,2.1767467849 \backslash \mathrm{H}, 3.8962253271,-0.6768175757,-2.050632701$ $1 \backslash \mathrm{C}, 3.9009402688,-1.1355582371,0.0584052981 \backslash \mathrm{H}, 4.8122844909,-1.72593924$ $46,0.0163643884 \backslash \mathrm{H},-0.2312029137,3.3691739123,-0.203724273 \backslash \mathrm{H}, 1.39443423$ $34,3.1123666687,0.4694165235 \backslash \mathrm{H}, 1.0732922233,2.7416431979,-1.2318121352$ $\backslash \mathrm{H},-1.2736541945,1.9863263289,1.5499033405 \backslash \mathrm{H}, 0.3092045465,1.7893682232$ , $2.3201969206 \backslash \mathrm{H},-0.7033666506,0.3704897973,1.9632271697 \backslash \mathrm{H}, 0.08708102,-$ $1.9796203845,-1.4533142882 \backslash \mathrm{H},-1.1333217391,-2.9972516445,-0.6489409851$ $\backslash \mathrm{H},-0.0678574604,-1.9502108854,0.3064223256 \backslash \mathrm{H},-4.9268530799,0.10272239$ $27,0.8915383116 \backslash \mathrm{H},-3.7623809958,1.0943114874,-0.0273452896 \backslash \mathrm{H},-4.644722$ $1412,-0.2375589156,-0.8326211102 \backslash \mathrm{H},-2.2342265206,-1.0658559638,-1.6875$ $261624 \backslash \backslash$ Version=DEC-AXP-OSF / 1-G03RevB.03 \State $=1-A \backslash H F=-1264.4946697 \backslash \mathrm{RM}$ $\mathrm{SD}=5.713 \mathrm{e}-09 \backslash \mathrm{RMSF}=2.010 \mathrm{e}-06 \backslash \mathrm{Dipole}=0.2260459,0.134619,-0.0155497 \backslash \mathrm{PG}=\mathrm{C} 0$ $1[\mathrm{X}(\mathrm{C} 12 \mathrm{H} 18 \mathrm{~S} 2)] \backslash \backslash @$

\section{$\mathrm{S}=\mathrm{C}\left(\mathrm{CH}_{3}\right) \mathrm{S}-\mathrm{C}(\mathrm{Ph})\left(\mathrm{CH}_{3}\right)_{2}$}

$1 \backslash 1 \backslash G I N C-S C 9 \backslash F O p t \backslash R B 3 L Y P \backslash 6-31 G(d) \backslash C 11 H 14 S 2 \backslash M L C 501 \backslash 02-J u 1-2004 \backslash 0 \backslash \backslash \#$ B3L

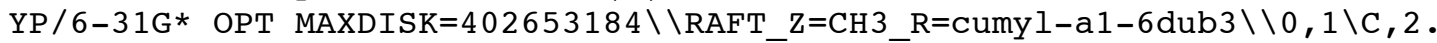
$554810971,0.0628373755,0.8708730422 \backslash \mathrm{S}, 2.532 \overline{1} 430928,0.0222117331,2.5201$ $613305 \backslash \mathrm{C}, 3.8709954546,0.084378493,0.1097040788 \backslash \mathrm{S}, 1.2273435686,0.051885$ $898,-0.2775877982 \backslash \mathrm{C},-0.4648646262,-0.0951025635,0.5756625861 \backslash \mathrm{C},-0.5222$ $140475,-1.4125977057,1.3582759289 \backslash \mathrm{C},-0.7135138848,1.0988791502,1.51520$ $68975 \backslash C,-1.4293078299,-0.0503419923,-0.6198999832 \backslash C,-2.2730699383,-1.1$ $241705446,-0.9362111961 \backslash \mathrm{C},-1.5011780694,1.1002980874,-1.4247790797 \backslash \mathrm{H},-$ $2.2489200588,-2.0319935767,-0.3446168338 \backslash \mathrm{H},-0.8512079128,1.9451864879$, $-1.2169275859 \backslash \mathrm{C},-3.1654246666,-1.046997441,-2.0080151514 \backslash \mathrm{C},-2.38599910$ $55,1.1770144048,-2.4982322564 \backslash \mathrm{H},-3.8117990476,-1.8929393824,-2.2264719$ $379 \backslash \mathrm{H},-2.4180604441,2.0787848291,-3.1038290052 \backslash \mathrm{C},-3.2263217365,0.10217$ $75667,-2.7939182692 \backslash \mathrm{H},-3.9190648903,0.1605045996,-3.629021903 \backslash \mathrm{H},-0.005$ $4041505,1.0943987361,2.3460106326 \backslash \mathrm{H}, 0.2089153908,-1.3995238871,2.16839$ $19651 \backslash \mathrm{H},-1.5176263205,-1.5339730513,1.8025819389 \backslash \mathrm{H},-1.729849172,1.0175$ $164062,1.9198353558 \backslash \mathrm{H},-0.3177118747,-2.2768025657,0.7199945394 \backslash \mathrm{H},-0.63$ $52241075,2.0556541957,0.9931375354 \backslash \mathrm{H}, 4.5866388969,0.7219259673,0.63449$ 
$36944 \backslash \mathrm{H}, 4.2823033939,-0.9320691716,0.0871732224 \backslash \mathrm{H}, 3.7617485886,0.42951$ $93325,-0.9239277201 \backslash \backslash$ Version=DEC-AXP-OSF $/ 1-G 03$ RevB . 03 \State $=1-\mathrm{A} \backslash \mathrm{HF}=-12$ 23.9747021 $\backslash \mathrm{RMSD}=6.846 \mathrm{e}-09 \backslash \mathrm{RMSF}=2.778 \mathrm{e}-06 \backslash \mathrm{Dipole}=-0.6225142,-0.0340942$, $-0.8279907 \backslash \mathrm{PG}=\mathrm{C} 01 \quad[\mathrm{X}(\mathrm{C} 11 \mathrm{H} 14 \mathrm{~S} 2)] \backslash \backslash @$

\section{$\mathrm{CH}_{3} \mathrm{SC} \cdot\left(\mathrm{CH}_{3}\right) \mathrm{S}-\mathrm{CH}_{2} \mathrm{COOCH}_{3}(21)$}

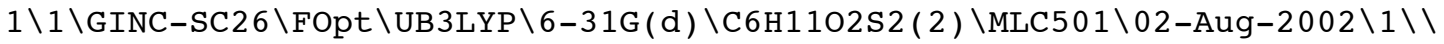
\# UB3LYP/6-31G* OPT=Z-MATRIX SCF=(QC,MAXCYC=100, TIGHT) FREQ MAXDISK=13 $1072000 \backslash \backslash$ RAFT_RAD ( $\mathrm{Z}=\mathrm{Me} \mathrm{R}=\mathrm{MA}) \mathrm{C} 1 \mathrm{p} / / \mathrm{B} 3 \backslash \backslash 0,2 \backslash \mathrm{S} \backslash \mathrm{C}, 1, \mathrm{~B} 1 \backslash \mathrm{C}, 2, \mathrm{~B} 2,1, \mathrm{~A} 1 \backslash \mathrm{S}, 2, \mathrm{~B} 3$, $1, \mathrm{~A} 2,3, \mathrm{D} 1,0 \backslash \mathrm{H}, 3, \mathrm{~B} 4,2, \mathrm{~A} 3,1, \mathrm{D} 2,0 \backslash \mathrm{H}, 3, \mathrm{~B} 5,2, \mathrm{~A} 4,5, \mathrm{D} 3,0 \backslash \mathrm{H}, 3, \mathrm{~B} 6,2, \mathrm{~A} 5,5, \mathrm{D} 4,0 \backslash \mathrm{C}$ $, 1, \mathrm{~B} 7,2, \mathrm{~A} 6,3, \mathrm{D} 5,0 \backslash \mathrm{C}, 4, \mathrm{~B} 8,2, \mathrm{~A} 7,3, \mathrm{D} 6,0 \backslash \mathrm{H}, 9, \mathrm{~B} 9,4, \mathrm{~A} 8,2, \mathrm{D} 7,0 \backslash \mathrm{H}, 9, \mathrm{~B} 10,4, \mathrm{~A} 9,2$ , $\mathrm{D} 8,0 \backslash \mathrm{C}, 9$, $\mathrm{B} 11,4, \mathrm{~A} 10,2$, D9 , 0\0 , $12, \mathrm{~B} 12,9, \mathrm{~A} 11,4, \mathrm{D} 10,0 \backslash 0,12, \mathrm{~B} 13,9, \mathrm{~A} 12,13$, D1 $1,0 \backslash \mathrm{C}, 14, \mathrm{~B} 14,12, \mathrm{~A} 13,13, \mathrm{D} 12,0 \backslash \mathrm{H}, 8, \mathrm{~B} 15,1, \mathrm{~A} 14,2, \mathrm{D} 13,0 \backslash \mathrm{H}, 8, \mathrm{~B} 16,1, \mathrm{~A} 15,16, \mathrm{D} 1$ $4,0 \backslash \mathrm{H}, 8, \mathrm{~B} 17,1, \mathrm{~A} 16,16, \mathrm{D} 15,0 \backslash \mathrm{H}, 15, \mathrm{~B} 18,14, \mathrm{~A} 17,12, \mathrm{D} 16,0 \backslash \mathrm{H}, 15, \mathrm{~B} 19,14, \mathrm{~A} 18,19$ , D17,0\H , 15, B20,14,A19, $19, \mathrm{D} 18,0 \backslash \backslash \mathrm{D} 5=-47.66210571 \backslash \mathrm{D} 6=124.2034009 \backslash \mathrm{D} 7=51$. $45345692 \backslash D 8=169.97852761 \backslash D 9=-69.03688301 \backslash D 10=79.59632628 \backslash D 11=-178.3012$ $6539 \backslash D 12=-1.10736436 \backslash D 13=-176.1569452 \backslash D 14=-118.70056952 \backslash D 15=118.684277$ $86 \backslash \mathrm{D} 16=59.84254391 \backslash \mathrm{D} 17=-120.7098879 \backslash \mathrm{D} 18=119.71257159 \backslash \mathrm{B} 1=1.75470101 \backslash \mathrm{B} 2=$ $1.50144142 \backslash \mathrm{B} 3=1.7540458 \backslash \mathrm{B} 4=1.09580114 \backslash \mathrm{B} 5=1.101524 \backslash \mathrm{B} 6=1.09607085 \backslash \mathrm{B} 7=1.8$ $3909477 \backslash \mathrm{B} 8=1.85893744 \backslash \mathrm{B} 9=1.09152394 \backslash \mathrm{B} 10=1.09162639 \backslash \mathrm{B} 11=1.50894962 \backslash \mathrm{B} 12=$ $1.21538556 \backslash \mathrm{B} 13=1.34868612 \backslash \mathrm{B} 14=1.43745151 \backslash \mathrm{B} 15=1.09346144 \backslash \mathrm{B} 16=1.09284121$ $\backslash \mathrm{B} 17=1.09195971 \backslash \mathrm{B} 18=1.0931971 \backslash \mathrm{B} 19=1.09330407 \backslash \mathrm{B} 20=1.09019523 \backslash \mathrm{A} 1=122.104$ $41177 \backslash \mathrm{A} 2=118.7219121 \backslash \mathrm{A} 3=111.19302428 \backslash \mathrm{A} 4=111.34102025 \backslash \mathrm{A} 5=111.01263129 \backslash \mathrm{A}$ $6=102.5326864 \backslash A 7=103.45710004 \backslash A 8=108.16559651 \backslash A 9=105.69370446 \backslash A 10=110$. $77782324 \backslash A 11=124.84467967 \backslash A 12=111.49500109 \backslash A 13=115.39415873 \backslash A 14=106.25$ $208603 \backslash A 15=111.51685978 \backslash A 16=109.15493098 \backslash A 17=110.56758888 \backslash A 18=110.4339$ $1008 \backslash A 19=105.51868249 \backslash D 1=179.45105199 \backslash D 2=-31.67767788 \backslash D 3=120.39887003 \backslash$ D $4=-120.58711712 \backslash \backslash$ Version=DEC-AXP-OSF / 1-G98RevA. $11.3 \backslash \mathrm{HF}=-1182.0481894 \backslash$ $\mathrm{S} 2=0.756239 \backslash \mathrm{S} 2-1=0 . \backslash \mathrm{S} 2 \mathrm{~A}=0.750029 \backslash \mathrm{RMSD}=0.000 \mathrm{e}+00 \backslash \mathrm{RMSF}=9.546 \mathrm{e}-05 \backslash \mathrm{Dipole}=$ $0.1388335,0.8127896,0.3170917 \backslash \mathrm{PG}=\mathrm{C} 01 \quad[\mathrm{X}(\mathrm{C} 6 \mathrm{H} 1102 \mathrm{~S} 2)] \backslash \backslash @$

\section{$\mathrm{CH}_{3} \mathrm{SCH}\left(\mathrm{CH}_{3}\right) \mathrm{S}-\mathrm{CH}_{2} \mathrm{COOCH}_{3}$}

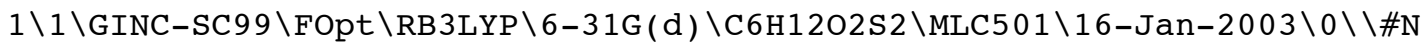
B3LYP/6-31G(D) OPT FREQ MAXDISK=39321600\\ralk_z=me $r=m a-c 1 p-6 d u b 3 \backslash \backslash 0$, $1 \backslash \mathrm{S},-2.0997683627,0.9755983623,-0.883102629 \backslash \mathrm{C},-0.26 \overline{2} 9409913,1.20626538$ $86,-0.9107020321 \backslash \mathrm{C}, 0.1627309408,2.385076654,-1.7882723541 \backslash \mathrm{S}, 0.62129505$ $91,-0.2933816099,-1.5306066769 \backslash \mathrm{H},-0.147698331,2.2372725776,-2.82745387$ $27 \backslash \mathrm{H},-0.3051313626,3.3052065946,-1.4249666316 \backslash \mathrm{H}, 1.2512092811,2.5137187$ $413,-1.7639891476 \backslash \mathrm{C},-2.4644441444,1.5467482991,0.8200002467 \backslash \mathrm{C}, 0.122693$ $1473,-1.5416017102,-0.2782295673 \backslash \mathrm{H},-0.9664962373,-1.5163531756,-0.1828$ $477314 \backslash \mathrm{H}, 0.4461799048,-2.5081803672,-0.6689336645 \backslash \mathrm{C}, 0.7569005321,-1.25$ $72168395,1.0641991048 \backslash 0,0.3562242967,-0.4238190927,1.8552508967 \backslash 0,1.84$ $61962671,-2.0194446637,1.2741391292 \backslash \mathrm{C}, 2.5507188327,-1.768290117,2.5027$ $785393 \backslash \mathrm{H},-3.5269511465,1.3548229499,0.9930824563 \backslash \mathrm{H},-2.278745775,2.6185$ $466328,0.9342036865 \backslash \mathrm{H},-1.8703921485,0.9804471434,1.5415415076 \backslash \mathrm{H}, 1.8953$ $537217,-1.9345287758,3.3615891611 \backslash \mathrm{H}, 2.9176972252,-0.7388766263,2.52771$ $90424 \backslash \mathrm{H}, 3.3824637285,-2.4728265453,2.5105213379 \backslash \mathrm{H}, 0.0447695832,1.36550$ $2814,0.125118918 \backslash \backslash$ Version=DEC-AXP-OSF / 1-G98RevA. $11.3 \backslash \mathrm{HF}=-1182.6976795 \backslash$ $\mathrm{RMSD}=3.791 \mathrm{e}-09 \backslash \mathrm{RMSF}=5.060 \mathrm{e}-06 \backslash \mathrm{Dipole}=0.4780362,-0.0867756,0.7893997 \backslash \mathrm{PG}$ $=\mathrm{C} 01[\mathrm{X}(\mathrm{C} 6 \mathrm{H} 12 \mathrm{O} 2 \mathrm{~S} 2)] \backslash \backslash @$

\section{$\mathrm{S}=\mathrm{C}\left(\mathrm{CH}_{3}\right) \mathrm{S}-\mathrm{CH}_{2} \mathrm{COOCH}_{3}$}

$1 \backslash 1 \backslash G I N C-S C 38 \backslash F O p t \backslash R B 3 L Y P \backslash 6-31 G(d) \backslash C 5 H 8 O 2 S 2 \backslash M L C 501 \backslash 01-A u g-2002 \backslash 1 \backslash \backslash \# N B$ 3LYP $/ 6-31 \mathrm{G} * \mathrm{OPT}=(\mathrm{Z}-\mathrm{MATRIX}, \mathrm{MAXCYC}=100) \mathrm{SCF}=(\mathrm{TIGHT}, \mathrm{MAXCYC}=100)$ FREQ MAXD ISK $=131072000 \backslash \backslash \mathrm{RAFT}(\mathrm{Z}=\mathrm{Me} \mathrm{R}=\mathrm{MA}) \quad(\mathrm{c} 1 \mathrm{~b}) / / \mathrm{B} 3 \backslash \backslash 0,1 \backslash \mathrm{C} \backslash \mathrm{S}, 1, \mathrm{~B} 1 \backslash \mathrm{C}, 1, \mathrm{~B} 2,2, \mathrm{~A} 1 \backslash \mathrm{S}, 1$ , $\mathrm{B} 3,2, \mathrm{~A} 2,3, \mathrm{D} 1,0 \backslash \mathrm{H}, 3, \mathrm{~B} 4,1, \mathrm{~A} 3,2, \mathrm{D} 2,0 \backslash \mathrm{H}, 3, \mathrm{~B} 5,1, \mathrm{~A} 4,5, \mathrm{D} 3,0 \backslash \mathrm{H}, 3, \mathrm{~B} 6,1, \mathrm{~A} 5,5, \mathrm{D} 4$ , $0 \backslash \mathrm{C}, 4, \mathrm{~B} 7,1, \mathrm{~A} 6,2, \mathrm{D} 5,0 \backslash \mathrm{H}, 8, \mathrm{~B} 8,4, \mathrm{~A} 7,1, \mathrm{D} 6,0 \backslash \mathrm{H}, 8, \mathrm{~B} 9,4, \mathrm{~A} 8,1, \mathrm{D} 7,0 \backslash \mathrm{C}, 8, \mathrm{~B} 10,4$, 
$\mathrm{A} 9,1, \mathrm{D} 8,0 \backslash 0,11, \mathrm{~B} 11,8, \mathrm{~A} 10,4, \mathrm{D} 9,0 \backslash 0,11, \mathrm{~B} 12,8, \mathrm{~A} 11,12, \mathrm{D} 10,0 \backslash \mathrm{C}, 13, \mathrm{~B} 13,11, \mathrm{~A} 1$ $2,8, \mathrm{D} 11,0 \backslash \mathrm{H}, 14, \mathrm{~B} 14,13, \mathrm{~A} 13,11, \mathrm{D} 12,0 \backslash \mathrm{H}, 14, \mathrm{~B} 15,13, \mathrm{~A} 14,15, \mathrm{D} 13,0 \backslash \mathrm{H}, 14, \mathrm{~B} 16,1$ $3, \mathrm{~A} 15,15, \mathrm{D} 14,0 \backslash \backslash \mathrm{D} 5=0.94844174 \backslash \mathrm{D} 6=-58.07506369 \backslash \mathrm{D} 8=-179.56933226 \backslash \mathrm{D} 7=58.8$ $8551652 \backslash \mathrm{D} 9=0.05878835 \backslash \mathrm{B} 1=1.64764965 \backslash \mathrm{B} 2=1.51577468 \backslash \mathrm{B} 3=1.75399464 \backslash \mathrm{B} 4=1.0$ $9537429 \backslash \mathrm{B} 5=1.09622663 \backslash \mathrm{B} 6=1.09314218 \backslash \mathrm{B} 7=1.82281113 \backslash \mathrm{B} 8=1.09427601 \backslash \mathrm{B} 9=1.0$ $9424892 \backslash \mathrm{B} 10=1.51743154 \backslash \mathrm{B} 11=1.21071195 \backslash \mathrm{B} 12=1.34556723 \backslash \mathrm{B} 13=1.44048522 \backslash \mathrm{B} 1$ $4=1.09284572 \backslash \mathrm{B} 15=1.09286824 \backslash \mathrm{B} 16=1.08984645 \backslash \mathrm{A} 1=122.48783328 \backslash \mathrm{A} 2=126.6156$ $9031 \backslash A 3=112.87807881 \backslash A 4=109.00443803 \backslash A 5=109.63960531 \backslash A 6=102.40049134 \backslash A$ $7=109.90359199 \backslash A 8=110.01261947 \backslash A 9=109.5060228 \backslash A 10=125.78683417 \backslash A 11=109$ $.75088779 \backslash A 12=115.36010348 \backslash A 13=110.41129286 \backslash A 14=110.40521778 \backslash A 15=105.3$ $9473697 \backslash \mathrm{D} 1=-178.78732782 \backslash \mathrm{D} 2=167.28801829 \backslash \mathrm{D} 3=120.39420154 \backslash \mathrm{D} 4=-122.15103$ $052 \backslash D 10=180.00429846 \backslash D 11=-179.98706551 \backslash D 12=-60.21919628 \backslash D 13=120.770316$ $57 \backslash \mathrm{D} 14=-119.62267623 \backslash \backslash$ Version=DEC-AXP-OSF / 1-G98RevA. $11.3 \backslash \mathrm{HF}=-1142.1744$ $019 \backslash \mathrm{RMSD}=5.217 \mathrm{e}-09 \backslash \mathrm{RMSF}=1.529 \mathrm{e}-05 \backslash \mathrm{Dipole}=-0.6553583,0.0612289,-0.13796$ $73 \backslash P G=C 01 \quad[X(C 5 H 802 S 2)] \backslash \backslash @$

\section{$\mathrm{CH}_{3} \mathrm{SC} \cdot\left(\mathrm{CH}_{3}\right) \mathrm{S}-\mathrm{CH}\left(\mathrm{COOCH}_{3}\right) \mathrm{CH}_{3}(22)$}

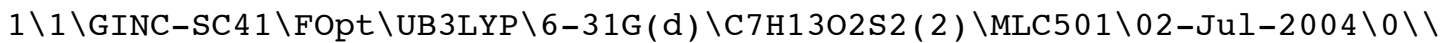

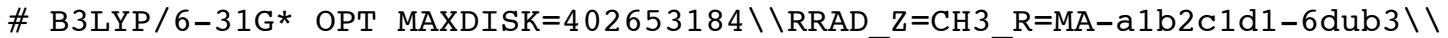
$0,2 \backslash \mathrm{S},-1.7064513367,0.4585471093,-1.5479560188 \backslash \mathrm{C},-1.2612108146,1.04526$ $09237,0.0502760557 \backslash \mathrm{S}, 0.3377633178,1.6085496057,0.4567359387 \backslash \mathrm{C},-2.30401$ $28814,0.8237776351,1.1115679166 \backslash \mathrm{C},-0.2516883218,0.7831379262,-2.601002$ $536 \backslash \mathrm{C}, 1.2006265638,0.1564804575,1.3316568904 \backslash \mathrm{H}, 0.6808602914,-0.0107128$ $881,2.2767599836 \backslash \mathrm{C}, 2.6517975984,0.5780518012,1.5496997151 \backslash \mathrm{C}, 1.08699856$ $2,-1.0778806516,0.4641796181 \backslash 0,1.7816236346,-1.3279580664,-0.499868041$ $5 \backslash 0,0.0789716746,-1.871559552,0.8866011094 \backslash \mathrm{C},-0.1738325958,-3.03429924$ $41,0.0774023692 \backslash \mathrm{H},-0.5174281462,-2.7325227938,-0.9154738363 \backslash \mathrm{H},-0.95426$ $1989,-3.5885020463,0.5992773686 \backslash \mathrm{H}, 0.7317580007,-3.6378841986,-0.019532$ $1117 \backslash \mathrm{H}, 2.701961556,1.5313433776,2.0851304809 \backslash \mathrm{H}, 3.1776135095,-0.1759524$ $768,2.1465507044 \backslash \mathrm{H}, 3.1684067131,0.6769568008,0.5917850181 \backslash \mathrm{H}, 0.61653014$ $03,0.2218835912,-2.2508230034 \backslash \mathrm{H},-0.0225612104,1.8502225602,-2.62878119$ $04 \backslash \mathrm{H},-0.5355180187,0.4441331316,-3.6010977948 \backslash \mathrm{H},-2.2929722427,1.646834$ $1297,1.8346396306 \backslash \mathrm{H},-2.1219234559,-0.1103082479,1.6644324339 \backslash \mathrm{H},-3.3102$ $879845,0.7599294787,0.6801088804 \backslash \backslash$ Version=DEC-AXP-OSF / 1-G03RevB.03 \Sta $\mathrm{te}=2-\mathrm{A} \backslash \mathrm{HF}=-1221.3644856 \backslash \mathrm{S} 2=0.755577 \backslash \mathrm{S} 2-1=0 . \backslash \mathrm{S} 2 \mathrm{~A}=0.750024 \backslash \mathrm{RMSD}=3.906 \mathrm{e}-0$ $9 \backslash \mathrm{RMSF}=8.649 \mathrm{e}-06 \backslash \mathrm{Dipole}=-0.1894324,-0.5089002,0.3515619 \backslash \mathrm{PG}=\mathrm{C} 01][\mathrm{X}(\mathrm{C} 7 \mathrm{H} 1$ $302 \mathrm{~S} 2$ ) $] \backslash \backslash$ Q

\section{$\mathrm{CH}_{3} \mathrm{SCH}\left(\mathrm{CH}_{3}\right) \mathrm{S}-\mathrm{CH}\left(\mathrm{COOCH}_{3}\right) \mathrm{CH}_{3}$}

$1 \backslash 1 \backslash G I N C-S C 51 \backslash F O p t \backslash R B 3 L Y P \backslash 6-31 G(d) \backslash C 7 H 1402 S 2 \backslash M L C 501 \backslash 14-J u 1-2004 \backslash 0 \backslash \backslash \# B$ $3 \mathrm{LYP} / 6-31 \mathrm{G} * \mathrm{OPT}=(\mathrm{MAXCYC}=200) \mathrm{FREQ} \mathrm{SCF}=\mathrm{TIGHT}$ MAXDISK=402653184 GEOM=CHE

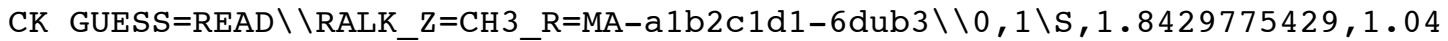
$86780294,-0.0104155 \overline{1} 5 \backslash \mathrm{C}, 1.6587111835,-0.7062188189,0.540003994 \backslash \mathrm{S}, 0.404$ $3401819,-1.7281224961,-0.349437795 \backslash \mathrm{C}, 1.4927118208,-0.7018640395,2.0615$ $341424 \backslash \mathrm{C}, 2.0249733008,0.860610607,-1.8157668893 \backslash \mathrm{C},-1.2375832607,-1.098$ $3899222,0.2168678413 \backslash \mathrm{H},-1.2962500511,-1.1897824221,1.3032038014 \backslash \mathrm{C},-2.3$ $018700511,-1.98305894,-0.449139811 \backslash \mathrm{C},-1.4606354685,0.3593824268,-0.172$ $8293177 \backslash 0,-1.5618951481,0.7712883609,-1.3073156308 \backslash 0,-1.5864066094,1.1$ $299218463,0.9284895145 \backslash \mathrm{C},-1.760718277,2.535458648,0.6781415294 \backslash \mathrm{H},-0.85$ $98378613,2.9401660964,0.2099643246 \backslash \mathrm{H},-1.9225544801,2.9887340332,1.6565$ $514776 \backslash \mathrm{H},-2.6199313674,2.7050987284,0.0247171426 \backslash \mathrm{H},-2.1403963667,-3.03$ $61976289,-0.2010598844 \backslash \mathrm{H},-3.2987626684,-1.6950094599,-0.0972704464 \backslash \mathrm{H},-$ $2.2726387123,-1.8606941885,-1.5350576149 \backslash \mathrm{H}, 1.1052536112,0.4674852975,-$ $2.2537500412 \backslash \mathrm{H}, 2.8721265857,0.2145580222,-2.0654196368 \backslash \mathrm{H}, 2.2165282173$, $1.8625643278,-2.2095299096 \backslash \mathrm{H}, 1.4331875315,-1.7278284899,2.4373502325 \backslash \mathrm{H}$ $, 0.5876072617,-0.1638610568,2.3589386647 \backslash \mathrm{H}, 2.3491524441,-0.2065379318$, $2.5323951326 \backslash \mathrm{H}, 2.582310832,-1.232785287,0.2743597121 \backslash \backslash$ Version=DEC-AXP- 
OSF $/ 1-G 03 R e v B .03 \backslash$ State $=1-A \backslash H F=-1222.0101889 \backslash$ RMSD $=4.279 e-09 \backslash \mathrm{RMSF}=1.320 \mathrm{e}$ $-05 \backslash \mathrm{Dipole}=-0.1011165,0.0093415,0.6202122 \backslash \mathrm{PG}=\mathrm{C} 01 \quad[\mathrm{X}(\mathrm{C} 7 \mathrm{H} 1402 \mathrm{~S} 2)] \backslash \backslash \mathrm{a}$

\section{$\mathrm{S}=\mathrm{C}\left(\mathrm{CH}_{3}\right) \mathrm{S}-\mathrm{CH}\left(\mathrm{COOCH}_{3}\right) \mathrm{CH}_{3}$}

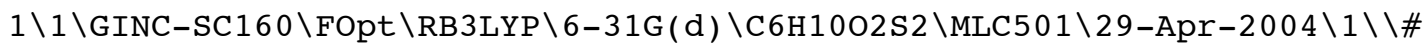
B3LYP $/ 6-31 \mathrm{G} *$ OPT $=($ MAXCYC $=300, Z-M A T R I X)$ MAXDISK $=402653184$ GEOM=CHECK GU

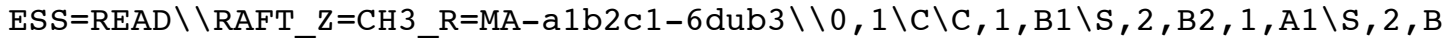
$3,1, \mathrm{~A} 2,3, \mathrm{D} 1,0 \backslash \overline{\mathrm{C}}, 4, \mathrm{~B} 4,2, \mathrm{~A} 3,1, \mathrm{D} 2,0 \backslash \mathrm{C}, 5, \mathrm{~B} 5,4, \mathrm{~A} 4,2, \mathrm{D} 3,0 \backslash 0,6, \mathrm{~B} 6,5, \mathrm{~A} 5,4, \mathrm{D} 4,0$ $\backslash 0,6, \mathrm{~B} 7,5, \mathrm{~A} 6,7, \mathrm{D} 5,0 \backslash \mathrm{C}, 8, \mathrm{~B} 8,6, \mathrm{~A} 7,5, \mathrm{D} 6,0 \backslash \mathrm{H}, 1, \mathrm{~B} 9,2, \mathrm{~A} 8,3, \mathrm{D} 7,0 \backslash \mathrm{H}, 1, \mathrm{~B} 10,2, \mathrm{~A} 9$ $, 10, \mathrm{D} 8,0 \backslash \mathrm{H}, 1, \mathrm{~B} 11,2, \mathrm{~A} 10,10, \mathrm{D} 9,0 \backslash \mathrm{C}, 5, \mathrm{~B} 12,4, \mathrm{~A} 11,6, \mathrm{D} 10,0 \backslash \mathrm{H}, 5, \mathrm{~B} 13,4, \mathrm{~A} 12,6, \mathrm{D}$ $11,0 \backslash \mathrm{H}, 13, \mathrm{~B} 14,5, \mathrm{~A} 13,4, \mathrm{D} 12,0 \backslash \mathrm{H}, 13, \mathrm{~B} 15,5, \mathrm{~A} 14,15, \mathrm{D} 13,0 \backslash \mathrm{H}, 13, \mathrm{~B} 16,5, \mathrm{~A} 15,15$, $\mathrm{D} 14,0 \backslash \mathrm{H}, 9, \mathrm{~B} 17,8, \mathrm{~A} 16,6, \mathrm{D} 15,0 \backslash \mathrm{H}, 9, \mathrm{~B} 18,8, \mathrm{~A} 17,18, \mathrm{D} 16,0 \backslash \mathrm{H}, 9, \mathrm{~B} 19,8, \mathrm{~A} 18,18, \mathrm{D} 1$ $7,0 \backslash \backslash \mathrm{B} 1=1.51550401 \backslash \mathrm{B} 2=1.64433181 \backslash \mathrm{B} 3=1.76134063 \backslash \mathrm{B} 4=1.84857026 \backslash \mathrm{B} 5=1.5251$ $7796 \backslash B 6=1.21136371 \backslash B 7=1.3462955 \backslash B 8=1.43860754 \backslash B 9=1.09526915 \backslash B 10=1.0929$ $9943 \backslash B 11=1.09643129 \backslash B 12=1.52682847 \backslash B 13=1.09514382 \backslash B 14=1.09584525 \backslash B 15=1$ $.09251016 \backslash \mathrm{B} 16=1.09306812 \backslash \mathrm{B} 17=1.09315679 \backslash \mathrm{B} 18=1.09302677 \backslash \mathrm{B} 19=1.08997545 \backslash$ $\mathrm{A} 1=122.50208778 \backslash \mathrm{A} 2=110.33614943 \backslash \mathrm{A} 3=104.50918592 \backslash \mathrm{A} 4=107.0167987 \backslash \mathrm{A} 5=124$. $676591 \backslash \mathrm{A} 6=111.0790365 \backslash \mathrm{A} 7=115.43512528 \backslash \mathrm{A} 8=112.8464058 \backslash \mathrm{A} 9=109.64544647 \backslash \mathrm{A}$ $10=108.93490901 \backslash A 11=111.82345567 \backslash A 12=104.59673725 \backslash A 13=109.68963821 \backslash A 14$ $=111.04512925 \backslash \mathrm{A} 15=110.13224412 \backslash \mathrm{A} 16=110.44524996 \backslash \mathrm{A} 17=110.45853986 \backslash \mathrm{A} 18=1$ $05.45871601 \backslash \mathrm{D} 1=181.46329095 \backslash \mathrm{D} 2=-183.21978603 \backslash \mathrm{D} 3=141.11637645 \backslash \mathrm{D} 4=99.153$ $67554 \backslash D 5=179.83858035 \backslash D 6=-181.69244048 \backslash D 7=194.84412788 \backslash D 8=122.26999047$ $\backslash \mathrm{D} 9=-120.21567462 \backslash \mathrm{D} 10=123.25466656 \backslash \mathrm{D} 11=-115.97424246 \backslash \mathrm{D} 12=178.0396616 \backslash \mathrm{D}$ $13=119.28305957 \backslash D 14=-119.25449746 \backslash D 15=-60.33898082 \backslash D 16=120.74389688 \backslash D 1$ $7=-119.61072199 \backslash \backslash$ Version=DEC-AXP-OSF $/ 1-G 03$ RevB . 03 $\backslash$ State $=1-A \backslash H F=-1181.4$ $894033 \backslash \mathrm{RMSD}=7.435 \mathrm{e}-09 \backslash \mathrm{RMSF}=9.392 \mathrm{e}-06 \backslash \mathrm{Dipole}=-0.2931424,0.4343127,-0.30$ $83545 \backslash \mathrm{PG}=\mathrm{C} 01 \quad[\mathrm{X}(\mathrm{C} 6 \mathrm{H} 10 \mathrm{O} 2 \mathrm{~S} 2)] \backslash \backslash @$

\section{$\mathrm{CH}_{3} \mathrm{SC} \cdot\left(\mathrm{CH}_{3}\right) \mathrm{S}-\mathrm{CH}_{2} \mathrm{OCOCH}{ }_{3}(23)$}

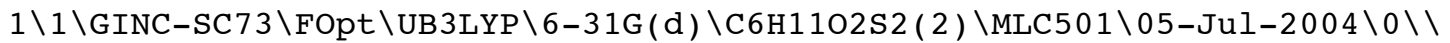

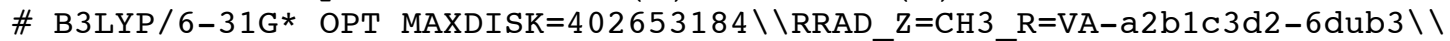
$0,2 \backslash \mathrm{S},-1.6046335589,1.301522146,0.53475091 \overline{7} 7 \backslash \mathrm{C},-\overline{1} .4065096437,-0.439314$ $5101,0.3581824035 \backslash \mathrm{S},-0.8922660445,-1.1901341949,-1.1411006036 \backslash \mathrm{C},-1.410$ $2262235,-1.2085219907,1.6498746478 \backslash \mathrm{C},-1.6293436422,1.946206834,-1.1714$ $637404 \backslash \mathrm{C}, 0.9204643955,-1.4881324727,-0.901496867 \backslash \mathrm{H}, 1.2448792839,-2.005$ $007546,-1.8074296125 \backslash \mathrm{H}, 1.1013951504,-2.0956108275,-0.0156311481 \backslash 0,1.66$ $63599954,-0.2736770225,-0.8152836802 \backslash \mathrm{C}, 2.1365151208,0.0803063968,0.416$ $6937089 \backslash 0,2.0206915597,-0.602470372,1.4092452717 \backslash \mathrm{C}, 2.7951658799,1.4353$ $600192,0.3592736658 \backslash \mathrm{H}, 2.0163081307,2.2065424717,0.3847818106 \backslash \mathrm{H}, 3.44278$ $26674,1.5604714006,1.2281279912 \backslash \mathrm{H}, 3.3624830668,1.5594511897,-0.5665777$ $672 \backslash \mathrm{H},-0.3943232028,-1.3161464413,2.0583917286 \backslash \mathrm{H},-1.8186436244,-2.2128$ $548495,1.4912150784 \backslash \mathrm{H},-2.0216995741,-0.7062569417,2.4091051623 \backslash \mathrm{H},-1.75$ $54986892,3.0281972936,-1.0776828902 \backslash \mathrm{H},-0.6929168657,1.7235331138,-1.68$ $59865348 \backslash \mathrm{H},-2.4671804492,1.5292274153,-1.7347944871 \backslash \backslash$ Version=DEC $-\mathrm{AXP}-\mathrm{O}$ $\mathrm{SF} / 1-\mathrm{G} 03 \mathrm{RevB} .03 \backslash \mathrm{State}=2-\mathrm{A} \backslash \mathrm{HF}=-1182.0520959 \backslash \mathrm{S} 2=0.755234 \backslash \mathrm{S} 2-1=0 . \backslash \mathrm{S} 2 \mathrm{~A}=0.7$ $50021 \backslash \mathrm{RMSD}=5.465 \mathrm{e}-09 \backslash \mathrm{RMSF}=4.441 \mathrm{e}-06 \backslash \mathrm{Dipole}=0.3543029,0.4721383,-0.5770$ $369 \backslash \mathrm{PG}=\mathrm{C} 01 \quad[\mathrm{X}(\mathrm{C} 6 \mathrm{H} 1102 \mathrm{~S} 2)] \backslash \backslash \mathrm{Q}$

\section{$\mathrm{CH}_{3} \mathrm{SCH}\left(\mathrm{CH}_{3}\right) \mathrm{S}-\mathrm{CH}_{2} \mathrm{OCOCH}_{3}$}

$1 \backslash 1 \backslash G I N C-S C 34 \backslash F O p t \backslash R B 3 L Y P \backslash 6-31 G(d) \backslash C 6 H 1202 S 2 \backslash M L C 501 \backslash 08-J u 1-2004 \backslash 0 \backslash \backslash \#$ B $3 \mathrm{LYP} / 6-31 \mathrm{G} * \mathrm{OPT}=(\mathrm{TIGHT}, \mathrm{MAXCYC}=300) \mathrm{FREQ}$ SCF$=\mathrm{TIGHT}$ MAXDISK $=402653184 \mathrm{GE}$

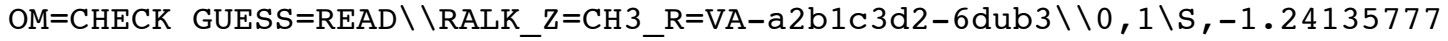
$5,1.3177160456,0.53058624 \overline{1} 2 \backslash \mathrm{C},-\overline{1} .5801478817,-0.4963758613,0.4832881406$ $\backslash \mathrm{S},-0.9160919781,-1.3980089265,-0.9946668471 \backslash \mathrm{C},-1.1727638626,-1.104996$ $7642,1.8265637805 \backslash \mathrm{C},-2.1006360405,1.8576837943,-0.9855354189 \backslash \mathrm{C}, 0.87054$ $43337,-1.5661167442,-0.6805308013 \backslash \mathrm{H}, 1.2108469472,-2.2939077218,-1.4203$ $872491 \backslash \mathrm{H}, 1.0714973016,-1.9314155874,0.3259724996 \backslash 0,1.6123802515,-0.362$ 
$7709763,-0.9132411345 \backslash C, 2.1748247813,0.2426389757,0.1691697731 \backslash 0,2.145$ $6712574,-0.2090938637,1.292874475 \backslash \mathrm{C}, 2.8118056841,1.5477706432,-0.23101$ $44283 \backslash \mathrm{H}, 2.0182152506,2.2943234899,-0.3503970307 \backslash \mathrm{H}, 3.4962197996,1.87499$ $58087,0.5527648703 \backslash \mathrm{H}, 3.3340407396,1.4547307872,-1.1868013285 \backslash \mathrm{H},-0.1112$ $577279,-0.9432207578,2.0348158453 \backslash \mathrm{H},-1.3751257323,-2.1807733911,1.8291$ $053183 \backslash \mathrm{H},-1.7508756888,-0.639551134,2.6319564156 \backslash \mathrm{H},-1.9516909149,2.937$ $8296369,-1.0653512966 \backslash \mathrm{H},-1.675102461,1.3687039937,-1.865738335 \backslash \mathrm{H},-3.17$ $44551411,1.6531051719,-0.927896788 \backslash \mathrm{H},-2.6592904794,-0.6188397441,0.338$ $5337765 \backslash \backslash$ Version=DEC-AXP-OSF $/ 1-G 03$ RevB. 03 $\backslash$ State $=1-A \backslash H F=-1182.700284 \backslash$ RM $\mathrm{SD}=2.604 \mathrm{e}-09 \backslash \mathrm{RMSF}=2.979 \mathrm{e}-06 \backslash \mathrm{Dipole}=-0.1660298,0.112638,-0.5197025 \backslash \mathrm{PG}=\mathrm{C}$ $01[\mathrm{X}(\mathrm{C} 6 \mathrm{H} 12 \mathrm{O} 2 \mathrm{~S} 2)] \backslash \backslash @$

\section{$\mathrm{S}=\mathrm{C}\left(\mathrm{CH}_{3}\right) \mathrm{S}-\mathrm{CH}_{2} \mathrm{OCOCH}$}

$1 \backslash 1 \backslash G I N C-S C 53 \backslash F O p t \backslash R B 3 L Y P \backslash 6-31 G(d) \backslash C 5 H 802 S 2 \backslash M L C 501 \backslash 02-J u 1-2004 \backslash 0 \backslash \backslash \#$ B3

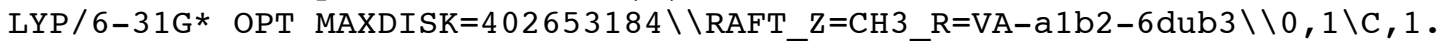
$9750593402,-0.6906704407,-2.5154211589 \backslash \mathrm{C}, 1.6 \overline{5} 01348913,-0.3029784604,-1$ $.0888895847 \backslash \mathrm{S}, 2.5261990151,-0.8251269234,0.1978201485 \backslash \mathrm{S}, 0.2070731555,0$ $.7246752909,-1.0510405555 \backslash \mathrm{C},-0.0960244756,0.9973156776,0.7317910752 \backslash \mathrm{H}$, $-0.5421795366,1.9890352195,0.814228878 \backslash \mathrm{H}, 0.8585272373,0.9210688293,1.2$ $529717273 \backslash 0,-0.9449297301,0.0155364685,1.3138958331 \backslash \mathrm{C},-2.2843842972,0$. $1892285971,1.1096864178 \backslash 0,-2.7428266425,1.1093549691,0.4732918047 \backslash \mathrm{C},-3$ $.0676524973,-0.9120390345,1.77686461 \backslash \mathrm{H},-4.1345473332,-0.7213776112,1.6$ $579717408 \backslash \mathrm{H},-2.8098780208,-1.8752537354,1.3237754242 \backslash \mathrm{H},-2.8100739776,-$ $0.97181762,2.8389061523 \backslash \mathrm{H}, 1.3996096212,-1.5869036431,-2.7809516196 \backslash \mathrm{H}, 3$ $.0364300065,-0.9284575118,-2.6072132954 \backslash \mathrm{H}, 1.7090104864,0.0966626578,-3$ $.2298517545 \backslash \backslash$ Version=DEC-AXP-OSF / 1-G03RevB.03 \State=1-A \HF=-1142.17846 $68 \backslash \mathrm{RMSD}=3.338 \mathrm{e}-09 \backslash \mathrm{RMSF}=3.386 \mathrm{e}-06 \backslash \mathrm{Dipole}=-0.2392341,-0.2456155,-0.07087$ $47 \backslash \mathrm{PG}=\mathrm{C} 01 \quad[\mathrm{X}(\mathrm{C} 5 \mathrm{H} 8 \mathrm{O} 2 \mathrm{~S} 2)] \backslash \backslash @$

\section{$\mathrm{CH}_{3} \mathrm{SC} \cdot\left(\mathrm{CH}_{3}\right) \mathrm{S}-\mathrm{CH}_{2} \mathrm{CH}_{3}(24)$}

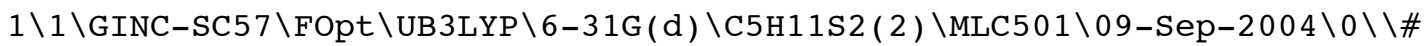

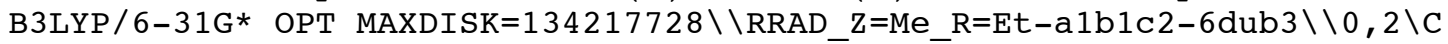
$,-0.5714512539,-0.5564439329,0.094988516 \overline{9} \backslash \mathrm{S},-\overline{0} .5650055692,-0.604787893$ $8,1.8526500644 \backslash \mathrm{S}, 0.9669930606,-0.4911502006,-0.7758965438 \backslash \mathrm{C},-1.7893355$ $415,-1.1518251078,-0.5587422291 \backslash \mathrm{C}, 0.6655047543,0.6625370943,2.33259126$ $\backslash \mathrm{C}, 0.5897960904,0.6752723494,-2.1603094186 \backslash \mathrm{H},-0.2441510744,0.273219505$ $5,-2.7446572309 \backslash \mathrm{H}, 1.4821437552,0.6213480638,-2.7933598672 \backslash \mathrm{C}, 0.31298639$ $81,2.1074427804,-1.7125615132 \backslash \mathrm{H},-2.7006113103,-0.8651638679,-0.0219773$ $509 \backslash \mathrm{H},-1.7483436946,-2.252309576,-0.5898220306 \backslash \mathrm{H},-1.8873046451,-0.8091$ $314989,-1.5953809213 \backslash \mathrm{H}, 0.6560246165,0.6890979725,3.4255499232 \backslash \mathrm{H}, 1.6639$ $613975,0.398178855,1.9816350097 \backslash \mathrm{H}, 0.3804890773,1.6415238648,1.94024960$ $01 \backslash \mathrm{H},-0.5565808062,2.1465062511,-1.0484369885 \backslash \mathrm{H}, 0.1067795092,2.7444289$ $519,-2.5819057906 \backslash \mathrm{H}, 1.1707906286,2.5254118891,-1.1757503774 \backslash \backslash$ Version=D EC-AXP-OSF / 1-G03RevB . 03 \State $=2-A \backslash H F=-993.4913813 \backslash \mathrm{S} 2=0.75518 \backslash \mathrm{S} 2-1=0 . \backslash \mathrm{S}$ $2 \mathrm{~A}=0.75002 \backslash \mathrm{RMSD}=7.019 \mathrm{e}-09 \backslash \mathrm{RMSF}=2.076 \mathrm{e}-05 \backslash \mathrm{Dipole}=-0.0510169,0.6620375,-$ $0.3780097 \backslash \mathrm{PG}=\mathrm{C} 01 \quad[\mathrm{X}(\mathrm{C} 5 \mathrm{H} 11 \mathrm{~S} 2)] \backslash \backslash \mathrm{Q}$

\section{$\mathrm{CH}_{3} \mathrm{SCH}\left(\mathrm{CH}_{3}\right) \mathrm{S}-\mathrm{CH}_{2} \mathrm{CH}_{3}$}

$1 \backslash 1 \backslash G I N C-S C 31 \backslash F O p t \backslash R B 3 L Y P \backslash 6-31 G(d) \backslash C 5 H 12 S 2 \backslash M L C 501 \backslash 10-$ Sep-2004 $0 \backslash \backslash \#$ B3L

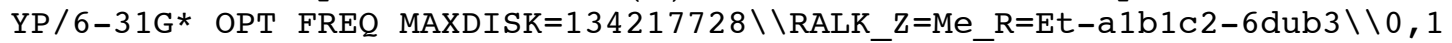
$\backslash \mathrm{C},-0.3586876963,0.6239471393,0.315666129 \backslash \overline{\mathrm{S}},-2 . \overline{0} 784229816,0.1525245159$ $,-0.122297392 \backslash \mathrm{S}, 0.8125738538,-0.0110003934,-0.9744908283 \backslash \mathrm{C},-0.29855517$ $87,2.1450539352,0.4622357811 \backslash \mathrm{C},-2.0294110335,-1.6513085187,0.159275465$ $5 \backslash \mathrm{C}, 2.4052572351,-0.015665414,-0.0466224816 \backslash \mathrm{H}, 2.5287286345,0.951483980$ $1,0.4535555453 \backslash \mathrm{H}, 3.1705064747,-0.0629463758,-0.8289005383 \backslash \mathrm{C}, 2.57385204$ $84,-1.1738407744,0.9366074497 \backslash \mathrm{H},-0.9564303827,2.4792090379,1.272686480$ $9 \backslash \mathrm{H},-0.6046708881,2.6375812349,-0.4658729939 \backslash \mathrm{H}, 0.7220371151,2.46805165$ 
$63,0.6930794754 \backslash \mathrm{H},-3.0077021726,-2.0402299956,-0.1353479653 \backslash \mathrm{H},-1.25549$ $30262,-2.1164682435,-0.4558736683 \backslash \mathrm{H},-1.8589093163,-1.8837211025,1.2150$ $110152 \backslash \mathrm{H}, 1.8131320852,-1.1519000934,1.7248086518 \backslash \mathrm{H}, 3.5558788197,-1.119$ $8855538,1.4236577718 \backslash \mathrm{H}, 2.4970768856,-2.1371201881,0.4228625879 \backslash \mathrm{H},-0.10$ $53004341,0.1424414786,1.2659710998 \backslash \backslash$ Version=DEC-AXP-OSF / 1-G03RevB.03\S tate $=1-\mathrm{A} \backslash \mathrm{HF}=-994.139074 \backslash \mathrm{RMSD}=5.500 \mathrm{e}-09 \backslash \mathrm{RMSF}=1.452 \mathrm{e}-05 \backslash \mathrm{Dipole}=0.6136177$ $,-0.2490987,0.7999153 \backslash P G=C 01[X(C 5 H 12 S 2)] \backslash \backslash @$

\section{$\mathrm{S}=\mathrm{C}\left(\mathrm{CH}_{3}\right) \mathrm{S}-\mathrm{CH}_{2} \mathrm{CH}_{3}$}

$1 \backslash 1 \backslash G I N C-S C 32 \backslash F O p t \backslash R B 3 L Y P \backslash 6-31 G(d) \backslash C 4 H 8 S 2 \backslash M L C 501 \backslash 09-S e p-2004 \backslash 0 \backslash \backslash \#$ B3LY

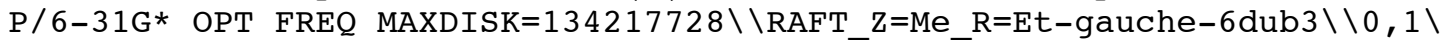
$\mathrm{C},-0.2021164069,-0.9585999653,0.258035939 \overline{9} \backslash \mathrm{S}, 1.4145758914,-1.016291554$ $5,0.5661635493 \backslash \mathrm{S},-1.1663336787,0.4841880056,-0.0245889377 \backslash \mathrm{C},-1.0554120$ $918,-2.2064011252,0.1588030295 \backslash \mathrm{C}, 0.0317386338,1.8716959479,0.025188402$ $1 \backslash \mathrm{H}, 0.7621890764,1.6304000812,0.8031614027 \backslash \mathrm{H},-0.5607800252,2.730748178$ $6,0.3558726122 \backslash \mathrm{C}, 0.7126329915,2.1422063412,-1.3151105562 \backslash \mathrm{H},-0.73052527$ $77,-2.9376778743,0.9027370235 \backslash \mathrm{H},-0.9154878523,-2.6552018848,-0.8323008$ $996 \backslash \mathrm{H},-2.1231488196,-1.9992885005,0.2892886971 \backslash \mathrm{H}, 1.3040551345,1.279272$ $2712,-1.6327838722 \backslash \mathrm{H}, 1.3896069091,2.9993255,-1.2165503967 \backslash \mathrm{H},-0.0188433$ $086,2.3726718201,-2.096119244 \backslash \backslash$ Version=DEC-AXP-OSF /1-G03RevB .03 $\backslash$ State $=$ $1-\mathrm{A} \backslash \mathrm{HF}=-953.621634 \backslash \mathrm{RMSD}=3.065 \mathrm{e}-09 \backslash \mathrm{RMSF}=8.508 \mathrm{e}-06 \backslash \mathrm{Dipole}=-0.6099368,0.4$ $058309,-0.2534361 \backslash \mathrm{PG}=\mathrm{C} 01 \quad[\mathrm{X}(\mathrm{C} 4 \mathrm{H} 8 \mathrm{~S} 2)] \backslash \backslash @$ 
Table S3. Geometries of the Leaving Group Radicals and Corresponding Alkanes, Used in Calculating the Radical Stabilization Energies in Table 3

\author{
$\cdot \mathrm{CH}_{3}(25)$ \\ $1 \backslash 1 \backslash G I N C-S C 116 \backslash F O p t \backslash U B 3 L Y P \backslash 6-31 G(d) \backslash C 1 H 3(2) \backslash R G B 501 \backslash 22-$ Aug-2002 $2 \backslash \backslash \# N B$ \\ 3LYP 6-31G(D) SCF=TIGHT OPT=TIGHT FREQ MAXDISK=13107200 \methyl Opt\&Fr \\ eq UB3-LYP $/ 6-31 \mathrm{G}(\mathrm{d}) \backslash \backslash 0,2 \backslash \mathrm{C}, 0 ., 0 ., 0 . \backslash \mathrm{H}, 1.082752351,0 ., 0 . \backslash \mathrm{H},-0.541376175$ \\ $5,-0.937691042,0 . \backslash \mathrm{H},-0.5413761755,0.937691042,0 . \backslash \backslash$ Version=DEC-AXP-OSF / \\ $1-\mathrm{G} 98 \mathrm{RevA} .11 .3 \backslash \mathrm{HF}=-39.8382922 \backslash \mathrm{S} 2=0.753765 \backslash \mathrm{S} 2-1=0 . \backslash \mathrm{S} 2 \mathrm{~A}=0.750007 \backslash \mathrm{RMSD}=4$. \\ $410 \mathrm{e}-09 \backslash \mathrm{RMSF}=1.426 \mathrm{e}-09 \backslash \mathrm{Dipole}=0 ., 0 ., 0 . \backslash \mathrm{PG}=\mathrm{D} 03 \mathrm{H} \quad[\mathrm{O}(\mathrm{C} 1), 3 \mathrm{C} 2(\mathrm{H} 1)] \backslash \backslash @$

\begin{abstract}
$\mathrm{H}-\mathrm{CH}_{3}$

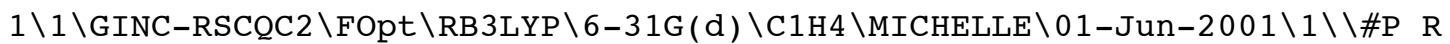
B3LYP $/ 6-31 \mathrm{G} *$ OPT=Z-MATRIX FREQ=NORAMAN MAXDISK $=262144000 \backslash \backslash$ METHANE TEST $\backslash \backslash 0,1 \backslash \mathrm{C} \backslash \mathrm{H}, 1, \mathrm{bl} \backslash \mathrm{H}, 1, \mathrm{bl}, 2,109.47122063 \backslash \mathrm{H}, 1, \mathrm{bl}, 2,109.47122063,3,120 ., 0 \backslash \mathrm{H}$, $1, \mathrm{bl}, 2,109.47122063,3,-120 ., 0 \backslash \backslash \mathrm{bl}=1.09326575 \backslash \backslash$ Version=IBM-RS6000-G98Re $\mathrm{VA} .9 \backslash \mathrm{HF}=-40.5183892 \backslash \mathrm{RMSD}=1.077 \mathrm{e}-09 \backslash \mathrm{RMSF}=1.309 \mathrm{e}-08 \backslash \mathrm{Dipole}=0 ., 0 ., 0 . \backslash \mathrm{PG}=\mathrm{T}$ D $[\mathrm{O}(\mathrm{C} 1), 4 \mathrm{C} 3(\mathrm{H} 1)] \backslash \backslash @$
\end{abstract}

\title{
$\cdot \mathrm{CH}_{2} \mathrm{CN}(26)$
}

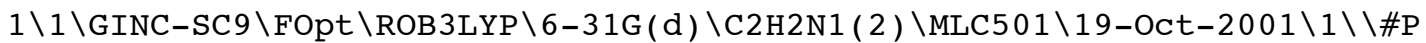
ROB3LYP/6-31G(D) FREQ OPT=Z-MATRIX GEOM=CHECK MAXDISK=1966080000 \C2V cyanomethy $1 \backslash \backslash 0,2 \backslash \mathrm{X} \backslash \mathrm{C}, 1,1 . \backslash \mathrm{N}, 2, \mathrm{CN}, 1,90 . \backslash \mathrm{C}, 2, \mathrm{CC}, 1,90 ., 3,180 ., 0 \backslash \mathrm{H}, 4, \mathrm{HC}, 2$, $\mathrm{HCC}, 1,180 ., 0 \backslash \mathrm{H}, 4, \mathrm{HC}, 2, \mathrm{HCC}, 1,0 ., 0 \backslash \backslash \mathrm{CN}=1.17367022 \backslash \mathrm{CC}=1.3865528 \backslash \mathrm{HC}=1.0836$ $2585 \backslash \mathrm{HCC}=120.29410501 \backslash \backslash$ Version=DEC-AXP-OSF $/ 1-G 98$ RevA. $9 \backslash$ State $=2-B 1 \backslash \mathrm{HF}=-$ $132.0915686 \backslash \mathrm{RMSD}=2.302 \mathrm{e}-09 \backslash \mathrm{RMSF}=1.601 \mathrm{e}-05 \backslash \mathrm{Dipole}=-1.3355252,0 ., 0 . \backslash \mathrm{PG}=\mathrm{C}$ $02 \mathrm{~V}[\mathrm{C} 2(\mathrm{C} 1 \mathrm{C} 1 \mathrm{~N} 1), \mathrm{SGV}(\mathrm{H} 2)] \backslash \backslash @$

\section{$\mathrm{H}-\mathrm{CH}_{2} \mathrm{CN}$}

$1 \backslash 1 \backslash$ GINC-SC4 $1 \backslash$ FOpt $\backslash R B 3 L Y P \backslash 6-31 G(d) \backslash C 2 H 3 N 1 \backslash R O O T \backslash 30-A u g-2001 \backslash 0 \backslash \backslash \# P$ B3LYP /6-31G(D) FREQ OPT GEOM=CHECK MAXDISK=655360000 \\C3V acetonitrile $\backslash \backslash 0,1$ $\backslash \mathrm{C}, 0 ., 0 .,-1.1813378302 \backslash \mathrm{C}, 0 ., 0 \ldots, 0.2806744874 \backslash \mathrm{N}, 0 ., 0 ., 1.4409257045 \backslash \mathrm{H}, 1.0$ $267509672,-0.0000000001,-1.5608332915 \backslash \mathrm{H},-0.5133754837,-0.8891924209,-1$ $.5608332915 \backslash \mathrm{H},-0.5133754835,0.889192421,-1.5608332915 \backslash \backslash$ Version=DEC-AXP -OSF $/ 1-G 98 R e v A .9 \backslash \mathrm{HF}=-132.7549281 \backslash \mathrm{RMSD}=5.081 \mathrm{e}-09 \backslash \mathrm{RMSF}=1.378 \mathrm{e}-04 \backslash \mathrm{Dipole}=$ $0 ., 0 .,-1.5002578 \backslash \mathrm{PG}=\mathrm{C} 03 \mathrm{~V}[\mathrm{C} 3(\mathrm{C} 1 \mathrm{C} 1 \mathrm{~N} 1), 3 \mathrm{SGV}(\mathrm{H} 1)] \backslash \backslash @$

\section{- $\mathrm{C}\left(\mathrm{CH}_{3}\right)_{2} \mathrm{CN}(27)$}

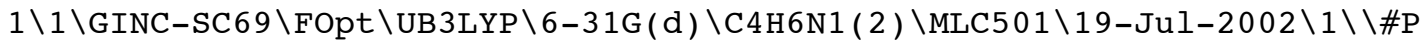
UB3LYP $/ 6-31 \mathrm{G} *$ FOPT $=(Z-M A T R I X$, TIGHT, MAXCYC $=100)$ FREQ MAXDISK $=131072000$ $\mathrm{SCF}=(\mathrm{TIGHT}, \mathrm{MAXCYC}=100) \backslash \backslash \mathrm{me} 2 \mathrm{CCn}-\mathrm{c} 2 \mathrm{vb}-6 \mathrm{dub} 3 \backslash \backslash 0,2 \backslash \mathrm{C} \backslash \mathrm{C}, 1, \mathrm{~b} 1 \backslash \mathrm{X}, 2,1 ., 1,90 . \backslash \mathrm{N}$ $, 2, \mathrm{~b} 2,3,90 ., 1,180,, 0 \backslash \mathrm{C}, 1, \mathrm{~b} 3,2, \mathrm{a} 2,3,0 ., 0 \backslash \mathrm{C}, 1, \mathrm{~b} 3,2, \mathrm{a} 2,3,180 ., 0 \backslash \mathrm{H}, 5, \mathrm{~b} 4,1$, $\mathrm{a} 3,2,0 ., 0 \backslash \mathrm{H}, 6, \mathrm{~b} 4,1, \mathrm{a} 3,2,0 ., 0 \backslash \mathrm{H}, 5, \mathrm{~b} 5,1, \mathrm{a} 4,7, \mathrm{~d} 1,0 \backslash \mathrm{H}, 5, \mathrm{~b} 5,1, \mathrm{a} 4,7,-\mathrm{d} 1,0 \backslash \mathrm{H}$, $6, \mathrm{~b} 5,1, \mathrm{a} 4,8, \mathrm{~d} 1,0 \backslash \mathrm{H}, 6, \mathrm{~b} 5,1, \mathrm{a} 4,8,-\mathrm{d} 1,0 \backslash \backslash \mathrm{b} 1=1.39173287 \backslash \mathrm{b} 2=1.17666605 \backslash \mathrm{b} 3=1$ $.50156142 \backslash \mathrm{b} 4=1.09243341 \backslash \mathrm{b} 5=1.09976071 \backslash \mathrm{a} 2=120.24203494 \backslash \mathrm{a} 3=112.14994686 \backslash$ $\mathrm{a} 4=110.62200038 \backslash \mathrm{d} 1=121.09506187 \backslash \backslash$ Version=DEC-AXP-OSF $/ 1-G 98 R e v A .11 .3 \backslash \mathrm{St}$ ate $=2-\mathrm{B} 1 \backslash \mathrm{HF}=-210.7373225 \backslash \mathrm{S} 2=0.765669 \backslash \mathrm{S} 2-1=0 . \backslash \mathrm{S} 2 \mathrm{~A}=0.750113 \backslash \mathrm{RMSD}=8.850 \mathrm{e}-$ $09 \backslash \mathrm{RMSF}=1.667 \mathrm{e}-06 \backslash \mathrm{Dipole}=0 ., 0 .,-1.623572 \backslash \mathrm{PG}=\mathrm{C} 02 \mathrm{~V} \quad[\mathrm{C} 2(\mathrm{C} 1 \mathrm{C} 1 \mathrm{~N} 1), \mathrm{SGV}(\mathrm{C} 2 \mathrm{H} 2)$ , $\mathrm{X}(\mathrm{H} 4) \mathrm{J} \backslash \backslash @$ 


\section{$\mathrm{H}-\mathrm{C}\left(\mathrm{CH}_{3}\right)_{2} \mathrm{CN}$}

$1 \backslash 1 \backslash G I N C-S C 9 \backslash F O p t \backslash R B 3 L Y P \backslash 6-31 G(d) \backslash C 4 H 7 N 1 \backslash M L C 501 \backslash 20-S e p-2002 \backslash 1 \backslash \backslash \#$ B3LYP

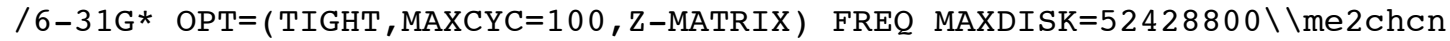
$-\mathrm{Cs}-6 \mathrm{dub} 3 \backslash \backslash 0,1 \backslash \mathrm{C} \backslash \mathrm{C}, 1, \mathrm{~B} 1 \backslash \mathrm{X}, 2,1 ., 1,90 . \backslash \mathrm{N}, 2, \mathrm{~B} 2,3, \mathrm{~A} 1,1,180 ., 0 \backslash \mathrm{C}, 1, \mathrm{~B} 3,2, \mathrm{~A} 2$, $4, \mathrm{D} 1,0 \backslash \mathrm{C}, 1, \mathrm{~B} 3,2, \mathrm{~A} 2,4,-\mathrm{D} 1,0 \backslash \mathrm{H}, 5, \mathrm{~B} 4,1, \mathrm{~A} 3,2, \mathrm{D} 2,0 \backslash \mathrm{H}, 6, \mathrm{~B} 4,1, \mathrm{~A} 3,2,-\mathrm{D} 2,0 \backslash \mathrm{H}, 5$, $\mathrm{B} 5,1, \mathrm{~A} 4,7, \mathrm{D} 3,0 \backslash \mathrm{H}, 5, \mathrm{~B} 6,1, \mathrm{~A} 5,7, \mathrm{D} 4,0 \backslash \mathrm{H}, 6, \mathrm{~B} 6,1, \mathrm{~A} 5,8,-\mathrm{D} 4,0 \backslash \mathrm{H}, 6, \mathrm{~B} 5,1, \mathrm{~A} 4,8,-\mathrm{D}$ $3,0 \backslash \mathrm{H}, 1, \mathrm{~B} 7,2, \mathrm{~A} 6,4,180 ., 0 \backslash \backslash \mathrm{B} 7=1.09858521 \backslash \mathrm{A} 6=106.60432393 \backslash \mathrm{B} 1=1.47251393 \backslash$ $\mathrm{B} 2=1.16116373 \backslash \mathrm{B} 3=1.54180066 \backslash \mathrm{B} 4=1.09489716 \backslash \mathrm{B} 5=1.09399754 \backslash \mathrm{B} 6=1.0950837 \backslash \mathrm{A}$ $1=89.39524564 \backslash \mathrm{A} 2=110.46001989 \backslash \mathrm{A} 3=110.73492571 \backslash \mathrm{A} 4=111.34414552 \backslash \mathrm{A} 5=109.5$ $9179039 \backslash D 1=62.34809296 \backslash D 2=296.77899993 \backslash D 3=120.52082383 \backslash D 4=-119.4655672$ $4 \backslash \backslash$ Version=DEC-AXP-OSF / 1-G98RevA.11.3 $\backslash$ State $=1-A^{\prime} \backslash H F=-211.3831069 \backslash$ RMSD $=$ $5.940 e-09 \backslash \mathrm{RMSF}=3.751 \mathrm{e}-06 \backslash \mathrm{Dipole}=0.0514549,0,,-1.550366 \backslash \mathrm{PG}=\mathrm{CS} \quad[\mathrm{SG}(\mathrm{C} 2 \mathrm{H} 1 \mathrm{~N}$ $1), \mathrm{X}(\mathrm{C} 2 \mathrm{H} 6) \mathrm{]} \backslash \backslash \mathrm{Q}$

\section{$-\mathrm{CH}_{2} \mathrm{Ph}(28)$}

$1 \backslash 1 \backslash G I N C-S C 89 \backslash F O p t \backslash U B 3 L Y P \backslash 6-31 G(d) \backslash C 7 H 7(2) \backslash M L C 501 \backslash 18-J u 1-2002 \backslash 1 \backslash \backslash \#$ UB3 LYP / 6-31G * FOPT $=($ TIGHT, MAXCYC $=100$, Z-MATRIX $)$ MAXDISK $=131072000$ FREQ SCF $=($ TIGHT, MAXCYC $=100) \backslash \backslash \mathrm{bz} \_r a d-c 2 v-6$ dub3 (guess from dave) $\backslash \backslash 0,2 \backslash C \backslash C, 1, B 1 \backslash$ $\mathrm{C}, 2, \mathrm{~B} 2,1, \mathrm{~A} 1 \backslash \mathrm{C}, 2, \mathrm{~B} 2,1, \mathrm{~A} 1,3,-180$, $0 \backslash \mathrm{C}, 3, \mathrm{~B} 3,2, \mathrm{~A} 2,1,-180$, $0 \backslash \mathrm{C}, 4, \mathrm{~B} 3,2, \mathrm{~A} 2,1$, $180 ., 0 \backslash \mathrm{H}, 1, \mathrm{~B} 4,2, \mathrm{~A} 3,3,0 ., 0 \backslash \mathrm{H}, 1, \mathrm{~B} 4,2, \mathrm{~A} 3,7,-180.0 \backslash \mathrm{H}, 3, \mathrm{~B} 5,2, \mathrm{~A} 4,5,180 ., 0 \backslash \mathrm{H}$ , 4 , B5 , $2, \mathrm{~A} 4,6,-180 ., 0 \backslash \mathrm{H}, 5, \mathrm{~B} 6,3, \mathrm{~A} 5,2,180.0 \backslash \mathrm{H}, 6, \mathrm{~B} 6,4, \mathrm{~A} 5,2,-180 ., 0 \backslash \mathrm{X}, 2,1$. $, 1,90 ., 3,90 ., 0 \backslash \mathrm{C}, 2, \mathrm{~B} 7,13,90.1,180 ., 0 \backslash \mathrm{X}, 14,1.2,90 ., 13,0.0 \backslash \mathrm{H}, 14, \mathrm{~B} 8,15$ $, 90 ., 2,180 ., 0 \backslash \backslash \mathrm{B} 1=1.40658309 \backslash \mathrm{B} 2=1.4269787 \backslash \mathrm{B} 3=1.38585898 \backslash \mathrm{B} 4=1.08519274 \backslash$ $\mathrm{B} 5=1.08733663 \backslash \mathrm{B} 6=1.08707607 \backslash \mathrm{B} 7=2.83514342 \backslash \mathrm{B} 8=1.08628629 \backslash \mathrm{A} 1=121.3932742$ $5 \backslash A 2=121.13892253 \backslash A 3=121.22638869 \backslash A 4=118.71171974 \backslash A 5=119.70158608 \backslash \backslash$ Ver sion=DEC-AXP-OSF $/ 1-G 98 R e v A .11 .3 \backslash$ State $=2-B 1 \backslash H F=-270.9151435 \backslash S 2=0.783683$ $\backslash \mathrm{S} 2-1=0 . \backslash \mathrm{S} 2 \mathrm{~A}=0.750769 \backslash \mathrm{RMSD}=5.840 \mathrm{e}-09 \backslash \mathrm{RMSF}=5.706 \mathrm{e}-07 \backslash \mathrm{Dipole}=0 ., 0 ., 0.052$ $5474 \backslash \mathrm{PG}=\mathrm{C} 02 \mathrm{~V} \quad[\mathrm{C} 2(\mathrm{H} 1 \mathrm{C} 1 \mathrm{C} 1 \mathrm{C} 1), \mathrm{SGV}(\mathrm{C} 4 \mathrm{H} 6)] \backslash \backslash \mathrm{Q}$

\section{$\mathrm{H}-\mathrm{CH}_{2} \mathrm{Ph}$}

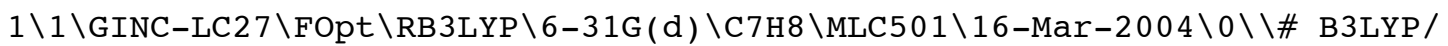
6-31G* SCF=TIGHT OPT=TIGHT FREQ MAXDISK $=402653184 \backslash \backslash \mathrm{CH} 3 \mathrm{Ph} \backslash \backslash 0,1 \backslash \mathrm{H},-0.012$ $280233,0.7346071951,2.1468279811 \backslash \mathrm{C},-0.0074819681,0.1943288714,1.202363$ $6156 \backslash \mathrm{C},-0.0067265807,-1.2011662732,1.2054029939 \backslash \mathrm{C},-0.0056582472,-1.905$ $0507128,0 . \backslash \mathrm{C},-0.0067265807,-1.2011662732,-1.2054029939 \backslash \mathrm{C},-0.0074819681$ $, 0.1943288714,-1.2023636156 \backslash \mathrm{C},-0.004493163,0.9139230304,0 . \backslash \mathrm{C}, 0.0272590$ $169,2.4252659094,0 . \backslash \mathrm{H}, 1.0589780721,2.8022373154,0 . \backslash \mathrm{H},-0.4676659209,2.8$ $373136412,0.8860302766 \backslash \mathrm{H},-0.4676659209,2.8373136412,-0.8860302766 \backslash \mathrm{H},-0$ $.012280233,0.7346071951,-2.1468279811 \backslash \mathrm{H},-0.0114411572,-1.7385214103,-2$ $.1504319414 \backslash \mathrm{H},-0.0083465047,-2.9918167074,0 . \backslash \mathrm{H},-0.0114411572,-1.738521$ $4103,2.1504319414 \backslash \backslash$ Version=x86-Linux-G03RevB .03 $\backslash$ State $=1-A^{\prime} \backslash \mathrm{HF}=-271.566$ $6505 \backslash \mathrm{RMSD}=8.405 \mathrm{e}-09 \backslash \mathrm{RMSF}=1.362 \mathrm{e}-06 \backslash \mathrm{Dipole}=0.0152484,0.1248415,0 . \backslash \mathrm{PG}=\mathrm{CS}$

$[\mathrm{SG}(\mathrm{C} 3 \mathrm{H} 2), \mathrm{X}(\mathrm{C} 4 \mathrm{H} 6)] \backslash \backslash \mathrm{Q}$

\section{$\cdot \mathrm{CH}(\mathrm{Ph}) \mathrm{CH}_{3}(29)$}

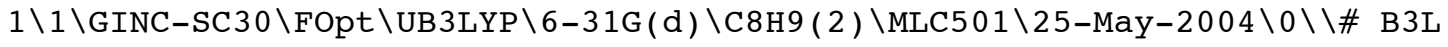

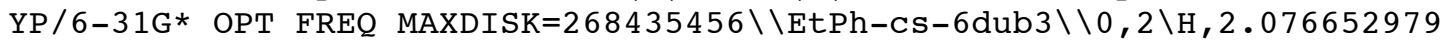
$1,-1.7071221701,0 . \backslash \mathrm{C}, 1.8330785143,-0.6472341403,0 . \backslash \mathrm{C}, 2.9648478287,0.33$ $29041964,0 . \backslash C, 0.4615044195,-0.296422334,0 . \backslash C,-2.3017509074,0.350177759$ $9,0 . \backslash \mathrm{C},-0.5414638247,-1.3098262805,0 . \backslash \mathrm{C}, 0.01601966,1.0572057614,0 . \backslash \mathrm{C},-$ $1.3371627939,1.3659918739,0 . \backslash \mathrm{C},-1.8898621213,-0.9905058968,0 . \backslash \mathrm{H},-0.229$ $1124677,-2.3517513472,0 . \backslash \mathrm{H}, 0.7487305703,1.8588289809,0 . \backslash \mathrm{H},-1.649403422$ $1,2.4074639039,0 . \backslash \mathrm{H},-2.6317608755,-1.785156805,0 . \backslash \mathrm{H},-3.3593148857,0.59$ $85933381,0 . \backslash \mathrm{H}, 3.930913811,-0.179956881,0 . \backslash \mathrm{H}, 2.94101482,0.9926776704,0$. $8806011513 \backslash \mathrm{H}, 2.94101482,0.9926776704,-0.8806011513 \backslash \backslash$ Version=DEC-AXP-OS $\mathrm{F} / 1-\mathrm{G} 03 \mathrm{RevB} .03 \backslash \mathrm{State}=2-\mathrm{A} " \backslash \mathrm{HF}=-310.2333536 \backslash \mathrm{S} 2=0.779917 \backslash \mathrm{S} 2-1=0 . \backslash \mathrm{S} 2 \mathrm{~A}=0.75$ $0613 \backslash \mathrm{RMSD}=8.432 \mathrm{e}-09 \backslash \mathrm{RMSF}=3.493 \mathrm{e}-05 \backslash \mathrm{Dipole}=0.1484688,0.0260889,0 . \backslash \mathrm{PG}=\mathrm{CS}$ 
$[\mathrm{SG}(\mathrm{C} 8 \mathrm{H} 7), \mathrm{X}(\mathrm{H} 2)] \backslash \backslash @$

\section{$\mathrm{H}-\mathrm{CH}(\mathrm{Ph}) \mathrm{CH}_{3}$}

$1 \backslash 1 \backslash$ GINC-SC122\FOpt \RB3LYP $\backslash 6-31 \mathrm{G}(\mathrm{d}) \backslash \mathrm{C} 8 \mathrm{H} 10 \backslash \mathrm{MLC} 501 \backslash 11-\mathrm{Sep}-2002 \backslash 0 \backslash \backslash \# \mathrm{P}$ B3L YP $/ 6-31 \mathrm{G}(\mathrm{D})$ FOPT $=(\mathrm{MAXCYC}=100)$ FREQ MAXDISK $=52428800$ SCF $=(\mathrm{TIGHT}, \mathrm{MAXCYC}=$ $100) \mathrm{GEOM}=\mathrm{CHECK} \backslash \backslash$ etbz-cs $-6 \mathrm{drb} 3 \backslash \backslash 0,1 \backslash \mathrm{C},-2.2284396309,1.7737516665,0 . \backslash \mathrm{C}$, $-0.6937105063,1.8926699354,0 . \backslash \mathrm{C}, 0 ., 0.546181554,0 . \backslash \mathrm{C}, 0.3118254669,-0.10$ $23160492,1.2026834225 \backslash \mathrm{C}, 0.3118254669,-0.1023160492,-1.2026834225 \backslash \mathrm{C}, 0.9$ $132220777,-1.3616103083,1.2059190433 \backslash \mathrm{C}, 0.9132220777,-1.3616103083,-1.2$ $059190433 \backslash \mathrm{C}, 1.2153222385,-1.9966792384,0 . \backslash \mathrm{H}, 1.6863498799,-2.976105526$, $0 . \backslash \mathrm{H}, 1.150067146,-1.8447129978,2.1505767223 \backslash \mathrm{H}, 1.150067146,-1.844712997$ $8,-2.1505767223 \backslash \mathrm{H}, 0.0837929653,0.387950677,2.1471919348 \backslash \mathrm{H}, 0.0837929653$ $, 0.387950677,-2.1471919348 \backslash \mathrm{H},-0.3779096411,2.4687487917,-0.8791267801 \backslash$ $\mathrm{H},-0.3779096411,2.4687487917,0.8791267801 \backslash \mathrm{H},-2.5794995785,1.2298765659$ $, 0.8842051352 \backslash \mathrm{H},-2.5794995785,1.2298765659,-0.8842051352 \backslash \mathrm{H},-2.69885480$ $66,2.7639522374,0 . \backslash \backslash V e r s i o n=D E C-A X P-O S F / 1-G 98 R e v A .11 .3 \backslash$ State $=1-A^{\prime} \backslash H F=-$ $310.8802404 \backslash \mathrm{RMSD}=5.588 \mathrm{e}-09 \backslash \mathrm{RMSF}=2.772 \mathrm{e}-06 \backslash \mathrm{Dipole}=-0.0632775,0.0900445$, $0 . \backslash P G=C S[S G(C 4 H 2), X(C 4 H 8)] \backslash \backslash @$

\section{$\cdot \mathrm{C}(\mathrm{Ph})\left(\mathrm{CH}_{3}\right)_{2}(30)$}

$1 \backslash 1 \backslash G I N C-S C 28 \backslash F O p t \backslash U B 3 L Y P \backslash 6-31 G(d) \backslash C 9 H 11(2) \backslash M L C 501 \backslash 25-M a y-2004 \backslash 0 \backslash \backslash \#$ B3

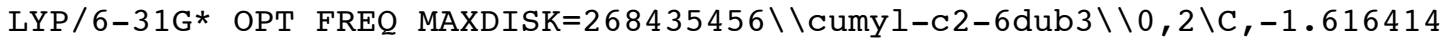
3386,0 . $0 . \backslash \mathrm{C},-0.1860957499,0.0 . \backslash \mathrm{C}, 0.5630674826,1.2120912698,0 . \backslash \mathrm{C}, 0.56$ $30674826,-1.2120912698,0 . \backslash \mathrm{C}, 1.9511243291,1.2069476466,0 . \backslash \mathrm{C}, 1.951124329$ $1,-1.2069476466,0 . \backslash \mathrm{H}, 0.0402217434,2.1628990039,0 . \backslash \mathrm{H}, 0.0402217434,-2.16$ $28990039,0 . \backslash \mathrm{H}, 2.4890058288,2.1518597904,0 . \backslash \mathrm{H}, 2.4890058288,-2.151859790$ $4,0 . \backslash \mathrm{C},-2.382682254,1.2955409885,0 . \backslash \mathrm{C},-2.382682254,-1.2955409885,0 . \backslash \mathrm{H}$, $-3.4618855224,1.1182105536,0 . \backslash \mathrm{H},-3.4618855224,-1.1182105536,0 . \backslash \mathrm{H},-2.15$ $20630813,1.9147870606,0.8799660511 \backslash \mathrm{H},-2.1520630813,-1.9147870606,0.879$ $9660511 \backslash \mathrm{H},-2.1520630813,1.9147870606,-0.8799660511 \backslash \mathrm{H},-2.1520630813,-1$. $9147870606,-0.8799660511 \backslash \mathrm{C}, 2.6605839324,0 ., 0 . \backslash \mathrm{H}, 3.7470104688,0 ., 0 . \backslash \backslash \mathrm{Ve}$ rsion=DEC-AXP-OSF $/ 1-G 03 R e v B .03 \backslash$ State $=2-B 1 \backslash H F=-349.5492998 \backslash \mathrm{S} 2=0.775843 \backslash$ $\mathrm{S} 2-1=0 . \backslash \mathrm{S} 2 \mathrm{~A}=0.750464 \backslash \mathrm{RMSD}=4.694 \mathrm{e}-09 \backslash \mathrm{RMSF}=4.133 \mathrm{e}-05 \backslash \mathrm{Dipole}=-0.2539911,0$ , $0 . \backslash \mathrm{PG}=\mathrm{C} 02 \mathrm{~V} \quad[\mathrm{C} 2(\mathrm{C} 1 \mathrm{C} 1 \mathrm{C} 1 \mathrm{H} 1), \mathrm{SGV}(\mathrm{C} 6 \mathrm{H} 6), \mathrm{X}(\mathrm{H} 4)] \backslash \backslash @$

\section{$\mathrm{H}-\mathrm{C}(\mathrm{Ph})\left(\mathrm{CH}_{3}\right)_{2}$}

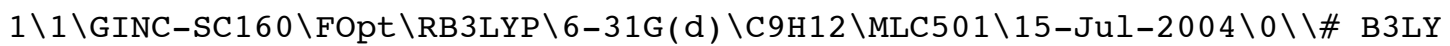

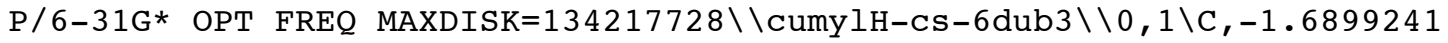
$576,-0.1717677053,0 . \backslash \mathrm{C},-0.166110256,-0.1721242299,0 . \backslash \mathrm{C}, 0.5651701726,1$. $025588545,0 . \backslash \mathrm{C}, 1.959755901,1.0169364848,0 . \backslash \mathrm{C}, 0.5469439405,-1.377640064$ $8,0 . \backslash \mathrm{C}, 1.9433393106,-1.393118293,0 . \backslash \mathrm{C}, 2.6557562567,-0.1940404185,0 . \backslash \mathrm{H}$, $0.0402648699,1.9781154329,0 . \backslash \mathrm{H}, 0.0000587161,-2.3183655685,0 . \backslash \mathrm{H}, 2.50448$ $70428,1.9578251307,0 . \backslash \mathrm{H}, 2.4725022991,-2.3427864574,0 . \backslash \mathrm{H}, 3.7425448432,-$ $0.2012622131,0 . \backslash \mathrm{H},-2.0123581199,-1.2220929864,0 . \backslash \mathrm{C},-2.2637087336,0.485$ $062341,1.2695572918 \backslash \mathrm{C},-2.2637087336,0.485062341,-1.2695572918 \backslash \mathrm{H},-1.992$ $4183132,1.5459811224,1.3260966421 \backslash \mathrm{H},-1.9924183132,1.5459811224,-1.3260$ $966421 \backslash \mathrm{H},-1.885411406,-0.0043887136,2.1735361138 \backslash \mathrm{H},-1.885411406,-0.004$ $3887136,-2.1735361138 \backslash \mathrm{H},-3.3584612085,0.4208139215,1.2778278313 \backslash \mathrm{H},-3.3$ $584612085,0.4208139215,-1.2778278313 \backslash \backslash$ Version=DEC-AXP-OSF / 1-G03RevB.03 $\backslash$ State $=1-A^{\prime} \backslash H F=-350.193313 \backslash R M S D=5.904 e-09 \backslash R M S F=3.037 e-05 \backslash D i p o l e=-0.105$ $8048,-0.0014748,0 . \backslash \mathrm{PG}=\mathrm{CS} \quad[\mathrm{SG}(\mathrm{C} 7 \mathrm{H} 6), \mathrm{X}(\mathrm{C} 2 \mathrm{H} 6)] \backslash \backslash @$

\section{- $\mathrm{CH}_{2} \mathrm{COOCH}_{3}$ (31)}

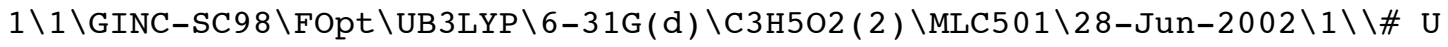
B3LYP /6-31G* FOPT=(Z-MATRIX, MAXCYC $=100$, TIGHT $)$ MAXDISK=65536000 FREQ SC $\mathrm{F}=(\mathrm{TIGHT}, \mathrm{MAXCYC}=100) \backslash \backslash \mathrm{ch} 2 \mathrm{cooch} 3 \mathrm{rad}(\mathrm{MA}) \mathrm{Cs} \backslash \backslash 0,2 \backslash \mathrm{C} \backslash \mathrm{C}, 1, \mathrm{~B} 1 \backslash 0,2, \mathrm{~B} 2,1, \mathrm{~A} 1 \backslash$ $\mathrm{O}, 2, \mathrm{~B} 3,1, \mathrm{~A} 2,3,-180 ., 0 \backslash \mathrm{C}, 4, \mathrm{~B} 4,2, \mathrm{~A} 3,1,180.0 \backslash \mathrm{H}, 5, \mathrm{~B} 5,4, \mathrm{~A} 4,2,-180 ., 0 \backslash \mathrm{H}, 1, \mathrm{~B}$ 
$6,2, \mathrm{~A} 5,3,180 ., 0 \backslash \mathrm{H}, 1, \mathrm{~B} 7,2, \mathrm{~A} 6,7,-180 ., 0 \backslash \mathrm{H}, 5, \mathrm{~B} 8,4, \mathrm{~A} 7,6, \mathrm{D} 1,0 \backslash \mathrm{H}, 5, \mathrm{~B} 8,4, \mathrm{~A} 7,6$ $,-D 1,0 \backslash \backslash \mathrm{B} 1=1.44732926 \backslash \mathrm{B} 2=1.22312018 \backslash \mathrm{B} 3=1.36100596 \backslash \mathrm{B} 4=1.43336725 \backslash \mathrm{B} 5=1.0$ $9058408 \backslash \mathrm{B} 6=1.0833186 \backslash \mathrm{B} 7=1.0824072 \backslash \mathrm{B} 8=1.09394961 \backslash \mathrm{A} 1=124.74743883 \backslash \mathrm{A} 2=111$ $.87082202 \backslash A 3=114.93235024 \backslash A 4=105.62119929 \backslash A 5=121.24789916 \backslash A 6=118.31516$ $036 \backslash A 7=110.72623732 \backslash D 1=119.68853085 \backslash \backslash$ Version=DEC-AXP-OSF $/ 1-G 98$ RevA. 11 . $3 \backslash$ State $=2-A^{\prime \prime} \backslash \mathrm{HF}=-267.7237418 \backslash \mathrm{S} 2=0.757594 \backslash \mathrm{S} 2-1=0 . \backslash \mathrm{S} 2 \mathrm{~A}=0.750034 \backslash \mathrm{RMSD}=4.6$ $63 e-09 \backslash \mathrm{RMSF}=3.942 \mathrm{e}-06 \backslash \mathrm{Dipole}=-0.6413843,0,,-0.2480326 \backslash \mathrm{PG}=\mathrm{CS} \quad[\mathrm{SG}(\mathrm{C} 3 \mathrm{H} 3 \mathrm{O} 2$ ), $\mathrm{X}(\mathrm{H} 2) \mathrm{J} \backslash \backslash \mathrm{Q}$

\section{$\mathrm{H}-\mathrm{CH}_{2} \mathrm{COOCH}_{3}$}

$1 \backslash 1 \backslash G I N C-S C 105 \backslash F O p t \backslash R B 3 L Y P \backslash 6-31 G(d) \backslash C 3 H 602 \backslash M L C 501 \backslash 16-M a r-2004 \backslash 0 \backslash \backslash \#$ B3L YP/6-31G* OPT FREQ MAXDISK $65536000 \backslash \backslash \mathrm{ch} 3 \mathrm{cooch} 3-$ geom-6dub3 $\backslash \backslash 0,1 \backslash \mathrm{C}, 0 ., 0$. , $0 . \backslash 0,1.1806641117,0.2693571148,0 . \backslash 0,-0.4766353163,-1.2676815605,0 . \backslash \mathrm{C}$, $0.5238438782,-2.2984404094,0 . \backslash \mathrm{C},-1.1338518662,0.9987105655,0 . \backslash \mathrm{H},-0.726$ $1641654,2.0099935722,0 . \backslash \mathrm{H},-0.0257634362,-3.2403316547,0 . \backslash \mathrm{H}, 1.155560253$ $1,-2.2224675233,0.88922 \backslash \mathrm{H}, 1.1555602531,-2.2224675233,-0.88922 \backslash \mathrm{H},-1.765$ $6876699,0.851506761,-0.88217 \backslash \mathrm{H},-1.7656876699,0.851506761,0.88217 \backslash \backslash$ Vers ion=DEC-AXP-OSF /1-G03RevB.03\State=1-A ' $\backslash \mathrm{HF}=-268.3884834 \backslash \mathrm{RMSD}=2.990 \mathrm{e}-09$ $\backslash \mathrm{RMSF}=3.448 \mathrm{e}-05 \backslash \mathrm{Dipole}=-0.6571523,0.2409099,0 . \backslash \mathrm{PG}=\mathrm{CS} \quad[\mathrm{SG}(\mathrm{C} 3 \mathrm{H} 2 \mathrm{O} 2), \mathrm{X}(\mathrm{H} 4)$ ]$\backslash \backslash Q$

\section{$\cdot \mathrm{CH}\left(\mathrm{COOCH}_{3}\right) \mathrm{CH}_{3}(32)$}

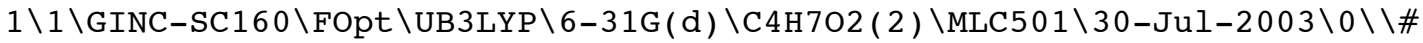
B3LYP/6-31G* OPT=TIGHT FREQ MAXDISK=39321600 GEOM=CHECK GUESS=READ \\MA rad-Csc-6dub3 (EA conf) $\backslash \backslash 0,2 \backslash C, 2.4427069032,-0.6700725424,0 . \backslash C, 0.9884$ $\overline{4} 54995,-0.9680099437,0 . \backslash \mathrm{C}, 0.0000531191,0.0925714969,0 . \backslash 0,0.2492141423$, $1.2915528696,0 . \backslash 0,-1.2690884584,-0.4038514401,0 . \backslash \mathrm{C},-2.3055213085,0.584$ $7337423,0 . \backslash \mathrm{H},-3.2434106254,0.0277541879,0 . \backslash \mathrm{H}, 2.6133119038,0.4089560571$ $, 0 . \backslash \mathrm{H}, 0.6334677625,-1.9941959598,0 . \backslash \mathrm{H}, 2.9379202621,-1.1090091687,0.878$ $9917495 \backslash \mathrm{H}, 2.9379202621,-1.1090091687,-0.8789917495 \backslash \mathrm{H},-2.2371601584,1.2$ $192780488,0.8886413064 \backslash \mathrm{H},-2.2371601584,1.2192780488,-0.8886413064 \backslash \backslash$ Ver sion=DEC-AXP-OSF / 1-G03RevB.03 \State $=2-A " \backslash H F=-307.0484404 \backslash S 2=0.756686 \backslash S$ $2-1=0 . \backslash \mathrm{S} 2 \mathrm{~A}=0.750029 \backslash \mathrm{RMSD}=9.256 \mathrm{e}-09 \backslash \mathrm{RMSF}=1.294 \mathrm{e}-06 \backslash \mathrm{Dipole}=-0.0936169,-0$ $.6405142,0 . \backslash P G=C S[S G(C 4 H 3 O 2), X(\mathrm{H} 4)] \backslash \backslash @$

\section{$\mathrm{H}-\mathrm{CH}\left(\mathrm{COOCH}_{3}\right) \mathrm{CH}_{3}$}

$1 \backslash 1 \backslash G I N C-S C 30 \backslash F O p t \backslash R B 3 L Y P \backslash 6-31 G(d) \backslash C 4 H 8 O 2 \backslash M L C 501 \backslash 05-J u 1-2004 \backslash 0 \backslash \backslash \#$ B3LY

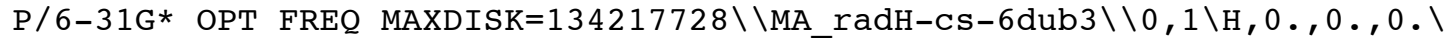
$\mathrm{C},-0.4422940416,-1.0015569368,0 . \backslash \mathrm{C}, 0.65 \overline{1} 788309,-2.0673146905,0 . \backslash \mathrm{C}, 0.09$ $63901805,-3.4787652506,0 . \backslash 0,-1.0779926594,-3.7776321358,0 . \backslash 0,1.0973279$ $219,-4.3906403923,0 . \backslash \mathrm{C}, 0.6772568456,-5.7641339686,0 . \backslash \mathrm{H}, 1.5959113266,-6$ $.3518520842,0 . \backslash \mathrm{H}, 0.0804265039,-5.984952561,0.88921 \backslash \mathrm{H}, 0.0804265039,-5.9$ $84952561,-0.88921 \backslash \mathrm{H}, 1.3092671468,-1.967050819,0.87323 \backslash \mathrm{H}, 1.3092671468,-$ $1.967050819,-0.87323 \backslash \mathrm{H},-1.0829853622,-1.0970017348,0.88164 \backslash \mathrm{H},-1.082985$ $3622,-1.0970017348,-0.88164 \backslash \backslash$ Version=DEC-AXP-OSF / 1-G03RevB .03 $\backslash$ State $=1-$ $A^{\prime} \backslash \mathrm{HF}=-307.7031831 \backslash \mathrm{RMSD}=3.847 \mathrm{e}-09 \backslash \mathrm{RMSF}=1.483 e-05 \backslash \mathrm{Dipole}=-0.2025393,0.6$ $17604,0 . \backslash P G=C S[S G(C 4 \mathrm{H} 2 \mathrm{O} 2), \mathrm{X}(\mathrm{H} 6)] \backslash \backslash @$

\section{$-\mathrm{CH}_{2} \mathrm{OCOCH}_{3}(33)$}

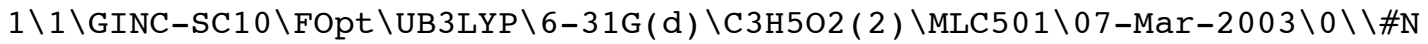

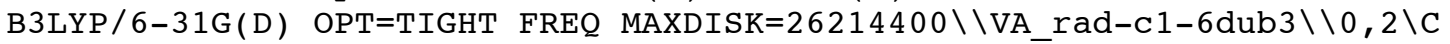
$,-1.7787811457,0.353773456,-0.0356590445 \backslash \mathrm{C},-0.2730471777,0.3716029248$, $0.0126778637 \backslash 0,0.4350619367,1.3462970692,0.0902212033 \backslash 0,0.2185047767,-$ $0.9118545746,-0.0469137652 \backslash \mathrm{H},-2.1541016217,1.3743705621,0.0441727311 \backslash \mathrm{C}$ $, 1.5760766477,-1.0845083545,-0.0455384982 \backslash \mathrm{H}, 2.202147411,-0.2279043038$, $0.1585273774 \backslash \mathrm{H}, 1.8766726719,-2.1166078394,0.0561765013 \backslash \mathrm{H},-2.1785837176$ $,-0.2564307074,0.7808932818 \backslash \mathrm{H},-2.1201583965,-0.0941758259,-0.975111322$ 
$\backslash \backslash$ Version=DEC-AXP-OSF $/ 1-G 98 R e v A .11 .3 \backslash \mathrm{HF}=-267.7202621 \backslash \mathrm{S} 2=0.753529 \backslash \mathrm{S} 2-1=$ $0 . \backslash \mathrm{S} 2 \mathrm{~A}=0.750008 \backslash \mathrm{RMSD}=8.716 \mathrm{e}-09 \backslash \mathrm{RMSF}=1.382 \mathrm{e}-06 \backslash \mathrm{Dipole}=-0.4442854,-0.574$ $2031,0.0426497 \backslash \mathrm{PG}=\mathrm{C} 01 \quad[\mathrm{X}(\mathrm{C} 3 \mathrm{H} 5 \mathrm{O} 2)] \backslash \backslash @$

\section{$\mathrm{H}-\mathrm{CH}_{2} \mathrm{OCOCH}_{3}$}

$1 \backslash 1 \backslash G I N C-S C 102 \backslash F O p t \backslash R B 3 L Y P \backslash 6-31 G(d) \backslash C 3 H 602 \backslash M L C 501 \backslash 07-J u 1-2004 \backslash 0 \backslash \backslash \#$ B3L YP/6-31G* OPT=TIGHT FREQ SCF=TIGHT MAXDISK=268435456\\VA_radH-Cs-6dub3 $\backslash \backslash 0,1 \backslash \mathrm{H},-2.0507709135,-1.6098881047,0 . \backslash \mathrm{C},-0.9603664636,-\overline{1} .6097553923,0$ $. \backslash \mathrm{C},-0.4580146239,-0.1847545269,0 . \backslash 0,-1.1494991678,0.8094818734,0 . \backslash 0,0$ $.8958438124,-0.1526126433,0 . \backslash \mathrm{C}, 1.477739726,1.1606885207,0 . \backslash \mathrm{H}, 2.5568007$ $512,1.0029959847,0 . \backslash \mathrm{H}, 1.1711784917,1.7182601627,0.8892030117 \backslash \mathrm{H}, 1.17117$ $84917,1.7182601627,-0.8892030117 \backslash \mathrm{H},-0.5876479043,-2.1408268278,0.88201$ $3079 \backslash \mathrm{H},-0.5876479043,-2.1408268278,-0.882013079 \backslash \backslash$ Version=DEC-AXP-OSF $/ 1$ -G03RevB.03 $\backslash$ State $=1-A^{\prime} \backslash \mathrm{HF}=-268.3884834 \backslash \mathrm{RMSD}=7.490 \mathrm{e}-09 \backslash \mathrm{RMSF}=2.142 \mathrm{e}-06 \backslash \mathrm{D}$ ipole $=0.5733172,-0.4020735,0 . \backslash \mathrm{PG}=\mathrm{CS} \quad[\mathrm{SG}(\mathrm{C} 3 \mathrm{H} 2 \mathrm{O} 2), \mathrm{X}(\mathrm{H} 4)] \backslash \backslash \mathrm{Q}$

\section{$\cdot \mathrm{CH}_{2} \mathrm{CH}_{3}(34)$}

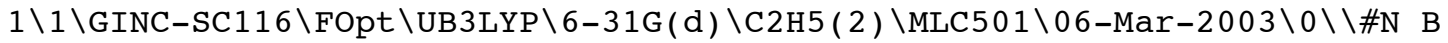

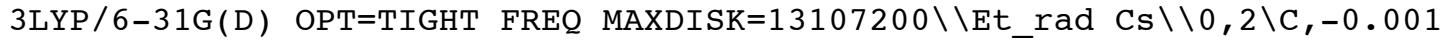
$4599287,0.7954773663,0 . \backslash \mathrm{C}, 0.0222526364,-0.6939020591,0 . \backslash \mathrm{H},-0.994810722$ $3,-1.1263631861,0 . \backslash \mathrm{H},-0.0929966331,1.3519648812,0.9274633127 \backslash \mathrm{H},-0.0929$ $966331,1.3519648812,-0.9274633127 \backslash \mathrm{H}, 0.5280238711,-1.0935092097,0.88764$ $34386 \backslash \mathrm{H}, 0.5280238711,-1.0935092097,-0.8876434386 \backslash \backslash$ Version=DEC-AXP-OSF / 1-G98RevA. 11.3\State=2-A ' $\backslash \mathrm{HF}=-79.1578673 \backslash \mathrm{S} 2=0.753921 \backslash \mathrm{S} 2-1=0 . \backslash \mathrm{S} 2 \mathrm{~A}=0.750$ $01 \backslash \mathrm{RMSD}=8.620 \mathrm{e}-09 \backslash \mathrm{RMSF}=3.177 \mathrm{e}-06 \backslash \mathrm{Dipole}=-0.0504824,-0.0860887,0 . \backslash \mathrm{PG}=\mathrm{CS}$ $[\mathrm{SG}(\mathrm{C} 2 \mathrm{H} 1), \mathrm{X}(\mathrm{H} 4)] \backslash \backslash @$

\section{$\mathrm{H}-\mathrm{CH}_{2} \mathrm{CH}_{3}$}

$1 \backslash 1 \backslash G I N C-S C 4 \backslash F O p t \backslash R B 3 L Y P \backslash 6-31 G(d) \backslash C 2 H 6 \backslash M L C 501 \backslash 29-J u n-2003 \backslash 0 \backslash \backslash \#$ B3LYP/6

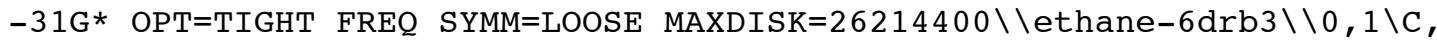
$-0.7652714924,0.0 . \backslash \mathrm{C}, 0.7652714924,0 ., 0 . \backslash \mathrm{H}, 1.1643473724,1.0209355355,0$ $.0000019949 \backslash \mathrm{H}, 1.1643473724,-0.5104694954,0.8841551119 \backslash \mathrm{H}, 1.1643473724,-$ $0.5104660401,-0.8841571069 \backslash \mathrm{H},-1.1643473724,-1.0209355355,-0.0000019949$ $\backslash \mathrm{H},-1.1643473724,0.5104660401,0.8841571069 \backslash \mathrm{H},-1.1643473724,0.510469495$ $4,-0.8841551119 \backslash \backslash$ Version=DEC-AXP-OSF $/ 1-G 03$ RevB . 03 $\backslash$ State $=1-A 1 G \backslash H F=-79.8$ $304175 \backslash \mathrm{RMSD}=4.869 \mathrm{e}-10 \backslash \mathrm{RMSF}=8.778 \mathrm{e}-07 \backslash \mathrm{Dipole}=0 ., 0 ., 0 . \backslash \mathrm{PG}=\mathrm{D} 03 \mathrm{D}[\mathrm{C} 3(\mathrm{C} 1 . \mathrm{C} 1$ ), 3SGD ( $\mathrm{H} 2)] \backslash \backslash @$ 
Table S4. Geometries of the RAFT-adduct Radicals, and Corresponding Alkanes, Used in Calculating the Radical Stabilization Energies and Beta-Scission Enthalpies

\section{in Table 4}

\section{$\mathrm{CH}_{3} \mathrm{SC} \cdot\left(\mathrm{CH}_{2} \mathrm{Ph}\right) \mathrm{S}-\mathrm{C}\left(\mathrm{CH}_{3}\right)_{2} \mathrm{CN}(35)$}

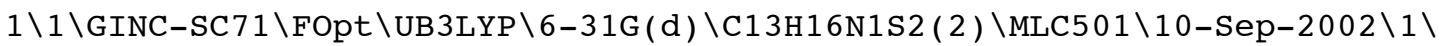
$\backslash \# \mathrm{~N}$ UB3LYP/6-31G(D) OPT=(Z-MATRIX, MAXCYC=100) FREQ MAXDISK=131072000 S $\mathrm{CF}=(\mathrm{TIGHT}, \mathrm{MAXCYC}=100)$ INT $(\mathrm{GRID}=\mathrm{ULTRAFINE}) \quad \mathrm{GEOM}=\mathrm{CHECK} \backslash \backslash \mathrm{RRAD}(\mathrm{Z}=\mathrm{Bz} \mathrm{R}=\mathrm{MCN})$ $\mathrm{C} 1 \backslash \backslash 0,2 \backslash \mathrm{C} \backslash \mathrm{S}, 1, \mathrm{~B} 1 \backslash \mathrm{S}, 1, \mathrm{~B} 2,2, \mathrm{~A} 1 \backslash \mathrm{C}, 1, \mathrm{~B} 3,2, \mathrm{~A} 2,3, \mathrm{D} 1,0 \backslash \mathrm{C}, 4, \mathrm{~B} 4,1, \mathrm{~A} 3,2, \mathrm{D} 2,0 \backslash \mathrm{H}$, $4, \mathrm{~B} 5,1, \mathrm{~A} 4,2, \mathrm{D} 3,0 \backslash \mathrm{H}, 4, \mathrm{~B} 6,1, \mathrm{~A} 5,2, \mathrm{D} 4,0 \backslash \mathrm{C}, 3, \mathrm{~B} 7,1, \mathrm{~A} 6,2, \mathrm{D} 5,0 \backslash \mathrm{C}, 2, \mathrm{~B} 8,1, \mathrm{~A} 7,3, \mathrm{D}$ $6,0 \backslash \mathrm{C}, 9, \mathrm{~B} 9,2, \mathrm{~A} 8,1, \mathrm{D} 7,0 \backslash \mathrm{C}, 9, \mathrm{~B} 10,2, \mathrm{~A} 9,1, \mathrm{D} 8,0 \backslash \mathrm{C}, 9, \mathrm{~B} 11,2, \mathrm{~A} 10,1, \mathrm{D} 9,0 \backslash \mathrm{H}, 11, \mathrm{~B}$ $12,9, \mathrm{~A} 11,2, \mathrm{D} 10,0 \backslash \mathrm{H}, 11, \mathrm{~B} 13,9, \mathrm{~A} 12,13, \mathrm{D} 11,0 \backslash \mathrm{H}, 11, \mathrm{~B} 14,9, \mathrm{~A} 13,13, \mathrm{D} 12,0 \backslash \mathrm{H}, 8, \mathrm{~B}$ $15,3, \mathrm{~A} 14,1, \mathrm{D} 13,0 \backslash \mathrm{H}, 8, \mathrm{~B} 16,3, \mathrm{~A} 15,16, \mathrm{D} 14,0 \backslash \mathrm{H}, 8, \mathrm{~B} 17,3, \mathrm{~A} 16,16, \mathrm{D} 15,0 \backslash \mathrm{C}, 5, \mathrm{~B} 18$ , $4, \mathrm{~A} 17,1, \mathrm{D} 16,0 \backslash \mathrm{C}, 19, \mathrm{~B} 19,5, \mathrm{~A} 18,4, \mathrm{D} 17,0 \backslash \mathrm{C}, 20, \mathrm{~B} 20,19, \mathrm{~A} 19,5, \mathrm{D} 18,0 \backslash \mathrm{C}, 21, \mathrm{~B} 21$ , 20 , $\mathrm{A} 20,19, \mathrm{D} 19,0 \backslash \mathrm{C}, 22, \mathrm{~B} 22,21, \mathrm{~A} 21,20, \mathrm{D} 20,0 \backslash \mathrm{H}, 23, \mathrm{~B} 23,22, \mathrm{~A} 22,21, \mathrm{D} 21,0 \backslash \mathrm{H}, 2$ 2 , B2 4, $21, \mathrm{~A} 23,23, \mathrm{D} 22,0 \backslash \mathrm{H}, 21, \mathrm{~B} 25,20, \mathrm{~A} 24,22, \mathrm{D} 23,0 \backslash \mathrm{H}, 20, \mathrm{~B} 26,19, \mathrm{~A} 25,21, \mathrm{D} 24$, $0 \backslash \mathrm{H}, 19, \mathrm{~B} 27,5, \mathrm{~A} 26,20, \mathrm{D} 25,0 \backslash \mathrm{H}, 10, \mathrm{~B} 28,9, \mathrm{~A} 27,2, \mathrm{D} 26,0 \backslash \mathrm{H}, 10, \mathrm{~B} 29,9, \mathrm{~A} 28,29, \mathrm{D} 27$ $, 0 \backslash \mathrm{H}, 10, \mathrm{~B} 30,9, \mathrm{~A} 29,29, \mathrm{D} 28,0 \backslash \mathrm{X}, 12,1 ., 9,90.2, \mathrm{D} 29,0 \backslash \mathrm{N}, 12, \mathrm{~B} 31,32, \mathrm{~A} 30,9,180$ ., $0 \backslash \backslash \mathrm{B} 1=1.74158315 \backslash \mathrm{B} 2=1.75854282 \backslash \mathrm{B} 3=1.51907585 \backslash \mathrm{B} 4=1.52268081 \backslash \mathrm{B} 5=1.0958$ $8909 \backslash B 6=1.09817176 \backslash B 7=1.82271722 \backslash B 8=1.92209999 \backslash B 9=1.53935692 \backslash B 10=1.534$ $22373 \backslash \mathrm{B} 11=1.46613269 \backslash \mathrm{B} 12=1.09437876 \backslash \mathrm{B} 13=1.09651774 \backslash \mathrm{B} 14=1.09314929 \backslash \mathrm{B} 15=$ $1.09224159 \backslash \mathrm{B} 16=1.09145599 \backslash \mathrm{B} 17=1.09324982 \backslash \mathrm{B} 18=1.39957428 \backslash \mathrm{B} 19=1.39671712$ $\backslash \mathrm{B} 20=1.39507851 \backslash \mathrm{B} 21=1.39728989 \backslash \mathrm{B} 22=1.39428744 \backslash \mathrm{B} 23=1.08779339 \backslash \mathrm{B} 24=1.087$ $11536 \backslash \mathrm{B} 25=1.0868793 \backslash \mathrm{B} 26=1.08697623 \backslash \mathrm{B} 27=1.08719939 \backslash \mathrm{B} 28=1.09372247 \backslash \mathrm{B} 29=1$ $.09542121 \backslash \mathrm{B} 30=1.09431937 \backslash \mathrm{B} 31=1.16285746 \backslash \mathrm{A} 1=123.71423017 \backslash \mathrm{A} 2=121.8675341$ $1 \backslash A 3=113.0507604 \backslash A 4=109.65077295 \backslash A 5=108.81848364 \backslash A 6=105.50539826 \backslash A 7=10$ $6.73616068 \backslash A 8=104.45253669 \backslash A 9=111.2714673 \backslash A 10=108.42077149 \backslash A 11=109.517$ $10323 \backslash A 12=110.19190686 \backslash A 13=111.40642512 \backslash A 14=110.865312 \backslash A 15=111.3486486$ $7 \backslash A 16=105.65278598 \backslash A 17=120.54947403 \backslash A 18=120.55011096 \backslash A 19=120.19384831 \backslash$ $\mathrm{A} 20=119.62352595 \backslash \mathrm{A} 21=120.11929502 \backslash \mathrm{A} 22=119.85626635 \backslash \mathrm{A} 23=120.08796574 \backslash \mathrm{A} 2$ $4=120.22372982 \backslash \mathrm{A} 25=119.67165151 \backslash \mathrm{A} 26=119.43012822 \backslash \mathrm{A} 27=111.38138115 \backslash \mathrm{A} 28=$ $109.91828511 \backslash \mathrm{A} 29=109.65802699 \backslash \mathrm{A} 30=89.86785275 \backslash \mathrm{D} 1=178.40067204 \backslash \mathrm{D} 2=-66.2$ $9532724 \backslash D 3=55.67399414 \backslash D 4=171.76229967 \backslash D 5=10.15203796 \backslash D 6=102.57985811 \backslash$ $\mathrm{D} 7=-174.95979184 \backslash \mathrm{D} 8=-53.78971269 \backslash \mathrm{D} 9=67.95881995 \backslash \mathrm{D} 10=-61.30028854 \backslash \mathrm{D} 11=-$ $119.3555044 \backslash D 12=120.32545641 \backslash D 13=60.92871766 \backslash D 14=-122.35888935 \backslash D 15=118$ $.67387775 \backslash \mathrm{D} 16=106.21187794 \backslash \mathrm{D} 17=-179.76621529 \backslash \mathrm{D} 18=0.02519765 \backslash \mathrm{D} 19=0.0161$ $3592 \backslash \mathrm{D} 20=0.04305114 \backslash \mathrm{D} 21=-180.02200919 \backslash \mathrm{D} 22=-179.79744188 \backslash \mathrm{D} 23=180.146764$ $69 \backslash D 24=-179.85770283 \backslash D 25=180.0763185 \backslash D 26=-58.02109584 \backslash D 27=-120.1186253$ $1 \backslash \mathrm{D} 28=120.5100964 \backslash \mathrm{D} 29=-175.97427552 \backslash \backslash$ Version=DEC-AXP-OSF $/ 1-G 98 \mathrm{RevA} .11$. $3 \backslash \mathrm{HF}=-1356.0904085 \backslash \mathrm{S} 2=0.756062 \backslash \mathrm{S} 2-1=0 . \backslash \mathrm{S} 2 \mathrm{~A}=0.750028 \backslash \mathrm{RMSD}=8.122 \mathrm{e}-09 \backslash \mathrm{RMS}$ $\mathrm{F}=2.193 \mathrm{e}-05 \backslash \mathrm{Dipole}=1.6064184,0.0385489,0.4576216 \backslash \mathrm{PG}=\mathrm{C} 01 \quad[\mathrm{X}(\mathrm{C} 13 \mathrm{H} 16 \mathrm{~N} 1 \mathrm{~S} 2)$ ]$\backslash \backslash @$

\section{$\mathrm{CH}_{3} \mathrm{SCH}\left(\mathrm{CH}_{2} \mathrm{Ph}\right) \mathrm{S}-\mathrm{C}\left(\mathrm{CH}_{3}\right)_{2} \mathrm{CN}$}

$1 \backslash 1 \backslash G I N C-S C 80 \backslash F O p t \backslash R B 3 L Y P \backslash 6-31 G(d) \backslash C 13 H 17 N 1 S 2 \backslash M L C 501 \backslash 17-J a n-2003 \backslash 0 \backslash \backslash \# N$

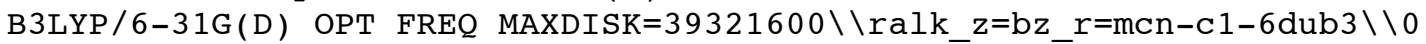
$, 1 \backslash \mathrm{C},-0.6947236938,1.024208585,-0.1454217303 \backslash \mathrm{S}, \overline{1} .1550739378,0.88435048$ $66,-0.0204696639 \backslash \mathrm{S},-1.5360480469,2.2028103869,0.9967636392 \backslash \mathrm{C},-1.480144$ $4822,-0.3094760161,-0.138212312 \backslash \mathrm{C},-1.1126455589,-1.2443003215,-1.27323$ $62416 \backslash \mathrm{H},-1.3578059813,-0.8106217899,0.824154676 \backslash \mathrm{H},-2.5412095863,-0.040$ $3538335,-0.2222891044 \backslash \mathrm{C},-0.7550465872,3.7758458919,0.5004209075 \backslash \mathrm{C}, 1.67$ $89702598,0.3687633707,1.7073077416 \backslash \mathrm{C}, 3.1699296318,-0.0069572895,1.5336$ $081247 \backslash \mathrm{C}, 1.5295595375,1.4964308163,2.7478712833 \backslash \mathrm{C}, 0.9485658241,-0.8236$ $015641,2.1559207251 \backslash \mathrm{H}, 2.0953501407,2.3709492625,2.4127893821 \backslash \mathrm{H}, 1.93496$ $39685,1.1650479973,3.7114306834 \backslash \mathrm{H}, 0.4848247511,1.7796230967,2.88324558$ 
$51 \backslash \mathrm{H},-0.9839236688,4.0186752263,-0.5416939591 \backslash \mathrm{H}, 0.3281299608,3.7362968$ $718,0.6400952887 \backslash \mathrm{H},-1.1755552317,4.551861814,1.1454647232 \backslash \mathrm{C},-0.4027674$ $997,-2.4281375979,-1.0379501223 \backslash C,-0.0777954395,-3.2847508846,-2.09167$ $02784 \backslash \mathrm{C},-0.4568923724,-2.9699399397,-3.3969903766 \backslash \mathrm{C},-1.1703415268,-1.7$ $947504637,-3.6428850143 \backslash \mathrm{C},-1.4970072885,-0.9434239465,-2.5878530763 \backslash \mathrm{H}$, $-2.0682092659,-0.0381738619,-2.7872772409 \backslash \mathrm{H},-1.4797585734,-1.545524395$ $5,-4.6546949789 \backslash \mathrm{H},-0.2043767992,-3.6373427262,-4.2167433479 \backslash \mathrm{H}, 0.471384$ $3139,-4.2007941124,-1.8896583634 \backslash \mathrm{H},-0.1079049101,-2.6805363778,-0.0231$ $747631 \backslash \mathrm{H}, 3.2943463593,-0.8352610875,0.831927659 \backslash \mathrm{H}, 3.5888164116,-0.2976$ $724114,2.5027077357 \backslash \mathrm{H}, 3.7260739027,0.859147578,1.1611222624 \backslash \mathrm{N}, 0.396219$ $6144,-1.772038775,2.538332807 \backslash \mathrm{H},-0.7610621718,1.4549123556,-1.15189727$ $32 \backslash \backslash$ Version=DEC-AXP-OSF / 1-G98RevA. $11.3 \backslash \mathrm{HF}=-1356.7324312 \backslash \mathrm{RMSD}=4.442 \mathrm{e}-09$ $\backslash \mathrm{RMSF}=3.440 \mathrm{e}-06 \backslash \mathrm{Dipole}=0.7274564,1.1981737,-0.4047413 \backslash \mathrm{PG}=\mathrm{C} 01 \quad[\mathrm{X}(\mathrm{C} 13 \mathrm{H} 17$ $\mathrm{N} 1 \mathrm{~S} 2$ ) $] \backslash \backslash \mathrm{Q}$

\section{$\mathrm{CH}_{3} \mathrm{SC} \cdot(\mathrm{Ph}) \mathrm{S}-\mathrm{C}\left(\mathrm{CH}_{3}\right)_{2} \mathrm{CN}(36)$}

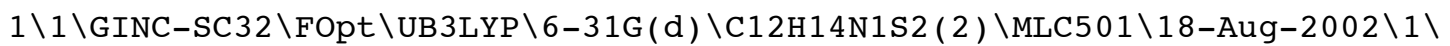
$\backslash \# N$ UB3LYP/6-31G(D) FREQ MAXDISK=131072000 FOPT $=(\mathrm{Z}-\mathrm{MATRIX}, \mathrm{MAXCYC}=100)$ $\mathrm{SCF}=(\mathrm{QC}, \mathrm{TIGHT}, \mathrm{MAXCYC}=100) \backslash \backslash \mathrm{RRAD}(\mathrm{Z}=\mathrm{Ph} \mathrm{R}=\mathrm{MCN}) \mathrm{C} 1 \mathrm{ax} / / \mathrm{B} 3 \backslash \backslash 0,2 \backslash \mathrm{C} \backslash \mathrm{S}, 1, \mathrm{~B} 1 \backslash \mathrm{S}, 1$ , $\mathrm{B} 2,2, \mathrm{~A} 1 \backslash \mathrm{C}, 1, \mathrm{~B} 3,2, \mathrm{~A} 2,3, \mathrm{D} 1,0 \backslash \mathrm{C}, 3, \mathrm{~B} 4,1, \mathrm{~A} 3,2, \mathrm{D} 2,0 \backslash \mathrm{C}, 2, \mathrm{~B} 5,1, \mathrm{~A} 4,3, \mathrm{D} 3,0 \backslash \mathrm{C}, 6$, $\mathrm{B} 6,2, \mathrm{~A} 5,1, \mathrm{D} 4,0 \backslash \mathrm{C}, 6, \mathrm{~B} 7,2, \mathrm{~A} 6,1, \mathrm{D} 5,0 \backslash \mathrm{C}, 6, \mathrm{~B} 8,2, \mathrm{~A} 7,1, \mathrm{D} 6,0 \backslash \mathrm{H}, 8, \mathrm{~B} 9,6, \mathrm{~A} 8,2$, $\mathrm{D} 7$, $0 \backslash \mathrm{H}, 8, \mathrm{~B} 10,6, \mathrm{~A} 9,10, \mathrm{D} 8,0 \backslash \mathrm{H}, 8, \mathrm{~B} 11,6, \mathrm{~A} 10,10, \mathrm{D} 9,0 \backslash \mathrm{H}, 5, \mathrm{~B} 12,3, \mathrm{~A} 11,1, \mathrm{D} 10,0 \backslash \mathrm{H}, 5$ , $\mathrm{B} 13,3, \mathrm{~A} 12,13, \mathrm{D} 11,0 \backslash \mathrm{H}, 5, \mathrm{~B} 14,3, \mathrm{~A} 13,13, \mathrm{D} 12,0 \backslash \mathrm{C}, 4, \mathrm{~B} 15,1, \mathrm{~A} 14,2, \mathrm{D} 13,0 \backslash \mathrm{C}, 4, \mathrm{~B}$ $16,1, \mathrm{~A} 15,16, \mathrm{D} 14,0 \backslash \mathrm{C}, 16, \mathrm{~B} 17,4, \mathrm{~A} 16,1, \mathrm{D} 15,0 \backslash \mathrm{C}, 17, \mathrm{~B} 18,4, \mathrm{~A} 17,1, \mathrm{D} 16,0 \backslash \mathrm{C}, 18, \mathrm{~B}$ $19,16, \mathrm{~A} 18,4, \mathrm{D} 17,0 \backslash \mathrm{H}, 16, \mathrm{~B} 20,4, \mathrm{~A} 19,18, \mathrm{D} 18,0 \backslash \mathrm{H}, 17, \mathrm{~B} 21,4, \mathrm{~A} 20,19, \mathrm{D} 19,0 \backslash \mathrm{H}, 18$ , $\mathrm{B} 22,16, \mathrm{~A} 21,20, \mathrm{D} 20,0 \backslash \mathrm{H}, 19, \mathrm{~B} 23,17, \mathrm{~A} 22,4, \mathrm{D} 21,0 \backslash \mathrm{H}, 20, \mathrm{~B} 24,18, \mathrm{~A} 23,16, \mathrm{D} 22,0 \backslash$ $\mathrm{H}, 9, \mathrm{~B} 25,6, \mathrm{~A} 24,2, \mathrm{D} 23,0 \backslash \mathrm{H}, 9, \mathrm{~B} 26,6, \mathrm{~A} 25,26, \mathrm{D} 24,0 \backslash \mathrm{H}, 9, \mathrm{~B} 27,6, \mathrm{~A} 26,26, \mathrm{D} 25,0 \backslash \mathrm{X}$, $7,1 ., 6,90 ., 2, \mathrm{D} 26,0 \backslash \mathrm{N}, 7, \mathrm{~B} 28,29, \mathrm{~A} 27,6,180.0 \backslash \backslash \mathrm{D} 2=-35.09021444 \backslash \mathrm{D} 3=-70.940$ $85775 \backslash D 6=197.13342316 \backslash D 5=-41.88397446 \backslash D 4=80.53324022 \backslash B 1=1.76266592 \backslash B 2=$ $1.76491418 \backslash \mathrm{B} 3=1.45038859 \backslash \mathrm{B} 4=1.83068375 \backslash \mathrm{B} 5=1.90935687 \backslash \mathrm{B} 6=1.46759559 \backslash \mathrm{B} 7=$ $1.53462562 \backslash B 8=1.54173988 \backslash B 9=1.09360837 \backslash B 10=1.09238336 \backslash B 11=1.09614805 \backslash B$ $12=1.08974979 \backslash \mathrm{B} 13=1.09349872 \backslash \mathrm{B} 14=1.09169781 \backslash \mathrm{B} 15=1.42135308 \backslash \mathrm{B} 16=1.42224$ $621 \backslash \mathrm{B} 17=1.38833895 \backslash \mathrm{B} 18=1.38875137 \backslash \mathrm{B} 19=1.39890536 \backslash \mathrm{B} 20=1.08377272 \backslash \mathrm{B} 21=1$. $0841309 \backslash \mathrm{B} 22=1.08699759 \backslash \mathrm{B} 23=1.08689321 \backslash \mathrm{B} 24=1.08627917 \backslash \mathrm{B} 25=1.09387261 \backslash \mathrm{B} 2$ $6=1.09408458 \backslash \mathrm{B} 27=1.09534711 \backslash \mathrm{B} 28=1.16249426 \backslash \mathrm{A} 1=120.64780033 \backslash \mathrm{A} 2=120.8256$ $1796 \backslash A 3=104.97948192 \backslash A 4=106.61983892 \backslash A 5=109.1352656 \backslash A 6=111.64536538 \backslash A 7$ $=104.37277628 \backslash A 8=109.71362489 \backslash A 9=111.42341574 \backslash A 10=109.70032951 \backslash A 11=111$ $.61813326 \backslash A 12=104.93397596 \backslash A 13=110.39152846 \backslash A 14=121.78396563 \backslash A 15=121.3$ $6258547 \backslash \mathrm{A} 16=121.33987705 \backslash \mathrm{A} 17=121.30614498 \backslash \mathrm{A} 18=120.72823875 \backslash \mathrm{A} 19=119.062$ $07754 \backslash \mathrm{A} 20=119.30540768 \backslash \mathrm{A} 21=119.28627841 \backslash \mathrm{A} 22=119.24848809 \backslash \mathrm{A} 23=120.47331$ $105 \backslash A 24=109.67113877 \backslash A 25=111.44932392 \backslash A 26=109.78128488 \backslash A 27=88.66365939$ $\backslash D 1=-181.79082602 \backslash D 7=-59.51250006 \backslash D 8=120.55744976 \backslash D 9=-119.41849859 \backslash D 10$ $=72.18191784 \backslash \mathrm{D} 11=118.21694492 \backslash \mathrm{D} 12=-123.83462359 \backslash \mathrm{D} 13=-13.94994726 \backslash \mathrm{D} 14=1$ $79.91949005 \backslash D 15=180.52028983 \backslash D 16=-180.83850649 \backslash D 17=0.08703076 \backslash D 18=-179$ $.88768344 \backslash \mathrm{D} 19=-181.18633615 \backslash \mathrm{D} 20=-180.19903579 \backslash \mathrm{D} 21=-180.15406071 \backslash \mathrm{D} 22=17$ $9.92619888 \backslash \mathrm{D} 23=64.07024737 \backslash \mathrm{D} 24=-120.59077904 \backslash \mathrm{D} 25=119.51217784 \backslash \mathrm{D} 26=-226$ $.78647794 \backslash \backslash$ Version=DEC-AXP-OSF $/ 1-G 98 R e v A .11 .3 \backslash \mathrm{HF}=-1316.7874212 \backslash \mathrm{S} 2=0.77$ $0033 \backslash \mathrm{S} 2-1=0 . \backslash \mathrm{S} 2 \mathrm{~A}=0.750307 \backslash \mathrm{RMSD}=0.000 \mathrm{e}+00 \backslash \mathrm{RMSF}=6.792 \mathrm{e}-05 \backslash \mathrm{Dipole}=-1.1619$ $908,-0.0511278,0.0690447 \backslash \mathrm{PG}=\mathrm{C} 01 \quad[\mathrm{X}(\mathrm{C} 12 \mathrm{H} 14 \mathrm{~N} 1 \mathrm{~S} 2)] \backslash \backslash @$

\section{$\mathrm{CH}_{3} \mathrm{SCH}(\mathrm{Ph}) \mathrm{S}-\mathrm{C}\left(\mathrm{CH}_{3}\right)_{2} \mathrm{CN}$}

$1 \backslash 1 \backslash G I N C-S C 28 \backslash F O p t \backslash R B 3 L Y P \backslash 6-31 G(d) \backslash C 12 H 15 N 1 S 2 \backslash M L C 501 \backslash 17-J a n-2003 \backslash 0 \backslash \backslash \# N$ B3LYP/6-31G(D) OPT FREQ MAXDISK=39321600\\ralk_z=ph_r=mcn-clax-6dub3 \ $\backslash 0,1 \backslash \mathrm{C},-0.5441914018,-0.1065140254,-0.384070938 \overline{8} \backslash \mathrm{S}, 1.2707687337,-0.175$ $7428556,0.0290697493 \backslash \mathrm{S},-1.3024271835,1.5688733575,-0.3504684855 \backslash \mathrm{C},-1.2$ $200733102,-1.1944896466,0.4559129483 \backslash C,-0.7793097273,2.2567799283,1.26$ $0980233 \backslash \mathrm{C}, 2.1438270523,0.8567172208,-1.2698326017 \backslash \mathrm{C}, 2.1052383765,2.276$ 
$9676443,-0.8831893629 \backslash \mathrm{C}, 1.5620977585,0.6721047097,-2.6812473856 \backslash \mathrm{C}, 3.61$ $3204771,0.3784006583,-1.2165766042 \backslash \mathrm{H}, 1.5651721268,-0.3899602457,-2.945$ $7977174 \backslash \mathrm{H}, 0.5448771897,1.0639065205,-2.7536095788 \backslash \mathrm{H}, 2.181840564,1.2130$ $921575,-3.4051947523 \backslash \mathrm{H}, 0.2976250205,2.4274786795,1.2818213551 \backslash \mathrm{H},-1.293$ $0061018,3.21826863,1.345964941 \backslash \mathrm{H},-1.0778087952,1.6150726973,2.09299257$ $72 \backslash \mathrm{C},-0.7936141709,-2.5256701158,0.3106648647 \backslash \mathrm{C},-2.2780650632,-0.92698$ $18108,1.331312491 \backslash \mathrm{C},-1.4019625636,-3.5539968209,1.0280508706 \backslash \mathrm{C},-2.8852$ $442124,-1.9570182303,2.0528084434 \backslash \mathrm{C},-2.4486714938,-3.2728680329,1.9081$ $583633 \backslash \mathrm{H}, 0.0217009753,-2.7570004717,-0.3687041876 \backslash \mathrm{H},-2.651877704,0.086$ $0362342,1.4274373153 \backslash \mathrm{H},-1.0578050436,-4.57636079,0.8963302061 \backslash \mathrm{H},-3.706$ $1944374,-1.7251290308,2.7260622001 \backslash \mathrm{H},-2.9226322503,-4.0733086759,2.469$ $7172749 \backslash \mathrm{H}, 3.6711197931,-0.6666026815,-1.5336562709 \backslash \mathrm{H}, 4.0243507536,0.46$ $43096697,-0.2064656925 \backslash \mathrm{H}, 4.224509578,0.9899012329,-1.8886176725 \backslash \mathrm{N}, 2.12$ $68499583,3.3975761574,-0.5765186178 \backslash \mathrm{H},-0.6427022699,-0.3834139315,-1.4$ $380978193 \backslash \backslash$ Version=DEC-AXP-OSF / 1-G98RevA. $11.3 \backslash \mathrm{HF}=-1317.4166164 \backslash \mathrm{RMSD}=5$. $328 \mathrm{e}-09 \backslash \mathrm{RMSF}=1.899 \mathrm{e}-06 \backslash \mathrm{Dipole}=-0.0130986,-1.3740767,-0.2936659 \backslash \mathrm{PG}=\mathrm{C} 01$ $[\mathrm{X}(\mathrm{C} 12 \mathrm{H} 15 \mathrm{~N} 1 \mathrm{~S} 2)] \backslash \backslash @$

\section{$\mathrm{CH}_{3} \mathrm{SC} \cdot\left(\mathrm{CH}_{2} \mathrm{Ph}\right) \mathrm{S}-\mathrm{CH}_{2} \mathrm{Ph}(37)$}

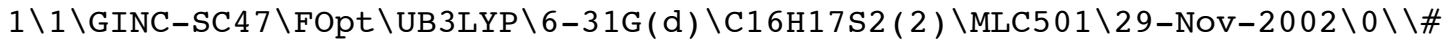
$\mathrm{N}$ B3LYP/6-31G(D) OPT $=($ TIGHT, MAXCYC=100) SCF $=(\mathrm{QC}, \mathrm{TIGHT}, \mathrm{MAXCYC}=100)$ MAXD ISK=131072000 FREQ GUESS=READ GEOM=CHECK \\RRAD(Z=Bz R=Bz) c1bx1 s2=.77 $\backslash \backslash 0,2 \backslash \mathrm{C},-1.4614717747,1.4086931623,0.6848456579 \backslash \mathrm{S},-2.0993383706,2.6758$ $683047,-0.3570429371 \backslash \mathrm{S}, 0.2426341659,1.1643700406,0.9973054226 \backslash \mathrm{C},-2.487$ $2031109,0.4013895222,1.1683663781 \backslash \mathrm{C},-2.39861676,-0.9493788684,0.470116$ $8438 \backslash \mathrm{H},-3.4938332437,0.8201491417,1.037236164 \backslash \mathrm{H},-2.347500676,0.2545186$ $846,2.2475247709 \backslash \mathrm{C},-0.7422527595,3.8879466355,-0.4945029918 \backslash \mathrm{C}, 0.879550$ $1234,0.2873118183,-0.5573407052 \backslash \mathrm{C}, 2.3166736297,-0.094444675,-0.3563102$ $655 \backslash \mathrm{H}, 0.2436160083,-0.5859298621,-0.7114423114 \backslash \mathrm{H}, 0.755363173,0.9739204$ $757,-1.3990074679 \backslash \mathrm{H},-1.1473140531,4.7220977689,-1.0737257985 \backslash \mathrm{H},-0.4387$ $731907,4.2380497168,0.4941021144 \backslash \mathrm{H}, 0.1205613235,3.4682449182,-1.014720$ $1497 \backslash \mathrm{C}, 3.3509401549,0.7931412544,-0.6859386387 \backslash \mathrm{C}, 4.685113191,0.4402724$ $182,-0.4842761492 \backslash \mathrm{C}, 5.0052918379,-0.8070223139,0.0553286185 \backslash \mathrm{C}, 3.983471$ $9902,-1.6975229568,0.3923525525 \backslash \mathrm{C}, 2.6503787509,-1.3421075003,0.1908451$ $672 \backslash \mathrm{H}, 1.8552622996,-2.0358671613,0.4534328817 \backslash \mathrm{H}, 4.2253900578,-2.671018$ $0389,0.8108179316 \backslash \mathrm{H}, 6.0445514041,-1.0842405334,0.2105355045 \backslash \mathrm{H}, 5.474667$ $0885,1.1381039349,-0.7506093318 \backslash \mathrm{H}, 3.1063314933,1.7653958577,-1.1079215$ $117 \backslash \mathrm{C},-1.8103559569,-2.048548613,1.1079998748 \backslash \mathrm{C},-1.7010227203,-3.27981$ $89345,0.4566411093 \backslash \mathrm{C},-2.1826605875,-3.4288896931,-0.8445460339 \backslash \mathrm{C},-2.77$ $56543255,-2.3400638306,-1.488951393 \backslash \mathrm{C},-2.8808651348,-1.1117347639,-0.8$ $367252567 \backslash \mathrm{H},-3.3397562395,-0.267515043,-1.3466083656 \backslash \mathrm{H},-3.1586150331,-$ $2.4484389855,-2.5004940611 \backslash \mathrm{H},-2.1012114503,-4.3862937257,-1.3522571907$ $\backslash \mathrm{H},-1.244808637,-4.1228848337,0.9693940127 \backslash \mathrm{H},-1.4345623372,-1.93744180$ $77,2.1221144314 \backslash \backslash$ Version=DEC-AXP-OSF / 1-G98RevA. $11.3 \backslash \mathrm{HF}=-1416.2768267 \backslash \mathrm{S}$ $2=0.755837 \backslash \mathrm{S} 2-1=0 . \backslash \mathrm{S} 2 \mathrm{~A}=0.750027 \backslash \mathrm{RMSD}=0.000 \mathrm{e}+00 \backslash \mathrm{RMSF}=3.566 \mathrm{e}-06 \backslash \mathrm{Dipole}=0$ $.2523139,-0.0076248,-0.4348361 \backslash \mathrm{PG}=\mathrm{C} 01 \quad[\mathrm{X}(\mathrm{C} 16 \mathrm{H} 17 \mathrm{~S} 2)] \backslash \backslash @$

\section{$\mathrm{CH}_{3} \mathrm{SCH}\left(\mathrm{CH}_{2} \mathrm{Ph}\right) \mathrm{S}-\mathrm{CH}_{2} \mathrm{Ph}$}

$1 \backslash 1 \backslash G I N C-S C 6 \backslash F O p t \backslash R B 3 L Y P \backslash 6-31 G(d) \backslash C 16 H 18 S 2 \backslash M L C 501 \backslash 16-J a n-2003 \backslash 0 \backslash \backslash \# \mathrm{~N}$ B3

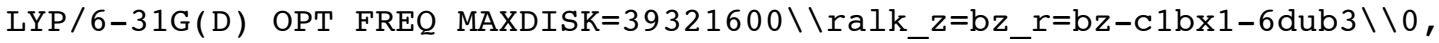
$1 \backslash \mathrm{C}, 1.0567466473,-1.0197920906,-1.7144864491 \backslash \mathrm{S}, 2 . \overline{6} 722049014,-0.7148884$ $057,-0.875421035 \backslash \mathrm{S},-0.1674741192,0.3696329174,-1.6086680918 \backslash \mathrm{C}, 0.502220$ $879,-2.4231217845,-1.3612549719 \backslash \mathrm{C}, 0.060354325,-2.6738679198,0.06587405$ $69 \backslash \mathrm{H}, 1.2784341018,-3.1491803668,-1.6366410854 \backslash \mathrm{H},-0.3467349317,-2.59420$ $00121,-2.0324152937 \backslash \mathrm{C}, 3.3264377882,0.6206405201,-1.9352844165 \backslash \mathrm{C},-0.238$ $9815932,0.8010138438,0.1944071322 \backslash \mathrm{C},-0.9173613432,2.138072631,0.353879$ $6716 \backslash \mathrm{H},-0.7803326052,0.0199707675,0.7305894575 \backslash \mathrm{H}, 0.7908361299,0.826175$ 
$7774,0.5618248979 \backslash \mathrm{H}, 4.2702737036,0.9447594958,-1.4891168169 \backslash \mathrm{H}, 3.517204$ $8845,0.2632448537,-2.9518912745 \backslash \mathrm{H}, 2.6304350326,1.4630142018,-1.9648739$ $887 \backslash \mathrm{C},-0.1815355104,3.3298172462,0.2975021885 \backslash \mathrm{C},-0.811463254,4.5648162$ $138,0.4463231478 \backslash \mathrm{C},-2.1920415643,4.6275517931,0.6484279134 \backslash \mathrm{C},-2.936089$ $4917,3.4479592135,0.7021968952 \backslash \mathrm{C},-2.3019156904,2.2136485381,0.55546190$ $27 \backslash \mathrm{H},-2.8841642279,1.2962544753,0.6010959349 \backslash \mathrm{H},-4.0105931008,3.4875352$ $359,0.8611307342 \backslash \mathrm{H},-2.6837117157,5.5896423529,0.765578409 \backslash \mathrm{H},-0.2245492$ $746,5.4788080961,0.4066271829 \backslash \mathrm{H}, 0.89349596,3.2853636449,0.1390756972 \backslash \mathrm{C}$ $,-1.2971375356,-2.6088168638,0.4109977862 \backslash \mathrm{C},-1.716860842,-2.8420379079$ $, 1.7218870559 \backslash \mathrm{C},-0.7820390578,-3.1521751236,2.7112978486 \backslash \mathrm{C}, 0.570983624$ $7,-3.2347771185,2.3779732721 \backslash \mathrm{C}, 0.9864902512,-3.0030047777,1.0661115402$ $\backslash \mathrm{H}, 2.040807831,-3.0843029338,0.8143152304 \backslash \mathrm{H}, 1.3052260775,-3.4867534328$ , 3.1386842448\H, $-1.1063575097,-3.3371044271,3.7318862607 \backslash \mathrm{H},-2.77433441$ $61,-2.788496961,1.967842017 \backslash \mathrm{H},-2.0293948182,-2.3705874231,-0.356873342$ $1 \backslash \mathrm{H}, 1.2709205667,-1.035614011,-2.7892996791 \backslash \backslash$ Version=DEC-AXP-OSF $/ 1-\mathrm{G} 98$ RevA. $11.3 \backslash \mathrm{HF}=-1416.9220068 \backslash \mathrm{RMSD}=7.576 \mathrm{e}-09 \backslash \mathrm{RMSF}=2.107 \mathrm{e}-06 \backslash \mathrm{Dipole}=0.1222$ $289,-0.0156973,0.0393123 \backslash \mathrm{PG}=\mathrm{C} 01[\mathrm{X}(\mathrm{C} 16 \mathrm{H} 18 \mathrm{~S} 2)] \backslash \backslash @$

\section{$\mathrm{CH}_{3} \mathrm{SC} \cdot(\mathrm{Ph}) \mathrm{S}-\mathrm{CH}_{2} \mathrm{Ph}(38)$}

$1 \backslash 1 \backslash G I N C-S C 97 \backslash F O p t \backslash U B 3 L Y P \backslash 6-31 G(d) \backslash C 15 H 15 S 2$ ( 2$) \backslash M L C 501 \backslash 28-N o v-2002 \backslash 0 \backslash \backslash \#$ $\mathrm{N}$ B3LYP/6-31G(D) OPT $=($ TIGHT, MAXCYC $=100) \quad \mathrm{SCF}=(\mathrm{QC}, \mathrm{TIGHT}, \mathrm{MAXCYC}=100)$ MAXD ISK $=131072000$ FREQ $\backslash \backslash R R A D(Z=P h \quad R=B z) \quad c 1 c x \quad s 2=1.39 \backslash \backslash 0,2 \backslash C,-0.0277767217$, $-1.2203997432,0.5999659775 \backslash S,-0.0489339369,-1.1725510745,2.3706549281 \backslash$ $\mathrm{S}, 1.5174615623,-1.2123976173,-0.2517610955 \backslash \mathrm{C},-1.2757135254,-1.23635292$ $82,-0.1279986748 \backslash \mathrm{C}, 1.1932292566,-2.4302587846,2.8608967633 \backslash \mathrm{C}, 2.3656778$ $049,0.3407983455,0.3592944891 \backslash \mathrm{C}, 1.7974110213,1.6132335008,-0.203441073$ $4 \backslash \mathrm{H}, 2.3253234754,0.3299147564,1.4513370916 \backslash \mathrm{H}, 3.4053249361,0.1913857073$ $, 0.0509755449 \backslash \mathrm{C}, 0.8500636,2.3505487406,0.5197155057 \backslash \mathrm{C}, 2.193142809,2.07$ $918905,-1.4657520982 \backslash \mathrm{C}, 0.3098184235,3.5246527245,-0.0071990753 \backslash \mathrm{C}, 1.655$ $899676,3.2523590906,-1.9926199251 \backslash \mathrm{C}, 0.7105213426,3.978974466,-1.264266$ $8502 \backslash \mathrm{H}, 0.5379640569,2.0012333502,1.5006094463 \backslash \mathrm{H}, 2.9270105505,1.5157890$ $807,-2.0381346404 \backslash \mathrm{H},-0.4232432988,4.085232446,0.5668888337 \backslash \mathrm{H}, 1.9770908$ $217,3.601961753,-2.9703768011 \backslash \mathrm{H}, 0.2918446249,4.8943967924,-1.673737885$ $1 \backslash \mathrm{C},-2.4940634039,-1.631793379,0.4908609075 \backslash \mathrm{C},-1.3392913215,-0.8573744$ $494,-1.4974927996 \backslash \mathrm{C},-3.6891419087,-1.643844879,-0.2156252782 \backslash \mathrm{C},-2.5401$ $242477,-0.8764732527,-2.1932837917 \backslash \mathrm{C},-3.7263902812,-1.2684046796,-1.56$ $28470183 \backslash \mathrm{H},-2.4805249598,-1.9437411163,1.5291937117 \backslash \mathrm{H},-0.43278549,-0.5$ $313131429,-1.9942815471 \backslash \mathrm{H},-4.6008338385,-1.9578281582,0.2863612257 \backslash \mathrm{H},-$ $2.5545015779,-0.573491869,-3.2371422 \backslash \mathrm{H},-4.6637044525,-1.2807923567,-2$. $1120465999 \backslash \mathrm{H}, 1.2468675007,-2.3890673441,3.9522192321 \backslash \mathrm{H}, 2.1737451597,-2$ $.2066557695,2.4373239658 \backslash \mathrm{H}, 0.8744053437,-3.4269679938,2.5472669489 \backslash \backslash \mathrm{Ve}$ rsion=DEC $-\mathrm{AXP}-\mathrm{OSF} / 1-\mathrm{G} 98 \mathrm{RevA} .11 .3 \backslash \mathrm{HF}=-1376.9749829 \backslash \mathrm{S} 2=0.771784 \backslash \mathrm{S} 2-1=0 . \backslash$ $\mathrm{S} 2 \mathrm{~A}=0.750355 \backslash \mathrm{RMSD}=0.000 \mathrm{e}+00 \backslash \mathrm{RMSF}=2.549 \mathrm{e}-07 \backslash \mathrm{Dipole}=0.2609814,0.1112424$, $0.1646758 \backslash \mathrm{PG}=\mathrm{C} 01 \quad[\mathrm{X}(\mathrm{C} 15 \mathrm{H} 15 \mathrm{~S} 2)] \backslash \backslash @$

\section{$\mathrm{CH}_{3} \mathrm{SCH}(\mathrm{Ph}) \mathrm{S}-\mathrm{CH}_{2} \mathrm{Ph}$}

$1 \backslash 1 \backslash G I N C-S C 103 \backslash F O p t \backslash R B 3 L Y P \backslash 6-31 G(d) \backslash C 15 H 16 S 2 \backslash M L C 501 \backslash 25-J a n-2003 \backslash 0 \backslash \backslash \# N$ B3LYP/6-31G(D) OPT FREQ MAXDISK=131072000 GEOM=CHECK GUESS=READ \\ralk $z=p h \_r=b z-c 1 c x-6 d u b 3 \backslash \backslash 0,1 \backslash C,-1.6442979441,-0.6136439717,-0.5301616163 \backslash$ $\mathrm{S},-1.9986958778,-1.3629663056,1.1239175322 \backslash \mathrm{S},-0.3571158105,-1.50163944$ $85,-1.5372587661 \backslash \mathrm{C},-1.4855564016,0.8953745367,-0.3633163513 \backslash \mathrm{C},-2.66188$ $60908,-2.9919225493,0.6277683055 \backslash \mathrm{C}, 0.9659723259,-2.0244410545,-0.34917$ $61742 \backslash \mathrm{C}, 2.0117864491,-1.0038757942,0.0246280153 \backslash \mathrm{H}, 0.469714339,-2.41546$ $14868,0.5429703574 \backslash \mathrm{H}, 1.423476447,-2.8693388062,-0.8753971038 \backslash \mathrm{C}, 2.02966$ $64923,-0.4431200807,1.3079371389 \backslash \mathrm{C}, 2.997920544,-0.6144514677,-0.893557$ $0677 \backslash \mathrm{C}, 3.0072336042,0.4873386265,1.665746192 \backslash \mathrm{C}, 3.9741821188,0.31559351$ $28,-0.5391595854 \backslash \mathrm{C}, 3.9817995547,0.8700283129,0.7437132657 \backslash \mathrm{H}, 1.26829545$ 
$6,-0.7343466269,2.0271161259 \backslash \mathrm{H}, 2.9958215896,-1.0417611787,-1.893954743$ $5 \backslash \mathrm{H}, 3.0050665794,0.9130857902,2.6657295191 \backslash \mathrm{H}, 4.7325678607,0.6043643525$ $,-1.2622957286 \backslash \mathrm{H}, 4.7437570706,1.5934310873,1.0214591274 \backslash \mathrm{C},-2.607143672$ $7,1.6321996334,0.0536107248 \backslash \mathrm{C},-0.2977888868,1.5810772327,-0.6311685437$ $\backslash \mathrm{C},-2.5390812357,3.0154258894,0.2026086653 \backslash \mathrm{C},-0.2279065344,2.968014266$ $6,-0.4770009334 \backslash \mathrm{C},-1.3444416507,3.6902662429,-0.0600604179 \backslash \mathrm{H},-3.537605$ $4054,1.1130717676,0.2683755602 \backslash \mathrm{H}, 0.5726435677,1.035744852,-0.97473585 \backslash$ $\mathrm{H},-3.41979094,3.5661057846,0.5226870196 \backslash \mathrm{H}, 0.7071452656,3.4801489016,-0$ $.6876307152 \backslash \mathrm{H},-1.2883580889,4.7694393377,0.0559535409 \backslash \mathrm{H},-2.9148948882$, $-3.5197348339,1.5513043969 \backslash \mathrm{H},-1.9207575556,-3.5699941005,0.0702921776 \backslash$ $\mathrm{H},-3.5673662992,-2.8801273357,0.0234262071 \backslash \mathrm{H},-2.5394800199,-0.79411545$ $51,-1.1363098537 \backslash \backslash V e r s i o n=D E C-A X P-O S F / 1-G 98 R e v A .11 .3 \backslash \mathrm{HF}=-1377.6050148 \backslash$ $\mathrm{RMSD}=9.298 \mathrm{e}-09 \backslash \mathrm{RMSF}=2.472 \mathrm{e}-06 \backslash \mathrm{Dipole}=0.0032703,-0.1935558,0.1560251 \backslash \mathrm{PG}$ $=\mathrm{C} 01[\mathrm{X}(\mathrm{C} 15 \mathrm{H} 16 \mathrm{~S} 2)] \backslash \backslash \mathrm{Q}$

\section{$\mathrm{CH}_{3} \mathrm{SC} \cdot\left(\mathrm{CH}_{2} \mathrm{Ph}\right) \mathrm{S}-\mathrm{CH}\left(\mathrm{CH}_{3}\right) \mathrm{Ph}(39)$}

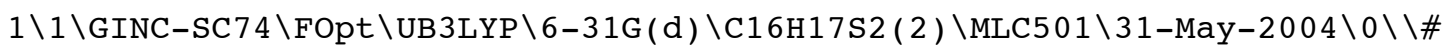
B3LYP/6-31G* OPT FREQ SCF=TIGHT MAXDISK $=402653184$ GEOM=CHECK GUESS=RE $\mathrm{AD} \backslash \backslash \mathrm{rrad} Z \mathrm{Z}=\mathrm{Ph} \_\mathrm{R}=\mathrm{EtPh}-\mathrm{a} 2 \mathrm{~b} 2 \mathrm{c} 1-6 \mathrm{dub} \backslash \backslash \backslash 0,2 \backslash \mathrm{C}, 0.8830576753,0.6463904476,-0$. $88012328 \overline{3} \backslash \mathrm{C}, 2.0197631926,0.1735625795,-0.1161167853 \backslash \mathrm{S}, 0.6453334004,2.3$ $840351898,-1.0572191396 \backslash \mathrm{S},-0.1104387081,-0.4367774874,-1.8574265052 \backslash \mathrm{C}$, $-1.1713014789,2.6111748326,-1.0072197378 \backslash \mathrm{C},-0.8596931158,-1.6092691322$ $,-0.5801588842 \backslash \mathrm{H},-0.0176154187,-1.9534075568,0.0263329163 \backslash \mathrm{C},-1.4288994$ $108,-2.7981754761,-1.361590752 \backslash \mathrm{C},-1.8505077591,-0.9019896008,0.3151611$ $838 \backslash \mathrm{C},-1.4604859548,-0.5097083881,1.6032943826 \backslash \mathrm{C},-3.1549787051,-0.6022$ $623886,-0.1065266085 \backslash \mathrm{C},-2.3459215405,0.162471175,2.4485334216 \backslash \mathrm{C},-4.042$ $0968975,0.0653657946,0.7363267589 \backslash \mathrm{C},-3.6410713503,0.4519233475,2.01813$ $40914 \backslash \mathrm{H},-0.4522660077,-0.7301293168,1.9448972835 \backslash \mathrm{H},-3.4798803777,-0.88$ $45700283,-1.104177831 \backslash \mathrm{H},-2.0223935664,0.4558807317,3.4438185368 \backslash \mathrm{H},-5.0$ $492774532,0.2854063932,0.3917724478 \backslash \mathrm{H},-4.3336879876,0.9718893922,2.674$ $5236039 \backslash \mathrm{H},-0.6365394043,-3.3115553258,-1.9149159973 \backslash \mathrm{H},-1.8883335157,-3$ $.5111104225,-0.6682001508 \backslash \mathrm{H},-2.1940608721,-2.4902397424,-2.0820084076 \backslash$ $\mathrm{H},-1.3385365934,3.6799138804,-1.1677843993 \backslash \mathrm{H},-1.5718559415,2.315278428$ $9,-0.0356476514 \backslash \mathrm{H},-1.6561961178,2.0410028683,-1.8006104713 \backslash \mathrm{C}, 2.6034953$ $252,0.9612004511,0.9126573647 \backslash \mathrm{C}, 2.5929716533,-1.1042375276,-0.35475016$ $53 \backslash \mathrm{C}, 3.6864620942,0.4963247925,1.6483884935 \backslash \mathrm{C}, 3.674515083,-1.559773797$ $6,0.3888766168 \backslash \mathrm{C}, 4.2323602278,-0.7668402473,1.3971458848 \backslash \mathrm{H}, 2.181566242$ $9,1.9356428038,1.1333504836 \backslash \mathrm{H}, 2.1947787236,-1.7155154572,-1.1582331621$ $\backslash \mathrm{H}, 4.1053606999,1.1229320411,2.4317828297 \backslash \mathrm{H}, 4.0957144117,-2.5382552871$ $, 0.1718367311 \backslash \mathrm{H}, 5.0788938704,-1.1262278132,1.9754016637 \backslash \backslash$ Version=DEC $-\mathrm{A}$ $\mathrm{XP}-\mathrm{OSF} / 1-\mathrm{G} 03 \mathrm{RevB} .03 \backslash \mathrm{State}=2-\mathrm{A} \backslash \mathrm{HF}=-1416.2892115 \backslash \mathrm{S} 2=0.768738 \backslash \mathrm{S} 2-1=0 . \backslash \mathrm{S} 2 \mathrm{~A}$ $=0.750266 \backslash \mathrm{RMSD}=6.974 \mathrm{e}-09 \backslash \mathrm{RMSF}=4.782 \mathrm{e}-06 \backslash \mathrm{Dipole}=-0.6484999,-0.3244343,0$ $.5986114 \backslash \mathrm{PG}=\mathrm{C} 01 \quad[\mathrm{X}(\mathrm{C} 16 \mathrm{H} 17 \mathrm{~S} 2)] \backslash \backslash @$

\section{$\mathrm{CH}_{3} \mathrm{SCH}\left(\mathrm{CH}_{2} \mathrm{Ph}\right) \mathrm{S}-\mathrm{CH}\left(\mathrm{CH}_{3}\right) \mathrm{Ph}$}

$1 \backslash 1 \backslash G I N C-S C 160 \backslash F O p t \backslash R B 3 L Y P \backslash 6-31 G(d) \backslash C 16 H 18 S 2 \backslash M L C 501 \backslash 21-J u 1-2004 \backslash 0 \backslash \backslash \# B$ $3 \mathrm{LYP} / 6-31 \mathrm{G} * \mathrm{OPT}=(\mathrm{TIGHT}, \mathrm{MAXCYC}=200)$ FREQ MAXDISK $=402653184 \backslash \backslash \mathrm{RALK} \mathrm{Z}=\mathrm{Ph} \mathrm{R}$ $=\mathrm{EtPh}-\mathrm{a} 2 \mathrm{~b} 2 \mathrm{c} 1-6 \mathrm{dub} 3 \backslash \backslash 0,1 \backslash \mathrm{C}, 0.6096205298,0.7676966945,-0.6988323074 \backslash \mathrm{C}, \overline{1}$. $8472194974,0.2426350277,-0.0027818862 \backslash \mathrm{S}, 1.0880343242,2.177000418,-1.78$ $80593468 \backslash \mathrm{S},-0.2852957211,-0.50505626,-1.7075927585 \backslash \mathrm{C},-0.5461887031,2.9$ $288017063,-2.1174043047 \backslash \mathrm{C},-0.9342321422,-1.6201225945,-0.3669329073 \backslash \mathrm{H}$, $-0.0607948288,-1.9634093622,0.1973247543 \backslash \mathrm{C},-1.5731097891,-2.832924507$, $-1.0624780556 \backslash \mathrm{C},-1.8852437569,-0.9239015176,0.5889732521 \backslash \mathrm{C},-1.60480850$ $59,-0.9025387787,1.9614875922 \backslash \mathrm{C},-3.0690024795,-0.3189264452,0.13941774$ $62 \backslash \mathrm{C},-2.4853633853,-0.3021536759,2.8654332315 \backslash \mathrm{C},-3.9497583645,0.278930$ $2678,1.03873607 \backslash \mathrm{C},-3.6619600022,0.2887451224,2.4066852778 \backslash \mathrm{H},-0.6886478$ $445,-1.3626888976,2.3250388333 \backslash \mathrm{H},-3.2920981401,-0.3072964895,-0.924106$ 
$593 \backslash \mathrm{H},-2.2488546795,-0.2974489586,3.926353447 \backslash \mathrm{H},-4.8629693783,0.740687$ $072,0.6720673177 \backslash \mathrm{H},-4.3489997689,0.7567904012,3.1066041543 \backslash \mathrm{H},-0.845950$ $611,-3.3445023397,-1.7011887751 \backslash \mathrm{H},-1.9348279883,-3.5414204245,-0.30922$ $70346 \backslash \mathrm{H},-2.4262011042,-2.5365334563,-1.681761977 \backslash \mathrm{H},-0.3646490821,3.785$ $8708048,-2.7717903485 \backslash \mathrm{H},-1.0105084694,3.2817133242,-1.1916171229 \backslash \mathrm{H},-1$. $2109074182,2.2237190209,-2.6212904869 \backslash \mathrm{C}, 2.0121190976,0.4602600885,1.37$ $14822358 \backslash \mathrm{C}, 2.852385891,-0.4373249801,-0.7055543392 \backslash \mathrm{C}, 3.1555126286,0.00$ $77788032,2.0324217617 \backslash \mathrm{C}, 3.9972535488,-0.8830874752,-0.0468183023 \backslash \mathrm{C}, 4.1$ $524367627,-0.6637531032,1.3242618511 \backslash \mathrm{H}, 1.2403052994,0.9888142028,1.925$ $8747251 \backslash \mathrm{H}, 2.7260922296,-0.6229225057,-1.7681210439 \backslash \mathrm{H}, 3.2658711755,0.18$ $1702284,3.0996044644 \backslash \mathrm{H}, 4.7692022758,-1.4061384698,-0.6050879691 \backslash \mathrm{H}, 5.04$ $35769186,-1.0166482113,1.83645554 \backslash \mathrm{H},-0.0947411978,1.1479176799,0.04672$ $03037 \backslash \backslash$ Version=DEC-AXP-OSF $/ 1-G 03$ RevB. $03 \backslash$ State $=1-A \backslash H F=-1416.9252355 \backslash$ RMS $\mathrm{D}=2.291 \mathrm{e}-09 \backslash \mathrm{RMSF}=2.625 \mathrm{e}-06 \backslash \mathrm{Dipole}=-0.5194801,-0.1750264,0.7426328 \backslash \mathrm{PG}=\mathrm{C}$ $01[\mathrm{X}(\mathrm{C} 16 \mathrm{H} 18 \mathrm{~S} 2)] \backslash \backslash @$

\section{$\mathrm{CH}_{3} \mathrm{SC} \cdot\left(\mathrm{CH}_{2} \mathrm{Ph}\right) \mathrm{S}-\mathrm{C}\left(\mathrm{CH}_{3}\right)_{2} \mathrm{Ph}(40)$}

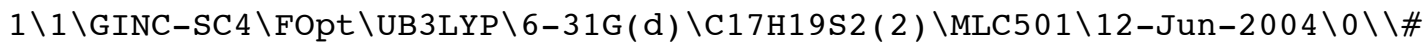
B3LYP $/ 6-31 \mathrm{G} *$ OPT $=($ MAXCYC $=300$, TIGHT $)$ MAXDISK $=402653184$ GEOM=CHECK GUESS $=\mathrm{READ} \backslash \backslash \mathrm{rrad} Z \mathrm{Z}=\mathrm{Ph} \_\mathrm{R}=\mathrm{cumyl}-\mathrm{a} 2 \mathrm{~b} 1 \mathrm{c} 2-6 \mathrm{dub} \backslash \backslash \mathbf{0}, 2 \backslash \mathrm{C}, 1.1096792552,-0.453412587$ $4,-0.3823930143 \backslash \bar{S}, 0.3865179529,-1.9725488564,0.1652537342 \backslash S, 0.12542303$ $29,0.7719642443,-1.1819611342 \backslash \mathrm{C}, 2.5173618997,-0.2243177465,-0.12302573$ $19 \backslash \mathrm{C},-0.7671311164,-2.4374672711,-1.1765669603 \backslash \mathrm{C},-1.0890150874,1.47668$ $80001,0.1344148068 \backslash \mathrm{C},-0.3415660636,1.6475582511,1.459409569 \backslash \mathrm{C},-1.43797$ $63208,2.8679512353,-0.4336595451 \backslash \mathrm{C},-2.3483460252,0.6158529904,0.236389$ $0283 \backslash \mathrm{C},-4.7436304261,-0.8816634401,0.3985213633 \backslash \mathrm{C},-2.7507667584,0.0197$ $508757,1.4396091976 \backslash \mathrm{C},-3.1742773959,0.4354161972,-0.8882944339 \backslash \mathrm{C},-4.35$ $49151551,-0.2998593809,-0.8110065794 \backslash \mathrm{C},-3.9340403639,-0.719997775,1.52$ $04280778 \backslash \mathrm{H},-2.1423655524,0.1246056012,2.3305725892 \backslash \mathrm{H},-2.8825322968,0.8$ $644943417,-1.8426234016 \backslash \mathrm{H},-4.971660561,-0.4199326543,-1.6980431486 \backslash \mathrm{H},-$ $4.2177572495,-1.1700784684,2.4682404887 \backslash \mathrm{H},-5.6642367348,-1.4555242456$, $0.461580435 \backslash \mathrm{C}, 5.2754479146,0.2284375047,0.4049374117 \backslash \mathrm{C}, 3.3909634371,-1$ $.2863028157,0.2417766394 \backslash \mathrm{C}, 3.0925862486,1.072312237,-0.2229182243 \backslash \mathrm{C}, 4$. $4395510464,1.287666942,0.0351434977 \backslash \mathrm{C}, 4.735795131,-1.0580445068,0.5029$ $515368 \backslash \mathrm{H}, 3.0006437745,-2.2960252782,0.2998994982 \backslash \mathrm{H}, 2.459730532,1.90452$ $52895,-0.507977219 \backslash \mathrm{H}, 4.8426222305,2.2938169764,-0.0490850675 \backslash \mathrm{H}, 5.37320$ $02268,-1.8952742602,0.7760933798 \backslash \mathrm{H}, 6.3286412122,0.4016706634,0.6075434$ $126 \backslash \mathrm{H},-1.1915987917,-3.3989123098,-0.8743214351 \backslash \mathrm{H},-0.2271446476,-2.556$ $1780067,-2.1186066338 \backslash \mathrm{H},-1.5667320668,-1.7049826402,-1.2863437011 \backslash \mathrm{H},-0$ $.5476197989,3.5029016985,-0.4750603802 \backslash \mathrm{H},-2.1801978441,3.3476962273,0$. $2155902208 \backslash \mathrm{H},-1.8631617615,2.814522495,-1.4403223424 \backslash \mathrm{H},-0.0070398794,0$ $.6932235798,1.8738009224 \backslash \mathrm{H},-0.9910967821,2.1358710951,2.1965458179 \backslash \mathrm{H}, 0$ $.5389288986,2.2795214295,1.3155351298 \backslash \backslash$ Version=DEC-AXP-OSF / 1-G03RevB . 0 $3 \backslash$ State $=2-A \backslash H F=-1455.5963886 \backslash \mathrm{S} 2=0.770813 \backslash \mathrm{S} 2-1=0 . \backslash \mathrm{S} 2 \mathrm{~A}=0.75033 \backslash \mathrm{RMSD}=4.80$ $3 e-09 \backslash \mathrm{RMSF}=1.997 e-06 \backslash \mathrm{Dipole}=-0.5173462,0.0946596,0.1467993 \backslash \mathrm{PG}=\mathrm{C} 01] \mathrm{X}(\mathrm{C}$ $17 \mathrm{H} 19 \mathrm{~S} 2) \mathrm{]} \backslash \backslash$ @

\section{$\mathrm{CH}_{3} \mathrm{SCH}\left(\mathrm{CH}_{2} \mathrm{Ph}\right) \mathrm{S}-\mathrm{C}\left(\mathrm{CH}_{3}\right)_{2} \mathrm{Ph}$}

$1 \backslash 1 \backslash G I N C-S C 70 \backslash F O p t \backslash R B 3 L Y P \backslash 6-31 G(d) \backslash C 17 H 20 S 2 \backslash M L C 501 \backslash 27-J u 1-2004 \backslash 0 \backslash \backslash \#$ B3 LYP $/ 6-31 \mathrm{G}$ * OPT $=($ TIGHT, $\mathrm{MAXCYC}=200)$ FREQ MAXDISK=402653184 GEOM=CHECK GU $\mathrm{ESS}=\mathrm{READ} \backslash \backslash \mathrm{RALK} Z \mathrm{Z}=\mathrm{Ph} \mathrm{R}=\mathrm{cumy} 1-\mathrm{a} 2 \mathrm{~b} 1 \mathrm{c} 2-6 \mathrm{dub} 3 \backslash \backslash 0,1 \backslash \mathrm{C},-1.0991618768,0.841922$ $7523,-0.887340 \overline{4} 209 \backslash \bar{S},-0.1963612896,2.1390801955,0.0597803832 \backslash S,-0.0787$ $363175,-0.5633292774,-1.5830018046 \backslash \mathrm{C},-2.3784355052,0.4075789802,-0.180$ $7519402 \backslash \mathrm{C}, 0.9631252789,2.7204459001,-1.2195469348 \backslash \mathrm{C}, 0.8226169992,-1.48$ $2340037,-0.2015616432 \backslash \mathrm{C},-0.125752039,-1.8019002131,0.9592914443 \backslash \mathrm{C}, 1.21$ $41502228,-2.8111986022,-0.8917429637 \backslash C, 2.0988520491,-0.7448991228,0.22$ $82498125 \backslash \mathrm{C}, 4.5326596645,0.4813680235,0.9861192187 \backslash \mathrm{C}, 2.3892553851,-0.48$ 
$13990038,1.5733956983 \backslash \mathrm{C}, 3.0606639448,-0.3798093184,-0.7300446216 \backslash \mathrm{C}, 4.2$ $602958071,0.2239037369,-0.3597006147 \backslash C, 3.5905636521,0.1263324027,1.948$ $6412875 \backslash \mathrm{H}, 1.6766919924,-0.742138656,2.3475537078 \backslash \mathrm{H}, 2.8605952241,-0.559$ $465384,-1.782525528 \backslash \mathrm{H}, 4.9838316336,0.494539184,-1.1246711904 \backslash \mathrm{H}, 3.78371$ $16847,0.3220077961,3.0003300631 \backslash \mathrm{H}, 5.4671594103,0.9533150533,1.27763895$ $66 \backslash C,-4.8078482631,-0.4002098223,0.9978359942 \backslash C,-2.6520787407,0.690484$ $7173,1.1626914725 \backslash \mathrm{C},-3.3467820935,-0.2834096926,-0.9257664541 \backslash \mathrm{C},-4.548$ $3493519,-0.6830017772,-0.345510479 \backslash \mathrm{C},-3.855368774,0.2875089257,1.74742$ $38763 \backslash \mathrm{H},-1.9292561606,1.2310158591,1.7661613373 \backslash \mathrm{H},-3.1486067659,-0.517$ $1307183,-1.9685595775 \backslash \mathrm{H},-5.2839069279,-1.2139778228,-0.9440965492 \backslash \mathrm{H},-4$ $.0447902909,0.517919359,2.792502386 \backslash \mathrm{H},-5.7446458901,-0.7107935625,1.45$ $25618535 \backslash \mathrm{H}, 1.5396557574,3.529542188,-0.7633219178 \backslash \mathrm{H}, 0.4267382705,3.112$ $4137341,-2.0894695816 \backslash \mathrm{H}, 1.6419879472,1.9215913369,-1.5241504202 \backslash \mathrm{H}, 0.32$ $16712541,-3.3780542069,-1.1747839933 \backslash \mathrm{H}, 1.8077093874,-3.4138061717,-0.1$ $942544279 \backslash \mathrm{H}, 1.8186271795,-2.6494490671,-1.7893279069 \backslash \mathrm{H},-0.4273531999,-$ $0.9090254838,1.5096243331 \backslash \mathrm{H}, 0.3637521362,-2.4878431878,1.6620709316 \backslash \mathrm{H}$, $-1.0296574423,-2.2880990377,0.5841724744 \backslash \mathrm{H},-1.3927916422,1.3071570033$, $-1.8360086011 \backslash \backslash$ Version=DEC-AXP-OSF /1-G03RevB.03 \State $=1-\mathrm{A} \backslash \mathrm{HF}=-1456.223$ $8747 \backslash \mathrm{RMSD}=3.051 \mathrm{e}-09 \backslash \mathrm{RMSF}=3.044 \mathrm{e}-06 \backslash \mathrm{Dipole}=0.09113,-0.1601463,0.0614648$ $\backslash \mathrm{PG}=\mathrm{C} 01 \quad[\mathrm{X}(\mathrm{C} 17 \mathrm{H} 20 \mathrm{~S} 2)] \backslash \backslash \mathrm{Q}$

\section{$\mathrm{CH}_{3} \mathrm{SC} \cdot\left(\mathrm{CH}_{2} \mathrm{Ph}\right) \mathrm{S}-\mathrm{CH}_{2} \mathrm{COOCH}_{3}(41)$}

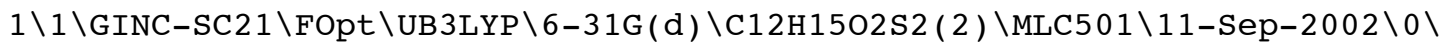
$\backslash \# \mathrm{~N}$ UB3LYP/6-31G(D) $\mathrm{SCF}=(\mathrm{TIGHT}, \mathrm{MAXCYC}=100) \quad \mathrm{OPT}=(\mathrm{MAXCYC}=100) \quad \mathrm{FREQ}$ MAXDI $\mathrm{SK}=131072000 \mathrm{GEOM}=\mathrm{CHECK} \backslash \backslash \mathrm{RRAD}(\mathrm{Z}=\mathrm{BZ} \quad \mathrm{R}=\mathrm{MA}) \quad \mathrm{C} 2 \mathrm{D} 2=-135.1 \mathrm{D} 3=35.0 \mathrm{D} 4=100.1$ $\mathrm{D} 5=-139.9 \mathrm{D} 6=-19.0 \mathrm{D} 7=26.6 \mathrm{D} 8=-94.8 \mathrm{D} 9=146.7 \backslash \backslash 0,2 \backslash \mathrm{C}, 0.0106144613,0.63$ $94493356,0.055012908 \backslash \mathrm{S},-0.2081425176,2.3127865003,0.5580776206 \backslash \mathrm{S},-0.52$ $66335935,-0.6506483405,1.1203801117 \backslash \mathrm{C}, 0.9244422833,0.2578956307,-1.089$ $5701818 \backslash \mathrm{C},-0.9926664675,3.0543514592,-0.9314065251 \backslash \mathrm{C},-2.2996364599,-0$. $2072630242,1.425583443 \backslash \mathrm{C}, 2.2988311279,-0.2284351617,-0.6407496292 \backslash \mathrm{H}, 0$. $439953811,-0.5287777895,-1.6816927868 \backslash \mathrm{H}, 1.0450879835,1.1263495737,-1.7$ $477628181 \backslash \mathrm{C},-3.1310720782,-0.4280002157,0.1840532664 \backslash \mathrm{H},-2.3462696872,0$ $.8422373836,1.7212379072 \backslash \mathrm{H},-2.6264541194,-0.8513593961,2.2441212612 \backslash 0$, $-3.229974273,0.3597209174,-0.7351306789 \backslash 0,-3.7318068449,-1.6355097444$, $0.2022597776 \backslash \mathrm{C},-4.4953000781,-1.9617103961,-0.9713159759 \backslash \mathrm{H},-5.29671370$ $45,-1.2337875422,-1.1225793541 \backslash \mathrm{H},-3.8517315575,-1.971376421,-1.8550414$ $997 \backslash \mathrm{H},-4.906861465,-2.953879961,-0.7850362567 \backslash \mathrm{H},-1.9196058953,2.521199$ $7161,-1.150580256 \backslash \mathrm{H},-0.3207783111,3.0275588036,-1.7934637035 \backslash \mathrm{H},-1.2040$ $171826,4.0973387912,-0.6793988724 \backslash \mathrm{C}, 3.2655140009,0.6817168387,-0.18957$ $4798 \backslash \mathrm{C}, 2.6141358686,-1.5923566945,-0.6527862684 \backslash \mathrm{C}, 4.5160813081,0.23732$ $60476,0.2372819287 \backslash C, 3.8659935778,-2.0400023896,-0.224618027 \backslash C, 4.82074$ $94052,-1.1262641294,0.2215341959 \backslash \mathrm{H}, 3.0318540912,1.7434462254,-0.170465$ $3644 \backslash \mathrm{H}, 1.8744670868,-2.3087587396,-1.002646815 \backslash \mathrm{H}, 5.255342006,0.9564170$ $128,0.5808775484 \backslash \mathrm{H}, 4.0939854045,-3.1026542031,-0.243511675 \backslash \mathrm{H}, 5.7962865$ $649,-1.4720971974,0.5529201588 \backslash \backslash$ Version=DEC-AXP-OSF / 1-G98RevA. $11.3 \backslash \mathrm{HF}=$ $-1413.0968388 \backslash \mathrm{S} 2=0.756077 \backslash \mathrm{S} 2-1=0 . \backslash \mathrm{S} 2 \mathrm{~A}=0.750027 \backslash \mathrm{RMSD}=6.211 \mathrm{e}-09 \backslash \mathrm{RMSF}=2.0$ $82 e-06 \backslash \mathrm{Dipole}=-0.5690163,-0.2843149,-0.4210236 \backslash \mathrm{PG}=\mathrm{C} 01[\mathrm{X}(\mathrm{C} 12 \mathrm{H} 1502 \mathrm{~S} 2)] \backslash$ $1 \mathrm{a}$

\section{$\mathrm{CH}_{3} \mathrm{SCH}\left(\mathrm{CH}_{2} \mathrm{Ph}\right) \mathrm{S}-\mathrm{CH}_{2} \mathrm{COOCH}_{3}$}

$1 \backslash 1 \backslash G I N C-S C 95 \backslash F O p t \backslash R B 3 L Y P \backslash 6-31 G(d) \backslash C 12 H 1602 S 2 \backslash M L C 501 \backslash 16-J a n-2003 \backslash 0 \backslash \backslash \# N$

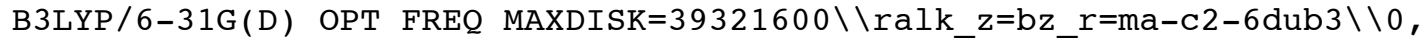
$1 \backslash \mathrm{C}, 0.3877861482,-0.4839259577,-0.3200136192 \backslash \mathrm{S}, \overline{2} .141 \overline{8} 560637,-0.2623923$ $96,0.2344072554 \backslash S,-0.5923456346,1.0695511162,-0.1257722223 \backslash \mathrm{C},-0.355739$ $7242,-1.6558744355,0.3646318742 \backslash \mathrm{C}, 3.0040446397,-1.0491937519,-1.179221$ $2812 \backslash \mathrm{C}, 0.2697436986,2.1432095704,-1.3444490525 \backslash \mathrm{C},-0.6670576171,-1.5052$ $555631,1.8404409288 \backslash \mathrm{H},-1.2916974302,-1.8093471222,-0.1877555076 \backslash \mathrm{H}, 0.25$ 
$66509469,-2.5513472029,0.2039593357 \backslash \mathrm{C}, 0.0267036966,1.6818053896,-2.762$ $488791 \backslash \mathrm{H}, 1.3411568852,2.1167857877,-1.1278835852 \backslash \mathrm{H},-0.1226455668,3.149$ $5583176,-1.186464195 \backslash 0,0.6239345919,0.7699753384,-3.304457191 \backslash 0,-0.959$ $4174872,2.3808285855,-3.3545921975 \backslash \mathrm{C},-1.3027907174,1.9614100923,-4.686$ $8024413 \backslash \mathrm{H},-0.4377270901,2.0435824625,-5.3498644008 \backslash \mathrm{H},-1.6515635802,0.9$ $253795917,-4.6819797545 \backslash \mathrm{H},-2.099181395,2.633205808,-5.007266765 \backslash \mathrm{H}, 2.69$ $91658951,-0.5801845643,-2.1184323036 \backslash \mathrm{H}, 2.8174764129,-2.1264190804,-1.2$ $19255081 \backslash \mathrm{H}, 4.0739615247,-0.8857585043,-1.0229064564 \backslash \mathrm{C}, 0.2797243027,-1$. $8380321162,2.8199199165 \backslash \mathrm{C},-1.9233820804,-1.0432786396,2.2569366335 \backslash \mathrm{C},-$ $0.0170000787,-1.6985776886,4.1759576311 \backslash \mathrm{C},-2.2229655141,-0.902312757,3$ $.6127451803 \backslash \mathrm{C},-1.2689255226,-1.2285866622,4.577205603 \backslash \mathrm{H}, 1.2550940394,-$ $2.2052910103,2.514799691 \backslash \mathrm{H},-2.6754259539,-0.7957500687,1.5112964781 \backslash \mathrm{H}$, $0.7300232448,-1.9624717522,4.9201614402 \backslash \mathrm{H},-3.2037561571,-0.5433999891$, $3.9137770865 \backslash \mathrm{H},-1.5011660405,-1.1239797554,5.6337828872 \backslash \mathrm{H}, 0.4404831759$ $,-0.6938587169,-1.390909786 \backslash \backslash$ Version=DEC-AXP-OSF $/ 1-G 98$ RevA $.11 .3 \backslash \mathrm{HF}=-14$ $13.7440968 \backslash \mathrm{RMSD}=9.412 \mathrm{e}-09 \backslash \mathrm{RMSF}=6.841 \mathrm{e}-06 \backslash \mathrm{Dipole}=-0.214873,-0.0042356,-$ $0.8653603 \backslash \mathrm{PG}=\mathrm{C} 01 \quad[\mathrm{X}(\mathrm{C} 12 \mathrm{H} 1602 \mathrm{~S} 2)] \backslash \backslash @$

\section{$\mathrm{CH}_{3} \mathrm{SC} \cdot(\mathrm{Ph}) \mathrm{S}-\mathrm{CH}_{2} \mathrm{COOCH}_{3}(42)$}

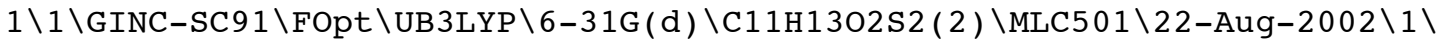
$\backslash \# N$ UB3LYP/6-31G(D) FREQ MAXDISK=131072000 FOPT $=($ Z-MATRIX, MAXCYC $=100)$ $\mathrm{SCF}=(\mathrm{QC}, \mathrm{TIGHT}, \mathrm{MAXCYC}=100) \mathrm{GEOM}=\mathrm{CHECK}$ GUESS=READ $\backslash \backslash \operatorname{RRAD}(\mathrm{Z}=\mathrm{Ph} \mathrm{R}=\mathrm{MA}) \mathrm{c} 1 \mathrm{ay} /$

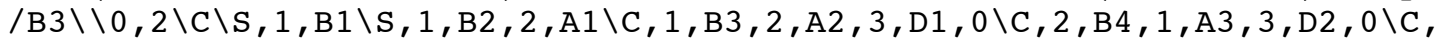
$3, \mathrm{~B} 5,1, \mathrm{~A} 4,2, \mathrm{D} 3,0 \backslash \mathrm{C}, 6, \mathrm{~B} 6,3, \mathrm{~A} 5,1, \mathrm{D} 4,0 \backslash \mathrm{H}, 6, \mathrm{~B} 7,3, \mathrm{~A} 6,1, \mathrm{D} 5,0 \backslash \mathrm{H}, 6, \mathrm{~B} 8,3, \mathrm{~A} 7,1, \mathrm{D}$ $6,0 \backslash 0,7, \mathrm{~B} 9,6, \mathrm{~A} 8,3, \mathrm{D} 7,0 \backslash 0,7, \mathrm{~B} 10,6, \mathrm{~A} 9,10, \mathrm{D} 8,0 \backslash \mathrm{C}, 11, \mathrm{~B} 11,7, \mathrm{~A} 10,6, \mathrm{D} 9,0 \backslash \mathrm{H}, 12$ , $\mathrm{B} 12,11, \mathrm{~A} 11,7, \mathrm{D} 10,0 \backslash \mathrm{H}, 12, \mathrm{~B} 13,11, \mathrm{~A} 12,13, \mathrm{D} 11,0 \backslash \mathrm{H}, 12, \mathrm{~B} 14,11, \mathrm{~A} 13,13, \mathrm{D} 12,0 \backslash$ $\mathrm{H}, 5, \mathrm{~B} 15,2, \mathrm{~A} 14,1, \mathrm{D} 13,0 \backslash \mathrm{H}, 5, \mathrm{~B} 16,2, \mathrm{~A} 15,16, \mathrm{D} 14,0 \backslash \mathrm{H}, 5, \mathrm{~B} 17,2, \mathrm{~A} 16,16, \mathrm{D} 15,0 \backslash \mathrm{C}$, $4, \mathrm{~B} 18,1, \mathrm{~A} 17,2, \mathrm{D} 16,0 \backslash \mathrm{C}, 4, \mathrm{~B} 19,1, \mathrm{~A} 18,19, \mathrm{D} 17,0 \backslash \mathrm{C}, 19, \mathrm{~B} 20,4, \mathrm{~A} 19,1, \mathrm{D} 18,0 \backslash \mathrm{C}, 20$ , B21,4, $\mathrm{A} 20,1, \mathrm{D} 19,0 \backslash \mathrm{C}, 21, \mathrm{~B} 22,19, \mathrm{~A} 21,4, \mathrm{D} 20,0 \backslash \mathrm{H}, 19, \mathrm{~B} 23,4, \mathrm{~A} 22,21, \mathrm{D} 21,0 \backslash \mathrm{H}, 2$ $0, \mathrm{~B} 24,4, \mathrm{~A} 23,22, \mathrm{D} 22,0 \backslash \mathrm{H}, 21, \mathrm{~B} 25,19, \mathrm{~A} 24,23, \mathrm{D} 23,0 \backslash \mathrm{H}, 22, \mathrm{~B} 26,20, \mathrm{~A} 25,4, \mathrm{D} 24,0 \backslash$ $\mathrm{H}, 23, \mathrm{~B} 27,21, \mathrm{~A} 26,19, \mathrm{D} 25,0 \backslash \backslash \mathrm{D} 2=-48.66742221 \backslash \mathrm{D} 3=-59.98700545 \backslash \mathrm{D} 5=159.47267$ $01 \backslash D 4=-80.35076392 \backslash D 6=43.40237843 \backslash D 7=-8.87674609 \backslash B 1=1.76954697 \backslash B 2=1.77$ $231178 \backslash \mathrm{B} 3=1.44369491 \backslash \mathrm{B} 4=1.83547449 \backslash \mathrm{B} 5=1.83134785 \backslash \mathrm{B} 6=1.52496927 \backslash \mathrm{B} 7=1.09$ $587204 \backslash \mathrm{B} 8=1.0936977 \backslash \mathrm{B} 9=1.2088986 \backslash \mathrm{B} 10=1.35071877 \backslash \mathrm{B} 11=1.4390682 \backslash \mathrm{B} 12=1.09$ $317885 \backslash \mathrm{B} 13=1.09020781 \backslash \mathrm{B} 14=1.09294778 \backslash \mathrm{B} 15=1.0933617 \backslash \mathrm{B} 16=1.09122537 \backslash \mathrm{B} 17=$ $1.09255848 \backslash \mathrm{B} 18=1.42267223 \backslash \mathrm{B} 19=1.4224648 \backslash \mathrm{B} 20=1.38817705 \backslash \mathrm{B} 21=1.38816973 \backslash$ $\mathrm{B} 22=1.39949408 \backslash \mathrm{B} 23=1.08353903 \backslash \mathrm{B} 24=1.08443776 \backslash \mathrm{B} 25=1.08696969 \backslash \mathrm{B} 26=1.0870$ $7355 \backslash B 27=1.08653262 \backslash A 1=119.35031129 \backslash A 2=120.11353834 \backslash A 3=104.00387848 \backslash A 4$ $=103.03887394 \backslash A 5=114.56529937 \backslash A 6=106.75033321 \backslash A 7=110.06765083 \backslash A 8=127.0$ $2742896 \backslash A 9=109.08604933 \backslash A 10=115.35654399 \backslash A 11=110.51804799 \backslash A 12=105.5020$ $0456 \backslash A 13=110.43056808 \backslash A 14=106.05484832 \backslash A 15=111.04466808 \backslash A 16=110.586636$ $44 \backslash A 17=121.17625633 \backslash A 18=121.49310815 \backslash A 19=120.9094988 \backslash A 20=121.10236351 \backslash$ $\mathrm{A} 21=120.81033128 \backslash \mathrm{A} 22=118.91779171 \backslash \mathrm{A} 23=119.08284007 \backslash \mathrm{A} 24=119.22802087 \backslash \mathrm{A} 2$ $5=119.36384162 \backslash A 26=120.41384942 \backslash D 1=180.23211003 \backslash D 8=-178.9984738 \backslash D 9=179$ $.00140925 \backslash D 10=-60.56985178 \backslash D 11=-119.64827693 \backslash D 12=120.73334999 \backslash D 13=174$. $05251161 \backslash D 14=-119.06185435 \backslash D 15=118.72690141 \backslash D 16=-201.96568734 \backslash D 17=-180$ $.04187775 \backslash \mathrm{D} 18=-180.56294225 \backslash \mathrm{D} 19=180.5191603 \backslash \mathrm{D} 20=0.13941807 \backslash \mathrm{D} 21=177.506$ $21699 \backslash \mathrm{D} 22=178.84814744 \backslash \mathrm{D} 23=179.23254714 \backslash \mathrm{D} 24=179.36224919 \backslash \mathrm{D} 25=-179.9396$ $5887 \backslash \backslash$ Version=DEC-AXP-OSF $/ 1-G 98$ RevA. $11.3 \backslash \mathrm{HF}=-1373.7927754 \backslash \mathrm{S} 2=0.772138 \backslash$ $\mathrm{S} 2-1=0 . \backslash \mathrm{S} 2 \mathrm{~A}=0.750363 \backslash \mathrm{RMSD}=0.000 \mathrm{e}+00 \backslash \mathrm{RMSF}=8.625 e-05 \backslash \mathrm{Dipole}=0.5647553,0$. $3523014,0.5202045 \backslash \mathrm{PG}=\mathrm{C} 01 \quad[\mathrm{X}(\mathrm{C} 11 \mathrm{H} 1302 \mathrm{~S} 2)] \backslash \backslash @$

\section{$\mathrm{CH}_{3} \mathrm{SCH}(\mathrm{Ph}) \mathrm{S}-\mathrm{CH}_{2} \mathrm{COOCH}_{3}$}

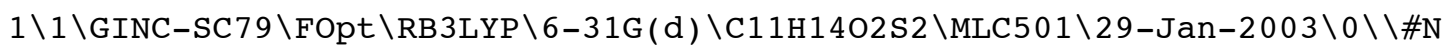
B3LYP/6-31G(D) OPT=TIGHT INT(GRID=ULTRAFINE) FREQ MAXDISK=131072000\\

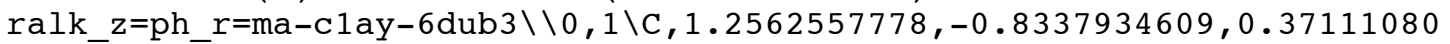
$15 \backslash \mathrm{S}, 1.87 \overline{4} 859121,-1.4854597441,-1.2359285234 \backslash \mathrm{S},-0.3410959786,-1.591207$ 
$8592,0.9899579778 \backslash \mathrm{C}, 1.2865556802,0.6798987907,0.353738545 \backslash \mathrm{C}, 2.10654801$ $52,-3.2491422829,-0.8222843896 \backslash \mathrm{C},-1.4955733138,-1.4896886633,-0.437553$ $9573 \backslash \mathrm{C},-2.3749362362,-0.254441649,-0.4744653666 \backslash \mathrm{H},-2.1319716414,-2.375$ $5210935,-0.3496541494 \backslash \mathrm{H},-0.9224307728,-1.5518040436,-1.3656938879 \backslash 0,-2$ $.3366929961,0.5973222841,-1.3372781588 \backslash 0,-3.2330046141,-0.2335852961,0$ $.563121107 \backslash \mathrm{C},-4.0878784089,0.9197306047,0.6336996762 \backslash \mathrm{H},-3.4908759909,1$ $.8314715784,0.7176603522 \backslash \mathrm{H},-4.6975939432,0.7762906988,1.525959379 \backslash \mathrm{H},-4$ $.7177139926,0.9859905222,-0.2574929385 \backslash \mathrm{H}, 2.4319954035,-3.7439013922,-1$ $.7412803375 \backslash \mathrm{H}, 1.1710585332,-3.6976959163,-0.479362075 \backslash \mathrm{H}, 2.878697415,-3$ $.3770221717,-0.0574695866 \backslash \mathrm{C}, 0.688583427,1.4299488068,-0.6682600732 \backslash \mathrm{C}, 1$ $.9424260087,1.3558098626,1.3920852982 \backslash \mathrm{C}, 0.7438654128,2.8237870039,-0.6$ $445543944 \backslash \mathrm{C}, 1.9969334617,2.7488726125,1.4151240589 \backslash \mathrm{C}, 1.3972231181,3.48$ $82449734,0.3944024992 \backslash \mathrm{H}, 0.1613469721,0.9347452511,-1.4763641995 \backslash \mathrm{H}, 2.41$ $36492579,0.7859292685,2.189807254 \backslash \mathrm{H}, 0.2701871077,3.3889939027,-1.44243$ $55808 \backslash \mathrm{H}, 2.5107016329,3.2547456565,2.2283230756 \backslash \mathrm{H}, 1.4388223569,4.574093$ $4831,0.4089663752 \backslash \mathrm{H}, 1.9414806099,-1.1848895857,1.1495652772 \backslash \backslash$ Version=D EC-AXP-OSF / 1-G98RevA. $11.3 \backslash \mathrm{HF}=-1374.4274538 \backslash \mathrm{RMSD}=4.654 \mathrm{e}-09 \backslash \mathrm{RMSF}=5.312 \mathrm{e}-$ $06 \backslash \mathrm{Dipole}=-0.1765314,-0.2757659,0.4673035 \backslash \mathrm{PG}=\mathrm{C} 01[\mathrm{X}(\mathrm{C} 11 \mathrm{H} 1402 \mathrm{~S} 2)] \backslash \backslash @$

\section{$\mathrm{CH}_{3} \mathrm{SC} \cdot\left(\mathrm{OCH}_{3}\right) \mathrm{SCH}_{2} \mathrm{OCOCH}_{3}(43)$}

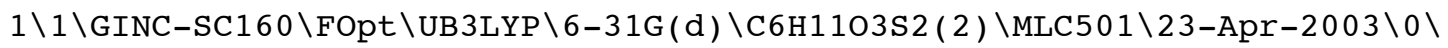
$\backslash \# \mathrm{P}$ B3LYP $/ 6-31 \mathrm{G} * \mathrm{SCF}=(\mathrm{QC}, \mathrm{MAXCYC}=100) \quad \mathrm{OPT}=(\mathrm{MAXCYC}=100) \quad \mathrm{FREQ}$ MAXDISK $=131$ $072000 \backslash \backslash \operatorname{RAFT}(\mathrm{Z}=\mathrm{OMe} \mathrm{R}=\mathrm{VA}) \mathrm{a} 1 \mathrm{~b} 1 \mathrm{c} 1 \mathrm{~d} 3 \mathrm{e} 2 \mathrm{f} 1 / / \mathrm{B} 3 \backslash \backslash 0,2 \backslash \mathrm{S}, 0.3322714222,-1.33810$ $11735,-0.9063561478 \backslash \mathrm{C}, 0.5694748971,-0.5250061298,0.6470647641 \backslash \mathrm{S}, 2.2229$ $45348,-0.384145835,1.2467459401 \backslash \mathrm{C}, 2.3646929606,-1.8402281312,2.3780173$ $258 \backslash \mathrm{C},-1.3096630931,-0.7061140865,-1.4131144165 \backslash 0,-0.4770788066,-0.633$ $9063631,1.515866466 \backslash \mathrm{C},-0.6921128753,0.4861332319,2.3945790952 \backslash 0,-1.229$ $3506832,0.4793060121,-2.2145907541 \backslash \mathrm{C},-1.4569891118,1.6707268399,-1.600$ $2930658 \backslash 0,-1.7749735518,1.7864051526,-0.4377061153 \backslash \mathrm{C},-1.2583630012,2.8$ $07316002,-2.5722486417 \backslash \mathrm{H},-1.7511406959,-1.470831824,-2.0528684105 \backslash \mathrm{H},-1$ $.9148450646,-0.5303100028,-0.5244913106 \backslash \mathrm{H}, 1.5016140583,-1.880452333,3$. $0454963331 \backslash \mathrm{H}, 3.2748778366,-1.6952499913,2.9659377291 \backslash \mathrm{H}, 2.4393739835,-2$ $.7685512536,1.8069091042 \backslash \mathrm{H},-1.8414714853,2.638407595,-3.4826197805 \backslash \mathrm{H},-$ $0.2036999806,2.8589667581,-2.8638987689 \backslash \mathrm{H},-1.5554863394,3.7448716146,-$ $2.1013751664 \backslash \mathrm{H},-1.5256749738,0.1957480346,3.0369865225 \backslash \mathrm{H}, 0.1974766108$, $0.681808446,3.001826327 \backslash \mathrm{H},-0.9555065988,1.3701403213,1.809273606 \backslash \backslash$ Vers ion=DEC-AXP-OSF $/ 1-\mathrm{G} 98 \mathrm{RevA} .11 .3 \backslash \mathrm{HF}=-1257.2539065 \backslash \mathrm{S} 2=0.754973 \backslash \mathrm{S} 2-1=0 . \backslash \mathrm{S} 2$ $\mathrm{A}=0.750018 \backslash \mathrm{RMSD}=0.000 \mathrm{e}+00 \backslash \mathrm{RMSF}=9.849 \mathrm{e}-06 \backslash \mathrm{Dipole}=-0.3783478,0.0568044,0$ $.05398 \backslash \mathrm{PG}=\mathrm{C} 01 \quad \mathrm{X}(\mathrm{C} 6 \mathrm{H} 1103 \mathrm{~S} 2)] \backslash \backslash \mathrm{Q}$

\section{$\mathrm{CH}_{3} \mathrm{SCH}\left(\mathrm{OCH}_{3}\right) \mathrm{SCH}_{2} \mathrm{OCOCH}_{3}$}

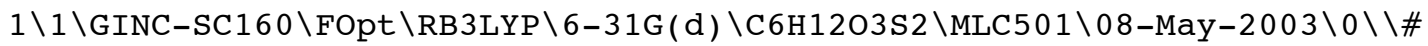
B3LYP $/ 6-31 \mathrm{G} *$ OPT $=($ MAXCYC $=100)$ FREQ MAXDISK $=134217728$ GEOM=CHECK GUESS $=$

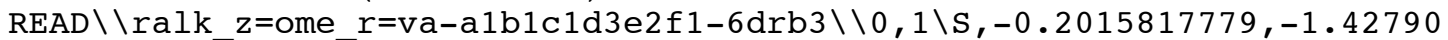
$34543,-0.1 \overline{5} 47220 \overline{2} 72 \backslash \mathrm{C},-0.9383652421,0.2179647681,0.2545016072 \backslash \mathrm{S},-2.540$ $8629392,-0.0458898946,1.1352052248 \backslash \mathrm{C},-3.5602414575,-0.7094865622,-0.23$ $33579927 \backslash \mathrm{C}, 1.238403623,-0.9376664218,-1.161139343 \backslash 0,-1.0536061247,0.96$ $03100383,-0.9216862706 \backslash \mathrm{C},-1.0525619172,2.3746501811,-0.7089736046 \backslash 0,2$. $48742376,-0.8811855308,-0.4470262731 \backslash \mathrm{C}, 2.7632003357,0.2556627682,0.232$ $7923911 \backslash 0,2.0227938086,1.2159304397,0.2779201944 \backslash \mathrm{C}, 4.1021885668,0.1537$ $864236,0.9215943715 \backslash \mathrm{H}, 1.3889552985,-1.7174692039,-1.9075110176 \backslash \mathrm{H}, 1.018$ $7806248,0.0199859847,-1.6337149931 \backslash \mathrm{H},-3.3958247807,-0.1109475676,-1.13$ $16447515 \backslash \mathrm{H},-4.6031053336,-0.6192891984,0.0803542863 \backslash \mathrm{H},-3.3287603693,-1$ $.7570678406,-0.4355418439 \backslash \mathrm{H}, 4.8779373418,-0.140195151,0.2080002027 \backslash \mathrm{H}, 4$ $.0584536804,-0.6210854801,1.6946851078 \backslash \mathrm{H}, 4.3515586415,1.1127033997,1.3$ $768834994 \backslash \mathrm{H},-1.1405520926,2.8287562082,-1.6983807588 \backslash \mathrm{H},-1.905026744,2$. $6798697453,-0.0896681835 \backslash \mathrm{H},-0.1144537416,2.6905540998,-0.2384985344 \backslash \mathrm{H}$, 
$-0.2974820547,0.7249720669,0.9811400417 \backslash \backslash$ Version=DEC-AXP-OSF / 1-G98RevA $.11 .3 \backslash \mathrm{HF}=-1257.9053459 \backslash \mathrm{RMSD}=2.472 \mathrm{e}-09 \backslash \mathrm{RMSF}=4.467 \mathrm{e}-06 \backslash \mathrm{Dipole}=0.5807845$, $0.2671888,-0.5051867 \backslash P G=C 01 \quad[X(C 6 H 1203 S 2)] \backslash \backslash @$

\section{$\mathrm{CH}_{3} \mathrm{SC} \cdot\left(\mathrm{OCH}_{2} \mathrm{CH}_{3}\right) \mathrm{SCH}_{2} \mathrm{OCOCH}_{3}(44)$}

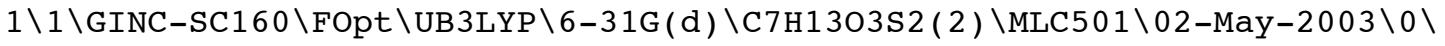
$\backslash$ \#P B3LYP/6-31G(D) OPT $=($ TIGHT, MAXCYC $=100) \quad$ FREQ MAXDISK $=131072000 \backslash \backslash$ RAFT

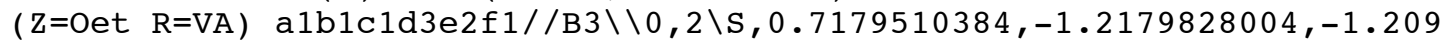
$4187798 \backslash \mathrm{C}, 0.7513503967,-0.5130337722,0.4144629407 \backslash \mathrm{S}, 2.32888654,-0.3148$ $289804,1.1785936357 \backslash \mathrm{C}, 2.4790786921,-1.8499955366,2.1990945022 \backslash \mathrm{C},-0.922$ $1690203,-0.6795000414,-1.8162501935 \backslash 0,-0.3644590199,-0.7420997921,1.16$ $10584614 \backslash C,-0.7268922768,0.282927414,2.1199390976 \backslash 0,-0.8845107286,0.59$ $8510441,-2.4643125658 \backslash \mathrm{C},-1.330927145,1.6738272304,-1.7623576981 \backslash 0,-1.8$ $149024192,1.6109116682,-0.6538458969 \backslash C,-1.1337058677,2.938083929,-2.56$ $17700618 \backslash \mathrm{H},-1.217559603,-1.3987407629,-2.5809555936 \backslash \mathrm{H},-1.6262662646,-0$ $.6667500517,-0.9853062834 \backslash \mathrm{H}, 1.574734655,-1.9962351635,2.7933231884 \backslash \mathrm{H}, 3$ $.3335466449,-1.7030285003,2.8650559757 \backslash \mathrm{H}, 2.6535639048,-2.7215059617,1$. $5638229251 \backslash \mathrm{H},-1.5398818802,2.8212233055,-3.5709082382 \backslash \mathrm{H},-0.0619471979$, $3.1423005533,-2.6617424979 \backslash \mathrm{H},-1.6189995251,3.7708886095,-2.051841517 \backslash \mathrm{C}$ $,-2.076335528,-0.0981906659,2.6978462297 \backslash \mathrm{H},-0.772773959,1.2433691422,1$ $.5993818747 \backslash \mathrm{H}, 0.0455316094,0.335053096,2.8959631061 \backslash \mathrm{H},-2.3747588667,0$. $633544994,3.4566824776 \backslash \mathrm{H},-2.8388838282,-0.1116516092,1.9133015245 \backslash \mathrm{H},-2$ $.0371251082,-1.0867690392,3.1674364716 \backslash \backslash$ Version=DEC-AXP-OSF / 1-G98RevA. $11.3 \backslash \mathrm{HF}=-1296.5733881 \backslash \mathrm{S} 2=0.754954 \backslash \mathrm{S} 2-1=0 . \backslash \mathrm{S} 2 \mathrm{~A}=0.750018 \backslash \mathrm{RMSD}=7.417 \mathrm{e}-09 \backslash$ $\mathrm{RMSF}=1.639 \mathrm{e}-06 \backslash \mathrm{Dipole}=-0.4094067,0.0404173,0.0690797 \backslash \mathrm{PG}=\mathrm{C} 01 \quad[\mathrm{X}(\mathrm{C} 7 \mathrm{H} 1303$ $\mathrm{S} 2 \mathrm{)}] \backslash \backslash @$

\section{$\mathrm{CH}_{3} \mathrm{SCH}\left(\mathrm{OCH}_{2} \mathrm{CH}_{3}\right) \mathrm{SCH}_{2} \mathrm{OCOCH}$}

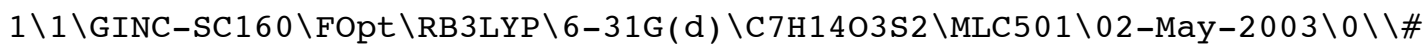
B3LYP $/ 6-31 \mathrm{G} *$ OPT $=($ MAXCYC $=100$, TIGHT $) \quad F R E Q$ MAXDISK $=262144000 \backslash \backslash \mathrm{ralk} z=$ oet $\mathrm{r}=\mathrm{va}-\mathrm{a} 1 \mathrm{~b} 1 \mathrm{c} 1 \mathrm{~d} 3 \mathrm{e} 2 \mathrm{f} 1-6 \mathrm{drb} 3 \backslash \backslash 0,1 \backslash \mathrm{S},-0.0739893389,-1.5513969917,-0.5 \overline{6} 73450$ $872 \backslash \mathrm{C},-0.8560251256,-0.1765366697,0.3943477708 \backslash \mathrm{S},-2.448242767,-0.78691$ $20372,1.1088753887 \backslash \mathrm{C},-3.4479061822,-0.946488676,-0.4169093107 \backslash \mathrm{C}, 1.3378$ $366853,-0.6980940443,-1.3437908034 \backslash 0,-0.9953920625,0.9318822768,-0.439$ $0023206 \backslash \mathrm{C},-1.0282072868,2.1911559806,0.2572235442 \backslash 0,2.5954352199,-0.86$ $16960303,-0.6620913647 \backslash \mathrm{C}, 2.8549573837,-0.026206221,0.3699455278 \backslash 0,2.09$ $52872418,0.8407984706,0.7486423781 \backslash \mathrm{C}, 4.2026441502,-0.3305259484,0.9774$ $111567 \backslash \mathrm{H}, 1.4965050207,-1.1605305737,-2.3178848046 \backslash \mathrm{H}, 1.0877176205,0.357$ $9445256,-1.4472978505 \backslash \mathrm{H},-3.2869157901,-0.0691721631,-1.0467202174 \backslash \mathrm{H},-4$ $.4944804267,-0.9861053543,-0.1052016871 \backslash \mathrm{H},-3.1958922881,-1.8536881595$, $-0.9694709996 \backslash \mathrm{H}, 4.9746848032,-0.3599422351,0.2026481415 \backslash \mathrm{H}, 4.1758374228$ $,-1.318420777,1.4502512711 \backslash \mathrm{H}, 4.4446426168,0.4260862546,1.7244373264 \backslash \mathrm{C}$, $-1.1642377162,3.2901790223,-0.7813435003 \backslash \mathrm{H},-0.1011208719,2.3008837865$, $0.8333454598 \backslash \mathrm{H},-1.8780498109,2.1987197638,0.9525412814 \backslash \mathrm{H},-1.1945964982$ $, 4.2685269831,-0.2893697584 \backslash \mathrm{H},-0.3151686803,3.2785497011,-1.4724902804$ $\backslash \mathrm{H},-2.0842809026,3.166536529,-1.3617885339 \backslash \mathrm{H},-0.2301831649,0.054777783$ $4,1.2608199742 \backslash \backslash$ Version=DEC-AXP-OSF $/ 1-G 98 R e v A .11 .3 \backslash \mathrm{HF}=-1297.224588 \backslash \mathrm{RMS}$ $\mathrm{D}=6.057 \mathrm{e}-09 \backslash \mathrm{RMSF}=1.279 \mathrm{e}-06 \backslash \mathrm{Dipole}=0.5563875,0.4982945,-0.4086041 \backslash \mathrm{PG}=\mathrm{C} 0$ $1[\mathrm{X}(\mathrm{C} 7 \mathrm{H} 14 \mathrm{O} 3 \mathrm{~S} 2)] \backslash \backslash @$

\section{$\mathrm{CH}_{3} \mathrm{SC} \cdot\left(\mathrm{OCH}\left(\mathrm{CH}_{3}\right)_{2}\right) \mathrm{SCH}_{2} \mathrm{OCOCH}_{3}(45)$}

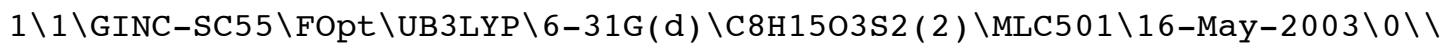
\# B3LYP/6-31G* OPT=(TIGHT, MAXCYC=100) FREQ MAXDISK $=134217728$ GEOM=CHEC K GUESS=READ \\rrad_z=opr_r=va-alb1cld3elf1-6dub3 -equal lowest at $h f \backslash \backslash$ $0,2 \backslash S, 0.1918860007,-1.46 \overline{6} 9449133,-0.5883643071 \backslash \mathrm{C},-1.0147456272,-0.3645$ $085308,0.0941175801 \backslash \mathrm{S},-2.2883850751,-1.0930242065,1.0740569962 \backslash \mathrm{C},-3.60$ $21577943,-1.4723203757,-0.1730092347 \backslash C, 1.4511854804,-0.2895875088,-1.2$ 
$001181955 \backslash 0,-1.2537443497,0.7680096745,-0.6321867661 \backslash \mathrm{C},-1.5950971687,1$ $.9760695955,0.1114357908 \backslash 0,2.2810356655,0.2459674797,-0.1657988121 \backslash \mathrm{C}, 3$ $.3780744146,-0.4937786355,0.1700051507 \backslash 0,3.6884310573,-1.5198665652,-0$ $.3884929176 \backslash \mathrm{C}, 4.1153839391,0.1462257974,1.3197715654 \backslash \mathrm{H}, 2.0548782551,-0$ $.8369448089,-1.9246770999 \backslash \mathrm{H}, 0.9448347964,0.5609663503,-1.6512075199 \backslash \mathrm{H}$, $-3.8263060411,-0.5845374519,-0.7678875925 \backslash \mathrm{H},-4.4917241291,-1.768627864$ $, 0.3894526569 \backslash \mathrm{H},-3.2897415018,-2.2915853553,-0.8244629025 \backslash \mathrm{H}, 3.51604298$ $54,0.0537051408,2.2324514999 \backslash \mathrm{H}, 4.2697692304,1.213211261,1.1341702108 \backslash \mathrm{H}$ , $5.0721471265,-0.3572882699,1.4621192819 \backslash \mathrm{C},-2.1931848312,2.9331525388$, $-0.9097650812 \backslash \mathrm{C},-0.3577588601,2.5278205484,0.8116518536 \backslash \mathrm{H},-2.351501897$ $3,1.7017676556,0.8561650094 \backslash \mathrm{H},-2.5050350407,3.8613081685,-0.4190225922$ $\backslash \mathrm{H},-1.4579404294,3.1829818394,-1.6828682386 \backslash \mathrm{H},-3.0666163236,2.48776040$ $61,-1.3962042973 \backslash \mathrm{H},-0.6213837839,3.4132234354,1.4012882065 \backslash \mathrm{H}, 0.0718813$ $196,1.7795731619,1.4845371157 \backslash \mathrm{H}, 0.4087043246,2.8126669604,0.0823546262$ $\backslash \backslash$ Version=DEC-AXP-OSF $/ 1-G 98 R e v A .11 .3 \backslash \mathrm{HF}=-1335.8912608 \backslash \mathrm{S} 2=0.754755 \backslash \mathrm{S} 2-1$ $=0 . \backslash \mathrm{S} 2 \mathrm{~A}=0.750017 \backslash \mathrm{RMSD}=8.868 \mathrm{e}-09 \backslash \mathrm{RMSF}=3.253 \mathrm{e}-06 \backslash \mathrm{Dipole}=-0.4984746,1.379$ $0475,-0.0238632 \backslash P G=C 01 \quad[X(C 8 H 1503 S 2)] \backslash \backslash @$

\section{$\mathrm{CH}_{3} \mathrm{SCH}\left(\mathrm{OCH}\left(\mathrm{CH}_{3}\right)_{2}\right) \mathrm{SCH}_{2} \mathrm{OCOCH}_{3}$}

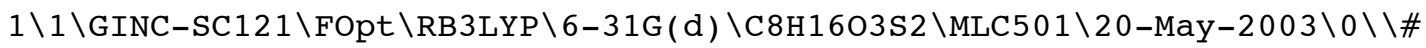
B3LYP/6-31G* OPT $=($ TIGHT, MAXCYC $=100)$ FREQ MAXDISK $=134217728 \backslash \backslash \mathrm{ralk} z=$ opr

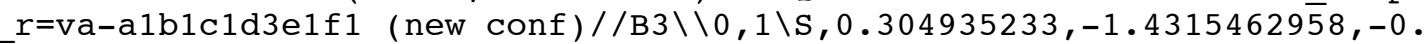
$\overline{5} 034474037 \backslash \mathrm{C},-0.8979964514,-0.3601850085,0.396162812 \backslash \mathrm{S},-2.2571232971,-$ $1.4081025825,1.0832923957 \backslash \mathrm{C},-3.0790138978,-1.9310985248,-0.4667534016 \backslash$ C, $1.4278540948,-0.155002289,-1.156892106 \backslash 0,-1.3426819062,0.6531334505$, $-0.4568093179 \backslash C,-1.7835004145,1.8657603195,0.2071378999 \backslash 0,2.2549860323$ $, 0.4432261155,-0.147896929 \backslash \mathrm{C}, 3.4682219329,-0.1428840257,0.0696550333 \backslash 0$ , $3.8880118087,-1.0757534728,-0.5738812096 \backslash \mathrm{C}, 4.1846597435,0.5342689995$, $1.212242362 \backslash \mathrm{H}, 2.0491866443,-0.6395849382,-1.9109812747 \backslash \mathrm{H}, 0.8373573992$, $0.6558931251,-1.5793361864 \backslash \mathrm{H},-3.1734204422,-1.0725343467,-1.1349044673$ $\backslash \mathrm{H},-4.0733910362,-2.2891893594,-0.1892215009 \backslash \mathrm{H},-2.5276430694,-2.731775$ $9595,-0.9634107613 \backslash \mathrm{H}, 3.6720721058,0.3034898329,2.1528908412 \backslash \mathrm{H}, 4.170711$ $8053,1.6210334036,1.08720515 \backslash \mathrm{H}, 5.2117305171,0.1711655685,1.2619922184 \backslash$ $\mathrm{C},-2.8233709369,2.5023507624,-0.7058897141 \backslash \mathrm{C},-0.5894471677,2.781786131$ $2,0.4741871977 \backslash \mathrm{H},-2.2612356472,1.5803267797,1.1539142777 \backslash \mathrm{H},-3.18948924$ $98,3.4397196846,-0.2729508089 \backslash \mathrm{H},-2.3862262974,2.7232356639,-1.68606693$ $1 \backslash \mathrm{H},-3.67500127,1.8308287962,-0.8505584876 \backslash \mathrm{H},-0.9041965501,3.662598014$ $7,1.0454691916 \backslash \mathrm{H}, 0.194667951,2.2711442459,1.0432299244 \backslash \mathrm{H},-0.1515154982$

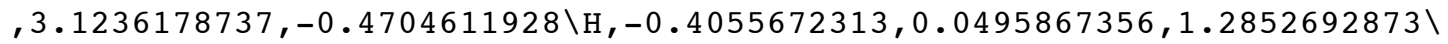
$\backslash$ Version=DEC-AXP-OSF $/ 1-G 03$ RevB . 02 $\backslash$ State $=1-A \backslash H F=-1336.5397739 \backslash \mathrm{RMSD}=4.82$ $2 e-09 \backslash \mathrm{RMSF}=1.786 \mathrm{e}-06 \backslash \mathrm{Dipole}=-0.4753544,1.4278607,0.1794523 \backslash \mathrm{PG}=\mathrm{C} 01] \mathrm{X}(\mathrm{C}$ $8 \mathrm{H} 1603 \mathrm{~S} 2$ ) $] \backslash \backslash @$

\section{$\mathrm{CH}_{3} \mathrm{SC} \cdot\left(\mathrm{OC}\left(\mathrm{CH}_{3}\right)_{3}\right) \mathrm{SCH}_{2} \mathrm{OCOCH}_{3}(46)$}

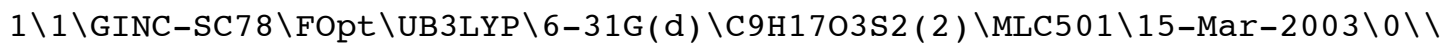
\#P B3LYP/6-31G(D) OPT=(TIGHT, MAXCYC=100) FREQ MAXDISK=65536000 GEOM=CH ECK GUESS=READ \\RAFT $(\mathrm{Z}=\mathrm{OBu} \mathrm{R}=\mathrm{VA})$ a $1 \mathrm{~b} 1 \mathrm{c} 2 \mathrm{~d} 1 \mathrm{e} 2 \mathrm{f} 1 / / \mathrm{B} 3 \backslash \backslash 0,2 \backslash \mathrm{S},-0.5673906256$ $, 0.4308539995,-1.3349892434 \backslash \mathrm{C}, 0.7665847077,0.506034444,-0.145627333 \backslash \mathrm{S}$, $1.0236130518,1.8898281637,0.9322732371 \backslash \mathrm{C}, 2.2584056702,2.8803146764,-0$. $0174898663 \backslash \mathrm{C},-1.9454967904,1.2064330722,-0.4289879762 \backslash 0,1.853705133,-0$ $.1767007112,-0.5923806837 \backslash \mathrm{C}, 2.2333687915,-1.4668747908,0.0282761879 \backslash 0$, $-2.5178512976,0.3559264799,0.5692138523 \backslash \mathrm{C},-3.5458822436,-0.4451741131$, $0.1662469867 \backslash 0,-4.0020480569,-0.4399865263,-0.9533569538 \backslash \mathrm{C},-4.01035075$ $36,-1.3174199144,1.3063751552 \backslash \mathrm{H},-1.579481341,2.0861479638,0.1002049158$ $\backslash \mathrm{H},-2.6944997053,1.4657949554,-1.1781365622 \backslash \mathrm{H}, 3.0962900981,2.241822053$ $3,-0.3030785665 \backslash \mathrm{H}, 2.6076079948,3.6728785198,0.6496631553 \backslash \mathrm{H}, 1.800115469$ $2,3.3207105252,-0.9062078455 \backslash \mathrm{H},-4.2091993547,-0.71191764,2.1957862013 \backslash$ 
$\mathrm{H},-3.2220864478,-2.0324684527,1.5664029623 \backslash \mathrm{H},-4.9090695222,-1.85756094$ $49,1.0070343661 \backslash \mathrm{C}, 3.407846852,-1.9237215289,-0.8365316299 \backslash \mathrm{C}, 1.06463975$ $69,-2.4506853395,-0.0545999661 \backslash \mathrm{C}, 2.6669295323,-1.233772834,1.477457862$ $8 \backslash \mathrm{H}, 3.7949236636,-2.8827720672,-0.4762797097 \backslash \mathrm{H}, 3.0933986334,-2.0434018$ $074,-1.8780727013 \backslash \mathrm{H}, 4.2180489488,-1.1881315058,-0.8032186405 \backslash \mathrm{H}, 1.36690$ $22752,-3.4205220985,0.356512973 \backslash \mathrm{H}, 0.2052889841,-2.0894610028,0.5187737$ $557 \backslash \mathrm{H}, 0.748695555,-2.5976149628,-1.0919666456 \backslash \mathrm{H}, 3.0309869646,-2.171769$ $2972,1.9124119769 \backslash \mathrm{H}, 3.471611884,-0.4936155155,1.5269419632 \backslash \mathrm{H}, 1.8341877$ $158,-0.8737493044,2.0881582579 \backslash \backslash$ Version=DEC-AXP-OSF $/ 1-G 98 \mathrm{RevA} .11 .3 \backslash \mathrm{HF}=$ $-1375.2020858 \backslash \mathrm{S} 2=0.754755 \backslash \mathrm{S} 2-1=0 . \backslash \mathrm{S} 2 \mathrm{~A}=0.750017 \backslash \mathrm{RMSD}=6.605 \mathrm{e}-09 \backslash \mathrm{RMSF}=8.1$ $27 e-06 \backslash \mathrm{Dipole}=0.6297237,-0.1223274,0.7480766 \backslash \mathrm{PG}=\mathrm{C} 01[\mathrm{X}(\mathrm{C} 9 \mathrm{H} 1703 \mathrm{~S} 2)] \backslash \backslash @$

\section{$\mathrm{CH}_{3} \mathrm{SCH}\left(\mathrm{OC}\left(\mathrm{CH}_{3}\right)_{3}\right) \mathrm{SCH}_{2} \mathrm{OCOCH}$}

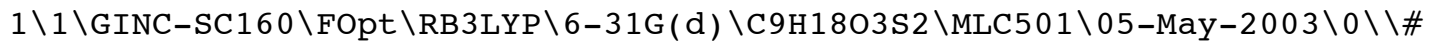
B3LYP $/ 6-31 \mathrm{G} *$ OPT $=($ MAXCYC $=100$, TIGHT $)$ FREQ MAXDISK $=131072000$ GEOM=CHECK

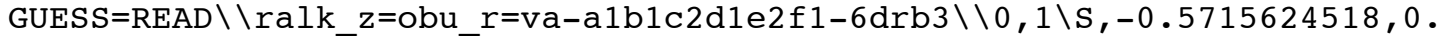
$5867355188,-1.19 \overline{0} 38346 \overline{7} 3 \backslash \mathrm{C}, 0.7084168857,0.4074426679,0.1552698474 \backslash \mathrm{S}, 1$. $1336323817,1.9698698194,1.0409860567 \backslash \mathrm{C}, 1.9619302671,2.8789214547,-0.31$ $0080351 \backslash C,-1.9810546718,1.2019201332,-0.2216753441 \backslash 0,1.8154697973,-0.1$ $51579444,-0.478760435 \backslash \mathrm{C}, 2.3066011678,-1.4557969883,-0.0358983815 \backslash 0,-2$. $5712344086,0.1990808869,0.6216360972 \backslash \mathrm{C},-3.6039102691,-0.5137332174,0.0$ $879617337 \backslash 0,-4.0607744288,-0.3151633573,-1.0134298273 \backslash \mathrm{C},-4.0798918279$, $-1.5618951431,1.064393305 \backslash \mathrm{H},-1.6458662551,1.99283685,0.451026309 \backslash \mathrm{H},-2$. $7194603209,1.5711320848,-0.934474866 \backslash \mathrm{H}, 2.8124691504,2.3032610722,-0.67$ $97569156 \backslash \mathrm{H}, 2.3113508736,3.8231967786,0.1144211839 \backslash \mathrm{H}, 1.2637776312,3.079$ $5991637,-1.1263030811 \backslash \mathrm{H},-4.3012424998,-1.1072303244,2.0349620694 \backslash \mathrm{H},-3$. $2911867868,-2.3055312213,1.2228652693 \backslash \mathrm{H},-4.96905211,-2.0513864635,0.66$ $59139889 \backslash \mathrm{C}, 3.5119157391,-1.6914485167,-0.9490699578 \backslash \mathrm{C}, 1.2392817828,-2$. $5340007742,-0.2649318876 \backslash \mathrm{C}, 2.7458186125,-1.3962697738,1.4331379404 \backslash \mathrm{H}, 3$ $.9758354315,-2.6588586259,-0.7295204333 \backslash \mathrm{H}, 3.201451766,-1.683233414,-1$. $9985360202 \backslash \mathrm{H}, 4.2601760742,-0.9055600597,-0.8049088751 \backslash \mathrm{H}, 1.6401513387,-$ $3.5187799638,-0.0000057661 \backslash \mathrm{H}, 0.3470640318,-2.3666210942,0.348190815 \backslash \mathrm{H}$, $0.9312564763,-2.5538248957,-1.3145617226 \backslash \mathrm{H}, 3.2136657434,-2.3443213612$, $1.7211790764 \backslash \mathrm{H}, 3.4683371799,-0.589148558,1.5858040393 \backslash \mathrm{H}, 1.9009614213$, $1.2287440104,2.1096700002 \backslash \mathrm{H}, 0.2848581803,-0.2320151066,0.934185392 \backslash \backslash \mathrm{Ve}$ rsion=DEC-AXP-OSF $/ 1-G 98 R e v A .11 .3 \backslash \mathrm{HF}=-1375.8518196 \backslash \mathrm{RMSD}=8.287 \mathrm{e}-09 \backslash \mathrm{RMSF}=$ $5.301 e-06 \backslash \mathrm{Dipole}=0.6144762,-0.497542,0.816135 \backslash \mathrm{PG}=\mathrm{C} 01[\mathrm{X}(\mathrm{C} 9 \mathrm{H} 1803 \mathrm{~S} 2)] \backslash \backslash \mathrm{a}$ 